\title{
Wide Piezoelectric Tuning of LTCC Bandpass Filters
}

\author{
Mahmoud Al Ahmad
}




\title{
Wide Piezoelectric Tuning of LTCC Bandpass Filters
}

\author{
Mahmoud Al Ahmad
}

Vollständiger Abdruck der von der Fakultät für Elektrotechnik und Informationstechnik der Technischen Universität München zur Erlangung des akademischen Grades eines

\section{Doktor-Ingenieurs}

genehmigten Dissertation.

Vorsitzender:

Univ.-Prof. Dr.-Ing. Wolfgang Utschick

Prüfer der Dissertation:

1. Univ.-Prof. Dr. techn. Peter Russer

2. Univ.-Prof. Dr.-Ing. habil. Robert Weigel

Friedrich-Alexander-Universität Erlangen-Nürnberg

Die Dissertation wurde am 14.09.2005 bei der Technischen Universität München eingereicht und durch die Fakultät für Elektrotechnik und Informationstechnik am 18.04.2006 angenommen. 


\section{Abstract}

This work treats design and fabrication issues associated with innovative tunable front-end components which combine two different ceramic technologies, namely multilayer ceramic circuit boards (low temperature cofired ceramics or LTCC) and piezoelectric actuator technology within a single device. The need for such components is particularly arising due to the increasing number of wireless services and associated frequency bands in the range between 0.5 and $2.5 \mathrm{GHz}$. This has led in the past to the concept of software-defined radio (SDR) which would provide a cost-efficient solution by treating signals digitally and software-controlled up to the highest possible frequencies and as close as possible towards the transmit antenna, while the final analogue section at the antenna comprises only few high-performing and frequencyagile, tunable components. However, as a consequence of demanding component specifications, SDR has not yet found noticeable application in consumer markets despite ongoing search for suitable device concepts and fabrication technologies.

Similar to the known micro-electromechanical (MEMS) approaches for tuning and switching, this work presents a modified parallel plate capacitor with high-permittivity dielectric and piezoelectrically movable top electrode as a tuning element. Like in MEMS solutions, there is no tunable dielectric material required for tuning as for example paraelectric material, which would introduce additional losses. The proposed device therefore has the potential for a high quality factor. Contrasting MEMS, piezoelectric actuators exhibit proven reliability and lifetime. Also sticking of contact surfaces can be overcome by the actuator force. The size of the actuator in the order of several millimeters is not impedimental in the present context, since it compares well to the size of planar integrated filters in the frequency range mentioned.

The vertical movement of the electrode opens an air gap above the dielectric film which allows for substantial lowering of the effective dielectric constant and capacitance. When applied as a shunt capacitor in a coupled microstrip lines LTCC bandpass filter, the center frequency of the filter is tuned from $1.1 \mathrm{GHz}$ to $2.6 \mathrm{GHz}$ (tunability of $135 \%$ ) with $200 \mathrm{~V}$ control voltage and low insertion loss of value $4 \mathrm{~dB}$ (at zero-bias) to $2 \mathrm{~dB}$ (at the maximum bias). For a more compact size, one electrode of the piezoelectric element is simultaneously used as the center microstrip line of a filter employing three coupled lines. Its equivalent circuit has been used to explore the change of the capacitor parameters across the entire tuning range. The capacitor varied from $7 \mathrm{pF}$ to $1.35 \mathrm{pF}$ with a quality factor between 60 and 160. The quality factor could be improved by a factor of 7 when the metallization of the piezoelectric actuator, changes from $80 \mathrm{~nm}$ to $500 \mathrm{~nm}$.

This thesis discusses also the effects of tuning mechanism on the overall quality factor, return loss, insertion loss, and the relative bandwidth at the mid of the band as a function of frequency across the entire tuning range. The analysis of the device by full-wave simulation reveals a high potential tuning range from $0.8 \mathrm{GHz}$ to $2.8 \mathrm{GHz}$ when the thin-film processability of the LTCC surface is properly controlled. The feasibility of tuning using piezoceramic varactors is explored. A systematic approach for designing wideband tunable combline microstrip filters is presented. The assembly and interconnect technology between LTCC microstrip structures 
and piezoceramic element is important for the device performance. Control over the thin film air-gap capacitor on the thick film LTCC substrate requires the integration of a polishing step into the processing sequence. Switching speed, dynamic behavior as well as power consumption are being addressed. 


\section{Acknowledgments}

First of all, I would like to express my gratitude and thanks to Professor Peter Russer for his constant support, encouragement, and inspiration during my dissertation. I am also grateful to a lot of people and the fact that I could get the chance to work with the people at the CT MM2. Much thanks to Dr. Richard Matz, please receive my thanks for all help and patience, which cannot be overestimated (it should never ever be forgotten). I am very grateful for help from Ruth Maenner during the fabrication process. I wish to acknowledge experienced support in polishing technology by Mr. Eberhard Hoyer and fruitful discussions on piezoceramic device performance with Andreas Wolff. Argillon GmbH contributed useful advice and application-

specific modifications of commercial piezoceramic elements. I wish to express my gratitude to Dr. W. Rossner at Siemens Corporate Technology, for his encouragement and support. My thanks go to all my colleagues at CT MM2 for their support, collaboration, encouragement, and friendship during these years, special thanks to Dr. Steffen Walter, Dr. Wilhelm Metzger and Dr. Ashkan Naeini.

Mahmoud Al Ahmad

Munich September $13^{\text {th }} 2005$ 


\title{
List of Abbreviations
}

\author{
ADC Analogue to Digital Converter \\ ADS Advance Design System \\ AFE Analogue Front End \\ ASM Antenna Switch Module \\ bp bandpass \\ bs bandstop \\ BST Barium Strontium Titanate \\ DAC Digital to Analog Converter \\ dB decible \\ DC Direct Current \\ DFE Digital Front End \\ EE Even-Even mode \\ EM ElectroMagnetic \\ FBW Fractional BandWidth \\ FDM Finite Difference Method \\ FE Front End \\ FEM Finite Element Method \\ GaAs Gallium Arsenide \\ High-K Insulating dielectric material with very high dielectric constant \\ hp highpass \\ IF Intermediate Frequency \\ LHS left-hand of the $s$-plane \\ LTCC Low Temperature Cofired Ceramic \\ lp lowpass \\ lpp lowpass prototype \\ MEMS Micro-ElectroMechanical System \\ MoM Moment of Method \\ OE Odd-Even mode \\ OO Odd-Odd mode \\ PET Piezoelectric Transducer \\ PTF Piezoelectric Tunable Filter \\ PSD Position Sensitive Detector \\ PZT lead Zirconate Titanate \\ RHS right-hand of the $s$-plane \\ RF Radio Frequency \\ Rx Reception band \\ SDR Software Defined Radio \\ SMT Surface Mount Technology \\ SRF Self-Resonance Frequency \\ TEM Transverse ElectroMagnetic \\ TLM Transmission Line Matrix \\ Tx Transmission band \\ VCO Voltage Controlled Oscillator
}




\section{List of Symbols}

$Q \quad$ quality factor of a varactor.

$I L$ insertion loss of the filter defined at specific frequency.

$P_{L} \quad$ power transmitted to the load

$P_{\text {in }} \quad$ incident power

$P_{A} \quad$ power absorbed by the filter

$P_{R} \quad$ power reflected back to the generator

$g_{i} \quad i^{t h}$ element value of the prototype low pass filter

$\epsilon_{0} \quad$ vacuum permittivity of $8.85 \times 10^{-12}(\mathrm{pF} / \mathrm{m})$

$\epsilon_{r e}$ effective permittivity of the air plus the high-K dielectric layer

$A \quad$ area of the capacitor

$d_{\text {air }}$ effective air gap inside the capacitor at the tip of the cantilever

$d_{K} \quad$ thickness of the high-K dielectric layer

$\epsilon_{K} \quad$ effective permittivity of the high-K dielectric layer.

$d_{31} \quad$ piezoelectric constant of the material of $230 \mathrm{pm} / \mathrm{V}$

$V \quad$ actuation applied voltage

$L \quad$ length of the piezoelectric cantilever of $7 \mathrm{~mm}$

$T \quad$ thickness the piezoelectric cantilever of $0.13 \mathrm{~mm}$

$f \quad$ resonance frequency of the transmission line

$Z_{\text {res }} \quad$ impedance of the transmission line

$\theta$

$C_{\max }$

$C_{\text {min }}$

electrical length of the line at resonance

largest capacitance

$f_{\max }$ highest tunable frequency

$f_{\min } \quad$ lowest tunable frequency

$\lambda \quad$ guided wavelength in the medium of the propagation

$\lambda_{0} \quad$ free-space wavelength

$\epsilon_{r} \quad$ effective relative dielectric constant

V Voltage vector

I Current vector

$\underline{V}_{i} \quad$ Voltage value at node $i$

$\underline{I}_{i} \quad$ Current value at node $i$

$\mathbf{z}$ network impedance normalized matrix

$z_{i j} \quad$ Normalized impedance between ports $i$ and $j$

$Z_{i j} \quad$ Non-normalized impedance between ports $i$ and $j$

$\mathbf{S} \quad$ Scattering matrix

$S_{i i} \quad$ reflection coefficients at port $i$

$S_{i j} \quad$ transmission coefficients between ports $i$ and $j$

b normalized reflected voltage wave vector

a normalized incident voltage wave vector

$\underline{a}_{i} \quad$ normalized incident voltage wave at port $i$

$\underline{b}_{i} \quad$ normalized reflected voltage wave at port $i$

$Z_{i n} \quad$ terminating line impedance

$Y_{\text {in }} \quad$ terminating line admittance

$\theta_{0} \quad$ midband electrical length of the lines

$\Delta \omega \quad$ passband bandwidth

$\dagger \quad$ Hermitian conjugate

* complex conjugate 


\begin{tabular}{|c|c|}
\hline $\mathbf{U}$ & unitary matrix \\
\hline$A_{p}$ & minimum passband gain \\
\hline$A_{s}$ & maximum stopband gain \\
\hline$\omega_{0}$ & normalized center frequency \\
\hline$\omega_{p u}$ & upper passband normalized center frequency \\
\hline$\omega_{s u}$ & upper stopband normalized center frequency \\
\hline$\omega_{p l}$ & lower passband normalized center frequency \\
\hline$\omega_{s l}$ & lower stopband normalized center frequency \\
\hline$p$ & the normalized complex frequency variable \\
\hline$\sigma$ & real part of the normalized complex frequency \\
\hline$\omega$ & imaginary part of the normalized complex frequency \\
\hline$S(p)$ & normalized reflection coefficient in terms of the normalized complex frequency variable \\
\hline$Z(p)$ & normalized network impedance in terms of the normalized complex frequency variable \\
\hline$Z_{\text {in }}(p)$ & normalized input impedance in terms of the normalized complex frequency variable \\
\hline$Y(p)$ & normalized admittance network in terms of the normalized complex frequency variable \\
\hline$Y_{\text {in }}(p)$ & normalized input admittance in terms of the normalized complex frequency variable \\
\hline$\Re\{p\}$ & real part of $p$ \\
\hline$\Im\{p\}$ & imaginary part of $p$ \\
\hline$N(p)$ & normalized numerator of the rational function of the normalized $S(p)$ \\
\hline$D(p)$ & normalized denominator of the rational function of the normalized $S(p)$ \\
\hline$A^{2}\left(\omega^{2}\right)$ & amplitude response function \\
\hline$B\left(\omega^{2}\right)$ & the numerator of the rational function of the amplitude response function \\
\hline$C\left(\omega^{2}\right)$ & the denominator of the rational function of the amplitude response function \\
\hline$\left|S_{11}(\omega)\right|$ & magnitude value of $S_{11}(\omega)$ \\
\hline$\left|S_{12}(\omega)\right|$ & magnitude value of $S_{12}(\omega)$ \\
\hline$Z_{i}$ & the $i^{\text {th }}$ element of the ladder circuit prototype \\
\hline$C$ & capacitance \\
\hline$C_{i}, C_{s}$ & $i^{t h}$ self-capacitance \\
\hline$C_{i, i+1}, C_{m}$ & $i^{\text {th }}$ mutual capacitance \\
\hline$\theta_{\min }$ & minimum midband electrical length \\
\hline$C_{t}$ & total capacitance \\
\hline$S / H$ & gap ratio in a coupled line \\
\hline$W / H$ & shape ratio in a coupled line \\
\hline$x$ & distance from the clamping point \\
\hline$t$ & thickness of a single piezoelectric layer \\
\hline$L \times W \times T$ & cantilever dimensions \\
\hline$h$ & distance between the laser source and the PSD detector \\
\hline$d$ & reference distance that result from the calibration \\
\hline$D$ & cantilever deflection \\
\hline$\tau_{F}$ & tunability factor \\
\hline$f_{0}$ & center frequency of the frequency agile component at no bias \\
\hline$f_{V_{\max }}$ & center frequency of the component at the maximum applied bias \\
\hline$S_{21}$ & transmission coefficient of a filter \\
\hline$S_{11}$ & reflection coefficient of a filter \\
\hline Qloaded & loaded quality factor \\
\hline$S_{21}\left(f_{0}\right)$ & transmission coefficient of the filter at the mid-band frequency $f_{0}$ \\
\hline Qunloaded & unloaded quality factor \\
\hline$K_{n m}$ & mutual-coupling between lines $\mathrm{n} \& \mathrm{~m}$ \\
\hline$L_{i}$ & inductance of the transmission line (i) \\
\hline$R_{a}$ & filter ohms and dielectric losses \\
\hline $\begin{array}{l}R_{s} \\
\partial f\end{array}$ & cantilever ohmic losses \\
\hline $\begin{array}{l}\frac{\partial f}{\partial V} \\
\partial C\end{array}$ & tuning sensitivity versus the bias voltage \\
\hline$\frac{\partial V}{\partial V}$ & change in the capacitance in terms of voltage change \\
\hline
\end{tabular}


$V_{\max }$ maximum operation voltage

$V_{t} \quad$ tuned operation voltage

$E \quad$ electrostatic energy

$\Delta E \quad$ maximal change in electrostatic energy per tuning step

$N \quad$ filter order which represents the poles number

$R \quad$ Resistor

$L \quad$ Inductor

C Capcitor

$L_{A} \quad$ insertion loss

$L_{A} \quad$ return loss

$P_{L R} \quad$ power loss ratio

$\xi \quad$ ripple level

$s \quad$ laplace transformation

C static capacitances matrix

$\eta \quad$ characteristic admittance matrix

$\nu \quad$ phase velocity

$\beta \quad$ wave number

$Y_{i j} \quad$ admittance between ports $i$ and $j$

$C_{i j} \quad$ coupling capacitance between ports $i$ and $j$ 



\section{List of Figures}

1.1. SDR system architecture (After Lucent Technologies) . . . . . . . . . . . . . 4

2.1. Frequency response, theoretical bandpass filter. . . . . . . . . . . . . . . 7

2.2. The ladder network. . . . . . . . . . . . . . . . . . . . . . 12

2.3. The complete synthesis process . . . . . . . . . . . . . . . . . . . . 14

2.4. General filter network configuration. . . . . . . . . . . . . . . . . 15

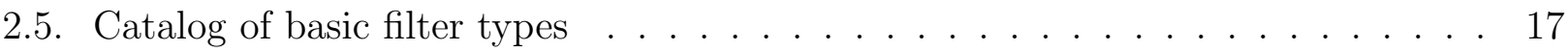

2.6. Poles of the second order Butterworth transfer function. . . . . . . . . . . . . 20

2.7. Poles of the second order Butterworth. . . . . . . . . . . . . . . . . . . . . 20

2.8. Realization techniques. . . . . . . . . . . . . . . . . . . 21

2.9. Butterworth lowpass characteristic. . . . . . . . . . . . . . . . . 22

2.10. Two-element lowpass prototype. . . . . . . . . . . . . . . . . . 22

2.11. Ladder circuit for lowpass filter prototype of three order and their element definitions. . . . . . . . . . . . . . . . . . 24

2.12. Chebyshev polynomials curves. . . . . . . . . . . . . . . . . 25

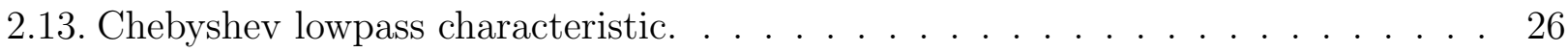

2.14. Poles of the second order Chebyshev transfer function . . . . . . . . . . . . . . 28

2.15. Poles of the $6^{\text {th }}$ order Chebyshev imposed with Butterworth of the same order. . 29

2.16. Transformed poles of the low prototype to $\omega_{c}$. . . . . . . . . . . . . . . 32

2.17. Transformed poles of the low prototype to $\omega_{c}=1.5, \omega<\omega_{c}=0.5, \omega>\omega_{c}=$

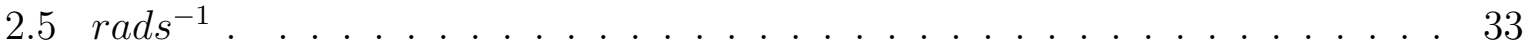

2.18. Bandpass filter circuit resulted from the transformation of the lowpass prototype. 34

2.19. Catalog of bandpass filter types . . . . . . . . . . . . . . . . . . 34

2.20. Amplitude response for the three bandpass filter types. . . . . . . . . . . . . . 35

2.21. Bandpass filter poles pattern. . . . . . . . . . . . . . . . . . 37

2.22. The transformed poles pattern from lowpass to bandpass filter at $\omega_{0}=1.75$ and

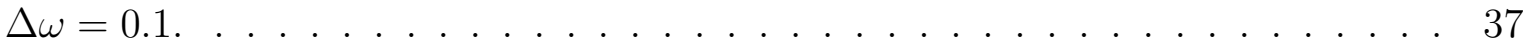

2.23. A symmetrical coupled-line N-port. . . . . . . . . . . . . . . . . . . . 38

2.24. Mutual and self-capacitances of periodic array of interdigitated microstrip conductors. . . . . . . . . . . . . . . . . . . . 4 40

2.25. General structure of a microstrip combline bandpass filter. . . . . . . . . . . . . 41

2.26. Tunability, tunable frequency response. . . . . . . . . . . . . . . . . . . 42

2.27. Varactor loaded resonator. . . . . . . . . . . . . . . . . . . . . 43

2.28. Combline filter characteristic . . . . . . . . . . . . . . . . . . . . 45 
2.29. Equivalent circuit of stubs representations . . . . . . . . . . . . . . . 46

2.30. The corresponding lowpass prototype filter of a ladder network. . . . . . . . . . 47

2.31. Formulation of inverters in the combline filter. . . . . . . . . . . . . . . . . 48

2.32. Combline Filter equivalent circuit. . . . . . . . . . . . . . . . . . . . . . 48

2.33. Input-output network . . . . . . . . . . . . . . . . . . . . . 50

2.34. Real part of the input admittance as a function of the electrical length. . . . . . 52

2.35. Input port of the filter after scaling. . . . . . . . . . . . . . . . . . 52

2.36. Coupling network between internal nodes of the filter. . . . . . . . . . . . . 54

2.37. The passband bandwidth as a function of the operating frequency. . . . . . . . . 56

2.38. Normalized instantaneous bandwidth. . . . . . . . . . . . . . . . . . . 57

2.39. Chebyshev ripple over tuning. . . . . . . . . . . . . . . . . . . . . . . . . 61

2.40. Example: filter equivalent circuit. . . . . . . . . . . . . . . . . 65

2.41. First order computed tuned filter performance, $\Delta \omega_{1}=\theta_{1} / a=168 \mathrm{MHz}$, thus, $a$ $=1.17$, hence, $\Delta \omega_{0}=382 \mathrm{MHz}$ and $\Delta \omega_{2}=719 \mathrm{MHz} \ldots \ldots$. . . . . . . . . 66

2.42. self and mutual static capacitance definitions for the first order filter. . . . . . . 66

2.43. Example: second-order filter equivalent circuit. . . . . . . . . . . . . . . . . . . 67

2.44. Second order computed tuned filter performance, $\Delta \omega_{1}=\theta_{1} / a=171 \mathrm{MHz}$, thus, $\Delta \omega_{0}=394 \mathrm{MHz}$ and $\Delta \omega_{2}=744 \mathrm{MHz} \ldots \ldots \ldots \ldots \ldots$. . . . . . . 69

2.45. self and mutual static capacitance definitions for the second order filter. . . . . . 69

2.46. Capacitances deviation, the numbers represent the difference $\Delta C$ between the two capacitors $C_{1}$ and $C_{2}$. . . . . . . . . . . . . . . . . . . 70

3.1. Piezoelectric bimorph cantilever with dimension $7 \mathrm{~mm} \times 1 \mathrm{~mm} \times 0.48 \mathrm{~mm}$. . . . . 72

3.2. Piezoelectric bimorph cantilever cross section. . . . . . . . . . . . . . . . 72

3.3. LTCC process flow chart, Siemens CT MM2 Application Notes. . . . . . . . . . 73

3.4. Structure used to fix the cantilever to the LTCC substrate during the assembly process. . . . . . . . . . . . . . . . . . . . 74

3.5. Assembly process: glue. . . . . . . . . . . . . . . . . . . . . 75

3.6. Experiment Setup. . . . . . . . . . . . . . . . . . . . 75

3.7. Triangulation calculation. . . . . . . . . . . . . . . . . 76

3.8. Characterization of piezoelectric actuator glued to LTCC substrate . . . . . . . 77

4.1. Schematic of the piezoelectric LTCC varactor. . . . . . . . . . . . . . . . . . . 79

4.2. Tuning of a multilayer capacitor by adjusting the width of the air gap. . . . . . 81

4.3. Typical cantilever deflection of a commercially available piezoelectric actuator. . 81

4.4. One-pole microstrip combline bandpass filter. . . . . . . . . . . . . . . . . . 82

4.5. Schematic of the tunable piezoelectric LTCC combline filter. . . . . . . . . . . 83

4.6. Designed filter cross section, $w$ is the width of the input/out put lines, $T$ is the metallization thickness, $H$ is the thickness of the substrate, $\epsilon$ is the dielectric constant of the substrate, $S$ is the spacing between the lines, and $w_{m}$ is the width of the internal line, it is predefined by the width of the cantilever . . . . . 84

4.7. Simulation model . . . . . . . . . . . . . . . . . . . . . . . . 85

4.8. Simulated tuning of the bandpass filter by the variable capacitor. . . . . . . . . 86

4.9. Fabrication process. . . . . . . . . . . . . . . . . 87 
4.10. The fabricated tunable bandpass filter. . . . . . . . . . . . . . . . . . 88

4.11. RF measurements setup. . . . . . . . . . . . . . . . . . . . . . 89

4.12. Set of measured insertion loss and return loss responses for various control voltages. 90

4.13. Tuned bandpass filter with variable capacitor. . . . . . . . . . . . . . . . . 91

4.14. Measured frequency and the calculated corresponding air gap versus applied DC bias. . . . . . . . . . . . . . . . . . . . . . . . . . . . . . .

4.15. The return loss as a function of the tuned mid-band frequency. . . . . . . . . . . 92

4.16. The insertion loss and the unloaded quality factor as a function of the tuned mid-band frequency. . . . . . . . . . . . . . . . . . . . 92

4.17. Measured mid-band center frequency and the relative bandwidth as a function of the control bias voltage. . . . . . . . . . . . . . . . . . . . . 93

4.18. Equivalent circuit of the first order. . . . . . . . . . . . . . . . . . . . . 94

4.19. The performance of the equivalent circuit of the first order . . . . . . . . . . . . 95

4.20. Measured capacitance and the calculated corresponding air gap versus applied DC bias. . . . . . . . . . . . . . . . . . . . . . 96

4.21. Measured tunable coupling as a function of the deflection. . . . . . . . . . . . 96

4.22. Effective permittivity and the corresponding air gap versus actuation voltage. . 97

4.23. Capacitance density versus actuation voltage. . . . . . . . . . . . . . 97

4.24. Measured tunable frequency and the quality factor versus capacitance variation. 98

4.25. Layout of the 2 -pole tunable filter. . . . . . . . . . . . . . . . . . . . . . . 99

4.26. Simplified fabrication process for the second order. . . . . . . . . . . . . . 100

4.27. The fabricated second order filter. . . . . . . . . . . . . . . . . . . 101

4.28. Measured tuning curves for the second order filter. . . . . . . . . . . . . . . . . 102

4.29. Measurements and simulations of the second order. . . . . . . . . . . . . . . . 102

4.30. The most lower tuning range could be achieved within this sample. . . . . . . . 103

4.31. The most upper tuning range could be achieved within this sample. . . . . . . . 103

4.32. The tuned frequency for the input $\left(S_{11}\right)$ and the output $\left(S_{22}\right)$ reflection loss versus the actuation voltage. . . . . . . . . . . . . . . . 104

4.33. Comparison: measured attenuation for the first and the second orders. . . . . . 104

4.34. Equivalent circuit of the second order filter. . . . . . . . . . . . . . . . . . . . . . . . . . 105

4.35. Equivalent circuit and measurements. . . . . . . . . . . . . . . . 106

4.36. Tuned capacitance and quality factor versus variable air gap of the second order filter. . . . . . . . . . . . . . . . . . . . 107

4.37. Tuned mutual coupling versus variable air gap of the second order filter. . . . . 107

5.1. Device cross-section. . . . . . . . . . . . . . . . . . . . . 112

5.2. Hysteresis in capacitance as a function of the applied voltage. . . . . . . . . . 113

5.3. Tuning sensitivity in frequency versus control voltage. . . . . . . . . . . . . 115

6.1. Schematic of the piezoelectric tuned multi capacitors. . . . . . . . . . . . . 117

6.2. 3D-view of the proposed-configuration. . . . . . . . . . . . . 118

6.3. Device outlook circuit. . . . . . . . . . . . . . . . . . . . . 118

6.4. Multi-tuned capacitors with single cantilever. . . . . . . . . . . . . . . 119

6.5. Outlook: device performance. . . . . . . . . . . . . . . . 119 
List of Figures

6.6. Schematic of the fixing procedure using mask. . . . . . . . . . . . . . . . 120

A.1. 2-port network parameters definition. . . . . . . . . . . . . . . . 127 


\section{List of Tables}

2.1. Element values for the Butterworth Ladder Filter with $g_{0}=1, \omega_{c}=1$, and $\mathrm{n}=$

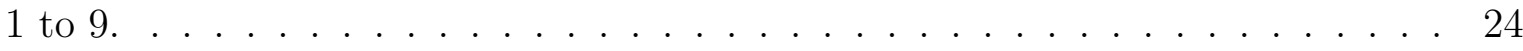

2.2. Chebyshev first kind polynomials . . . . . . . . . . . . . . 25

2.3. Element values for the Chebyshev Ladder Filter with $0.01 \mathrm{~dB}$ ripple with $g_{0}=$

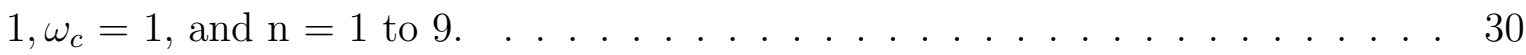

2.4. Element values for the Chebyshev Ladder Filter with $0.1 \mathrm{~dB}$ ripple with $g_{0}=1, \omega_{c}$

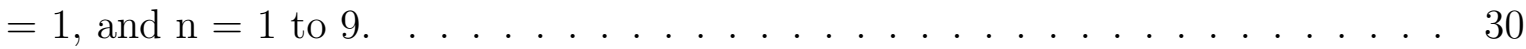

2.5. Elements values for a bandpass filter for various types. . . . . . . . . . . 35

4.1. Filter physical dimensions . . . . . . . . . . . . . . . . . 84

4.2. Dielectric layers. . . . . . . . . . . . . . . . . . . 86

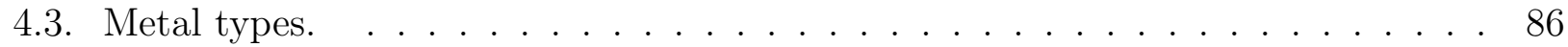

4.4. Equivalent circuit fixed parameters of the first order . . . . . . . . . . . . 94

4.5. Filter physical dimensions of the second order . . . . . . . . . . . . . . . 99

4.6. Equivalent circuit fixed parameters of the second order filter . . . . . . . . . 105

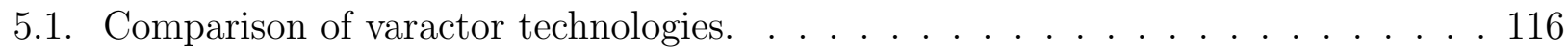





\section{Contents}

1. Introduction 1

1.1. State of The Art . . . . . . . . . . . . . . . . 1

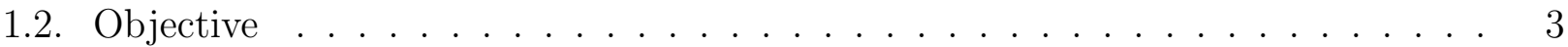

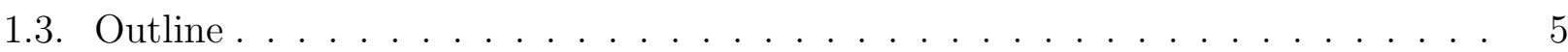

2. Fundamentals of Tunable Filter Theory $\quad 7$

2.1. Network Synthesis Method . . . . . . . . . . . . . . . . . . . . 8

2.1.1. Synthesis Techniques . . . . . . . . . . . . . . . 8

2.1.2. Ladder Network . . . . . . . . . . . . . . . . . . . . . . . . . . . 12

2.1.3. Losses of Two-Port Network . . . . . . . . . . . . . . . . . . . 15

2.2. Lowpass Prototype Filter . . . . . . . . . . . . . . . . . 16

2.2.1. Butterworth Filters . . . . . . . . . . . . . . . . . . 16

2.2.2. The Chebyshev Prototype . . . . . . . . . . . . . . . . 24

2.2.3. Bessel Filters . . . . . . . . . . . . . . . . . . . . . 31

2.2.4. Impedance scaling and Frequency Transformation . . . . . . . . . . . . 31

2.2.5. Poles Pattern of a Lowpass Filter . . . . . . . . . . . . . . . . . 31

2.3. Bandpass Filter Design . . . . . . . . . . . . . . . . . . 32

2.3.1. Poles Pattern in the Bandpass filter . . . . . . . . . . . . . . . . 35

2.3.2. Filters with Distributed Elements . . . . . . . . . . . . . 38

2.3.3. The Combline Filters . . . . . . . . . . . . . . . . . . . 40

2.3.4. Tunability . . . . . . . . . . . . . . . . . . . . 41

2.3.5. Center Frequency of a Varactor Loaded Resonator . . . . . . . . . . . . . 42

2.4. Tunable Combline Filter . . . . . . . . . . . . . . . . . . . . . 45

2.4.1. Filter Equivalent Circuit . . . . . . . . . . . . . . . . . 46

2.4.2. Narrowband Filter Design . . . . . . . . . . . . . . . . . . 54

2.4.3. Filter Parameters Selection . . . . . . . . . . . . . . . . . . . 55

2.5. Example . . . . . . . . . . . . . . . . . . . . . . 62

2.5.1. First-Order Filter . . . . . . . . . . . . . . . . . . . . . . . 62

2.5.2. Second-Order Filter . . . . . . . . . . . . . . . . 67

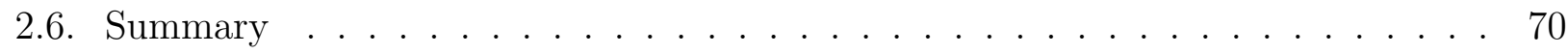


$\begin{array}{ll}\text { 3. Technology } & 71\end{array}$

3.1. Piezoelectric Bimorph Cantilever . . . . . . . . . . . . . . . . 71

3.2. Standard LTCC Process . . . . . . . . . . . . . . . . . . . . . . 73

3.3. The Assembly Process . . . . . . . . . . . . . . . . . . . . . . . . . . . 74

3.4. PZT Cantilever Characterization . . . . . . . . . . . . . . . . . . . 75

3.5. Measurements and Analysis . . . . . . . . . . . . . . . . . . 77

4. Design of Tunable LTCC Bandpass Filters $\quad 79$

4.1. Principle of Operation . . . . . . . . . . . . . . . . . 79

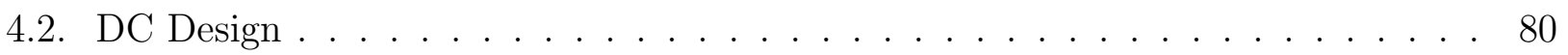

4.3. Tunable Bandpass Filter . . . . . . . . . . . . . . . . . . . . . . 82

4.4. Filter Synthesis Design . . . . . . . . . . . . . . . . . . . 83

4.5. Device Simulation . . . . . . . . . . . . . . . . . . . . . 85

4.5.1. Simulation Tools . . . . . . . . . . . . . . . . . . 85

4.6. Device-Specific Fabrication Process . . . . . . . . . . . . . . . . . . . 87

4.6.1. The Fabricated Device . . . . . . . . . . . . . . . . . . . 88

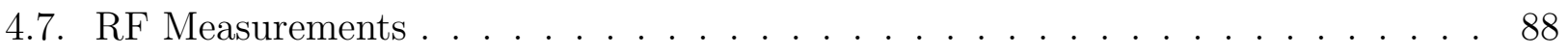

4.7.1. Measurements Setup . . . . . . . . . . . . . . . . 89

4.7.2. Tunability . . . . . . . . . . . . . . . . . . . . . . . . . . . . . . . . . . . . . . . . . .

4.7.3. Tunable Filter Parameters . . . . . . . . . . . . . . . . . . . . 90

4.7.4. Lumped Element Representations . . . . . . . . . . . . . . . . . . . 93

4.7.5. Piezoelectric LTCC Varactor Parameters . . . . . . . . . . . . . . . . . 95

4.8. Higher Order Filters . . . . . . . . . . . . . . . . . . . . . . . . . . . 98

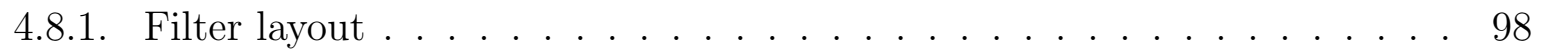

4.8.2. The Fabrication of the Filter . . . . . . . . . . . . . . . . . . . . . . 99

4.8.3. RF Measurements . . . . . . . . . . . . . . . . . . . . 101

4.8.4. Attenuation . . . . . . . . . . . . . . . . . . 104

4.8.5. Equivalent circuit . . . . . . . . . . . . . . . 105

$\begin{array}{lr}\text { 5. Interpretation } & 109\end{array}$

5.1. Performance Analysis . . . . . . . . . . . . . . . . . . . . . . . . 109

5.2. Piezoelectric Tuning Properties . . . . . . . . . . . . . . . . . . . 112

5.2.1. Tunable Coupling . . . . . . . . . . . . . . . . . . . . . . . . . . . . . . . . . . . . . . . .

5.2.2. Hysteresis Behaviour . . . . . . . . . . . . . . . . . 112

5.2.3. Tuning Sensitivity . . . . . . . . . . . . . . . . . 113

5.2.4. Power Consumptions . . . . . . . . . . . . . . . . . . 114

5.2.5. Tuning Speed . . . . . . . . . . . . . . . . 115

5.3. Competing Technologies . . . . . . . . . . . . . . . . 116

6. Outlook-Future work 117

6.1. Multiple tuned capacitances . . . . . . . . . . . . . . . . . . 117

6.2. Angular Misalignment . . . . . . . . . . . . . . . . . . . . . 120

6.3. Reproducibility, Reliability, and Fatigue . . . . . . . . . . . . . . . . 120 
$\begin{array}{lr}\text { 7. Conclusion } & 123\end{array}$

$\begin{array}{lr}\text { Appendices } & 126\end{array}$

$\begin{array}{lr}\text { A. Two-Port Parameters } & 127\end{array}$ 



\section{Introduction}

With growing number of supported frequency bands, the state of art of front ends not only leads to high cost and volume but also to poor performance due to the insertion loss of many cascaded stages and switches in the signal paths [1]. Thus frequency agility (tunability/reconfigurability) is the expected key feature of cost-efficient front end terminals.

Due to the urgent need for tunable filters, many design and material approaches have been described in literature. It is directly evident that a large gap exists between the tuning range needed from a systems point of view and those achieved by physical devices. A major research objective is the wide band RF front end covering the frequency range from $800 \mathrm{MHz}$ to 2500 $\mathrm{MHz}$ (tuning of $215 \%$ ) with properly scaled bandwidth and attenuation at higher frequency. The highest tuning ratios have been reported by Tombak et al. [2] (tuning of 57 \%), Yun et al. [3] (tuning of $24 \%$ ), and Sánchez-Renedo et al. [4] (tuning of $60 \%$ ).

Tunable devices should offer services which provide flexibility and scalability that match the system demands. For example, whatever the method of tuning may be, tunable filters must conserve as much as possible their transmission and reflection characteristics over a given tuning range. The tuning element plays an important role in determining the overall quality, sensitivity, size and power consumption of the tunable device and as a consequence the overall communication system performance.

Integrated voltage-controlled capacitors (varactors) are core components in tunable RF and microwave devices such as voltage controlled oscillators (VCO's), tunable filters, phase shifters, and tunable matching networks. A varactor with high quality factor and large tuning range is a mandatory prerequisite to meet the requirements of high tunable components specifications. For most of the tunable devices, it is not desired to make the device configuration complicated; other wise it will be hard to tune it from one frequency to another without perturbing the performance.

However, the design and manufacture of analogue filters with sufficient tuning range is not trivial and has not yet been satisfactoryly achieved to date due to the lack of a high quality tuning element. Thus, it calls for a joint effort in materials development, processing technologies and device concepts.

\subsection{State of The Art}

A variety of tuning-concepts have therefore been investigated over the years as described in [5]. Particularly noticeable are varactor diodes [6], paraelectric capacitors [7], and micro electromechanical system (MEMS) capacitors [8]. A specific characteristics of a varactor diode is its depletion region capacitance which can be varied by the applied bias voltage [9]. The capacitance is typically modeled as a parallel-plate capacitance with the depletion region serving as 
a dielectric. The depletion region varies with a corresponding change in voltage applied to the varactor diode, thereby changing the distance between the parallel plates and resulting in variable capacitance. Generally, the depletion region width is proportional to the square root of the applied voltage; and capacitance is inversely proportional to the depletion region width. Thus, the capacitance is inversely proportional to the square root of applied voltage. Semiconductors are limited in their applications by performance issues. Fundamentally, the quality factor $(Q)$ for varactor diodes varies inversely to the tunable capacitance range of the diode. The quality factor suffers from the high series resistance of the varactor diode junction, which results in lower quality when the diode capacitance is increased [10]. Therefore, circuits requiring high $Q$ values, such as wide tunable bandpass filter, do not rely on this sort of varactor. Kageyama et al. [11] have developed a tunable active filters with multilayer LTCC structure. The tunability was attained with the help of varactor diodes. A $13 \%$ tuning range of frequency at center frequency of $0.8 \mathrm{GHz}$ has been achieved. To avoid the decreases of the quality factor, the RF circuit has been adjusted to achieve low loss on the expense of the tuning range.

Paraelectric varactor [12], mainly Barium-Strontium-Titanate (BST) [13] rely on changing the dielectric constant of the BST material. BST is a nonlinear dielectric material, whose relative dielectric constant strongly depends on the electrical field strength in the material. The internal dielectric polarization can be changed, applying an external voltage on the material [14]. Due to the high electrical charge of the Ti-ion, a high dipole moment is induced, that results in both, a substantial absolute value and a relative change of the dielectric constant [15]. The dielectric loss tangent of BST films increases with frequency and depends on the film quality. Dielectric varactors based on tunable BST films are quite promising alternatives to semiconductor varactor diodes, in particular with increasing frequency [16]. Whereas the quality factor of semiconductor varactors decrease strongly with increasing frequency due to the dominating series resistance of the active semiconductor, the quality factor of a BST varactor is mainly determined by the film loss tangent [17]. Different microwave devices have been developed based on this valuable property. Examples of the applications of ferroelectric BST films include tunable resonators, filters (Tombak et al. [12]) and phase shifters (Dongsu et al. [18]), and variable frequency oscillator. There are also some products available by Paratek Microwave Inc. [19].

A MEMS varactor [20] closely resembles a traditional variable capacitor. In a MEMS varactor, the distance between capacitor plates is varied with a control voltage, thus changing the capacitance [21]. The voltage across the electrodes is varied to pull down and up membrane, which varies the distance [22]. The capacitance is tuned by varying the air gap, or the overlap area, or both simultaneously by actuation. Compared with solid-state varactors, micromachined tunable capacitors have lower loss and potentially lower tuning range due to the pull-in effect and parasitic capacitance [23]. A problem in MEMS devices is stiction [24]. However, a significant drawback is the highly nonlinear tuning response as a function of the actuation of the device [25]. Several driving principles which are suitable in the micro domain are used including electrostatic [26], piezoelectric [27] and thermal [28] actuation. Each mechanism has specific advantages with respect to deflection range, required force, power requirements, and repones time. A wide variety of MEMS tunable capacitors have been reported in literature: An electro-thermal actuator has been used for driving the top plate of the parallel plate capacitor 
in the work of Feng et al., [29]. The measured $Q$ is 256 at $1 \mathrm{GHz}$ and a tuning ratio of 2 to 1 has been reported. A high $Q$ tunable micromechanical capacitor has been developed by Yoon et al., [30], the key feature in this design based on moving the dielectric between the capacitor plates, rather than moving the plates themselves. A measured $Q$ of 291 at $1 \mathrm{GHz}$ with a tuning range $7.7 \%$ over $10 \mathrm{~V}$ has been reported. Wang et al., [31], used a suspended plate array and bottom array to tune an interdigitated comb capacitance. A micromachined parallel plate tunable capacitor consists of one suspended top plate and two fixed bottom plates has been presented by Zou et al., [32], for the fabricated prototype, a maximum tuning range of $69 \%$ has been achieved. A MEMS capacitor has been fabricated in a thin film technology using a dual gap relay type design by Rijks et al., [33], the capacitor shows a continuous and reversible capacitance tuning with a tuning ratio up to 17 ( the capacitance tuned roughly from $0.9 \mathrm{pF}$ to $0.05 \mathrm{pF}$ ), while requiring an actuation voltage of only $20 \mathrm{~V}$. A quality factor of 150 to 500 has been measured in the frequency range of 1 to $6 \mathrm{GHz}$. Park et al., [34], fabricated a micromachined RF MEMS tunable capacitor using piezoelectric actuator. The fabricated device has a tuning ratio of 3.1 to 1 at bias voltage of $6 \mathrm{~V}$ and a quality factor of 210 at $1 \mathrm{GHz}$.

All of these approaches come with their individual benefits and draw-backs in terms of power consumption, speed, reliability, microwave losses or drive voltage level. These approaches are not feasible for a broadband tuning ranges. Therefore it is fundamentally impossible to realize a large tuning ratio combined with an appreciable quality factor.

\subsection{Objective}

The next generation of cellular phone terminals will require coverage of a significantly larger number of bands. The total spectrum allocated to these services amounts to more than 2 $\mathrm{GHz}$ (including occasional overlap of the bands of different bandwidths). Furthermore new approaches to spectrum disposition are prepared at regulatory agencies including dynamic spectrum allocation and opportunistic use of spectrum which will disrupt today's fixed oneto-one mapping between communication standards and frequency bands [35]. Thus, frequency agility is one of the expected key features of future terminals. Furthermore, adaptable or tunable analogue RF front end sections within these devices could lead to fully software controlled operation, modification and updates. The notion "software-defined radio" (SDR) has been coined for this idea some time ago.

If a software defined radio (SDR) could be implemented in hardware, this would allow the wireless transmission and reception of signals of any frequency, power level, bandwidth, and modulation technique. A multi-100Gb/s ADC/DAC connected to a suitable antenna would be the ideal realization performing all of up/down conversion, filtering and baseband processing in the digital regime. However, such ADC performance will not become available in the foreseeable future. The SDR frontend (FE) therefore has to be split into a digital section DFE extending from the baseband processor as far as technically feasible towards the antenna, including occasionally the intermediate frequency (IF) functions (see Fig. 1.1), and an analog RF section AFE bridging the gap between the highest frequency digital functions and the antenna [36]. Current agile frontend concepts achieve tunability by a multitude of tunable frequency selective elements, e.g. matching networks, switches, power amplifier. Antenna switch modules 
(ASM) [37] and switchable filter banks [38] are more easier to realize. They are not exhibit stepwise tuning, but also poor performance due to the insertion loss of many cascaded stages and switches in the signal paths and they are usually not small. They are limited to a small number of fixed transmission, $T x$, and reception, $R x$, bands.

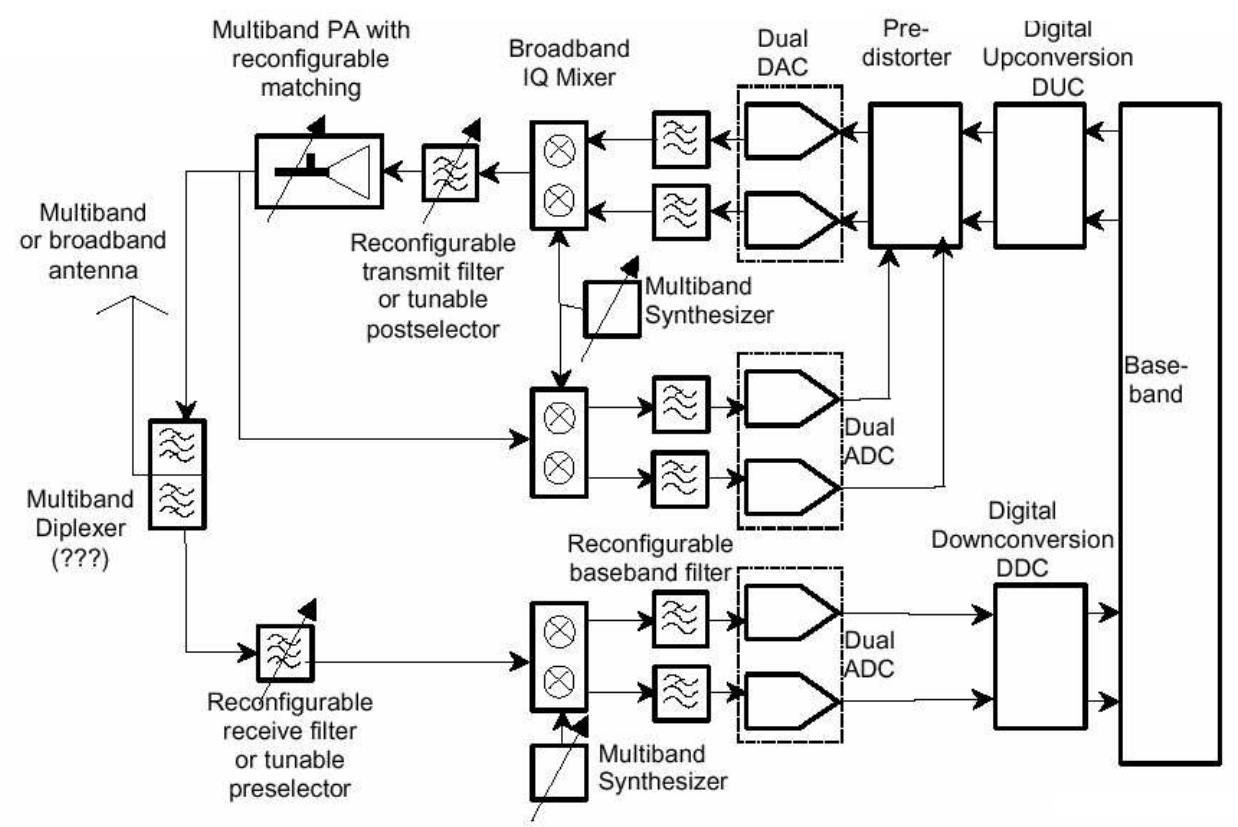

Figure 1.1.: SDR system architecture (After Lucent Technologies).

The lack of frequency agility in the RF front-end is in fact the major technological blocking point on the evolution towards multi-standard enabled terminals and cost effective platform designs. For the foreseeable future, there will always be some analogue components on the high-frequency side of the frontend. Making these adaptable to various modes and tunable to various frequencies will pave the way for a fully software controlled or software defined radio [39]. A single filter with wide tuning range (or frequency shift of its pass band which can be controlled by an external voltage) would enable radio manufacturers to replace several fixed filters covering adjacent frequencies [40]. This versatility provides RF front-end tunability in real time applications and decreases deployment and maintenance costs through software controls and reduced component count. However, the design and manufacture of analogue filters with sufficient tuning range is not trivial and has not yet been satisfactorily achieved to date. A number of approaches has been tested in the community, largely based on semiconductor varactor diodes, tunable ferroelectric materials, and micro-electromechanical systems (MEMS), but to our knowledge severe penalties always come along with moderately promising performance. The present approach offers innovative tunability performance that combines favorable properties with respect to tuning range, quality factor and power consumption. 


\subsection{Outline}

The task of the present work is to study the performance potential of piezoelectrically driven micromechanically tuned band pass filters in the frequency range of wireless AFEs and to assess this device concept with respect to its design reliability, technical feasibility and mass producibility.

After the short review of the state-of-the-art in tuning in chapter one, several potentially attractive concepts are studied with attention to a maximal tuning range by using commercial software for circuit simulation as well as 2.5D and 3D electromagnetic device simulation.

Chapter two contains an overview of the published network synthesis techniques for filter design theory. The design is simplified by beginning with low pass filter prototypes that are normalized in terms of impedance and frequency. Frequency transformations are then applied to convert the prototype designs to the desired frequency range and impedance level. Also this chapter summarizes the design procedures for tunable combline filters as they are described in traditional literature in order to make them applicable to the present design goal. The design procedure starts with the specification of the desired filter characteristics over the tuning range. The proposed method gives straightforward criteria to choose the filter design parameters that lead to the determination of the self- and mutual capacitances per unit length of the coupled microstrip lines. These circuits elements are used to compute the normalized coupling between the coupled lines. Next, the filter dimension are constructed.

The technology for the fabrication and assembly issues arising from the device structure which combines the two ceramics components, LTCC and piezoelectric actuator technologies have been covered in chapter three. In order to guarantee optimum performance of both, the LTCC integrated coupled line filter elements and the piezoelectric element, at hybrid assembly process of two separately fabricated and optimized components is adopted here. The characterization of the deflection of the cantilever glued to LTCC substrate is also presented. It has been measured using an optical measurements setup as a function of tuning. It is based on triangulation principle.

The study in chapter one has lead to a novel piezoelectrically driven variable capacitor with wide tuning range. The potential of this approach is presented in chapter four. The proposed capacitor is tuned by varying the gap width between the electrodes. In contrast to the conventional two parallel plate capacitors, the present approach employs a piezoceramic cantilever to move the top electrode. This varactor can be further integrated with passive components to yield specific frequency-tunable characteristics. After the setup for the RF measurements, the fabricated device performance is studied. The center frequency of the filter is tuned from $1.1 \mathrm{GHz}$ to $2.6 \mathrm{GHz}$ with $200 \mathrm{~V}$ control voltage and low insertion loss value of $4 \mathrm{~dB}$ (at zero-bias) to $2 \mathrm{~dB}$ (at the maximum-bias. The present filter design focuses on the demonstration of feasibility, tuning range, and device compactness. Although no optimization was done for power consumption, tuning speed, drive voltage, filter attenuation, and insertion loss. This chapter also shows the effects of tuning mechanism on the overall quality factor, return loss, insertion loss, and the relative bandwidth at the mid of the band as a function of frequency across the entire tuning range. As with any other filter technology, quality factor and selectivity can be improved at the expense of insertion loss, e.g. by adding further circuit 
elements or resonators. A second order combline filter has been designed and fabricated in this chapter. This chapter treats also the measurements and characterizations of this filter. The filter has shown a better selectivity of 10 to $15 \mathrm{~dB}$ more than the first order. The quality factor of the capacitor has been improved by a factor of 2 for a cantilever metallization of $150 \mathrm{~nm}$ gold.

The measurement results have been analyzed and interpreted in chapter five. The device shows a word record tuning range and a relative low insertion loss with respect to the tunable filters were published. The analysis of the device by full-wave simulation reveals a potential tuning range from $0.8 \mathrm{GHz}$ to $2.8 \mathrm{GHz}$ when the thin-film processability of the LTCC surface is properly controlled. Switching speed, dynamic behaviors as well as power consumption are being addressed in this chapter. The device outlook is also presented in this chapter.

The idea of the present approach that has been explained in the fourth chapter could be extended to tune multiple capacitors with a single cantilever in chapter six. The filter topology plays the most important role in doing that. The major challenge would be where to place the capacitors with in the filter structure for optimum tunability. In higher-order version of the present structure (combline filter) a set of cantilevers will be driven simultaneously and individually to tune the filter. Throughout this chapter a proposed solution which looks feasible will be studied. Also, the angular misalignment, reproducibility, reliability, and fatigue are shortly addressed.

Chapter seven concludes this thesis by summarizing and discussing its main results. 


\section{Fundamentals of Tunable Filter Theory}

A microwave filter is a two-port network used to control the frequency response at a certain point in a microwave system by providing transmission at frequencies within the passband and attenuation in the stopband [41]. The main types of microwave filters are: waveguide filters [42] (characterized by high power, low loss and large size), dielectric resonator filters [43] with a quality factor $Q \sim 10.000$ (low loss and small size), and filters based on planar structures, such as microstrip lines [44]. Most of the typical microwave filters used today are summarized in [45]. A filter design needs to take into accounts physical concerns such as size, weight, and cost, as well as performance considerations, including isolations, loss minimization, and group delay. Fig. 2.1 shows the amplitude response of a theoretical bandpass filter. This figure serves

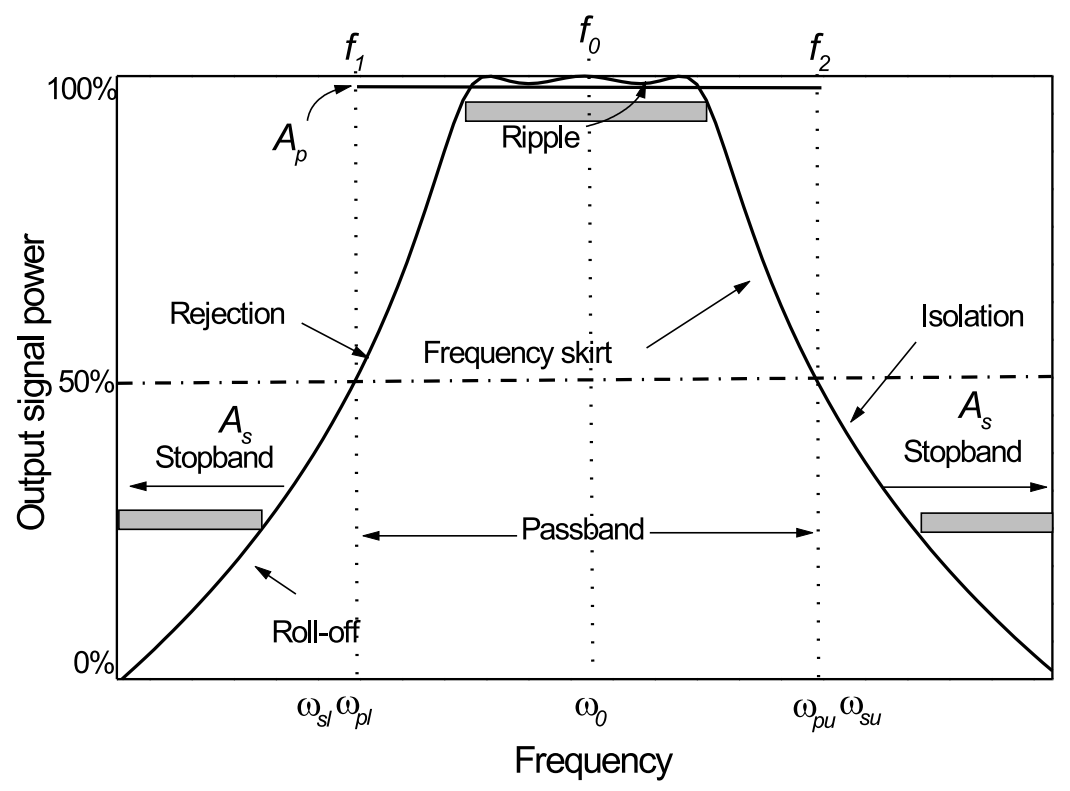

Figure 2.1.: Frequency response, theoretical bandpass filter.

to define the following parameters: the minimum passband gain $A_{p}$, the maximum stopband gain $A_{s}$, the center frequency $\omega_{0}$, upper passband and stopband frequencies $\omega_{p u}$ and $\omega_{s u}$ and lower passband and stopband cutoff frequencies $\omega_{p l}$ and $\omega_{s l}$. Frequency ranges where the gain is relatively large are called passbands and those where the gain is relatively small, are stopbands. Those in between where the gain increasing or decreasing are termed transition bands. Almost always one parameter has to be scarified a little bit in order to improve another, such the tradeoff of insertion loss and selectivity. 
Filters can be designed using the image parameter [46] or the insertion loss methods [47]. The image impedance and attenuation function of a filter section are defined in terms of an infinite chain of identical filter sections connected together [48]. The image parameter method may yield a usable filter response, but if not there is no clear cut-way to improve the design. Derivations for the design equations and more complete discussions can be found in [49].

\subsection{Network Synthesis Method}

Network synthesis methods [50] start out with a completely specified frequency response. The design is simplified by beginning with low pass filter prototypes that are normalized in terms of impedance and frequency. Transformations are then applied to convert the prototype designs to the desired frequency range and impedance level. The insertion loss allows filter performance to improve in a straightforward manner, at the expense of a higher order filter.

\subsubsection{Synthesis Techniques}

A two-port network can be synthesized from its impedance function $Z(p)$, admittance $Y(p)$ or its scattering parameters $S(p)$ [51], where $p$ is a normalized complex frequency variable given by

$$
p=\sigma+\mathrm{j} \omega
$$

In passive network synthesis techniques it is often desirable to work with the network reflection coefficients rather than the input impedance. Since synthesis procedure requires the availability of rational functions, the generating of these procedure for generating these functions will be briefly discussed. Suppose that $S_{11}(p)$ could be written as fractional polynomial:

$$
S_{11}(p)=\frac{N(p)}{D(p)}
$$

The objective now is to determine $S_{11}(\mathrm{j} \omega)$ by its values along the $\mathrm{j} \omega$ axis. This done by the determination of the numerator and denominator coefficients to meet the stated specifications. Once this accomplished, the computation of the transfer function is processed in order to synthesized the filter. The function in (2.2) is synthesizable into network only if it is a positive real function [52] this means that its input impedance $Z_{\text {in }}(p)$ (or the input admittance $Y_{\text {in }}(p)$ ) is real, thus require $p$ to be real, mathematically

$$
\Re\left\{Z_{\text {in }}(p)\right\}>0 \text { for } \Re\{p\}>0
$$

or

$$
\Re\left\{Y_{\text {in }}(p)\right\}>0 \text { for } \Re\{p\}>0
$$


Since $Z_{\text {in }}(p)$ (or $Y_{i n}(p)$ ) is a positive real function, thus $S_{11}(p)$ is a bounded real function, mathematically

$$
\left|S_{11}(p)\right|<1 \text { for } \Re\{p\}>0
$$

i.e. $S_{11}(p)$ is analytic function and contains no poles or zeros in the right half-plane of $p$. The poles and zeros in (2.2) are either real or complex conjugate pairs. Thus, all the coefficients of $N(p), D(p)$ are real and positive with zeros and poles on the $\mathrm{j}$-axis in the $\sigma-\omega$ plane. From ( 2.3) ( or $(2.4)$ ), $Z_{i n}(p)$ (or $Y_{i n}(p)$ ) has no poles or zeros in the right half-plane. Since $S_{11}(p)$ is analytic function [53] then the complex conjugate satisfies $S_{11}^{*}(p)=S_{11}\left(p^{*}\right)$, therefore

$$
\left[S_{11}(p) S_{11}(-p)\right]_{p=\mathrm{j} \omega}=S_{11}(\mathrm{j} \omega) S^{*}{ }_{11}(\mathrm{j} \omega)=\left|S_{11}(\mathrm{j} \omega)\right|^{2}
$$

This yields $\left|S_{11}(\mathrm{j} \omega)\right|^{2}$ to be function in terms of $\omega^{2}$. Therefore

$$
\left|S_{11}(\mathrm{j} \omega)\right|^{2}=A^{2}\left(\omega^{2}\right)
$$

where $A^{2}\left(\omega^{2}\right)$ is a real polynomial in terms of $\omega^{2}$ and could be characterized by the ratio of two polynomials in $\omega^{2}$ such as $B\left(\omega^{2}\right)$ and $C\left(\omega^{2}\right)$ where $A^{2}\left(\omega^{2}\right)$ is given by

$$
A^{2}\left(\omega^{2}\right)=\frac{B\left(\omega^{2}\right)}{C\left(\omega^{2}\right)}
$$

Since

$$
S_{11}(p) S_{11}(-p)=\frac{N(p) N(-p)}{D(p) D(-p)}
$$

Therefore

$$
[N(p) N(-p)]_{p=\mathrm{j} \omega}=N(\mathrm{j} \omega) N^{*}(\mathrm{j} \omega)=|N(\mathrm{j} \omega)|^{2}=B\left(\omega^{2}\right)
$$

and the same for $D(p)$

$$
[D(p) D(-p)]_{p=\mathrm{j} \omega}=D(\mathrm{j} \omega) D^{*}(\mathrm{j} \omega)=|D(\mathrm{j} \omega)|^{2}=C\left(\omega^{2}\right)
$$

Since (2.7) requires the substitutions $p \rightarrow \mathrm{j} \omega$, one also has $p^{2} \rightarrow-\omega^{2}$, and conversely. Thus

$$
S_{11}(p) S_{11}(-p)=A^{2}\left(-p^{2}\right)
$$


The problem now is to compute $N(p), D(p)$ from ( 2.10) and (2.11) respectively. So the problem is turn to find $N(p)$ from $N(p) N(-p)$, and finding $D(p)$ from $D(p) D(-p)$. To illustrate this procedure, assume that $\left|S_{11}(\mathrm{j} \omega)\right|^{2}$ is given by

$$
\left|S_{11}(\mathrm{j} \omega)\right|^{2}=\frac{\omega^{6}}{1+\omega^{6}}
$$

From (2.7)

$$
A^{2}\left(\omega^{2}\right)=\frac{\omega^{6}}{1+\omega^{6}}
$$

Therefore from $(2.8)$

$$
\begin{aligned}
& B\left(\omega^{2}\right)=\omega^{6} \\
& C\left(\omega^{2}\right)=1+\omega^{6}
\end{aligned}
$$

Applying (2.12)

$$
A^{2}\left(-p^{2}\right)=\frac{\left(-p^{2}\right)^{3}}{1+\left(-p^{2}\right)^{3}}
$$

Therefore from (2.8)

$$
\begin{aligned}
& B\left(-p^{2}\right)=\left(-p^{2}\right)^{3} \\
& C\left(-p^{2}\right)=1+\left(-p^{2}\right)^{3}
\end{aligned}
$$

Now, both functions $N(p)$ and $D(p)$ will be find by solving the next two equations simultaneously:

$$
\begin{aligned}
& {[N(p) N(-p)]_{p=\mathrm{j} \omega}=\omega^{6}=\left(-p^{2}\right)^{3}} \\
& {[D(p) D(-p)]_{p=\mathrm{j} \omega}=1+\omega^{6}=1+\left(-p^{2}\right)^{3}}
\end{aligned}
$$

more simplifications

$$
\begin{aligned}
& {[N(p) N(-p)]_{p=\mathrm{j} \omega}=-p^{6}} \\
& {[D(p) D(-p)]_{p=\mathrm{j} \omega}=1-p^{6}}
\end{aligned}
$$

the solutions are 


$$
N(p)= \pm p^{3}
$$

and

$$
[D(p) D(-p)]_{p=\mathrm{j} \omega}=1-p^{6}
$$

A process known as the factorization problem which yields a non-unique solution is used to write $D(p) D(-p)$ as a product of two functions. One of these functions $D(p)$ is assigned all left-hand of plane (LHS) poles while the other $D(-p)$ is assigned all right-hand of plane (RHS) poles.

$$
\left(1-p^{6}\right)=\left(1+2 p+2 p^{2}+p^{3}\right)\left(1-2 p+2 p^{2}-p^{3}\right)
$$

$\left(1-p^{6}\right)$ is expanded to two polynomials multiplied with each other. The stability of the network requires that $D(p)$ has a real coefficients which is bigger than zero, to ensure that

$$
D(p)=\left(1+2 p+2 p^{2}+p^{3}\right)
$$

Therefore

$$
S_{11}(p)=\frac{ \pm p^{3}}{1+2 p+2 p^{2}+p^{3}}
$$

Since

$$
Z(p)=\frac{1+S_{11}(p)}{1-S_{11}(p)}
$$

Therefore

$$
Z(p)=\frac{D(p)+N(p)}{D(p)-N(p)}
$$

Finally, two possible solutions are available for $Z(p)$, for $N(p)=-p^{3}$, which is

$$
Z(p)=\frac{1+2 p+2 p^{2}}{1+2 p+2 p^{2}+2 p^{3}}
$$

and the other for $N(p)=+p^{3}$, which is

$$
Z(p)=\frac{1+2 p+2 p^{2}+2 p^{3}}{1+2 p+2 p^{2}}
$$

Hence, there are two possible realization for this network. 


\subsubsection{Ladder Network}

A common realization of the previous impedance functions (2.32)and (2.33) used in filter design is the ladder network shown in Fig. 2.2. The ladder network can has four forms, beginning with a series elements and ending with a shunt element, or beginning with a series element and ending with a series element like in Fig. 2.2. The other two possibilities is to begins with a shunt element and ends with series or shunt element, respectively. The input impedance of Fig. 2.2 is given by the continued fraction expansion

$$
\begin{aligned}
& Z_{\text {in }}(p)=p Z_{1}+\frac{1}{p Z_{2}+\frac{1}{p Z_{3}+}} \\
& +\frac{1}{p Z_{N-1}+\frac{1}{p Z_{N}}}
\end{aligned}
$$

where $Z_{i}$ represent an inductor or capacitor. Since the impedance numerator degree of (2.32) is less than the denominator degree, the corresponding ladder network will start with a shunt capacitor element. The value of this capacitor is computed by evaluate the residue of $Y(p)$ at $p=\infty$. Thus

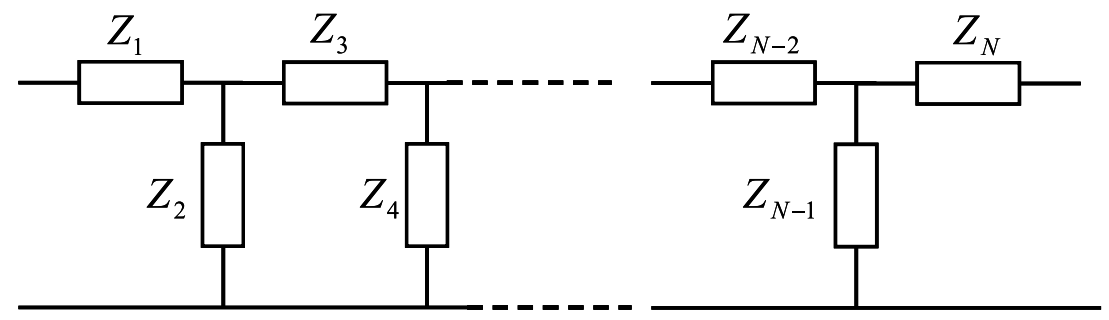

Figure 2.2.: The ladder network.

$$
Y(p)=\frac{1+2 p+2 p^{2}+2 p^{3}}{1+2 p+2 p^{2}}
$$

the residue evaluate by dividing the admittance over $p$ and taking the limit as $p$ tends to $\infty$, i.e.

$$
\left[\frac{Y(p)}{p}\right]_{p=\infty}=\lim _{p \rightarrow \infty} \frac{1+2 p+2 p^{2}+2 p^{3}}{p+2 p^{2}+2 p^{3}}=\lim _{p \rightarrow \infty} \frac{\frac{1}{p^{3}}+\frac{2}{p^{2}}+\frac{2}{p}+2}{\frac{1}{p^{2}}+\frac{2}{p}+2}=1
$$

Now the shunt capacitor of value $1 \mathrm{~F}$ is removed from admittance function, leaving the remaining admittance $Y_{1}(p)$

$$
Y_{1}(p)=Y(p)-1 p=\frac{1+2 p+2 p^{2}+2 p^{3}}{1+2 p+2 p^{2}}-p=\frac{1+p}{1+2 p+2 p^{2}}
$$


Since the admittance numerator degree is bigger than the denominator degree in the impedance function $Y_{1}(p)$ the next element will be a series inductor. Again, the residue of $Y_{1}(p)$ is evaluated at $p=\infty$

$$
\begin{gathered}
Z_{1}(p)=\frac{1}{Y_{1}(p)}=\frac{1+2 p+2 p^{2}}{1+p} \\
{\left[\frac{Z_{1}(p)}{p}\right]_{p=\infty}=\lim _{p \rightarrow \infty} \frac{1+2 p+2 p^{2}}{p+p^{2}}=2}
\end{gathered}
$$

so the value of the series inductor of value $2 \mathrm{H}$ will be extracted from $Z_{1}(p)$, leaving a remanning impedance $Z_{2}(p)$

$$
Z_{2}(p)=Z_{1}(p)-2 p=\frac{1}{1+p}
$$

now

$$
Y_{2}(p)=\frac{1}{Z_{2}(p)}=1+p
$$

Again, the residue of $Y_{1}(p)$ is evaluated at $p=\infty$

$$
\left[\frac{Y_{2}(p)}{p}\right]_{p=\infty}=\lim _{p \rightarrow \infty} \frac{1}{p}+1=1
$$

Again, the shunt capacitor of value $1 \mathrm{~F}$ is removed from admittance function, leaving the remaining admittance $Y_{3}(p)$

$$
\begin{aligned}
Y_{3}(p) & =Y_{2}(p)-1 p \\
& =1
\end{aligned}
$$

$Y_{3}(p)$ represent the load resistor of value $1 \Omega$. The complete synthesis process is shown in Fig. 2.3(a). Also, Fig. 2.3(b) shows that one for (2.33). 


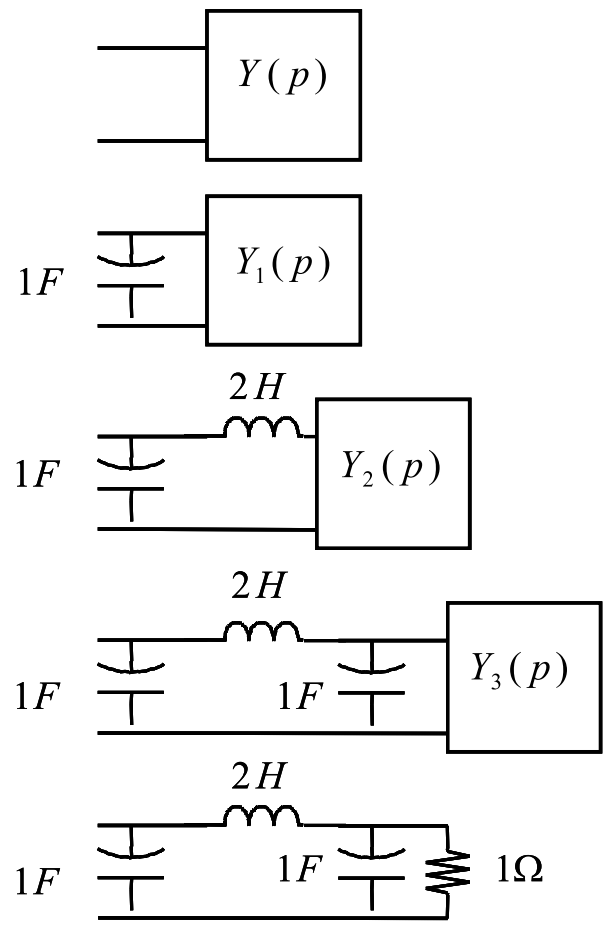

(a) The synthesis procedure of the impedance function (2.32).
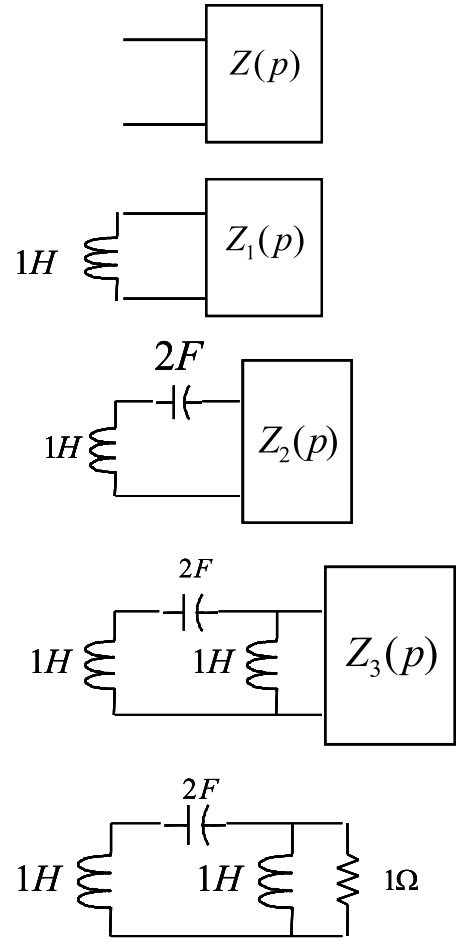

(b) The synthesis procedure of the impedance function (2.33).

Figure 2.3.: The complete synthesis process 


\subsubsection{Losses of Two-Port Network}

In this subsection the definitions of insertion and return losses within a general two-port network is introduced. Consider Fig. 2.4, where is the $\left(P_{I n}\right)$ is the incident power, $\left(P_{R}\right)$ the power reflected back to the generator, $\left(P_{L}\right)$ the power absorbed by the filter, $\left(P_{A}\right)$ the power transmitted to the load. The insertion loss (in decibels) at a particular frequency can be defined as:

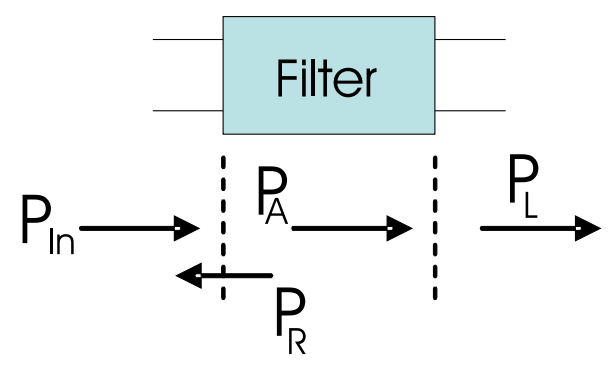

Figure 2.4.: General filter network configuration.

$$
P_{I n}=P_{R}+P_{L}
$$

The insertion loss $L_{A}$ of the filter is defined by the ratio of the power available from the source to the power delivered to the load, mathematically:

$$
L_{A}=\frac{P_{I n}}{P_{L}}=\frac{1}{1-\left|S_{11}(\mathrm{j} \omega)\right|^{2}}=\frac{1}{\left|S_{12}(\mathrm{j} \omega)\right|^{2}}
$$

In $d B$ the insertion loss is expressed by

$$
\left[L_{A}\right]_{d B}=-20 \log _{10}\left|S_{12}(\mathrm{j} \omega)\right| \quad d B
$$

This value represents the transfer function $S_{12}(p)$ expressed in decibels. The reflection coefficient $S_{11}(p)$ expressed in decibels and is known as the return loss $L_{R}$. The return loss $L_{R}$ of the filter is defined by the ratio of the power available from the source to the power reflected from the load, mathematically:

$$
L_{R}=\frac{P_{I n}}{P_{R}}=\frac{1}{1-\left|S_{12}(\mathrm{j} \omega)\right|^{2}}=\frac{1}{\left|S_{11}(\mathrm{j} \omega)\right|^{2}}
$$

In $d B$ the return loss is expressed by 


$$
\left[L_{R}\right]_{d B}=-20 \log _{10}\left|S_{11}(\mathrm{j} \omega)\right| \quad d B
$$

The insertion loss is a measure of the attenuation through the network. The return loss is a measure of how well matched the network is. A perfectly matched lossless network would have zero insertion loss and infinite return loss. In practice, a polynomial transfer function as Butterworth [54], Chebyshev [55], and Bessel [56] is used to model the filter response.

\subsection{Lowpass Prototype Filter}

The lowpass prototype which may be of lumped or distributed realization from which real filters may be constructed. Frequency transformation allows to derive highpass, bandpass and bandstop from the lowpass prototype. As a result the following transformations are used [57]

$$
\begin{array}{ll}
(l p p \leftrightarrow l p) & : \quad s \rightarrow k p \\
(l p p \leftrightarrow h p) \quad: \quad s \rightarrow \frac{k}{p} \\
(l p p \leftrightarrow b p) \quad: \quad s \rightarrow \frac{p^{2}+\omega_{0}}{\Delta \omega p} \\
(l p p \leftrightarrow b s) \quad: \quad s \rightarrow \frac{\Delta \omega p}{p^{2}+\omega_{0}}
\end{array}
$$

where $k, \Delta \omega$ and $\omega_{0}$ are the scaling factor, the passband bandwidth and the center frequency, respectively. Fig.2.5 shows how the lowpass prototype convert to other types.

\subsubsection{Butterworth Filters}

The butterworth filter is also known as the maximally flat due to the fact it has the most flat passband response. This kind is useful if the signal distortion in the passband must be kept at the minimum. The filter response is less steep compared with other filters type. Therefore, this kind of filter is less useful for filtering signals which are located closely to each other in the spectrum. The magnitude response of $N$ order butterworth filter is given by

$$
|H(\mathrm{j} \omega)|=\frac{1}{\sqrt{1+\omega^{2 N}}}
$$

Thus

$$
\left|S_{12}(\mathrm{j} \omega)\right|^{2}=\frac{1}{1+\omega^{2 N}}
$$

The order $N$ is determine from the equation 


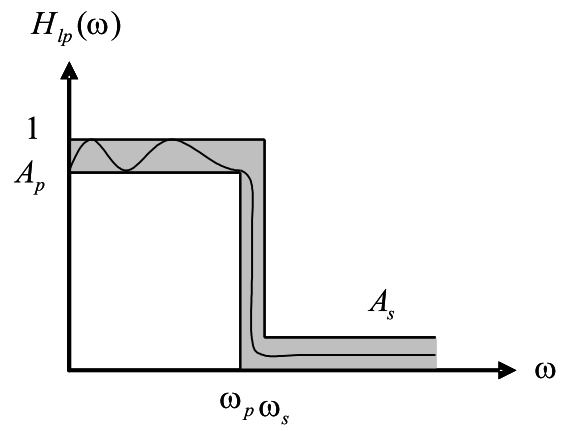

(a) Lowpass filter.

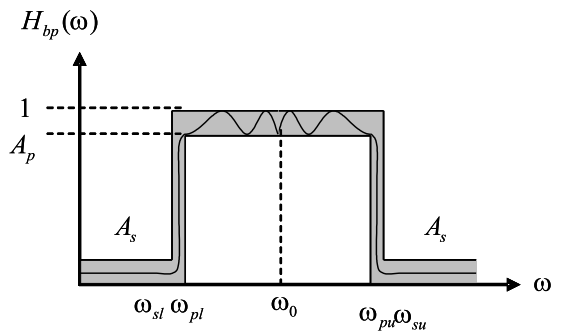

(c) Bandpass filter.

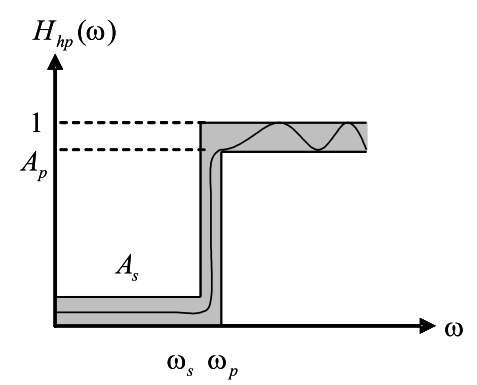

(b) Highpass filter.

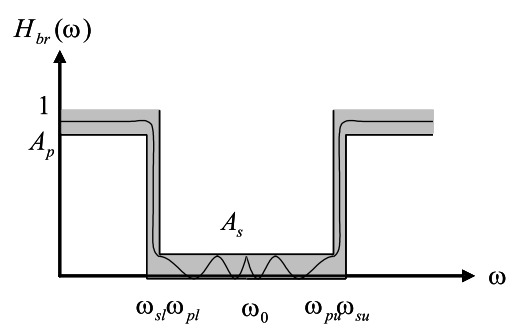

(d) Bandstop filter.

Figure 2.5.: Catalog of basic filter types

$$
\frac{1}{\sqrt{1+\omega^{2 N}}} \leq A_{s}
$$

or, rearrange;

$$
N \geq \frac{\log \left[\frac{1}{A_{s}^{2}}-1\right]}{2 \log \left(\omega_{s}\right)}
$$

The value of $N$ is chosen to be the smallest integer greater than the expression on the right-hand side of (2.58). The synthesis of the maximally flat filter proceeds as follows:

Since

$$
\left|S_{11}(\mathrm{j} \omega)\right|^{2}=\frac{\omega^{2 N}}{1+\omega^{2 N}}
$$

Therefore

$$
S_{11}(\mathrm{j} \omega) S_{11}(-\mathrm{j} \omega)=\frac{\omega^{2 N}}{1+\omega^{2 N}}
$$

To construct the $S_{11}(p) S_{11}(-p)$, the $\omega^{2}$ is replaced by $-p^{2}$ 


$$
S_{11}(p) S_{11}(-p)=\frac{\left(-p^{2}\right)^{N}}{1+\left(-p^{2}\right)^{N}}
$$

Therefore from (2.8) we obtain

$$
\begin{aligned}
& B\left(-p^{2}\right)=\left(-p^{2}\right)^{N} \\
& C\left(-p^{2}\right)=1+\left(-p^{2}\right)^{N}
\end{aligned}
$$

Now, both functions $N(p)$ and $D(p)$ will be find by solving the next two equations simultaneously:

$$
\begin{aligned}
& {[N(p) N(-p)]_{p=\mathrm{j} \omega}=\left(-p^{2}\right)^{N}} \\
& {[D(p) D(-p)]_{p=\mathrm{j} \omega}=1+\left(-p^{2}\right)^{N}}
\end{aligned}
$$

The resulting filter should be stable. This means that the poles of $S_{11}(p) S_{11}(-p)$ that lie in the right-half plane must be discarded and the remaining ones assigned to $S_{11}(p)$. Solving (2.64) yields

$$
N(p)= \pm p^{N}
$$

Now, $D(p)$ should be formed from the left half-plane poles, i.e. the zeros of the left half-plane could be found as follows: since

$$
[D(p) D(-p)]_{p=\mathrm{j} \omega}=1+(-1)^{N}(p)^{2 N}
$$

The problem now is merely to find all the poles of $S_{11}(p) S_{11}(-p)$, then to sort them. These poles are located at the zeros of the denominator in (2.9). Thus, one must solve

$$
1+(-1)^{N}(p)^{2 N}=0
$$

this implies that

$$
p^{2 N}=(-1)^{N-1}=\mathrm{e}^{\mathrm{j} \pi(N-1)}
$$

Representing $p$ in polar coordinates by

$$
p=\rho \mathrm{e}^{\mathrm{j} \phi}
$$


Therefore (2.69)becomes

$$
\rho^{2 N} \mathrm{e}^{\mathrm{j} 2 N \phi}=\mathrm{e}^{\mathrm{j} \pi(N-1)}
$$

This has the solution

$$
\rho=1
$$

and

$$
\phi=\frac{\pi(N-1)}{2 N}+\frac{2 r \pi}{2 N}=\frac{\pi}{2}+\frac{\pi(2 r-1)}{2 N}
$$

where $r$ is any integer. Now, since $\mathrm{e}^{\mathrm{j} \frac{\pi}{2}}=\mathrm{j}$ and assuming that

$$
\theta_{r}=\frac{(2 r-1) \pi}{2 N}
$$

Therefore

$$
p_{r}=\mathrm{je}^{\mathrm{j} \theta_{r}}=-\sin \left(\theta_{r}\right)+\mathrm{j} \cos \left(\theta_{r}\right) \quad r=1, \ldots, 2 n
$$

Since

$$
p_{r}=\sigma_{r}+\mathrm{j} \omega_{r}
$$

Therefore

$$
\begin{aligned}
& \sigma_{r}=-\sin \left(\theta_{r}\right) \\
& \omega_{r}=\cos \left(\theta_{r}\right)
\end{aligned}
$$

Now since

$$
\cos ^{2}\left(\theta_{r}\right)+\sin ^{2}\left(\theta_{r}\right)=1
$$

That is;

$$
\sigma_{r}^{2}+\omega_{r}^{2}=1
$$




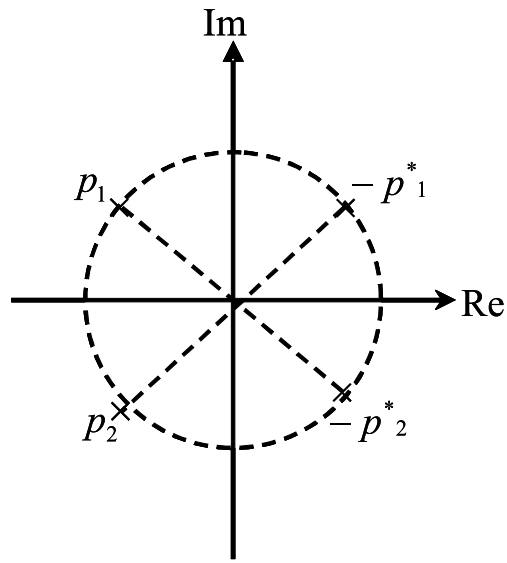

(a) Projection on the $\sigma-\omega$ plane.

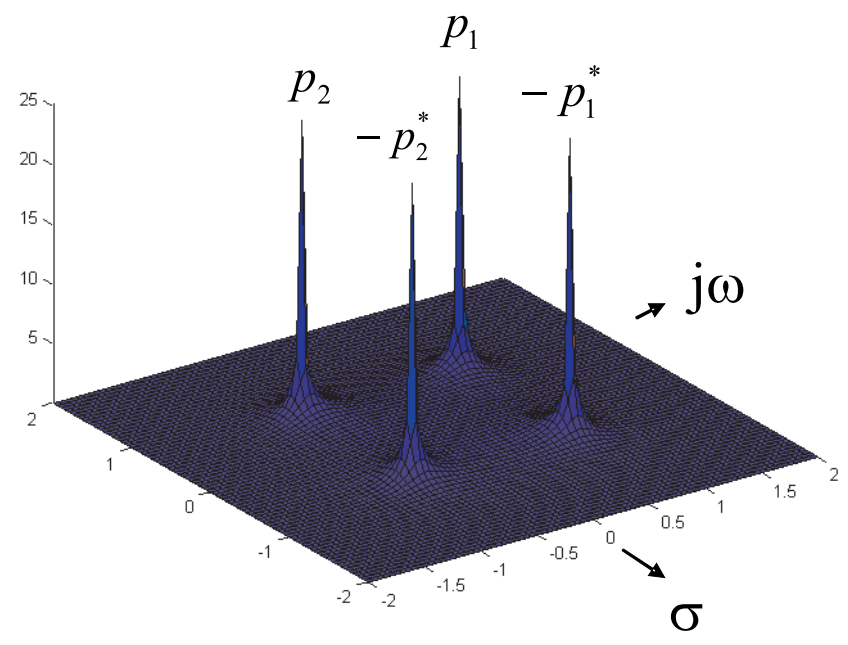

(b) $3 \mathrm{D}$ view of the poles.

Figure 2.6.: Poles of the second order Butterworth transfer function.

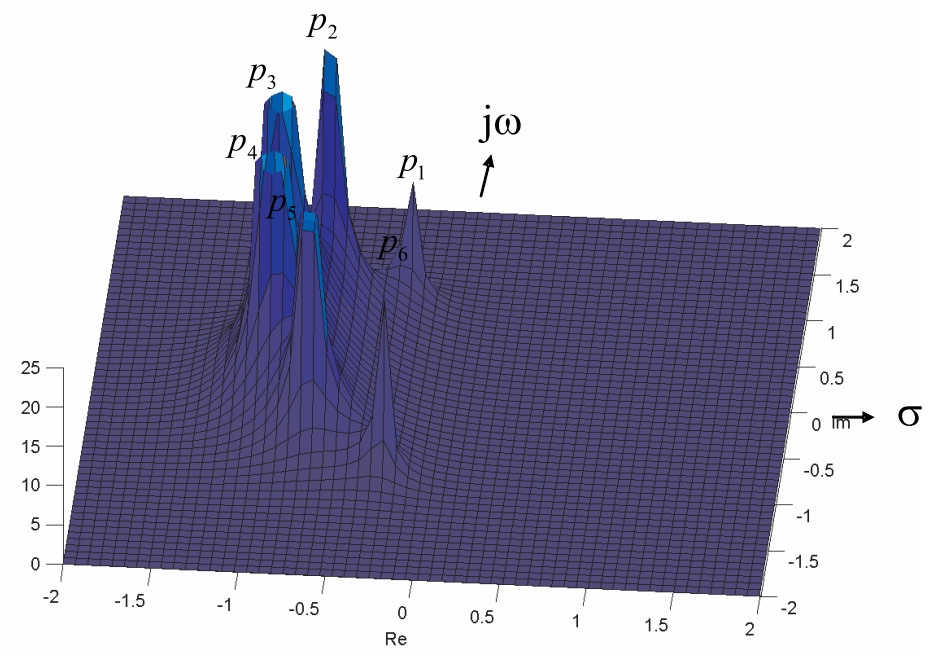

Figure 2.7.: Poles of the second order Butterworth. 
Thus, the poles of the lowpass prototype utilizes a Butterworth functions lie on a unit circle in the complex plane and the first $n$ roots lie in the half-plane. For second order butterworth the poles are shown in Fig. 2.6(b), where $p_{1}=-0.7071+\mathrm{j} 0.7071, p_{2}=-0.7071-\mathrm{j} 0.7071$, $-p_{1}^{*}=+0.7071+\mathrm{j} 0.7071,-p_{2}^{*}=+0.7071+\mathrm{j} 0.7071$. For the $6^{\text {th }}$ order butterworth the poles are shown in Fig. 2.7, only the left-hand poles, the right-hand poles have been discarded, the poles are $p_{1}=-0.2588+\mathrm{j} 0.9659, p_{2}=-0.7071-\mathrm{j} 0.7071, p_{3}=-0.9959+\mathrm{j} 0.2588, p_{4}=$ $-0.9959-\mathrm{j} 0.2588, p_{5}=-0.7071+\mathrm{j} 0.7071, p_{6}=-0.2588-\mathrm{j} 0.9659$.

Since $D(p)$ should be all real and bigger than zero, so that the circuit could be realized. Also, its all-roots should lie on the left-half plane. $D(p)$ is given by

$$
D(p)=\prod_{r=1}^{N}\left(p-\mathrm{j} \exp \left(\mathrm{j} \theta_{r}\right)\right)
$$

Finally

$$
S_{11}(p)=\frac{ \pm p^{N}}{\prod_{r=1}^{N}\left(p-\mathrm{j} \exp \left(\mathrm{j} \theta_{r}\right)\right)}
$$

Thus, for a butterworth of second order

$$
S_{11}(p)=\frac{ \pm p^{2}}{\left(p-p_{1}\right)\left(p-p_{2}\right)}=\frac{ \pm p^{2}}{p^{2}+\sqrt{2} p+1}
$$

Now

$$
Z(p)=\frac{1+\sqrt{2} p+2 p^{2}}{1+\sqrt{2} p}=\sqrt{2} p+\frac{1}{\sqrt{2} p+1}
$$

or

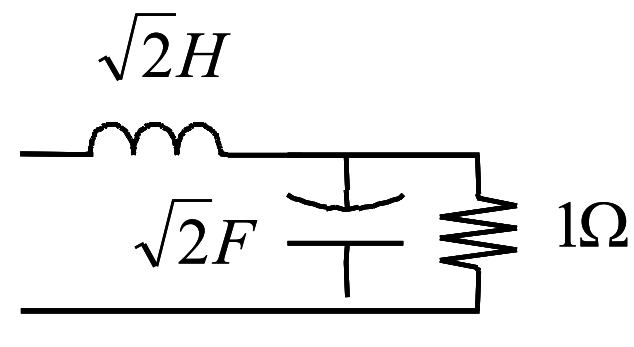

(a)

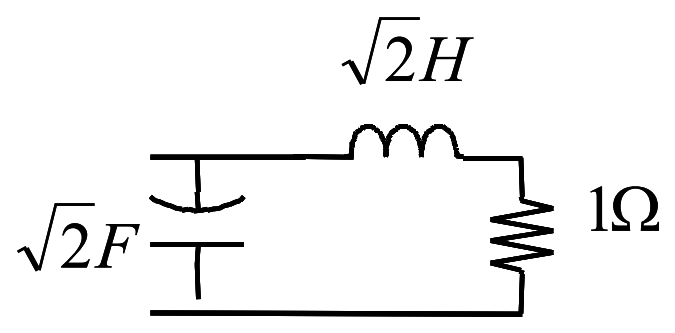

(b)

Figure 2.8.: Realization techniques. 


$$
Y(p)=\frac{1+\sqrt{2} p+2 p^{2}}{1+\sqrt{2} p}=\sqrt{2} p+\frac{1}{\sqrt{2} p+1}
$$

giving the two realizations shown in Fig. 2.8a, and Fig. 2.8b. Fig. 2.9 shows the Butterworth response for $n=1,2,4,6$. For Butterworth, a closed form equations can be obtained to calculate

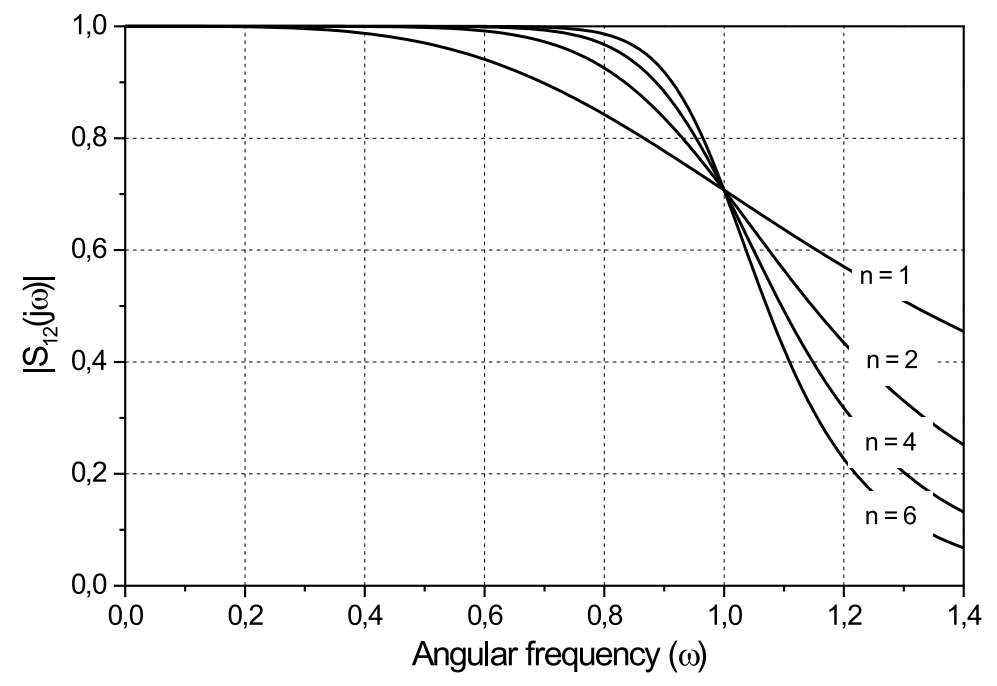

Figure 2.9.: Butterworth lowpass characteristic.

the lowpass ladder circuit elements. Assume the two-element low-pass filter prototype shown in Fig. 2.10. Assuming also a source impedance of $1 \Omega$. The input impedance of this filter is

$$
Z_{i n}(\mathrm{j} \omega)=\mathrm{j} \omega L+\frac{R(1-\mathrm{j} \omega R C)}{1+\omega^{2} R^{2} C^{2}}
$$

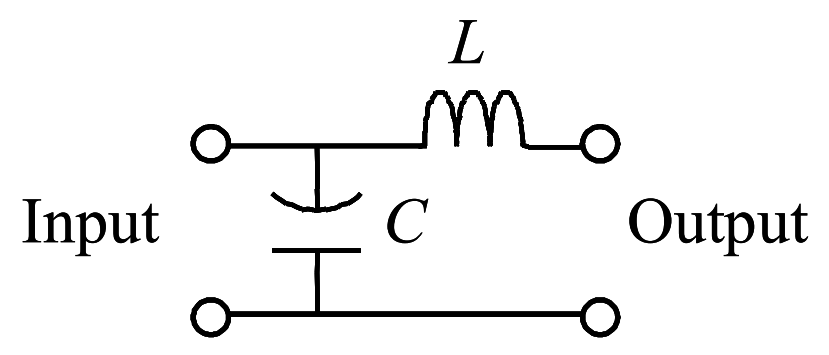

Figure 2.10.: Two-element lowpass prototype.

The power loss of a second-order will be

$$
L_{A}=10 \log _{10}\left(1+\omega^{4}\right) d B
$$


Thus the power loss ratio is defined as

$$
\begin{aligned}
P_{L R} & =\frac{1}{1-\left|S_{11}(\mathrm{j} \omega)\right|^{2}} \\
& =1+\omega^{4}
\end{aligned}
$$

Thus

$$
\begin{gathered}
P_{L R}=\frac{1}{1-\left(\left(Z_{i n}(\mathrm{j} \omega)-1\right) /\left(Z_{i n}(\mathrm{j} \omega)+1\right)\right)\left(\left(Z_{i n}^{*}(\mathrm{j} \omega)-1\right) /\left(Z_{i n}^{*}(\mathrm{j} \omega)+1\right)\right)} \\
=\frac{\left|Z_{i n}(\mathrm{j} \omega)+1\right|^{2}}{2\left(Z_{i n}(\mathrm{j} \omega)+Z_{i n}^{*}(\mathrm{j} \omega)\right)} \\
=\frac{1+\omega^{2} R^{2} C^{2}}{4 R}\left[\left[\frac{R}{1+\omega^{2} R^{2} C^{2}}+1\right]^{2}+\left[\omega L-\frac{\omega C R^{2}}{\omega^{2} R^{2} C^{2}}\right]^{2}\right] \\
=\frac{1}{4 R}\left[R^{2}+2 R+1+\omega^{2} R^{2} C^{2}+\omega^{2} L^{2}+\omega^{4} R^{2} L^{2} C^{2}-2 \omega^{2} L C R^{2}\right] \\
=\frac{1}{4 R}\left[(1-R)^{2}+\left(R^{2} C^{2}+L^{2}-2 L C R^{2}\right) \omega^{2}+\omega^{4} R^{2} L^{2} C^{2}\right] \\
=1+\omega^{4}
\end{gathered}
$$

Since $P_{L R}=1$ for $\omega=0$ and $R=1$. In addition the coefficient of $\omega^{2}$ must vanish, so

$$
C^{2}+L^{2}-2 L C=(C-L)^{2}=0
$$

or $L=C$. Then for the coefficient of $\omega^{4}$ to be unity, thus

$$
\frac{1}{4} C^{2} L^{2}=\frac{1}{4} L^{4}=1
$$

thus

$$
L=C=\sqrt{2}
$$


This procedure can be extended to find the element values for filters with an arbitrary number of elements, $N$. The Butterworth prototype values for $N$ ladder network order can be calculated from the equations below [58]

$$
\begin{aligned}
g_{0} & =1 \\
g_{r} & =2 \sin \left[\frac{(2 r-1) \pi}{2 N}\right] \quad r=1,2, \ldots N \\
g_{N+1} & =1 \text { for all } N
\end{aligned}
$$

These elements often expressed by tables, like in Table 2.1. Fig. 2.11 shows a filter having three reactive elements, this elements alternate between series and shunt connections.

Table 2.1.: Element values for the Butterworth Ladder Filter with $g_{0}=1, \omega_{c}=1$, and $\mathrm{n}=1$ to 9 .

\begin{tabular}{ccccccccccc}
\hline $\mathrm{n}$ & $g_{1}$ & $g_{2}$ & $g_{3}$ & $g_{4}$ & $g_{5}$ & $g_{6}$ & $g_{7}$ & $g_{8}$ & $g_{9}$ & $g_{10}$ \\
\hline 1 & 2.0000 & 1.0 & & & & & & & & \\
2 & 1.4142 & 1.4142 & 1.0 & & & & & & & \\
3 & 1.0000 & 2.0000 & 1.0000 & 1.0 & & & & & & \\
4 & 0.7654 & 1.8478 & 1.8478 & 0.7654 & 1.0 & & & & & \\
5 & 0.6180 & 1.6180 & 2.0000 & 1.6180 & 0.6180 & 1.0 & & & & \\
6 & 0.5176 & 1.4142 & 1.9318 & 1.9318 & 1.4142 & 0.5176 & 1.0 & & & \\
7 & 0.4450 & 1.2470 & 1.8019 & 2.0000 & 1.8019 & 1.2470 & 0.4450 & 1.0 & & \\
8 & 0.3902 & 1.1111 & 1.6629 & 1.9616 & 1.9616 & 1.6629 & 1.1111 & 0.3902 & 1.0 & \\
9 & 0.3473 & 1.0000 & 1.5321 & 1.8794 & 2.0000 & 1.8794 & 1.5321 & 1.0000 & 0.3473 & 1.0 \\
\hline
\end{tabular}

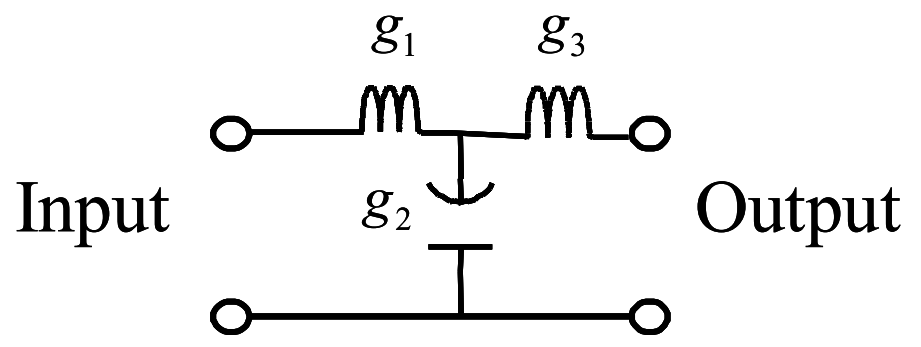

Figure 2.11.: Ladder circuit for lowpass filter prototype of three order and their element definitions.

\subsubsection{The Chebyshev Prototype}

The Chebyshev filter is also known as equal-ripple filter due to the occurrence of ripples in the passband. It is based on Chebyshev polynomials:

$$
T_{N}(\omega)=\cos \left[N \cos ^{-1}(\omega)\right]
$$


Table 2.2.: Chebyshev first kind polynomials

\begin{tabular}{cc}
\hline $\mathrm{n}$ & $T_{n}(\omega)$ \\
\hline 0 & 1 \\
1 & $\omega$ \\
2 & $2 \omega^{2}-1$ \\
3 & $4 \omega^{3}-3 \omega$ \\
4 & $8 \omega^{4}-8 \omega^{2}+1$ \\
5 & $16 \omega^{5}-20 \omega^{3}+5 \omega$ \\
6 & $32 \omega^{6}-48 \omega^{4}+18 \omega^{2}-1$ \\
\hline
\end{tabular}

where $N$ is the order of the filter. The explicit form of the Chebyshev polynomials of the first kind and degrees 1 to 6 is given in Table. 2.2. Fig. 2.12 shows plots of the Chebyshev polynomials $T_{N}(\omega)$ of degrees 1 to 5 .

Chebyshev is an amplitude filter carried out via recursion (rather than convolution). It achieves a fast roll off by allowing an equiripple in the frequency response - the more ripple, the faster the roll off. It has a non linear phase response. The magnitude response of $N$ order Chebyshev filter is given by

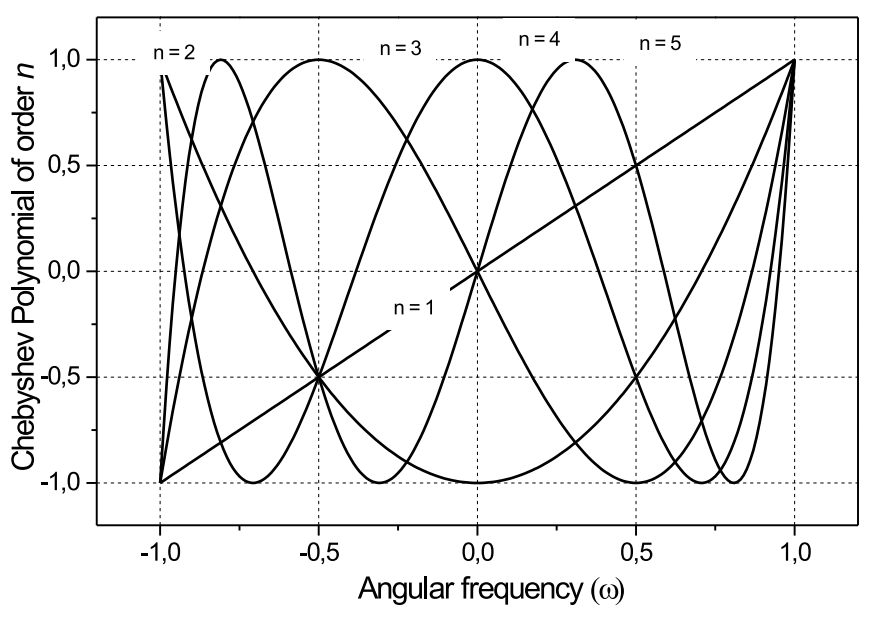

Figure 2.12.: Chebyshev polynomials curves.

$$
|H(\mathrm{j} \omega)|=\frac{1}{\sqrt{1+\xi^{2} T_{N}^{2}(\omega)}}
$$

The insertion loss at ripple level is normally expressed as

$$
L_{A}=10 \log _{10}\left(1+\xi^{2} T_{N}^{2}(\omega)\right) d B
$$


where $\xi$ is the passband level. Fig. 2.13 shows the plots of the Chebyshev lowpass for $\xi=0.25$ and $n=4,6,8$. Now, the synthesis of the Chebyshev filter proceed as follows:

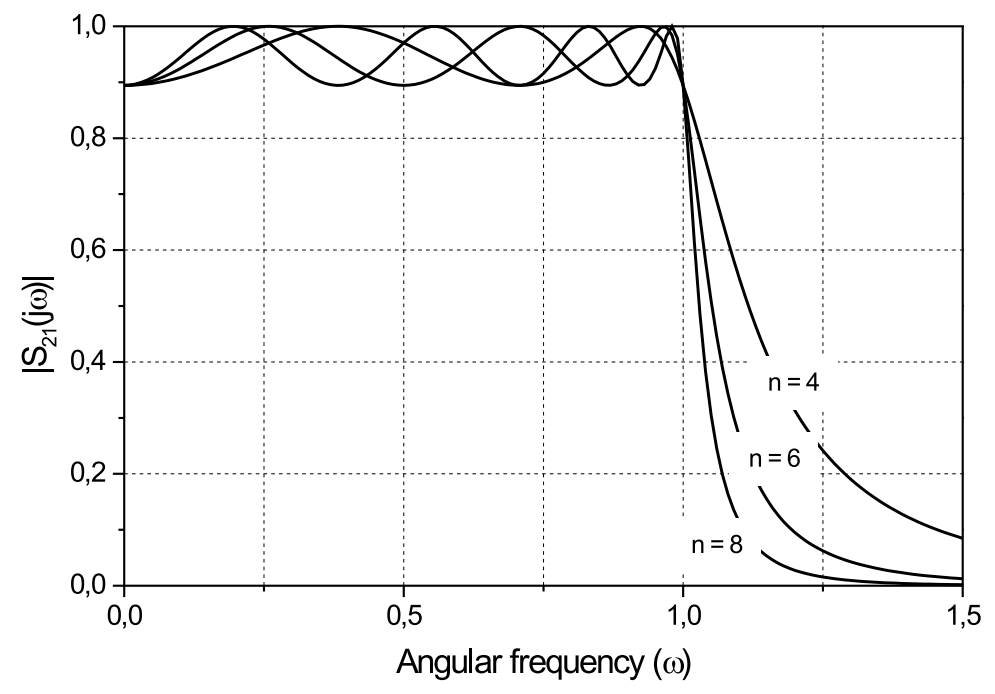

Figure 2.13.: Chebyshev lowpass characteristic.

$$
\left|S_{12}(\mathrm{j} \omega)\right|^{2}=\frac{1}{1+\xi^{2} T_{N}^{2}(\omega)}
$$

To determine the transfer function by factorization, again this requires the computation of the poles of (2.9). Thus, the poles are at those values of $p$ for which

$$
\left[T_{N}^{2}(p)\right]_{p=\mathrm{j} \omega}=\frac{-1}{\xi^{2}}
$$

and since

$$
T_{N}(p)=\cos \left[N \cos ^{-1}(p)\right]
$$

That is ,

$$
\cos ^{2}\left[N \cos ^{-1}(p)\right]+\frac{1}{\xi^{2}}=0
$$

By solving for $p$, yields

$$
\cos ^{-1}(\mathrm{j} p)=\sin ^{-1}(\mathrm{j} \psi)+\theta_{r}
$$

where

$$
\psi=\sinh \left[\frac{1}{N} \sinh ^{-1}\left(\frac{1}{\xi}\right)\right]
$$


and

$$
\theta_{r}=\frac{(2 r-1) \pi}{2 N}
$$

Thus

$$
p_{r}=\mathrm{j} \cos \left[\sin ^{-1}(\mathrm{j} \psi)+\theta_{r}\right]
$$

The left half-plane poles occurs for $r=1 \ldots N$.

$$
p_{r}=-\psi \sin \left(\theta_{r}\right)-\mathrm{j}\left(1-\psi^{2}\right)^{1 / 2} \cos \left(\theta_{r}\right), \quad \text { for } r=1 \quad \ldots \quad 2 N
$$

Now

$$
\begin{aligned}
p_{r} & =\sigma_{r}+\mathrm{j} \omega_{r} \\
& =-\psi \sin \left(\theta_{r}\right)-\mathrm{j}\left(1-\psi^{2}\right)^{1 / 2} \cos \left(\theta_{r}\right), \quad \text { for } r=1 \quad \ldots \quad 2 N
\end{aligned}
$$

Thus,

$$
\begin{aligned}
\sigma_{r} & =-\psi \sin \left(\theta_{r}\right) \\
\omega_{r} & =-\left(1-\psi^{2}\right)^{1 / 2} \cos \left(\theta_{r}\right)
\end{aligned}
$$

Hence

$$
\begin{aligned}
\frac{-\sigma_{r}}{\psi} & =\sin \left(\theta_{r}\right) \\
\frac{-\omega_{r}}{\left(1-\psi^{2}\right)^{1 / 2}} & =\cos \left(\theta_{r}\right)
\end{aligned}
$$

Now since

$$
\cos ^{2}\left(\theta_{r}\right)+\sin ^{2}\left(\theta_{r}\right)=1
$$

Therefore

$$
\left(\frac{\sigma_{r}}{\psi}\right)^{2}+\left(\frac{-\omega_{r}}{\left(1-\psi^{2}\right)^{1 / 2}}\right)^{2}=1
$$


That is;

$$
\frac{\sigma_{r}^{2}}{\psi^{2}}+\frac{\omega_{r}^{2}}{1-\psi^{2}}=1
$$

Thus, the poles of the lowpass prototype utilizes a Chebyshev functions lie on an ellipse. Fig. 2.14(a) shows the poles of Chebyshev second order in the $\sigma-\omega$ plane. Fig. 2.14(b) shows a 3D plot for these poles, where the poles given by: for $\xi=0.25, p_{1}=-0.8836+\mathrm{j} 0.3971$, $p_{2}=-0.8836-\mathrm{j} 0.3971,-p_{1}^{*}=+0.8836+\mathrm{j} 0.3971,-p_{2}^{*}=+0.8836+\mathrm{j} 0.3971$, for $\xi=0.5$ the poles will locate as follows. $p_{1}=-0.5559+\mathrm{j} 0.2701, p_{2}=-0.5559-\mathrm{j} 0.2701,-p_{1}^{*}=+0.5559+\mathrm{j} 0.2701$, $-p_{2}^{*}=+0.5559+$ j0.2701. Fig. 2.15 shows the Chebyshev $6^{\text {th }}$ order lowpass filter poles imposed with that one for Butterworth $6^{\text {th }}$ order.

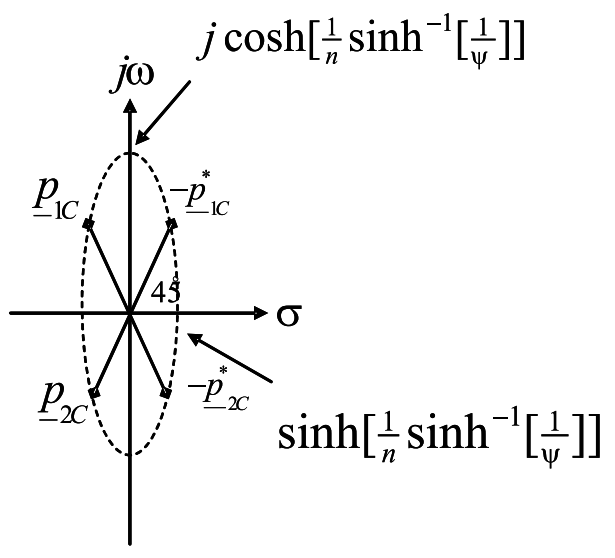

(a) projection on $\sigma-\omega$ plane.

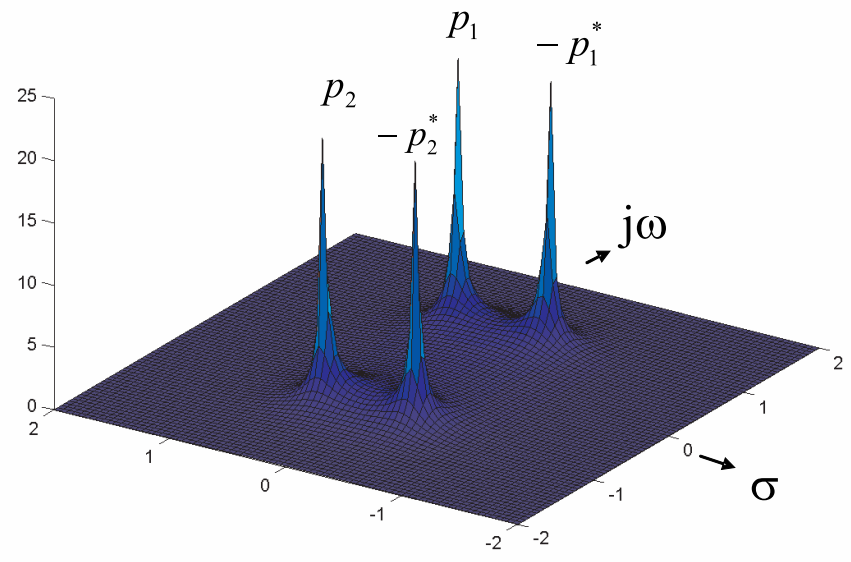

(b) 3D view for the Poles.

Figure 2.14.: Poles of the second order Chebyshev transfer function

Now, from the unitary condition

$$
\left|S_{11}(\mathrm{j} \omega)\right|^{2}=1-\left|S_{12}(\mathrm{j} \omega)\right|^{2}=\frac{\xi^{2} T_{N}^{2}(\omega)}{1+\xi^{2} T_{N}^{2}(\omega)}
$$

It may be shown that [59]

$$
S_{11}(p)=\prod_{r=1}^{N}\left\{\frac{p+\mathrm{j} \cos \left(\theta_{r}\right)}{p+\mathrm{j} \cos \left[\sin ^{-1}(\mathrm{j} \psi)+\theta_{r}\right]}\right\}
$$




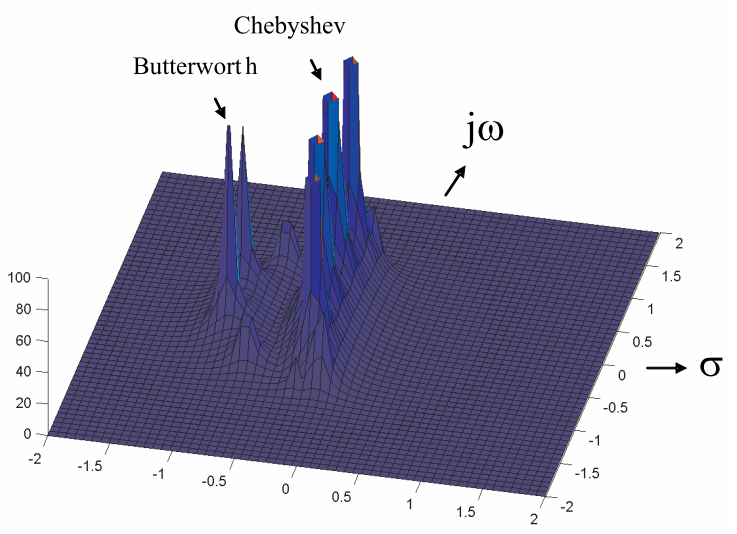

Figure 2.15.: Poles of the $6^{\text {th }}$ order Chebyshev imposed with Butterworth of the same order.

The network can then be synthesized as a lowpass filter ladder network by formulation of $Z(p)$. The Chebyshev prototype values for $N$ ladder network order can be calculated from the equations below [58]

$$
\begin{gathered}
g_{1}=\frac{2 a_{1}}{\sinh \left[\frac{\phi}{2 n}\right]} \\
g_{k}=\frac{4 a_{k} a_{k-1}}{d_{k-1} g_{k-1}} \text { for } k=2,3, \quad \ldots \quad n \\
g_{n+1}=1 \quad \text { for } n \text { odd } \\
g_{n+1}=\operatorname{coth}^{2}(\phi / 4) \text { for } n \text { even }
\end{gathered}
$$

where

$$
\begin{gathered}
a_{k}=\sin \left[\frac{(2 k-1) \pi}{2 n}\right] \text { for } k=1,2,3, \ldots \quad n \\
d_{k}=\left[\sinh ^{2}\left(\frac{\phi}{2 n}\right)+\sin ^{2}\left(\frac{k \pi}{n}\right)\right] \text { for } k=1,2,3, \ldots \quad n \\
\phi=\ln \left[\operatorname{coth}\left(\frac{\xi}{17.37}\right)\right]
\end{gathered}
$$

Like in Butterworth, these elements often expressed by tables, like in Tables 2.3 and 2.4. 
Table 2.3.: Element values for the Chebyshev Ladder Filter with $0.01 \mathrm{~dB}$ ripple with $g_{0}=1, \omega_{c}=1$, and $\mathrm{n}=1$ to 9 .

\begin{tabular}{ccccccccccc}
\hline $\mathrm{n}$ & $g_{1}$ & $g_{2}$ & $g_{3}$ & $g_{4}$ & $g_{5}$ & $g_{6}$ & $g_{7}$ & $g_{8}$ & $g_{9}$ & $g_{10}$ \\
\hline 1 & 0.0960 & 1.0 & & & & & & & & \\
2 & 0.4489 & 0.4078 & 1.1008 & & & & & & & \\
3 & 0.6292 & 0.9703 & 0.6292 & 1.0 & & & & & & \\
4 & 0.7129 & 1.2004 & 1.3213 & 0.6476 & 1.1008 & & & & & \\
5 & 0.7563 & 1.3049 & 1.5773 & 1.3049 & 0.7563 & 1.0 & & & & \\
6 & 0.7814 & 1.3600 & 1.6897 & 1.5350 & 1.4970 & 0.7098 & 1.1008 & & & \\
7 & 0.7970 & 1.3924 & 1.7481 & 1.6331 & 1.7481 & 1.3924 & 0.7970 & 1.0 & & \\
8 & 0.8073 & 1.4131 & 1.7825 & 1.6833 & 1.8529 & 1.6193 & 1.5555 & 0.7334 & 1.1008 & \\
9 & 0.8145 & 1.4271 & 1.8044 & 1.7125 & 1.9058 & 1.7125 & 1.8044 & 1.4271 & 0.8145 & 1.0 \\
\hline
\end{tabular}

Table 2.4.: Element values for the Chebyshev Ladder Filter with $0.1 \mathrm{~dB}$ ripple with $g_{0}=1, \omega_{c}=1$, and $\mathrm{n}=1$ to 9 .

\begin{tabular}{ccccccccccc}
\hline $\mathrm{n}$ & $g_{1}$ & $g_{2}$ & $g_{3}$ & $g_{4}$ & $g_{5}$ & $g_{6}$ & $g_{7}$ & $g_{8}$ & $g_{9}$ & $g_{10}$ \\
\hline 1 & 0.3052 & 1.0 & & & & & & & & \\
2 & 0.8431 & 0.6220 & 1.3554 & & & & & & & \\
3 & 1.0316 & 1.1474 & 1.0316 & 1.0 & & & & & & \\
4 & 1.1088 & 1.3062 & 1.7704 & 0.8181 & 1.3554 & & & & & \\
5 & 1.1468 & 1.3712 & 1.9750 & 1.3712 & 1.1468 & 1.0 & & & & \\
6 & 1.1681 & 1.4040 & 2.0562 & 1.5171 & 1.9029 & 0.8618 & 1.3554 & & & \\
7 & 1.1812 & 1.4228 & 2.0967 & 1.5734 & 2.0967 & 1.4228 & 1.1812 & 1.0 & & \\
8 & 1.1898 & 1.4346 & 2.1199 & 1.6010 & 2.1700 & 1.5641 & 1.9445 & 0.8778 & 1.3554 & \\
9 & 1.1957 & 1.4426 & 2.1346 & 1.6167 & 2.2054 & 1.6167 & 2.1346 & 1.4426 & 1.1957 & 1.0 \\
\hline
\end{tabular}




\subsubsection{Bessel Filters}

The previous filter types specify the amplitude response, but in some applications (such as multiplexing filters) it is important to have highly linear phase filter response with constant group delay across the entire passband. Bessel filters based on Bessel functions are used for such applications and they are show relatively slow attenuation in the transition-band without ripples.

\subsubsection{Impedance scaling and Frequency Transformation}

To convert from a $1 \Omega$ impedance level to an impedance level of $Z_{0} \Omega$, the impedances of the all circuit elements in the filter are scaled, the inductor $L$ becomes $Z_{0} L$ and the capacitor $C$ becomes $C / Z_{0}$. Also the cutoff frequency of the normalized filter convert to an arbitrary frequency $\omega_{c}$ using the following transformation

$$
\omega \rightarrow \frac{\omega}{\omega_{c}}
$$

Applying this transformation and scaling to the inductors and the capacitors yields

$$
\begin{aligned}
L_{k} & \rightarrow \frac{Z_{0} L_{k}}{\omega_{c}} \\
C_{k} & \rightarrow \frac{C_{k}}{Z_{0} \omega_{c}}
\end{aligned}
$$

\subsubsection{Poles Pattern of a Lowpass Filter}

The poles of the lowpass filter is also subject to the frequency transformation and the scaling has no influence on them. Therefore

$$
s^{\prime} \rightarrow \frac{s}{\omega_{c}}
$$

where $s$ is the laplace variable.

The second order Butterworth lowpass filter with cutoff frequency $1 \mathrm{~Hz}$ is

$$
H_{L P P}(s)=\frac{1}{s^{2}+\sqrt{2} s+1}
$$

Hence, a second order lowpass filter with cutoff frequency $\omega_{c}$ can be designed:

$$
H_{L P}(s)=H_{L P P}\left(s^{\prime}\right)=\frac{1}{\left[\frac{s}{\omega_{c}}\right]^{2}+\sqrt{2}\left[\frac{s}{\omega_{c}}\right]+1}
$$


To find the poles for this filter the denominator is set to be zero,

$$
\left[\frac{s}{\omega_{c}}\right]^{2}+\sqrt{2}\left[\frac{s}{\omega_{c}}\right]+1=0
$$

by solving for $s$ in terms of $\omega_{c}$ this yields two poles given by

$$
\begin{aligned}
& p_{1_{c}}=-\omega_{c} \frac{1}{\sqrt{2}}[1+\mathrm{j}] \\
& p_{2_{c}}=-\omega_{c} \frac{1}{\sqrt{2}}[1-\mathrm{j}]
\end{aligned}
$$

Fig. 2.16 shows the poles pattern of a second-order lowpass filter after and before the frequency transformation. The poles of the normalized prototype are all locate on a unit circle, while for the transformed filter lie on a circle of a radius $\omega_{c}$. Fig. 2.17 shows a 3D view.

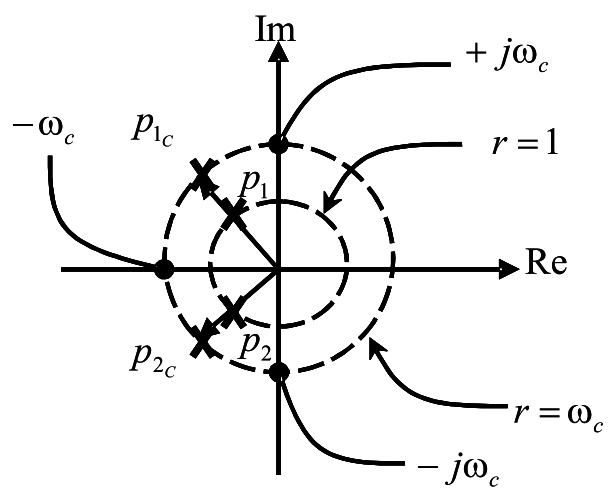

Figure 2.16.: Transformed poles of the low prototype to $\omega_{c}$.

\subsection{Bandpass Filter Design}

To convert the lowpass prototype into a bandpass filter with arbitrary center frequency and bandwidth the band-edges for the lowpass prototype at $\omega= \pm 1$ are mapped into the band-edges of the bandpass filter at $\omega_{1}, \omega_{2}$. Also the midband of the lowpass prototype at $\omega=0$ mapped into the center frequency of the passband of the bandpass filter.

Thus the following transformation is used

$$
\omega \rightarrow \alpha\left[\frac{\omega}{\omega_{0}}-\frac{\omega_{0}}{\omega}\right]
$$

For $\omega=-1$ and $\omega=+1$ to map to $\omega_{1}, \omega_{2}$ then 


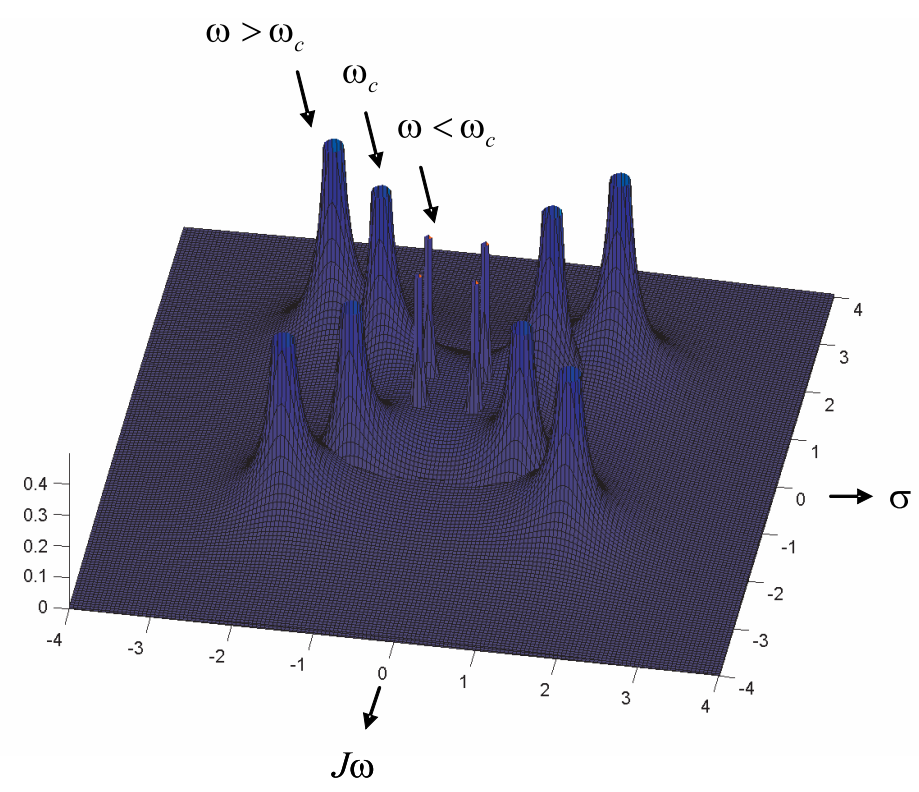

Figure 2.17.: Transformed poles of the low prototype to $\omega_{c}=1.5, \omega<\omega_{c}=0.5, \omega>\omega_{c}=2.5 \mathrm{rads}^{-1}$.

$$
\begin{aligned}
& -1 \rightarrow \alpha\left[\frac{\omega_{1}}{\omega_{0}}-\frac{\omega_{0}}{\omega_{1}}\right] \\
& +1 \rightarrow \alpha\left[\frac{\omega_{2}}{\omega_{0}}-\frac{\omega_{0}}{\omega_{2}}\right]
\end{aligned}
$$

solving the two previous equations simultaneously yields

$$
\omega=\left(\omega_{1} \omega_{2}\right)^{1 / 2}
$$

and

$$
\alpha=\frac{\omega_{0}}{\omega_{2}-\omega_{1}}
$$

Now, applying this transformation to a series inductor of the lowpass prototype, yields

$$
Z=\mathrm{j} \omega L \rightarrow \mathrm{j} L \alpha\left[\frac{\omega}{\omega_{0}}-\frac{\omega_{0}}{\omega}\right]=\mathrm{j}\left[\frac{\alpha L}{\omega_{0}}\right] \omega-\frac{\mathrm{j}}{\omega\left[1 / \alpha L \omega_{0}\right]}
$$

Thus the series inductor of the lowpass prototype is converted to a series $L C$ circuit on the bandpass circuit. Similar to the shunt capacitor

$$
Y=\mathrm{j} \omega C \rightarrow \mathrm{j} C \alpha\left[\frac{\omega}{\omega_{0}}-\frac{\omega_{0}}{\omega}\right]=\mathrm{j}\left[\frac{\alpha C}{\omega_{0}}\right] \omega-\frac{\mathrm{j}}{\omega\left[1 / \alpha C \omega_{0}\right]}
$$


Thus, the shunt capacitor of the lowpass prototype is converted to a shunt $L C$ circuit on the bandpass filter. Thus, the lowpass filter elements are converted to series resonant circuits (low impedance at resonance) in the series arms, and to parallel resonant circuits (high impedance at resonance) in the shunt arms. Both the series and the parallel resonant elements have a resonant frequency of $\omega_{0}$.

Fig. 2.18 shows the resulted bandpass circuit from the transformed lowpass prototype circuit of Fig. 2.11, where the two series elements $g_{1}$ and $g_{3}$ are transformed to a series $L_{1} C_{1}, L_{3} C_{3}$ respectively. $g_{2}$ is transformed to a shunt $L_{2} C_{2}$.

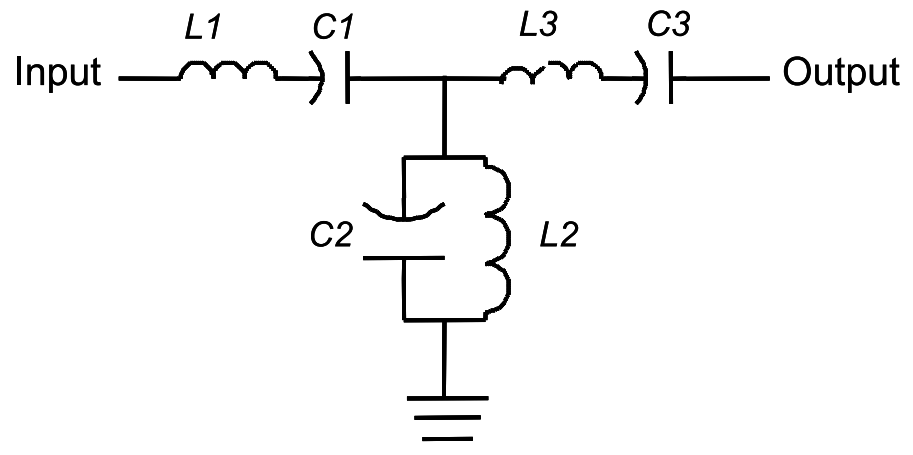

Figure 2.18.: Bandpass filter circuit resulted from the transformation of the lowpass prototype.

Fig. 2.19 shows the bandpass filter characteristics that have been transformed from the lowpass prototype, Fig. 2.19(b) resulted from Butterworth prototype and Fig. 2.19(b) from Chebyshev. The three previously mentioned types of filter with the same order have been designed. Their elements values are shown in Table (4.4). The amplitude results for these three filters are shown in Fig. 2.20.

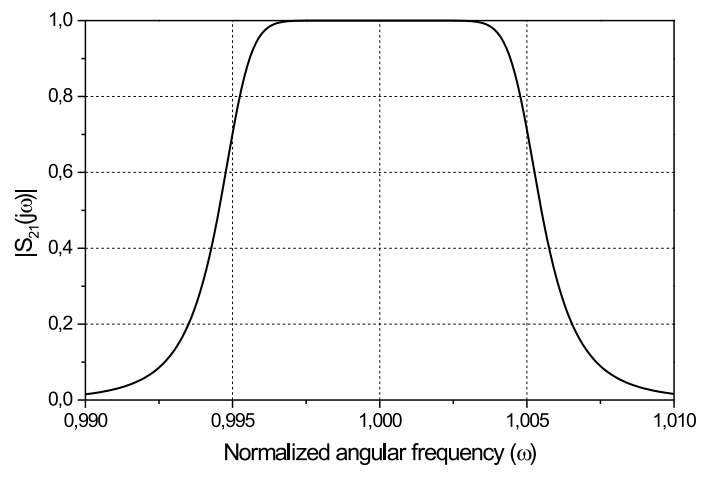

(a) Butterworth bandpass.

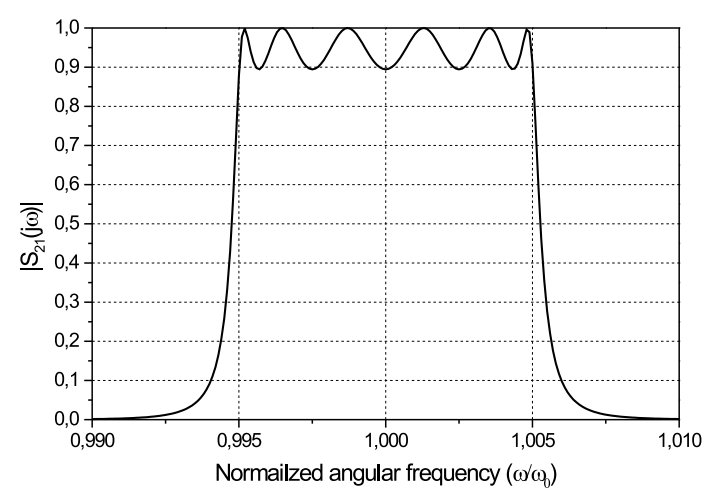

(b) Chebyshev bandpass.

Figure 2.19.: Catalog of bandpass filter types

The major differences in output response: Chebyshev rolls off faster than the butterworth filter. The equal ripple response has the sharpest cutoff. The maximally flat response has a filter flatter attenuation characteristics in the passband, but slightly lower cutoff rate. A Chebyshev filter with a ripple of $0.5 \%$ has an almost flat passband but has a much better 
Table 2.5.: Elements values for a bandpass filter for various types.

\begin{tabular}{lllllll}
\hline & $C_{1}$ & $L_{1}$ & $C_{2}$ & $L_{2}$ & $C_{3}$ & $L_{3}$ \\
$(\mathrm{pF})$ & $(\mathrm{nH})$ & $(\mathrm{pF})$ & $(\mathrm{nH})$ & $(\mathrm{pF})$ & $(\mathrm{nH})$ \\
\hline Ripple & 0.199 & 127 & 34.91 & 0.726 & 0.199 & 127 \\
Flat & 0.318 & 79.57 & 63.6 & 0.397 & 0.318 & 79.57 \\
Phase & 0.7354 & 34.44 & 33 & 0.763 & 0.1412 & 179.38 \\
\hline
\end{tabular}

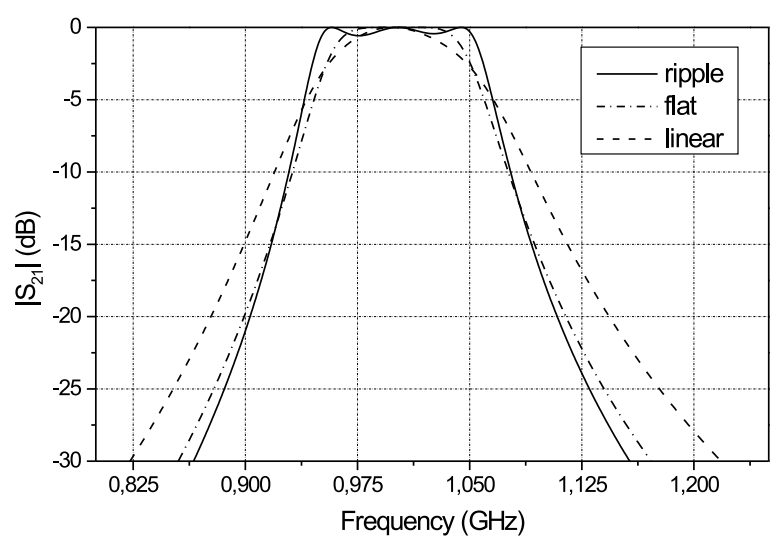

Figure 2.20.: Amplitude response for the three bandpass filter types.

roll off when compared to a butterworth filter. Chebyshev has ripples in either the pass or stop band while Butterworth is monotonic. Butterworth rolls off faster than the Bessel filter. Finally, The linear phase filter has the worst cutoff rate.

\subsubsection{Poles Pattern in the Bandpass filter}

As in the transformed lowpass, the poles of the lowpass prototype are transform to that one for the bandpass filter as follows:

$$
s^{\prime} \rightarrow \frac{s^{2}+\omega_{1} \omega_{2}}{s\left[\omega_{2}-\omega_{1}\right]}
$$

The above equation can be written as follows

$$
s^{\prime} \rightarrow \frac{s^{2}+\omega_{0}^{2}}{s \Delta \omega}
$$

where $\Delta \omega$ is the filter passband bandwidth. The second order Butterworth lowpass filter with cutoff frequency $1 \mathrm{~Hz}$ is

$$
H_{L L P}(s)=\frac{1}{s^{2}+\sqrt{2} s+1}
$$


Let $s=p$ be a pole of the lowpass prototype. Then

$$
H_{B P}(s)=H_{L L P}\left(s^{\prime}\right)=\frac{1}{\left[\frac{s^{2}+\omega_{0}^{2}}{s \Delta \omega}\right]^{2}+\sqrt{2}\left[\frac{s^{2}+\omega_{0}^{2}}{s \Delta \omega}\right]+1}
$$

To find the poles for this filter the denominator is set to be zero,

$$
\left[\frac{s^{2}+\omega_{0}^{2}}{s \Delta \omega}\right]^{2}+\sqrt{2}\left[\frac{s^{2}+\omega_{0}^{2}}{s \Delta \omega}\right]+1=0
$$

poles at

$$
p_{b}=\frac{p \Delta \omega}{2} \pm \sqrt{\left(\frac{p \Delta \omega}{2}\right)^{2}-\omega_{0}^{2}}=\frac{p \Delta \omega}{2} \pm j \omega_{0} \sqrt{1-\left(\frac{p \Delta \omega}{2 \omega_{0}}\right)^{2}}
$$

if $\omega_{0}>>|\Delta \omega|$, as is often the case (narrow-band filter), poles are at

$$
p_{b} \simeq \frac{p \Delta \omega}{2} \pm \mathrm{j} \omega_{0}
$$

Therefore

$$
p_{b}=\frac{-\sin \left(\theta_{r}\right)+\mathrm{j} \cos \left(\theta_{r}\right) \Delta \omega}{2} \pm \mathrm{j} \omega_{0} \quad r=1, \ldots, 2 N
$$

Therefore

$$
p_{b}=-\frac{\Delta \omega}{2} \sin \left(\theta_{r}\right)+\mathrm{j}\left(\frac{\Delta \omega}{2} \cos \left(\theta_{r}\right) \pm \omega_{0}\right) \quad r=1, \ldots, 2 N
$$

i.e. the pole pattern is reproduced at half-scale of the bandwidth, but with the origin transferred to $\pm j \omega_{0}$. Fig. 2.21 shows the poles pattern for the bandpass filter. The poles of the lowpass $p_{1}, p_{2}$, the center of the unit circle has been transformed to $\pm \mathrm{j} \omega_{0}$, while the radius of the unit circuit has been scaled by $\Delta \omega / 2$. Fig. 2.22 shows the corresponding $3 \mathrm{D}$ view. The poles are given as: $p_{1 B}=-0.0353+\mathrm{j} 1.7854, p_{2 B}=-0.0353+\mathrm{j} 1.7147, p_{3 B}=-0.0353-\mathrm{j} 1.7147$, $p_{4 B}=-0.0353-\mathrm{j} 1.7854$. Finally, $p_{1 L}=-0.707+\mathrm{j} 0.707, p_{1 L}=-0.707-\mathrm{j} 0.707$. 


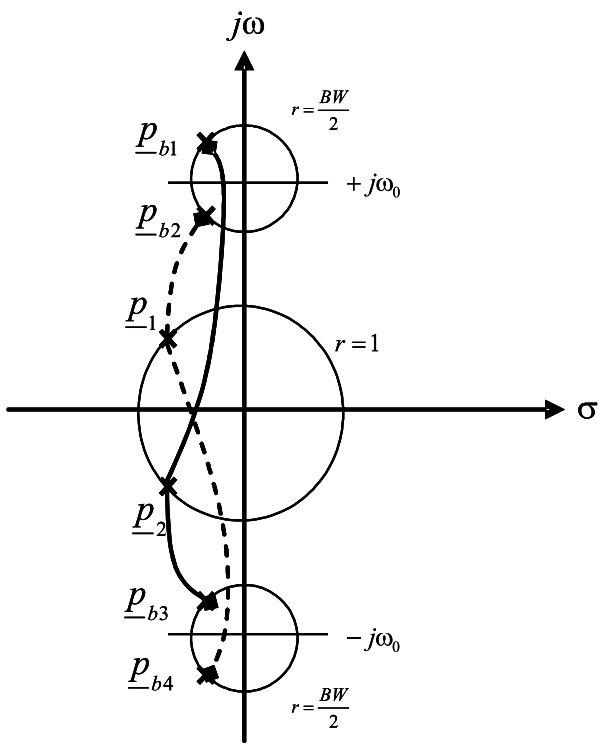

Figure 2.21.: Bandpass filter poles pattern.

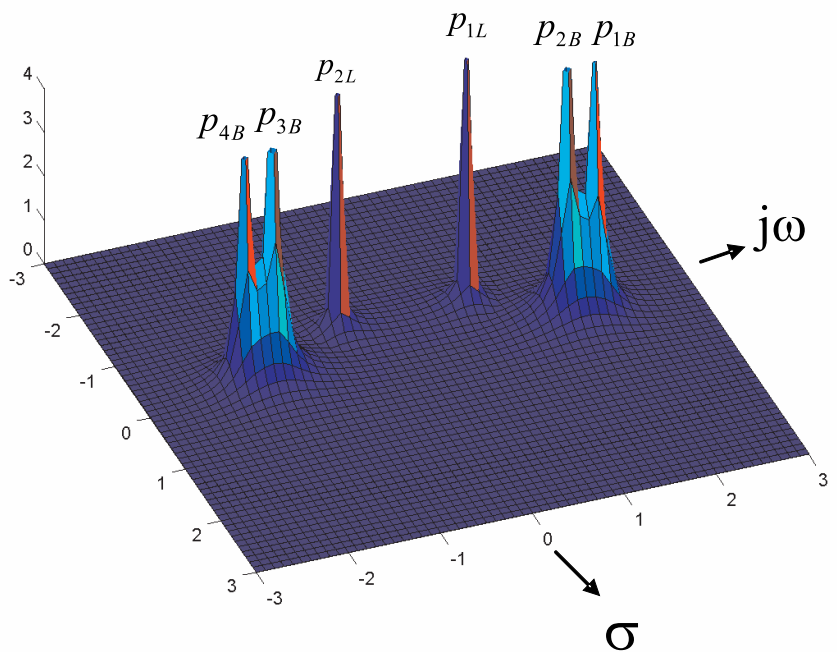

Figure 2.22.: The transformed poles pattern from lowpass to bandpass filter at $\omega_{0}=1.75$ and $\Delta \omega=0.1$. 


\subsubsection{Filters with Distributed Elements}

The lumped-element filter design generally worked well at low frequencies, but two problems arise at microwave frequencies [60]: first lumped elements such as inductors and capacitors are generally available for a limited range of values and are difficult to implement at microwave frequencies. Secondly, at microwave frequencies the distance between filter components is not negligible. For microwave applications such design should be modified to use distributed elements consisting of transmission line sections [61].

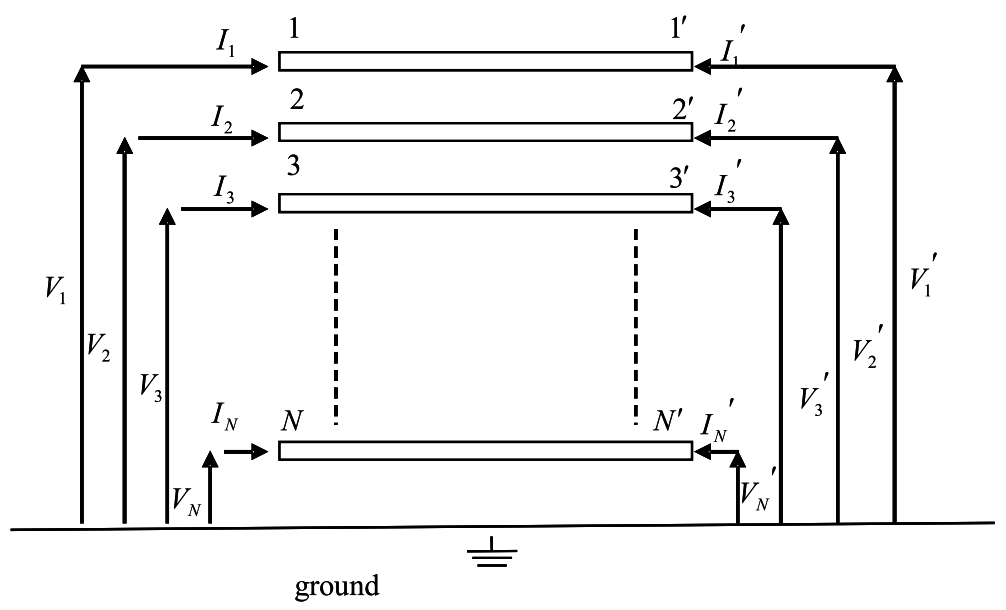

Figure 2.23.: A symmetrical coupled-line N-port.

The distributed lines can be realized in any desired form such as waveguide, coaxial lines, or planar transmission lines. Kuroda's identities [62], allow one to realize low-pass structures using shunt elements with identical response called unit elements and with Richards transformations [63] one can establish the distributed line parameters. The parallel-coupled transmission lines can be used to construct many types of filters. The design of parallel-coupled resonators line filters was formulated in [64]. Terminal characteristic parameters for a uniform coupled-line $N$-port for the general case of a symmetric, inhomogeneous system, as shown in Fig. 2.23, are derived in [65]. A two-port network can be formed from the coupled line section by terminating the ports in either open or short circuits.

Assuming only a TEM mode (microstrip lines topology) of propagation and assigning the currents and voltages to each line. The admittance matrix can be described by [66]

$$
\underline{\mathbf{I}}=\frac{1}{t}\left[\begin{array}{cc}
{[\eta]} & -\left(\left(1-t^{2}\right)^{-1 / 2}\right)[[\eta]] \\
-\left(\left(1-t^{2}\right)^{-1 / 2}\right)[\eta] & {[\eta]}
\end{array}\right.
$$

where

$$
\begin{gathered}
t=\tanh (a p) \\
a=\frac{l}{\nu}
\end{gathered}
$$


and

$$
\underline{\mathbf{I}}=\left[\begin{array}{c}
\underline{I}_{1} \\
\underline{I}_{2} \\
\underline{I}_{3} \\
\cdot \\
\cdot \\
\cdot \\
\underline{I}_{N}^{\prime} \\
\underline{I}_{1}^{\prime} \\
\underline{I}_{2}^{\prime} \\
\underline{I}_{3}^{\prime} \\
\cdot \\
\cdot \\
\cdot \\
\underline{I}_{N}
\end{array}\right] \quad \text { and } \quad \underline{\mathbf{V}}=\left[\begin{array}{c}
\underline{V}_{1} \\
\underline{V}_{2} \\
\underline{V}_{3} \\
\cdot \\
\cdot \\
\cdot \\
\underline{V}_{N} \\
\underline{V}_{1}^{\prime} \\
\underline{V}_{2}^{\prime} \\
\underline{V}_{3}^{\prime} \\
\cdot \\
\cdot \\
\cdot \\
\underline{V}_{N}
\end{array}\right]
$$

where $[\eta]$ is an $N \times N$ matrix called the characteristic admittance matrix of the line. This matrix is related to the static capacitance matrix of the $N$-wire line in a similar way to the relationship between capacitance per unit length and characteristic admittance of a single line. Thus

$$
[\eta]=\left[\begin{array}{ccccccc}
Y_{11} & -Y_{12} & -Y_{13} & \cdot & \cdot & . & \\
-Y_{12} & Y_{22} & -Y_{23} & \cdot & \cdot & \cdot & \\
-Y_{13} & -Y_{23} & Y_{33} & & & & \\
& & & \cdot & & & \\
& & & & \cdot & & \\
& & & & \cdot & & \\
& & & & & Y_{N-1, N-1} & -Y_{N-1, N} \\
& & & & & -Y_{N-1, N} & Y_{N, N}
\end{array}\right]
$$

This equation gives a complete description of the coupled line structure in terms of its static capacitance matrix.

$$
\underline{\mathbf{I}}=\nu \mathbf{C} \underline{\mathbf{V}}
$$

where $\nu=\frac{\omega}{\beta}$ denotes the phase velocity of the mode. Therefore

$$
\frac{\mathrm{C}}{\epsilon}=7.534 \eta
$$

where $\mathbf{C}$ is the static capacitance matrix of the line given by 


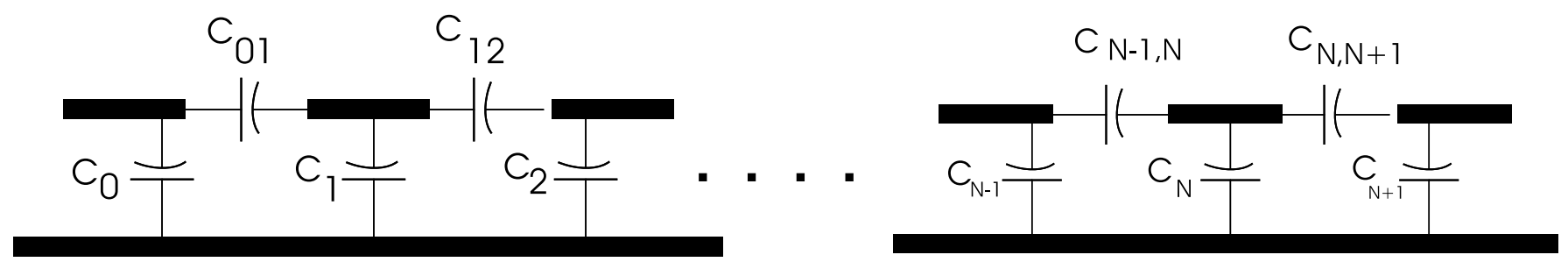

Figure 2.24.: Mutual and self-capacitances of periodic array of interdigitated microstrip conductors.

$$
\mathbf{C}=\left[\begin{array}{cccccccc}
C_{11} & -C_{12} & -C_{13} & \cdot & \cdot & & \\
-C_{12} & C_{22} & -C_{23} & \cdot & \cdot & & & \\
-C_{13} & -C_{23} & C_{33} & & & & \\
& & & \cdot & & & \\
& & & & \cdot & & \\
& & & & \cdot & C_{N-1, N-1} & -C_{N-1, N} \\
& & & & & -C_{N-1, N} & C_{N, N}
\end{array}\right]
$$

where

$$
\begin{aligned}
& C_{11}=C_{1}+C_{12}+C_{23}+\ldots \\
& C_{r r}=C_{r}+C_{1 r}+C_{2 r}+\ldots
\end{aligned}
$$

where the capacitances $C_{1}, C_{2}, C_{3}, \ldots, C_{r}$ are the capacitances per unit length to ground for each of the $N$ lines. $C_{12}, C_{23}$ etc. are the coupling capacitances per unit length between pairs of lines.

\subsubsection{The Combline Filters}

The typical microstrip combline filter of $n^{\text {th }}$ order is shown in Fig. 2.25. The structure consists of quasi transverse electromagnetic TEM-mode transmission lines that are short-circuited at one end and have a lumped capacitance, $C_{L i}$, between the other end of each resonator line element and ground [67]. The principle of operation of the combline filter is as follows. First, if the lumped capacitors were removed then the shunt lines would resonate at their quarter wave frequency. However, the couplings would resonate at this frequency, producing an allstop network. As the capacitors are increased the shunt lines behave as inductive elements and resonate with the capacitors at a frequency below the quarter wave frequency. By applying the boundary condition at the nodes $1^{\prime}, 2^{\prime}, 3^{\prime}, \ldots, N^{\prime}$ are all short circuited then

$$
\underline{V}_{1}^{\prime}=\underline{V}_{2}^{\prime}=\underline{V}_{3}^{\prime}=\underline{V}_{4}^{\prime}=\ldots=\underline{V}_{N}^{\prime}=0
$$




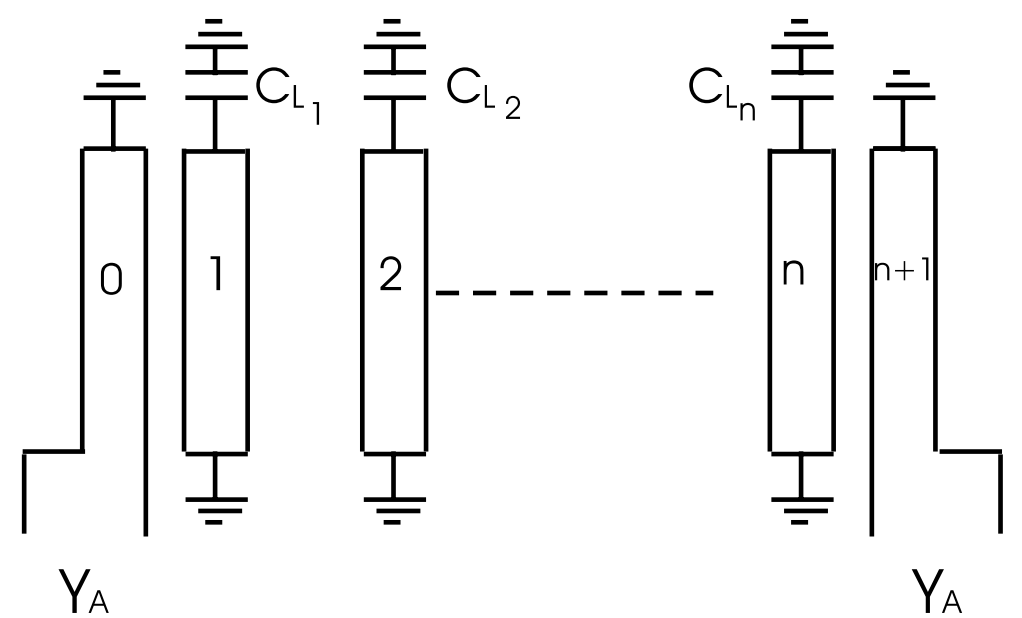

Figure 2.25.: General structure of a microstrip combline bandpass filter.

Adding the lumped capacitances and rearranging the matrix elements, the $N$-port matrix may be written as

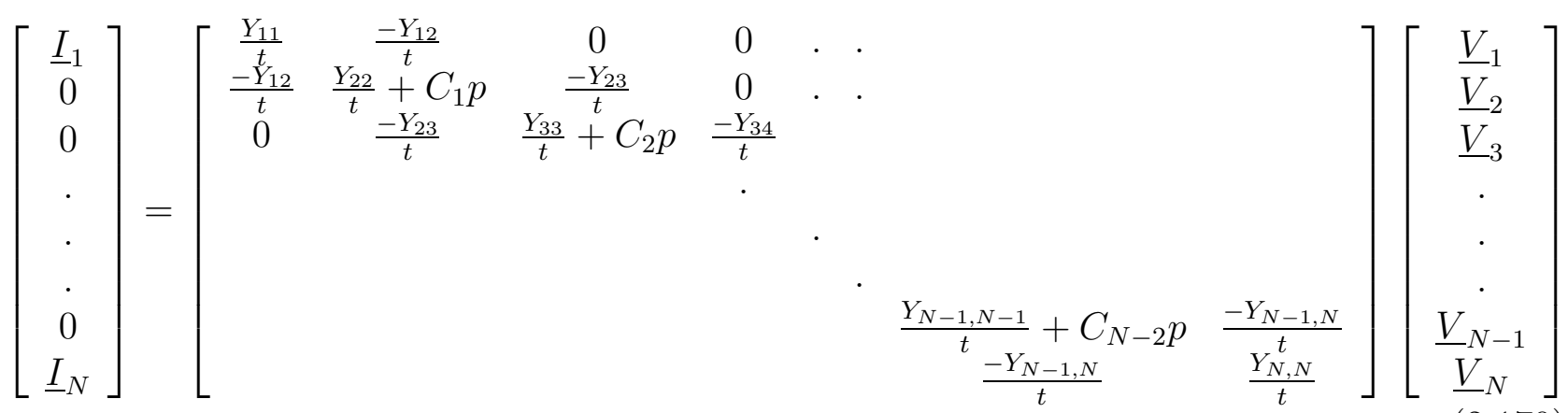

The two-port network impedance could be expressed as follow

$$
\left[\begin{array}{l}
\underline{I}_{1} \\
\underline{I}_{N}
\end{array}\right]=\left[\begin{array}{ll}
Y_{11}^{\prime} & Y_{12}^{\prime} \\
Y_{21}^{\prime} & Y_{22}^{\prime}
\end{array}\right]\left[\begin{array}{l}
V_{1} \\
\underline{V}_{N}
\end{array}\right]
$$

where $Y_{11}^{\prime}, Y_{12}^{\prime}, Y_{21}^{\prime}$ and $Y_{22}^{\prime}$ are founded by solving for $\underline{V}_{2}, \underline{V}_{3}, \underline{V}_{4}, \ldots, \underline{V}_{N-1}$ in terms of $\underline{V}_{1}$, $\underline{V}_{N}, \underline{I}_{1}, \underline{I}_{N}$

\subsubsection{Tunability}

It is desirable in some system applications to shift the pass/rejection band, i.e. to tune the filter. The ability to shift the frequency characteristics of a filter relative to the center frequency of untuned case is called tunability (Fig. 2.26). Tuning of a lumped bandpass filter can be established by changing the value of $L^{\prime}$ s or/and $C^{\prime}$ s. Tuning of microwave distributed filters can be accomplished by varying either the length or the inductive or the capacitive loading of 


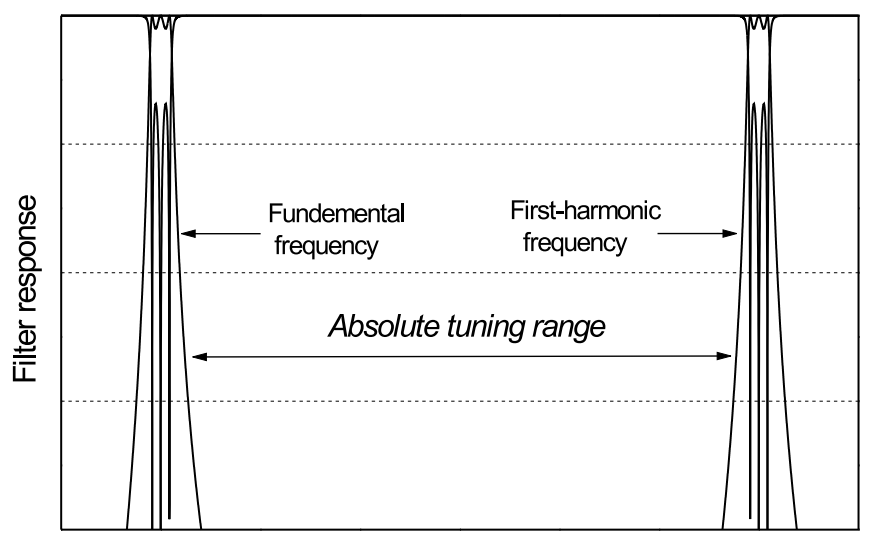

Frequency

Figure 2.26.: Tunability, tunable frequency response.

the resonators, thus changing the the relationships between the impedances and phases inside the filter structure, this defined as system impedance.

The performance of a passive filter is strongly dependent on the system impedance. The system impedance depends on the distribution network configuration and the loads. Therefore, the design of tunable filters involves through system analysis in order to obtain adequate filtering performance of the filter. Not every bandpass filter structure is suited for tuning, and if yes the tunability is topology-dependent. To make a tunable filter care should be taken so that:

- The tuning should be done by a minimum number of tuning elements.

- The filter structure should be simple to be tuned over a broad tuning range.

- Identification of a strategic point within the device structure for optimum tunability.

To have the maximum absolute tuning range (see (Fig. 2.26)), the stopband between first passband (desired) and second passband (unwanted) should be as wide as possible. Thus, it would be possible to shift the filter characteristics at the fundamental frequency as maximum as possible before it overlaps with the frequency characteristics of the first-harmonic passband (Fig. 2.26).

\subsubsection{Center Frequency of a Varactor Loaded Resonator}

A varactor loaded resonator is shown in Fig. 2.27. In order to achieve resonance, the reactance of the transmission line and the varactor must cancel. The input impedance of a transmission line terminated with a load $Z_{L}$ is found to be [68]

$$
Z_{\text {in }}=Z_{0} \frac{Z_{L}+\mathrm{j} Z_{0} \tan (\beta l)}{Z_{0}+\mathrm{j} Z_{L} \tan (\beta l)}
$$

where a sinusoidal excitation is assumed. Here $l$ is the length of the line and $Z_{0}$ is its characteristic impedance. $\beta$ is the propagation constant of the line where 


$$
\beta=\frac{2 \pi}{\lambda}
$$

and

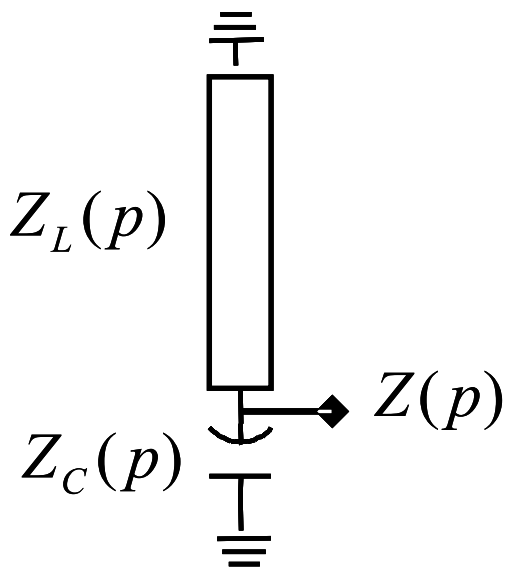

Figure 2.27.: Varactor loaded resonator.

$$
\beta l=\theta
$$

where $\theta$ is the electrical length of the line.

thus, $Z_{\text {in }}$ becomes

$$
Z_{\text {in }}=Z_{0} \frac{Z_{L}+\mathrm{j} Z_{0} \tan (\theta)}{Z_{0}+\mathrm{j} Z_{L} \tan (\theta)}
$$

by short-circuited this line its input impedance becomes

$$
Z_{\text {in }}=\mathrm{j} Z_{0} \tan (\theta)
$$

let $\theta$ to be equal to $a \omega$,

Therefore

$$
Z_{\text {in }}(\mathrm{j} \omega)=\mathrm{j} Z_{0} \tan (a \omega)
$$

Richard's transformation is used to describe the distributed network in terms of complex frequency as follow:

$$
p \rightarrow \alpha \tanh (a p)
$$


or

$$
\omega \rightarrow \alpha \tan (a \omega)
$$

Therefore

$$
Z_{L}(p)=Z_{0} \tanh (a p)
$$

The impedance of a lumped capacitor is

$$
Z_{C}(p)=\frac{1}{C p}
$$

Thus the input impedance of the resonator line $n^{\text {th }}$ for loaded resonator with a lumped capacitor is

$$
Z(p)=Z_{0} \tanh (a p)+\frac{1}{C p}
$$

therefore, the reactance function $X(\omega)$

$$
X(\omega)=Z(j \omega)=Z_{0} \tan (a \omega)-\frac{1}{\omega C}
$$

at resonance, $X(\omega)=0$, thus the necessary capacitance is given by

$$
C=\frac{1}{Z_{0} \omega \tan (a \omega)}
$$

Hence

$$
f=\frac{1}{Z_{0} 2 \pi C \tan (\theta)}
$$

Thus it is possible to adjust the resonance frequency of the resonator by changing the capacitance of the loaded varactor. The tuning of this resonator is limited by its physical length, the internal impedance, the electrical length, and the range of the capacitance of the capacitive load. From the above (2.185) it is quite evident that the smallest capacitance tunes the highest tunable frequency, $f_{\max }$, and the largest capacitance the lowest one, $f_{\min }$. 


\subsection{Tunable Combline Filter}

This section summarize the design procedures for combline filters as they are described in traditional literature in order to make them applicable to the present design goal. The design procedure starts with the specification of the desired filter characteristics over the tuning range. Three main advantages make the combline filter a good candidate to be used as tunable filter:

- Compact size because of the lumped capacitor: with lumped capacitance, resonator length become less than $\lambda / 4$, the larger the capacitance, the shorter length of the resonator. The

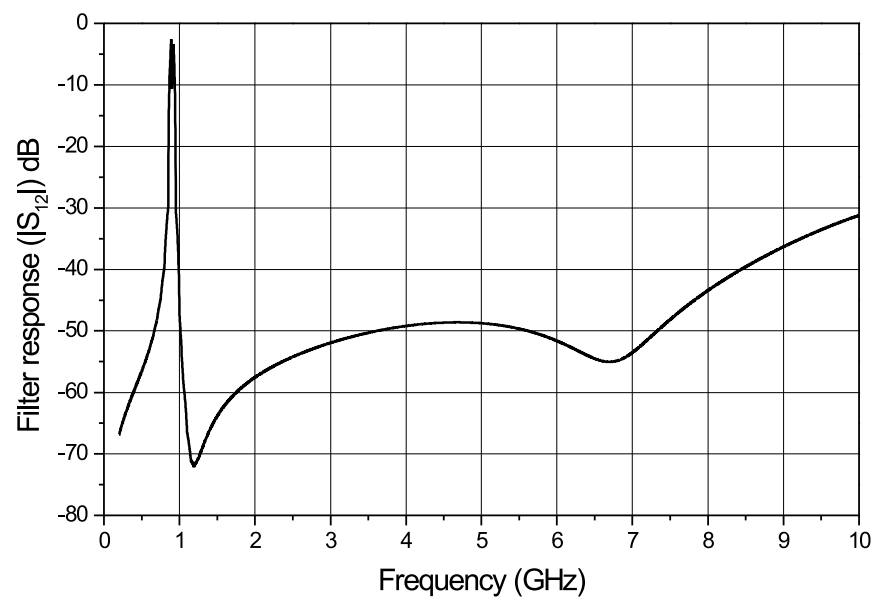

(a) Lower tuning range at $f_{1}$.

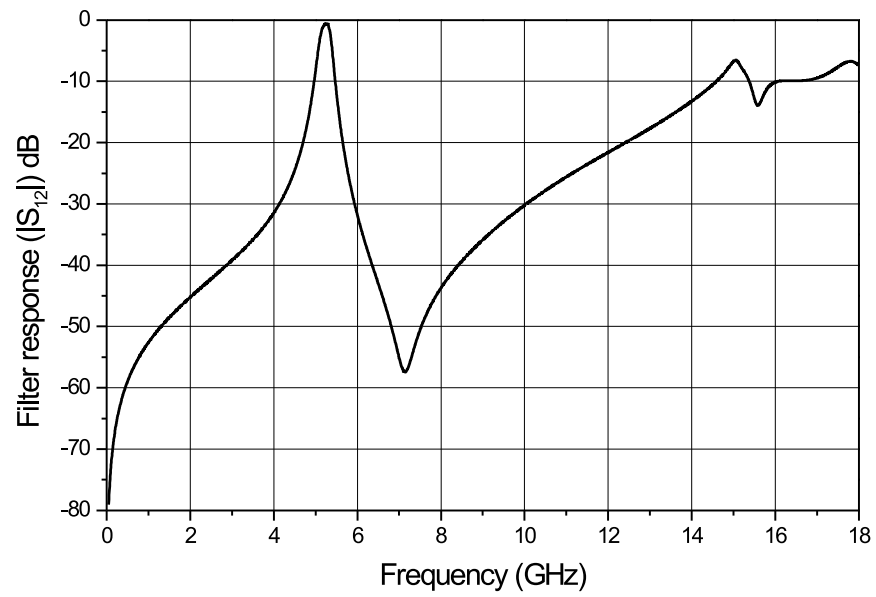

(b) Upper tuning range at $f_{2}$.

Figure 2.28.: Combline filter characteristic 
minimum resonator line length could be limited by the decrease of the unloaded quality factor of the resonator and a requirement for heavy capacitive loading

- A wider stopband between first passband (desired) and second passband (unwanted): if the resonator lines of $\lambda / 8$ long centered at frequency of $f_{0}$, the second-harmonic unwanted passband will be centered over $4 f_{0}$. Thus, more than $300 \%$ tuning range is possible before the upper range spectrum overlaps with the first second passband spectrum (see Fig. 2.28(b) and Fig. 2.28(a)).

- The attenuation will be high above the primary passband and steep rate of cutoff at the upper sideband. The higher the order, the sharper the attenuation.

\subsubsection{Filter Equivalent Circuit}

Hunter and Rhodes [69] propose a three steps transformation of the filter equivalent circuit consisting of

1. The formulation of the admittance inverter equivalent circuit of the filter.

2. Scaling the entire network admittance by $\tan (\theta) / \tan \left(\theta_{0}\right)$.

3. Transforming the previous network to the lowpass prototype with admittance inverters by the inverse of a bandpass transformation.

The coupled line array consists of shunt resonators circuited stubs coupled via series circuited stubs as shown in Fig. 2.29(a). By adding shunt capacitor $C_{r}$ to ground at $r^{\text {th }}$ node the equivalent circuit of the filter is obtained, see Fig. 2.29(b).

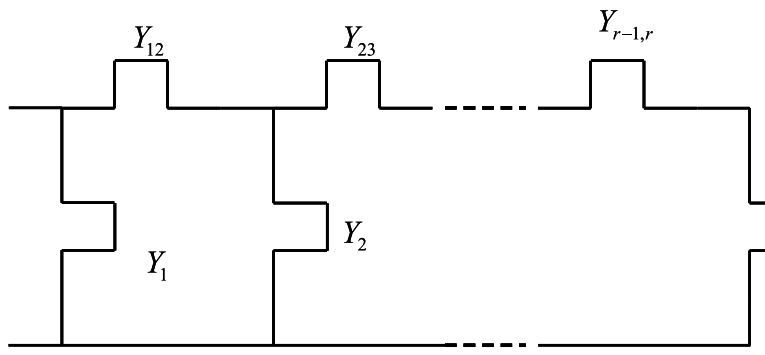

(a) The coupled short circuited lines.

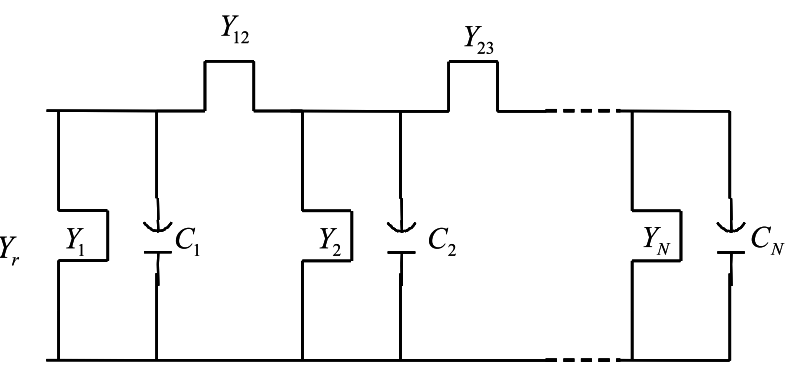

(b) The combline filter.

Figure 2.29.: Equivalent circuit of stubs representations

The lowpass prototype in common is a ladder network. By applying Kuroda's identities, the unit element acted as an impedance inverter by converting a series impedance to a parallel admittance and vice versa. Assume that the admittance inverters have been applied to the ladder network, then all the series impedances can be represented by parallel admittance, therefore the general lowpass prototype is shown in Fig. 2.30, where $C_{L r}$ is elements value of the prototype, which may represents a capacitor or an inductor. 


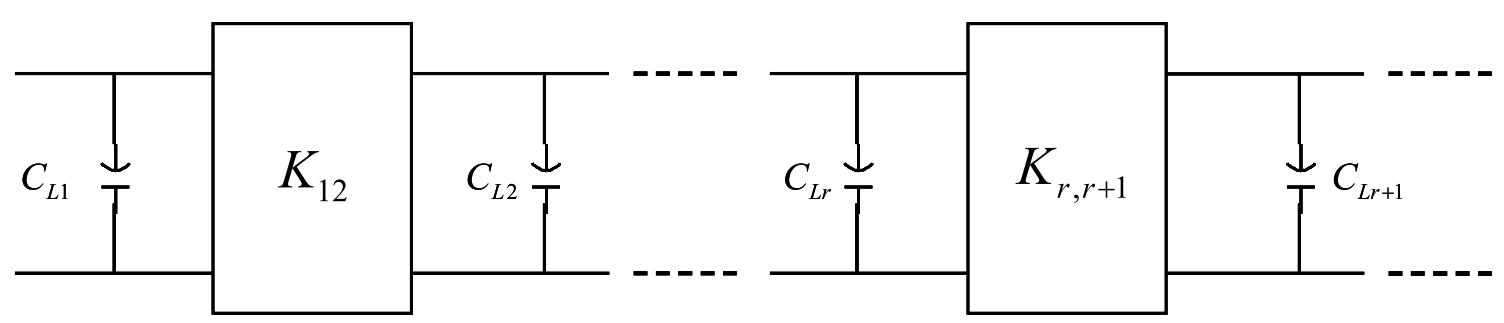

Figure 2.30.: The corresponding lowpass prototype filter of a ladder network.

The filter equivalent circuit will be transformed back to the previous lowpass prototype with admittance inverters and using the inverse of a bandpass transformation. This done as follows: The combline filter equivalent circuit between the two nodes $r^{t h}$ and $(r+1)^{t h}$ is shown in Fig. 2.31(a). The resonators are composed of short circuited stubs in parallel with lumped capacitors. If the series coupling stubs $Y_{r, r+1}$ are now shunted by identical elements but with opposite sign, as shown in Fig. 2.31(b) It can be seen that the transfer matrix of the $\pi$-network of stubs between the dotted lines is given by

$$
\begin{aligned}
\mathbf{T} & =\left[\begin{array}{cc}
1 & 0 \\
\frac{\mathrm{j} Y_{r, r+1}}{\tan (\theta)} & 1
\end{array}\right]\left[\begin{array}{cc}
1 & \mathrm{j} \tan (\theta) \\
0 & 1
\end{array}\right]\left[\begin{array}{cc}
1 & 0 \\
\frac{\mathrm{j} Y_{r, r+1}}{\tan (\theta)} & 1
\end{array}\right] \\
& =\left[\begin{array}{cc}
0 & \frac{\mathrm{j} \tan (\theta)}{Y_{r, r+1}} \\
\frac{\mathrm{j} Y_{r, r+1}}{\tan (\theta)} & 0
\end{array}\right] \\
& =\left[\begin{array}{cc}
0 & \mathrm{j} / K_{r, r+1} \\
\mathrm{j} K_{r, r+1} & 0
\end{array}\right]
\end{aligned}
$$

which is the transfer matrix of an admittance inverter of admittance.

$$
K_{r, r+1}=\frac{Y_{r, r+1}}{\tan (\theta)}
$$

Hence, the $\pi$-network is equivalent to admittance inverter. Thus the $\pi$-network is replaced by an impedance inverter as shown in Fig. 2.31(c). The equivalent circuit of the filter is shown in Fig. 2.32 where $Y_{r r}$ is given by

$$
Y_{r r}=Y_{r}+Y_{r-1, r}+Y_{r, r+1}
$$

Now, the admittance of the $r^{\text {th }}$ resonator is given by

$$
Y_{r}^{\prime}=\mathrm{j} \omega C_{r}-\frac{\mathrm{j} Y_{r r}}{\tan (\theta)}
$$

By comparing the equivalent circuit of the filter with its lowpass prototype (Fig. 2.30 with Fig. 2.32) one can conclude that a shunt capacitor in the prototype has been transformed to shunt capacitor of value $C_{r}$ and shunt short circuited admittance $Y_{r r}$ as follow 


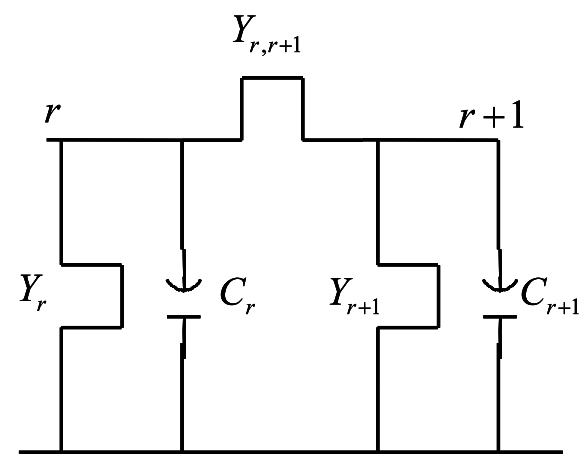

(a)
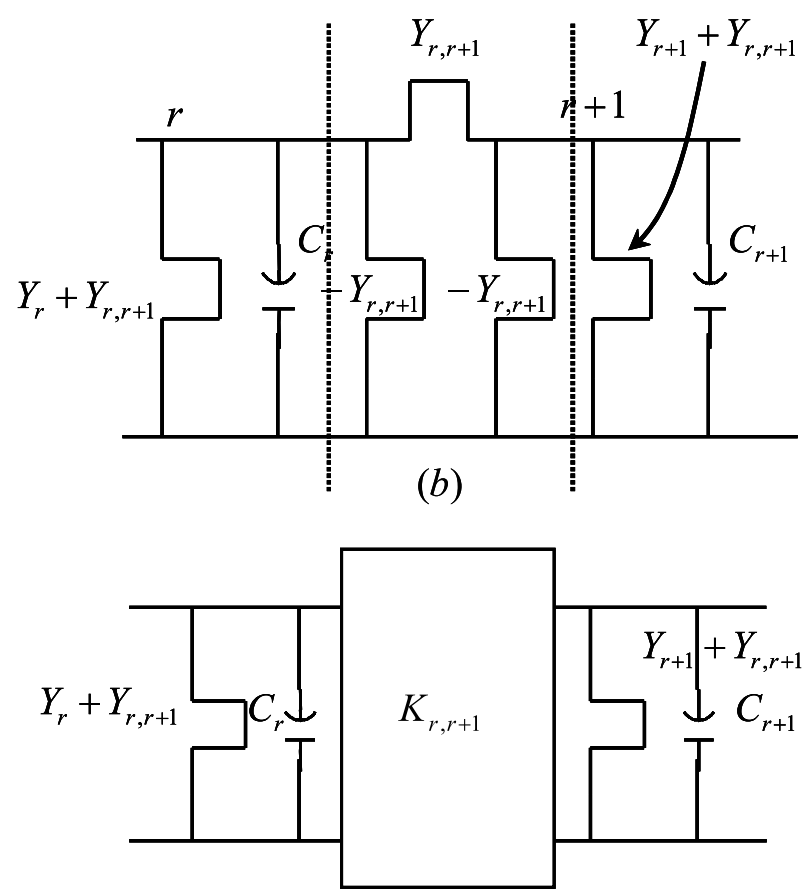

(c)

Figure 2.31.: Formulation of inverters in the combline filter.

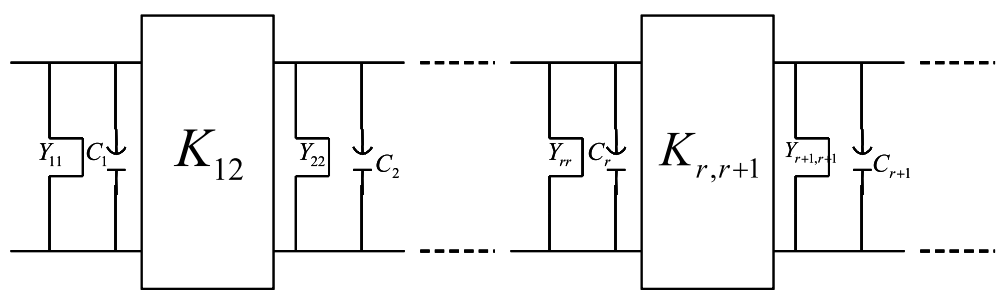

Figure 2.32.: Combline Filter equivalent circuit. 


$$
C_{L r} \quad \rightarrow \quad C_{r}-\frac{Y_{r r}}{\omega \tan (\theta)}
$$

where $C_{L r}$ is the $r^{\text {th }}$ capacitor in the lowpass prototype.

$Y_{r, r+1}$ is frequency dependent, so for an octave tuning this will result in a $3.3: 1$ (that is of around $70 \%$ ) deviation. Thus the filter performance will not be kept along the tuning range.

To remove the frequency dependency from the inverters, the entire network admittance including the source and the load is scaled by a factor $\tan (\theta) / \tan \left(\theta_{0}\right)$ where $\theta_{0}$ is the electrical length of the resonators at the center frequency $\omega_{0}$ of the filter at zero-bias. Thus

$$
K_{r, r+1}=\frac{Y_{r, r+1}}{\tan \left(\theta_{0}\right)}
$$

and

$$
Y_{r}^{\prime}=\frac{\mathrm{j}}{\tan \left(\theta_{0}\right)}\left[\omega C_{r} \tan (\theta)-Y_{r r}\right]
$$

The new transformation from lowpass to bandpass is now given by

$$
\omega C_{L r} \rightarrow\left[\frac{\omega C_{r} \tan (\theta)}{\tan \left(\theta_{0}\right)}-\frac{Y_{r r}}{\tan \left(\theta_{0}\right)}\right]
$$

Thus

$$
\omega \rightarrow\left[\frac{\omega C_{r} \tan (\theta)}{C_{L r} \tan \left(\theta_{0}\right)}-\frac{Y_{r r}}{C_{L r} \tan \left(\theta_{0}\right)}\right]
$$

Let

$$
\begin{gathered}
\alpha=\frac{Y_{r r}}{C_{L r} \tan \left(\theta_{0}\right)} \\
\beta=\frac{C_{r}}{Y_{r r}}
\end{gathered}
$$

Therefore

$$
\omega \rightarrow \alpha[\beta \omega \tan (\theta)-1]
$$


The $\omega=0$ is the lowpass prototype maps to $\omega_{0}$ in the combline filter. Thus

$$
\beta=\frac{1}{\omega_{0} \tan \left(\theta_{0}\right)}
$$

As yet, the frequency dependance of the admittance inverters has not been removed from the filter but merely scaled into the terminating resistors of the source/load which now have a conductance of value

$$
G_{L}=G_{S}=\frac{\tan \left(a \omega_{0}\right)}{\tan (a \omega)}
$$

where $G_{L}, G_{S}$ are the conductance of values of the source and the load respectively.

Thus, to solve this problem the internal nodal admittance of the filter are scaled. This could be done by introducing an non-resonating transformer element at the input and the output of the filter. Now, the input/output coupling network is shown in Fig. 2.33 it will now be assumed, for simplicity, that the network is symmetrical, i.e. $Y_{0}=Y_{1}$.

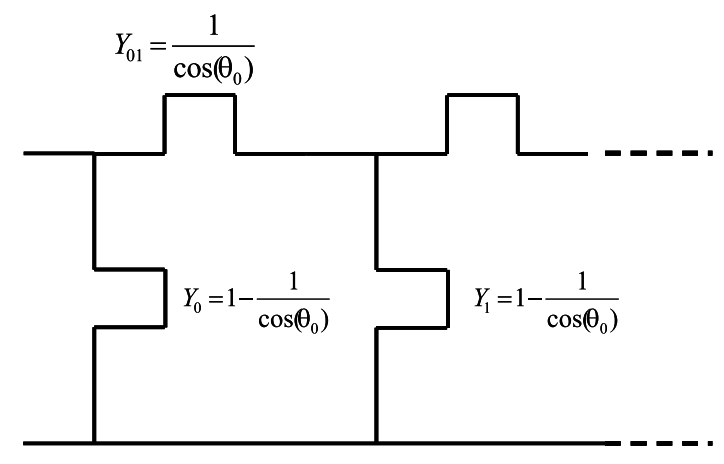

Figure 2.33.: Input-output network.

Evaluating the input admittance of this network, looking back into the load via $Y_{1}$ and after scaling by the factor $\tan (a \omega) / \tan \left(a \omega_{0}\right)$, thus

$$
\mathbf{T}=\left[\begin{array}{cc}
1 & 0 \\
\frac{Y_{1}}{t} & 1
\end{array}\right]\left[\begin{array}{cc}
1 & \frac{t}{Y_{01}} \\
0 & 1
\end{array}\right]\left[\begin{array}{cc}
1 & 1 \\
\frac{Y_{0}}{t} & 1
\end{array}\right]
$$

hence real and imaginary part of the effective admittance are given

$$
\begin{gathered}
\Re\left\{Y_{i n}(\mathrm{j} \omega)\right\}=\frac{\tan (a \omega)}{\tan \left(a \omega_{0}\right)\left[\left[1+Y_{0} / Y_{01}\right]^{2}+\tan ^{2}(a \omega) / Y_{01}^{2}\right]} \\
\Im\left\{Y_{i n}(\mathrm{j} \omega)\right\}=\frac{-\left[\left[Y_{0}\left[1+Y_{0} / Y_{01}\right]\right]\left[2+Y_{0} / Y_{01}\right]+\tan ^{2}(a \omega)\left[\left[1+Y_{0} / Y_{01}\right] / Y_{01}\right]\right]}{\tan \left(a \omega_{0}\right)\left[\left[1+Y_{0} / Y_{01}\right]^{2}+\tan ^{2}(a \omega) / Y_{01}^{2}\right]}
\end{gathered}
$$

For a perfect match at $\omega=\omega_{0}$ it requires that 


$$
\begin{aligned}
& \Re\left\{Y_{\text {in }}(\mathrm{j} \omega)\right\}=1 \\
& \Im\left\{Y_{\text {in }}(\mathrm{j} \omega)\right\}=0
\end{aligned}
$$

From (2.203) and (2.204),

$$
\begin{gathered}
{\left[1+Y_{0} / Y_{01}\right]^{2}+\tan ^{2}\left(a \omega_{0}\right) / Y_{01}^{2}=1} \\
Y_{0}^{2}+2 Y_{0} Y_{01}+\tan ^{2}\left(a \omega_{0}\right)=0
\end{gathered}
$$

Substituting (2.208) into (2.203)

$$
\Re\left\{Y_{i n}(\mathrm{j} \omega)\right\}=\frac{\tan (a \omega)}{\tan \left(a \omega_{0}\right)\left[1-\frac{\tan ^{2}(a \omega)}{Y_{01}^{2}}+\frac{\tan ^{2}\left(a \omega_{0}\right)}{Y_{01}^{2}}\right]}
$$

with $a \omega=\theta$, and after simple manipulation

$$
\Re\left\{Y_{\text {in }}(\mathrm{j} \omega)\right\}=\frac{Y_{01}^{2} \sin (\theta) \cos (\theta) \cos ^{3}\left(\theta_{0}\right)}{\sin \left(\theta_{0}\right)\left[Y_{01}^{2} \cos ^{2}(\theta) \cos ^{2}\left(\theta_{0}\right)+\cos ^{2}\left(\theta_{0}\right)-\cos ^{2}(\theta)\right]}
$$

Now, if the denominator is forced to be frequency invariant, $\Re\left\{Y_{\text {in }}(\mathrm{j} \omega)\right\}$ will vary slowly over as a function of frequency. Thus the best choice by inspection for $Y_{01}$ is

$$
Y_{01}=\frac{1}{\cos \left(\theta_{0}\right)}
$$

and

$$
\Re\left\{Y_{i n}(\mathrm{j} \omega)\right\}=\frac{\sin (2 \theta)}{\sin \left(2 \theta_{0}\right)}
$$

Substituting (2.211) in (2.208) yields

$$
Y_{0}=Y_{1}=1-\frac{1}{\cos \left(\theta_{0}\right)}
$$

and

$$
\Im\left\{Y_{i n}(\mathrm{j} \omega)\right\}=\frac{\cos (2 \theta)-\cos \left(2 \theta_{0}\right)}{\sin \left(2 \theta_{0}\right)}
$$

Under these conditions, the load admittance looking back from the filter in $Y_{\text {in }}$ and after scaling by $\tan (a \omega) / \tan \left(a \omega_{0}\right)$ is given by 


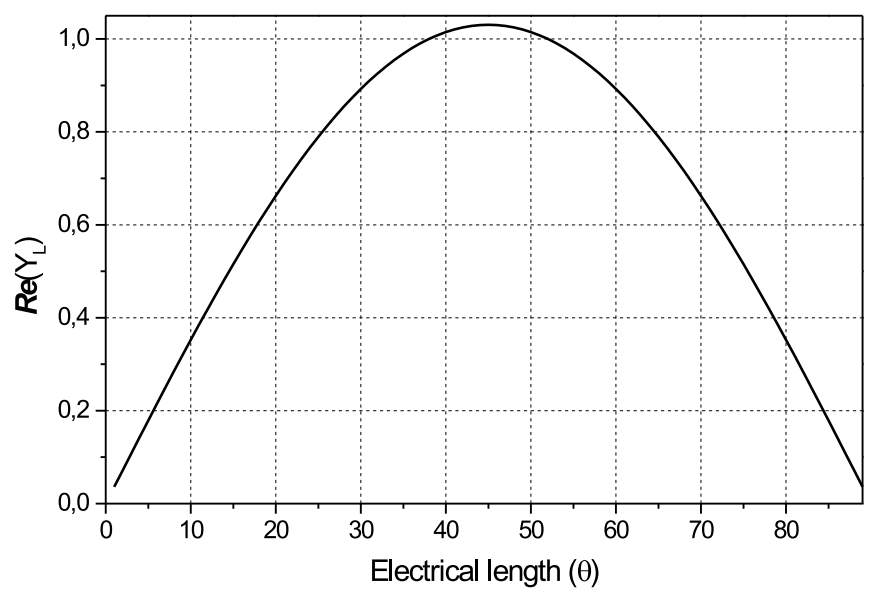

Figure 2.34.: Real part of the input admittance as a function of the electrical length.

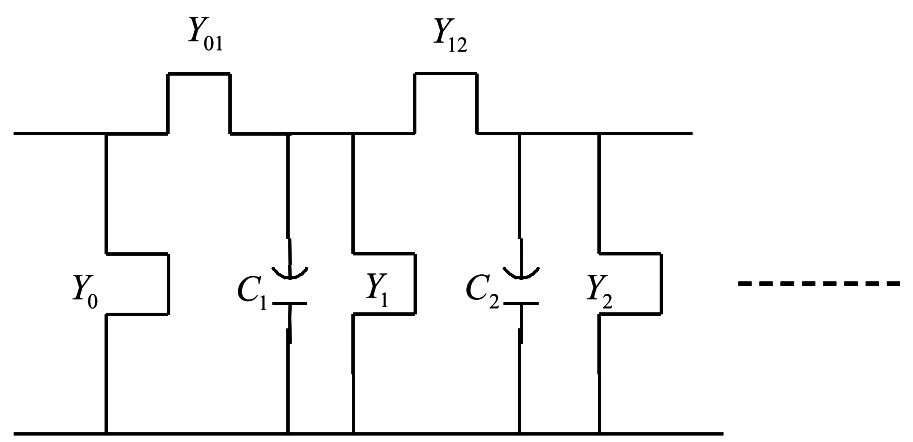

Figure 2.35.: Input port of the filter after scaling. 


$$
Y_{i n}(\theta)=\frac{\sin (2 \theta)}{\sin \left(2 \theta_{0}\right)}+\frac{\mathrm{j} \cos (2 \theta)-\cos \left(2 \theta_{0}\right)}{\sin \left(2 \theta_{0}\right)}
$$

In the next lines the derivation of the passband bandwidth will be performed as follows: The band-edges at \pm 1 in the lowpass prototype map into the band-edges at $\omega_{1}$ and $\omega_{2}$ in the combline filter, i.e.

$$
\begin{aligned}
& -1 \rightarrow \alpha\left[\beta \omega_{1} \tan \left(\theta_{1}\right)-1\right] \\
& +1 \rightarrow \alpha\left[\beta \omega_{2} \tan \left(\theta_{2}\right)-1\right]
\end{aligned}
$$

Let

$$
\begin{aligned}
\theta & =a \omega \\
\omega_{1} & =\omega_{0}-\left\{\frac{\Delta \omega}{2}\right\} \\
\omega_{2} & =\omega_{0}+\left\{\frac{\Delta \omega}{2}\right\}
\end{aligned}
$$

where $\Delta \omega$ is the passband bandwidth.

Inserting equations (2.219), (2.219), (2.220) into equations (2.216), (2.217) yields

$$
\begin{aligned}
& -1=\alpha\left[\beta\left[\omega_{0}-\frac{\Delta \omega}{2}\right] \tan \left(\theta_{0}-\frac{a \Delta \omega}{2}\right)-1\right] \\
& +1=\alpha\left[\beta\left[\omega_{0}+\frac{\Delta \omega}{2}\right] \tan \left(\theta_{0}+\frac{a \Delta \omega}{2}\right)-1\right]
\end{aligned}
$$

For narrow bandwidth $\Delta \omega_{0}<<\omega_{0}$ and by using the approximation for the trigonometric function yields

$$
\tan \left(\theta_{0}+\frac{a \Delta \omega}{2}\right)=\frac{\tan \left(\theta_{0}\right)+\frac{a \Delta \omega}{2}}{1-\left(\frac{a \Delta \omega}{2}\right) \tan \left(\theta_{0}\right)}
$$

From equations 2.221, 2.222 and 2.223 hence,

$$
\begin{aligned}
& -1=\alpha\left[\beta\left[\omega_{0}-\frac{\Delta \omega}{2}\right]\left\{\tan \left(\theta_{0}\right)-\frac{a \Delta \omega}{2}\left[1+\tan ^{2}\left(\theta_{0}\right)\right]\right\}-1\right] \\
& +1=\alpha\left[\beta\left[\omega_{0}+\frac{\Delta \omega}{2}\right]\left\{\tan \left(\theta_{0}\right)+\frac{a \Delta \omega}{2}\left[1+\tan ^{2}\left(\theta_{0}\right)\right]\right\}-1\right]
\end{aligned}
$$

Solving equations $2.224,2.225$ simultaneously, yields

$$
\Delta \omega=\frac{2 \omega_{0} \tan \left(\theta_{0}\right)}{\alpha\left\{\tan \left(\theta_{0}\right)+\theta_{0}\left[1+\tan ^{2}\left(\theta_{0}\right)\right]\right\}}
$$




\subsubsection{Narrowband Filter Design}

For a narrow percentage bandwidth, $\alpha$ will be large

$$
\alpha=\frac{2 \omega_{0} \tan \left(\theta_{0}\right)}{\Delta \omega\left\{\tan \left(\theta_{0}\right)+\theta_{0}\left[1+\tan ^{2}\left(\theta_{0}\right)\right]\right\}}
$$

and since

$$
Y_{r r}=\alpha C_{L r} \tan \left(\theta_{0}\right)
$$

a high values for the shunt admittances would be obtained. To solve this problem the admittance level of the filter is now scaled at each internal node in order to achieve realizable element values for narrow passband bandwidths. After scaling the $r^{\text {th }}$ internal node by a factor $n_{r}^{2}$ a typical coupling network is obtained between the nodes $r$ and $r+1$ shown in Fig. 2.36 with the following transfer matrix

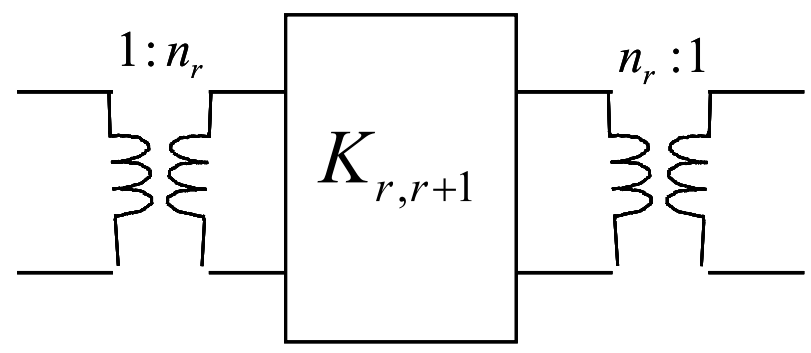

Figure 2.36.: Coupling network between internal nodes of the filter.

$$
\begin{aligned}
\mathbf{T} & =\left[\begin{array}{cc}
1 / n_{r} & 0 \\
0 & n_{r}
\end{array}\right]\left[\begin{array}{cc}
0 & \mathrm{j} / K_{r, r+1} \\
\mathrm{j} K_{r, r+1} & 1
\end{array}\right]\left[\begin{array}{cc}
n_{r} & 0 \\
0 & 1 / n_{r}
\end{array}\right] \\
& =\left[\begin{array}{cc}
0 & \mathrm{j} / n_{r} . n_{r+1} K_{r, r+1} \\
\mathrm{j} n_{r} . n_{r+1} K_{r, r+1} & 0
\end{array}\right]
\end{aligned}
$$

Thus

$$
K_{r, r+1} \quad \rightarrow \quad n_{r} . n_{r+1} K_{r, r+1}
$$

or

$$
K_{r, r+1}=n_{r} . n_{r+1} Y_{r, r+1} / \tan \left(\theta_{0}\right)
$$

The scaling constant becomes 


$$
\alpha=\frac{n_{r}^{2}\left[Y_{r}+Y_{r-1, r}+Y_{r, r+1}\right]}{C_{L r} \tan \left(\theta_{0}\right)}
$$

Now the ideal transformers which remain at the input and the output of the filter must be absorbed into the input and output coupling. To achieve this and to preserve the correct frequency variation of $Y_{L}$ The new values of the $Y_{0}, Y_{1}, Y_{01}$ must be

$$
\begin{aligned}
Y_{0} & =1-\frac{1}{n_{1} \cos \left(\theta_{0}\right)} \\
Y_{01} & =\frac{1}{n_{1} \cos \left(\theta_{0}\right)} \\
Y_{1} & =\frac{1}{n_{1}^{2}}-\frac{1}{n_{1} \cos \left(\theta_{0}\right)}
\end{aligned}
$$

Now the admittance matrix of the combline filter in (2.237) becomes

$$
\mathbf{Y}=\left[\begin{array}{ccccccc}
\frac{1}{t} & \frac{-1}{t \cos \left(\theta_{0}\right)} & 0 & 0 & \cdot & & \\
\frac{-1}{t \cos \left(\theta_{0}\right)} & \frac{1}{t}+\frac{Y_{11}}{t}+p C_{1} & \frac{-Y_{12}}{t} & 0 & \cdot & \cdot & \\
0 & \frac{-Y_{12}}{t} & \frac{Y_{22}}{t}+p C_{2} & \frac{-Y_{23}}{t} & & & \\
& & & \cdot & & & \\
& & & & \cdot & \cdot & \\
& & & & & \frac{Y_{N, N}}{t}+p C_{N} & \frac{-Y_{N, N+1}}{t} \\
& & & & & \frac{-Y_{N, N+1}}{t} & \frac{Y_{N+1, N+1}}{t}
\end{array}\right]
$$

\subsubsection{Filter Parameters Selection}

The filter tunable parameters will be now selected to ensure a broadband tunability keeping the filter performance in a good shape.

\section{Center Frequency}

The variation of coupling and other filter parameters over the entire tuning range well be optimized when the filter is designed at the midband of the tuning range. Moreover, since the tuning element exhibits a low quality factor at low frequency tuning range the rejection requirements are harder to attain, thus the filter should be designed as close as possible to the lower tuning range. The frequency $f_{0}$ is selected to be

$$
f_{0}=\sqrt{f_{1} f_{2}}
$$

where $f_{1}$ and $f_{2}$ are the tuning bandwidth lower and upper limits. A smaller value of $f_{0}$ may be chosen on the assumption that requirements at the highest part of the tuning range will be met without problems. 


\section{Passband Bandwidth}

The next important parameter in tuning a bandpass filter is its passband bandwidth. Some applications required to have a tunable passband, the other requires to keep it constant over the tuning. Since it depends on the coupling between the lines, the passband bandwidth is expected to be change in the same manner. The frequency dependency of the passband bandwidth is shown in Fig. 2.37. It can be seen that the bandwidth posses a turning point for a value of $\theta$

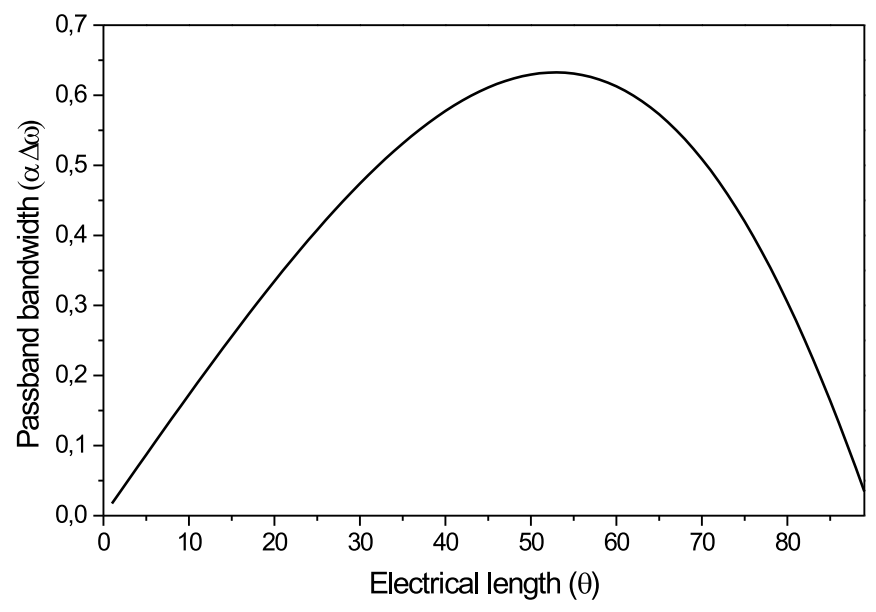

Figure 2.37.: The passband bandwidth as a function of the operating frequency.

between 0 and $90^{\circ}$. The turning point occurs roughly at $\theta=52^{\circ}$. This value of $\theta$ corresponds to the frequency at which bandwidth is maximized. Thus, to have less than $20 \%$ bandwidth deviation in a tunable combline filter, its resonator should be designed to be $52^{\circ}$. TorregrosaPenalva et al. have proposed in [70] the possible range of the resonators electrical length to be designed at. They have defined the normalized instantaneous bandwidth $\Delta \omega_{n}$ as follows:

$$
\begin{aligned}
\Gamma & =10 \log _{10}\left[\Delta \omega_{n}\right] \\
& =10 \log _{10}\left[\frac{\theta \tan (\theta)}{\left\{\tan (\theta)+\theta\left[1+\tan ^{2}(\theta)\right]\right\}}\right]
\end{aligned}
$$

Equation (2.240) is depicted in Fig. 2.38. Due to the linear relationship between electrical length and frequency, this equation also shows the instantaneous bandwidth dependence on the tuning frequency.

To find the electrical length which is required for a specific return loss, one has to determine $\theta_{\min _{1}}$ and $\theta_{\text {min }_{2}}$ as they define graphically in Fig. 2.38. Now let

$$
\Delta \Gamma=10 \log _{10}\left[\frac{\Delta \omega_{\max }}{\Delta \omega_{\min }}\right]
$$

and 


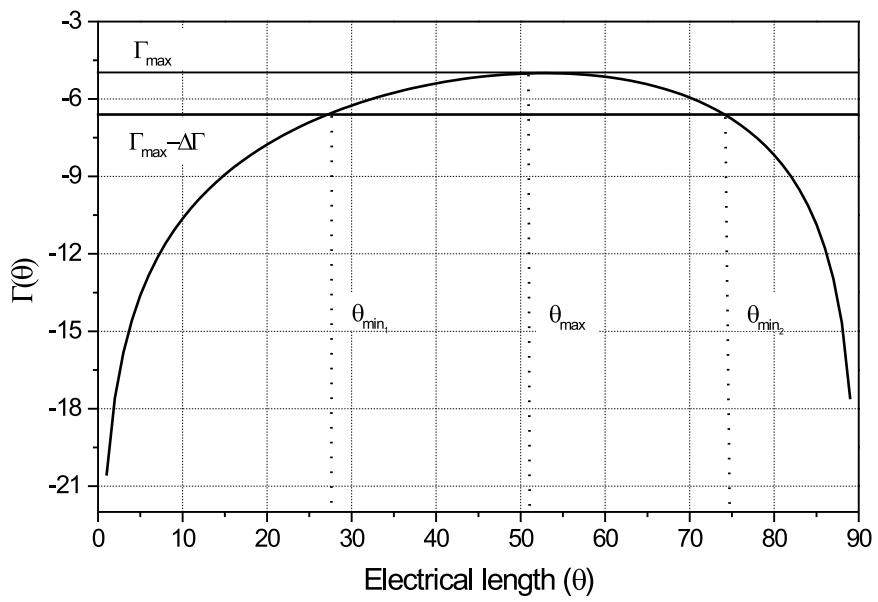

Figure 2.38.: Normalized instantaneous bandwidth.

$$
\left\{\theta_{\min _{1}}, \theta_{\min _{2}}\right\}=\Gamma^{-1}\left[\Gamma\left(\theta_{\max }\right)-\Delta \Gamma\right]
$$

Then

$$
\theta_{\min _{1}} \leq \theta_{1} \leq \theta_{0} \leq \theta_{2} \leq \theta_{\min _{2}}
$$

This constraint can be written as follows

$$
\frac{\theta_{\min _{1}}}{f_{1}} \leq \frac{\theta_{0}}{f_{0}} \leq \frac{\theta_{\min _{2}}}{f_{2}}
$$

or

$$
\theta_{\min _{1}} \frac{f_{0}}{f_{1}} \leq \theta_{0} \leq \theta_{\min _{2}} \frac{f_{0}}{f_{2}}
$$

(2.245) gives the possible ranges of $\theta_{0}$ for a certain return loss. In the same way, the bandwidth can be written as

$$
\Delta \omega\left(\theta_{\min }\right) \frac{\Delta \omega_{n}\left(\theta_{0}\right)}{\Delta \omega_{n}\left(\theta_{\min }\right)} \leq \Delta \omega\left(\theta_{0}\right) \leq \Delta \omega\left(\theta_{\max }\right) \frac{\Delta \omega_{n}\left(\theta_{0}\right)}{\Delta \omega_{n}\left(\theta_{\max }\right)}
$$

(2.246) gives the possible ranges of $\Delta \omega\left(\theta_{0}\right)$ for a certain return loss. 


\section{Capacitance Range}

It is also important to know how much difference between the capacitances of the tuning elements could be. Given

$$
C=\frac{1}{Z_{0} \omega \tan (a \omega)}
$$

According to (2.247) the smallest capacitance $C_{\min }$ tunes the highest tunable frequency $f_{2}$, and the largest capacitance $C_{\max }$ tunes the lowest one, $f_{1}$. Moreover,

$$
C(f) Z_{0} \omega \tan (a \omega)=1
$$

Now, The possible values of the $C_{0}$ where the center frequency of the filter is $f_{0}$ is bounded by

$$
C_{m i d_{2}} \leq C_{0} \leq C_{\text {mid }_{1}}
$$

It is evident that

$$
\begin{gathered}
f_{1}<f_{0}<f_{2} \\
\frac{\theta_{0} f_{1}}{f_{0}}<\theta_{0}<\frac{\theta_{0} f_{2}}{f_{0}}
\end{gathered}
$$

Hence

$$
\tan \left(\frac{\theta_{0} f_{1}}{f_{0}}\right)<\tan \left(\theta_{0}\right)<\tan \left(\frac{\theta_{0} f_{2}}{f_{0}}\right)
$$

According to $(2.250),(2.252)$ can be written as

$$
f_{1} \tan \left(\frac{\theta_{0} f_{1}}{f_{0}}\right)<f_{0} \tan \left(\theta_{0}\right)<f_{2} \tan \left(\frac{\theta_{0} f_{2}}{f_{0}}\right)
$$

dividing $(2.253)$ by $f_{0} \tan \left(\theta_{0}\right)$ yields

$$
\frac{f_{1} \tan \left(\frac{\theta_{0} f_{1}}{f_{0}}\right)}{f_{0} \tan \left(\theta_{0}\right)}<1<\frac{f_{2} \tan \left(\frac{\theta_{0} f_{2}}{f_{0}}\right)}{f_{0} \tan \left(\theta_{0}\right)}
$$


Now since

$$
C_{\min } \leq C_{0} \leq C_{\max }
$$

(2.254) can be written as follows

$$
C_{\max } \frac{f_{1} \tan \left(\frac{\theta_{0} f_{1}}{f_{0}}\right)}{f_{0} \tan \left(\theta_{0}\right)}>C_{0}>\frac{f_{2} \tan \left(\frac{\theta_{0} f_{2}}{f_{0}}\right)}{f_{0} \tan \left(\theta_{0}\right)} C_{m i n}
$$

Thus, (2.256) gives the possible ranges of $C_{0}$ for a certain tuning range.

\section{Insertion and Return Losses}

$$
\begin{gathered}
Y_{i n}(\theta)=\frac{\sin (2 \theta)}{\sin \left(2 \theta_{0}\right)}+\mathrm{j} \frac{\cos (2 \theta)-\cos \left(2 \theta_{0}\right)}{\sin \left(2 \theta_{0}\right)} \\
Y_{i n}(\mathrm{j} \omega)=\Re\left\{Y_{i n}(\mathrm{j} \omega)\right\}+\mathrm{j} \Im\left\{Y_{i n}(\mathrm{j} \omega)\right\}
\end{gathered}
$$

Now, the reflection coefficient can be computed as follows

$$
S_{11}(\mathrm{j} \omega)=\frac{1-Y_{i n}(\mathrm{j} \omega)}{1+Y_{i n}(\mathrm{j} \omega)}
$$

and from the unitary relation, the transmission coefficient is given by

$$
\left|S_{12}(\mathrm{j} \omega)\right|^{2}=1-\left|S_{11}(\mathrm{j} \omega)\right|^{2}
$$

To compute for the turn point in the return and the insertion losses the first derivatives of both the real and the imaginary part of the admittance function with respect to $\theta=a \omega$ are performed and then set to zero, and solve for optimality.

Form (2.205)

$$
\frac{\mathrm{d} \Re\left\{Y_{i n}\right\}(\theta)}{\mathrm{d} \theta}=0
$$

This yields

$$
\frac{\sin (2 \theta) \cdot 0-\sin \left(2 \theta_{0}\right) \cdot 2 \cos (2 \theta)}{\sin ^{2}\left(2 \theta_{0}\right)}=0
$$


Hence

$$
\sin \left(2 \theta_{0}\right) .2 \cos (2 \theta)=0
$$

Thus one can conclude that

$$
\cos (2 \theta)=0
$$

Therefor and since $\theta$ is bounded between $[0, \pi / 2]$ yields

$$
\theta=\frac{\pi}{4}
$$

At $\theta=\frac{\pi}{4}$ The load will be perfectly matched and will consists of a unity real part. Mathematically;

$$
\begin{aligned}
Y_{\text {in }}\left(\theta_{0}\right) & =\Re\left\{Y_{\text {in }}\left(\theta_{0}\right)\right\}+\mathrm{j} \Im\left\{Y_{\text {in }}\left(\theta_{0}\right)\right\} \\
& =1+\mathrm{j} .0 \\
& =1
\end{aligned}
$$

The reflection and the transmission coefficients are

$$
\begin{aligned}
& S_{11}\left(\theta_{0}\right)=1 \\
& S_{12}\left(\theta_{0}\right)=0
\end{aligned}
$$

\section{Passband Ripple}

Consider a lowpass prototype (LPP) factorized transfer function $H_{L P P}(s)$ with $n$ poles $s_{p_{1}}, s_{p_{2}}$, $s_{p_{3}}, \ldots s_{p_{n}}$ and $m$ zeros $s_{z_{1}}, s_{z_{2}}, s_{z_{3}}, \ldots s_{z_{m}}$, given by

$$
H_{L L P}(s)=K \frac{\left(1-s / s_{z_{1}}\right)\left(1-s / s_{z_{2}}\right)\left(1-s / s_{z_{3}}\right) \ldots\left(1-s / s_{z_{n}}\right)}{\left(1-s / s_{p_{1}}\right)\left(1-s / s_{p_{2}}\right)\left(1-s / s_{p_{3}}\right) \ldots\left(1-s / s_{p_{n}}\right)}
$$

This function is scaled to any filter types using the Laplace transformation in equations (2.51) to (2.54. Hence, the poles and zeros will be subject to the same transformation and will be tuned simultaneously in the same manner. It can be seen from substituting (2.54)into (2.271) that the bandpass transfer function has twice as many poles and zeros as the LPP transfer function. In addition it has $(n-m)$ zeros at the origin. For a narrowband, the zeros and the poles patterns have been reproduced at half scale of the bandwidth at $\pm j \omega_{0}$ in case of a narrowband bandpass filter. 


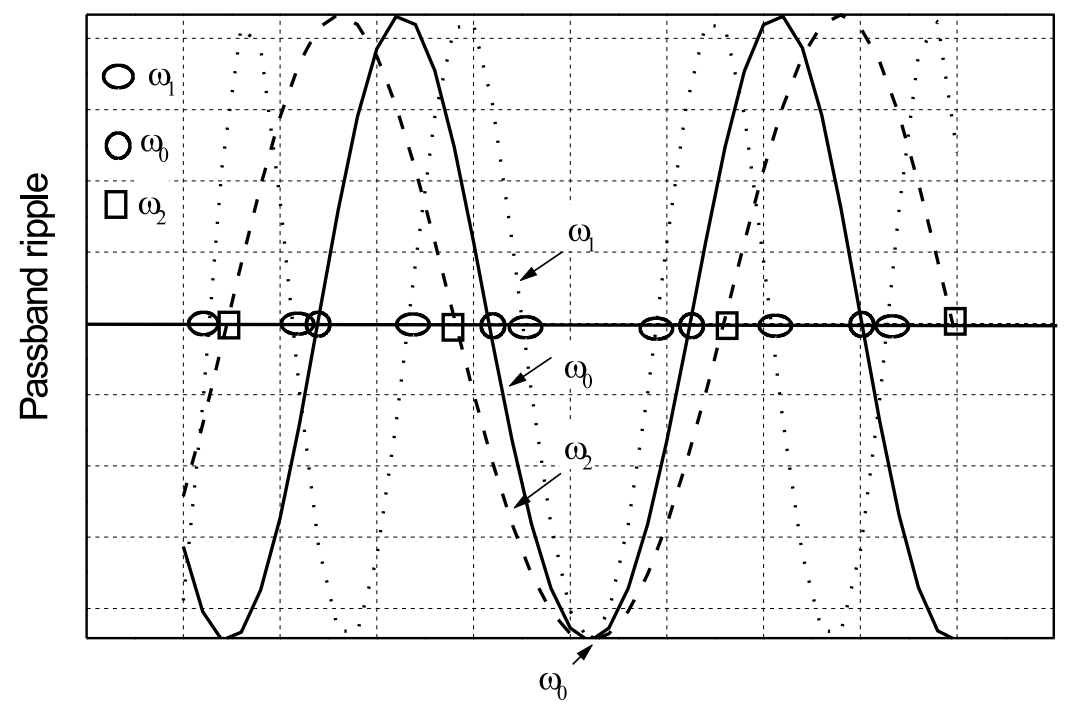

Figure 2.39.: Chebyshev ripple over tuning.

Fig. 2.39 shows the zero's behaviour over the tuning. The filter passband has been kept constant along the tuning, it has been assumed to be narrowband 0.1 . The $\omega_{0}$ corresponds to zero-bias. It has been chosen to be $0.75 \mathrm{rads}^{-1}$. $\omega_{1}, \omega_{2}$ denotes the lower and the upper tuning limits. They have been chosen to be $0.5 \mathrm{rads}^{-1}, 1 \mathrm{rads}^{-1}$, respectively. Assume that the filter has an order $N$ which is equal to the number of the ripples in the passband. When the filter is tuned to the maximum limit the distance between the zeros becomes longer than that one for the unbiased. The number of the ripple thus decreases in the shown window. The opposite happened at the lower limit, where the zeros starched together, causing their numbers to increase, which yields more ripples. 


\subsection{Example}

To illustrate the described design method, assume a first and second order Butterworth lowpass filter prototype.

\subsubsection{First-Order Filter}

The bandpass filter is designed to have a bandwidth $\Delta \omega_{2}$ of 0.78 at the maximum tunable frequency $f_{2}=2.6 \mathrm{GHz}$. The lower tuning part is set at the frequency $f_{1}=1.1 \mathrm{GHz}$ with $\Delta \omega_{1}$ of 0.165. First of all, the midband tuning frequency is chosen according to be the geometric means of the two limits, therefore

$$
f_{0}=\sqrt{f_{1} f_{2}}=1.6912 \quad G H z
$$

Now, the electrical length will be computed as follows: assume that the return loss of the passband wished to vary by $10 \mathrm{~dB}$ across the entire tuning range, thus

$$
\Gamma_{\max }-\Delta \Gamma=10 \mathrm{~dB}
$$

From Fig. 2.38, The values of

$$
\begin{aligned}
& \theta_{\min _{1}}=12^{\circ} \\
& \theta_{\text {min } 2_{2}}=85^{\circ}
\end{aligned}
$$

are calculated and substituted in (2.245) to find the possible ranges of $\Delta \omega\left(\theta_{0}\right)$, the lower and the upper limits are found to be

$$
\begin{aligned}
\theta_{L} & =12^{\circ} \frac{f_{0}}{f_{1}}=0.3220 \mathrm{rad} \\
\theta_{U} & =85^{\circ} \frac{f_{0}}{f_{2}}=0.9650 \mathrm{rad}
\end{aligned}
$$

Again the best choice for $\theta_{0}$ is the geometric means of the extremes design. Hence

$$
\theta_{0}=\sqrt{\theta_{L} \theta_{U}}=0.5574 \mathrm{rad}
$$

Now, the calculation is proceed to find the possible range for the capacitance, from $(2.256)$ the lower and the upper limits of the possible range of $C_{0}$ are

$$
C_{L}=f_{2} \frac{\tan \left(\theta_{0} \frac{f_{2}}{f_{0}}\right)}{f_{0} \tan \left(\theta_{0}\right)} C_{2}=3.8439 p F
$$




$$
C_{U}=f_{1} \frac{\tan \left(\theta_{0} \frac{f_{1}}{f_{0}}\right)}{f_{0} \tan \left(\theta_{0}\right)} C_{1}=2.7708 p F
$$

Like $\theta_{0}$, the geometric means is taken, therefore

$$
C_{0}=\sqrt{C_{U} C_{L}}=3.2635 p F
$$

To compute the internal filter impedance $Z$

$$
Z=\frac{1}{C_{0} 2 \pi f_{0} \tan \left(\theta_{0}\right)}=47 \Omega
$$

Now, it is possible to compute the electrical length which corresponds to the minimum and the maximum tuning ranges. Thus

$$
\begin{aligned}
& \theta_{1}=\tan ^{-1}\left(\frac{1}{C_{1} 2 \pi f_{1} Z}\right)=0.4202 \mathrm{rad} \\
& \theta_{2}=\tan ^{-1}\left(\frac{1}{C_{2} 2 \pi f_{2} Z}\right)=0.7754 \mathrm{rad}
\end{aligned}
$$

The computation of $\Delta \omega_{n_{0}}$ carried out by using (2.239), which is found to be

$$
\Delta \omega_{n_{0}}=0.2487
$$

Also,

$$
\begin{aligned}
& \Delta \omega_{n_{1}}=0.1974 \\
& \Delta \omega_{n_{2}}=0.3039
\end{aligned}
$$

To compute for $\Delta \omega_{0}$, from (2.246), the lower and the upper limits are

$$
\begin{aligned}
\Delta \omega_{0_{L}} & =\Delta \omega_{1} \frac{\Delta \omega_{n_{0}}}{\Delta \omega_{n_{1}}}=0.2078 \mathrm{rad} \\
\Delta \omega_{0_{U}} & =\Delta \omega_{2} \frac{\Delta \omega_{n_{0}}}{\Delta \omega_{n_{2}}}=0.6381 \mathrm{rad}
\end{aligned}
$$


Finally, by taken the geometric means yields

$$
\Delta \omega_{0}=\sqrt{\Delta \omega_{0_{L}} \Delta \omega_{0_{U}}}=0.3641 \mathrm{rad}
$$

As yet, the parameters selections have been. Next, the filter will be design for the following set of specifications:

$$
\begin{aligned}
f_{0} & =1.6912 \mathrm{GHz} \\
\theta_{0} & =0.5574 \mathrm{rad} \\
\Delta \omega_{0} & =0.3641 \mathrm{GHz} \\
C_{0} & =3.2635 \mathrm{pF}
\end{aligned}
$$

The filter of order $n=1$, from Table 2.1, the prototype elements values are

$$
\begin{aligned}
& g_{1}=2 \\
& g_{2}=1
\end{aligned}
$$

computing for $\alpha$ using (2.227)

$$
\alpha=6.5085
$$

Now, $C_{0}$ is normalized to $1 \Omega$, as follows

$$
C_{n}=50 \times C_{0}=1.6318 \times 10^{-10} \quad F
$$

Now, from (2.191) and (2.228)yield the following

$$
Y_{r r}=Y_{1}+Y_{01}+Y_{12}=C_{0} \omega_{0} \tan \left(\theta_{0}\right)=1.0808 \text { mhos }
$$

Hence from (2.233)

$$
n_{r}=2.7400
$$

Now, the elements of the input output coupling circuit will be performed and represented as stubs, from (2.234) to (2.236),

$$
\begin{aligned}
Y_{0} & =0.5699 \text { mhos } \\
Y_{01} & =0.4301 \text { mhos } \\
Y_{1} & =-0.2969 \text { mhos }
\end{aligned}
$$




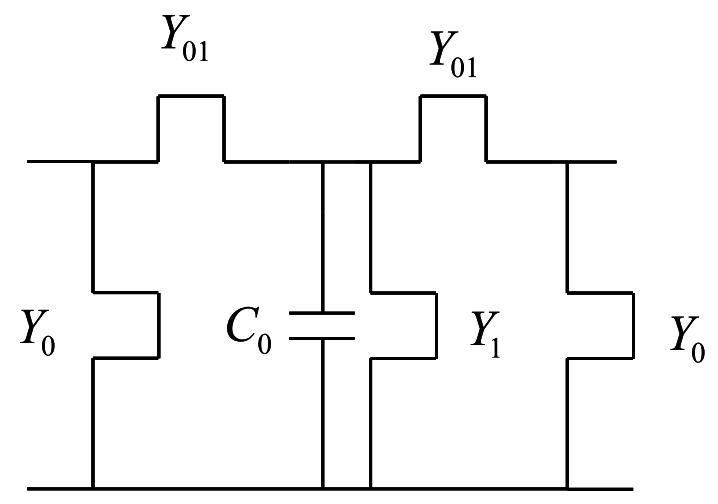

Figure 2.40.: Example: filter equivalent circuit.

The whole filter circuit is shown in Fig. 2.40. $Y_{1}=1.0808$ mhos, this must increased to remove the negative $Y_{1}$ which resulted from the equivalent circuit, thus, $Y_{1}=0.6507-0.1817=0.7839$ mhos.

The transfer matrix of the filter is computed as follows:

$T=\left[\begin{array}{cc}1 & 0 \\ \frac{Y_{0}}{\mathrm{t} \tan (\theta)} & 1\end{array}\right]\left[\begin{array}{cc}1 & \frac{\mathrm{j} \tan (\theta)}{Y_{01}} \\ 0 & 1\end{array}\right]\left[\begin{array}{cc}1 & 0 \\ C_{0} \mathrm{j} \omega & 1\end{array}\right]\left[\begin{array}{cc}1 & 0 \\ \frac{Y_{1}}{\mathrm{j} \tan (\theta)} & 1\end{array}\right]\left[\begin{array}{cc}1 & \frac{\mathrm{j} \tan (\theta)}{Y_{01}} \\ 0 & 1\end{array}\right]\left[\begin{array}{cc}1 & 0 \\ \frac{Y_{0}}{\mathrm{j} \tan (\theta)} & 1\end{array}\right]=\left[\begin{array}{cc}A & B \\ C & D\end{array}\right]$

since it is matched, therefore

$$
Z_{i n}(\theta)=\frac{A+B}{C+D}
$$

Hence, the input reflection coefficient is

$$
S_{11}(\theta)=\frac{Z_{\text {in }}(\theta)-1}{Z_{\text {in }}(\theta)+1}
$$

in decibel

$$
L_{R}=-20 \log _{10}\left(\left|S_{11}(\theta)\right|\right)
$$

and from the unitary condition (A.19)

$$
\begin{aligned}
& \left|S_{12}(\theta)\right|^{2}=1-\left|S_{11}(\theta)\right|^{2} \\
& L_{A}=-20 \log _{10}\left(\left|S_{12}(\theta)\right|\right)
\end{aligned}
$$

The computed performance is shown in Fig. 2.41. The matching varied with less than $10 d B$ across the whole tuning range. The bandwidth of the passband increased constantly as the 


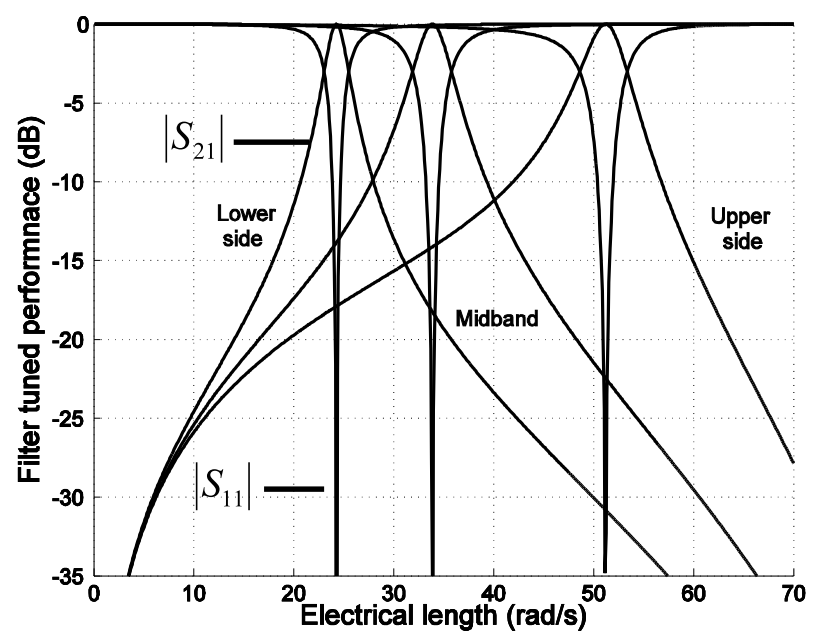

Figure 2.41.: First order computed tuned filter performance, $\Delta \omega_{1}=\theta_{1} / a=168 \mathrm{MHz}$, thus, $a=1.17$, hence, $\Delta \omega_{0}=382 \mathrm{MHz}$ and $\Delta \omega_{2}=719 \mathrm{MHz}$.

frequency goes up. The selectivity of the filter getting poor as the filter tuned for the upper limit, because of the DC pole at the zero frequency, which is present on the lower side.

To compute the static self and mutual capacitances between the parallel coupled lines, the admittance are converted to impedance and matched to $50 \Omega$ by using the following equations:

$$
Z=\frac{1}{Y} \times 50
$$

therefor

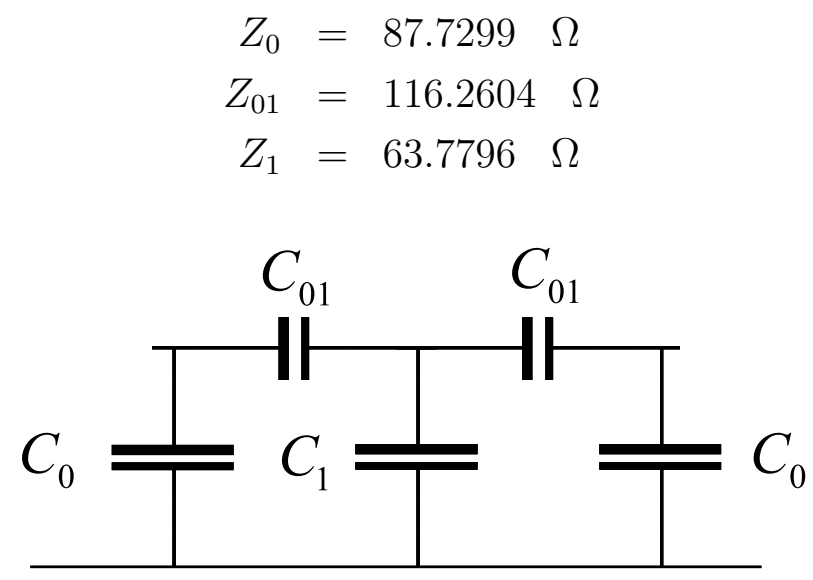

Figure 2.42.: self and mutual static capacitance definitions for the first order filter.

and the capacitance are calculated by

$$
\frac{\sqrt{\epsilon_{r}} C}{\epsilon}=\frac{377}{Z}
$$




$$
\begin{aligned}
& \frac{\sqrt{\epsilon_{r}} C_{0}}{\epsilon}=4.2973 \\
& \frac{\sqrt{\epsilon_{r}} C_{01}}{\epsilon}=3.2427 \\
& \frac{\sqrt{\epsilon_{r}} C_{1}}{\epsilon}=5.9110
\end{aligned}
$$

The definitions of these capacitances are shown in Fig. 2.42.

\subsubsection{Second-Order Filter}

In this sub-section a second order version of the previous specification will be designed. The parameters that have been selected in the first order are not subject to any change because they are order independent. Hence,

$$
\begin{aligned}
f_{0} & =1.6912 \mathrm{GHz} \\
\theta_{0} & =0.5574 \mathrm{rad} \\
\Delta \omega_{0} & =0.3641 \mathrm{GHz} \\
C_{0} & =3.2635 \mathrm{pF}
\end{aligned}
$$

The filter equivalent circuit is shown in Fig. 2.43

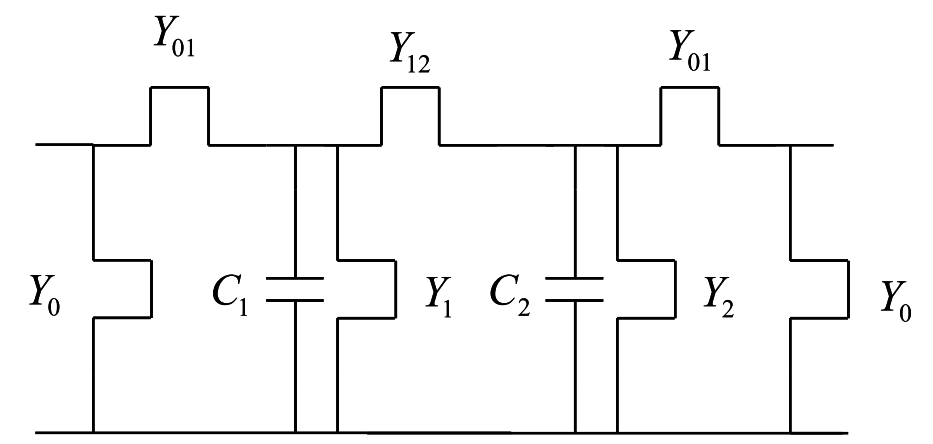

Figure 2.43.: Example: second-order filter equivalent circuit.

The filter of order $n=2$, from Table 2.1, the prototype elements values are

$$
\begin{aligned}
g_{1} & =1.4142 \\
g_{2} & =1.4142 \\
g_{3} & =1 \\
K_{12} & =1
\end{aligned}
$$


Now, $\alpha$ stay the same, and the new values for the coupling ratios are

$$
\begin{aligned}
& n_{1}=2.304 \\
& n_{2}=2.304
\end{aligned}
$$

Now, the elements of the input output coupling circuit will be performed and represented as stubs,

$$
\begin{aligned}
Y_{0} & =0.4886 \text { mhos } \\
Y_{01} & =0.5114 \text { mhos } \\
Y_{1} & =-0.3231 \text { mhos } \\
Y_{12} & =0.1174 \text { mhos } \\
Y_{r r} & =1.0808 \text { mhos }
\end{aligned}
$$

By using (2.191) and (2.228)

for $r=1$

$$
Y_{1}+Y_{01}+Y_{12}=1.0808 \text { mhos }
$$

for $r=2$

$$
Y_{2}+Y_{12}+Y_{23}=1.0808 \text { mhos }
$$

thus,

$$
Y_{1}=Y_{2}=0.6403 \text { mhos }
$$

The negative admittance in (2.330) has been absorbed in (2.335). The filter performance has been computed in the same procedure like that for the first order, the result of the calculated response is shown in Fig. 2.44. The two capacitor $C_{1}$ and $C_{2}$ are the tuning elements as shown in the equivalent circuit (Fig. 2.43), these two elements have to be identical for the best matching and the lowest insertion loss. A better selectivity has been gained by increasing the filter order, compared with the first order. Also the matching has been varied with less than $10 d B$ across the entire tuning range. The lower side attenuation has been improved because of the existence of a non-zero pole in the lower side of the frequency skirt.

To compute the static self and mutual capacitances between the parallel coupled lines, the admittance are converted to impedance and matched to $50 \Omega$, therefor 


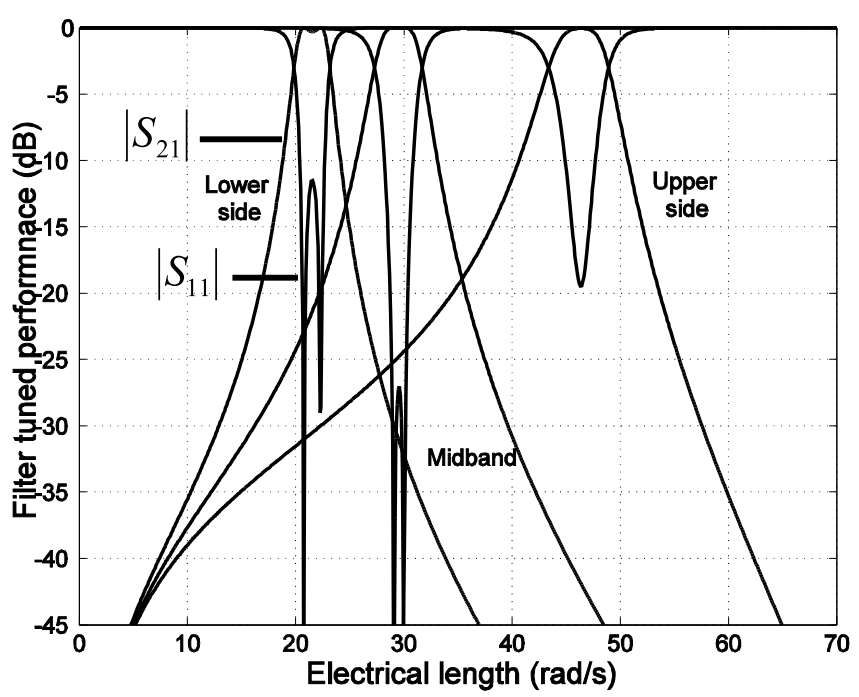

Figure 2.44.: Second order computed tuned filter performance, $\Delta \omega_{1}=\theta_{1} / a=171 \mathrm{MHz}$, thus, $\Delta \omega_{0}=394 \mathrm{MHz}$ and $\Delta \omega_{2}=744 \mathrm{MHz}$.

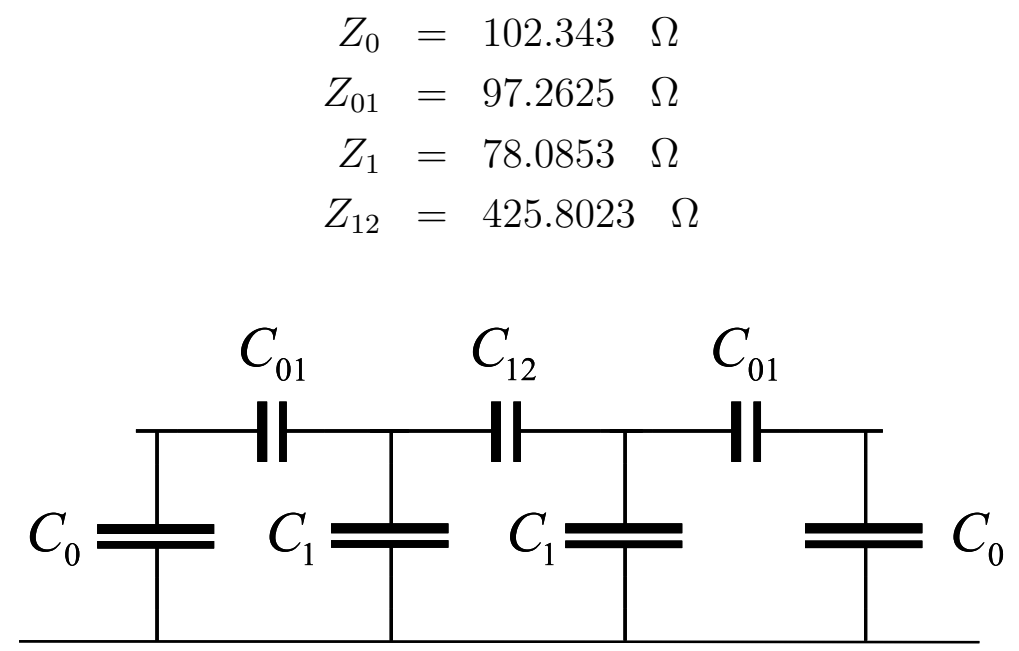

Figure 2.45.: self and mutual static capacitance definitions for the second order filter.

Hence

$$
\begin{aligned}
& \frac{\sqrt{\epsilon_{r}} C_{0}}{\epsilon}=3.6837 \\
& \frac{\sqrt{\epsilon_{r}} C_{01}}{\epsilon}=3.8563 \\
& \frac{\sqrt{\epsilon_{r}} C_{1}}{\epsilon}=4.8281 \\
& \frac{\sqrt{\epsilon_{r}} C_{12}}{\epsilon}=0.8854
\end{aligned}
$$


The definitions of these capacitances are shown in Fig. 2.45. Now, the two tuning capacitances have been deviated from each other by changing one of them from $0 \ldots 0.5 p F$. As the difference increased the matching is lost dramatically, a valley has been developed inside the insertion loss, which is concave up curve as well as the return loss, which is concave down. As the difference increase the the depth of the valley increase causing a two peaks on both curves. The coupling between the resonator is getting poor. Thus the shape of the filter is destroyed. Fig. 2.46 shows this.

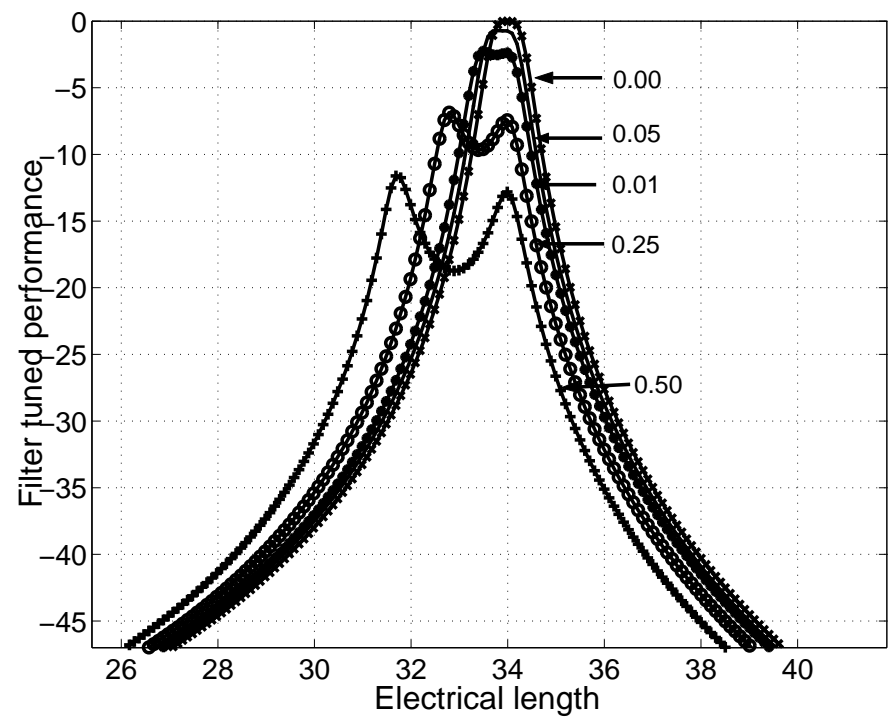

Figure 2.46.: Capacitances deviation, the numbers represent the difference $\Delta C$ between the two capacitors $C_{1}$ and $C_{2}$.

\subsection{Summary}

The fundamentals of tunable filters have been reviewed. The design procedure utilize the insertion loss synthesis techniques. Thus, starting from the specifications. Then, the elements value of the corresponding lowpass prototype are obtained. The selection of the filter parameters for the untuned state is chosen at the geometric means of the extremes value which corresponds to the lower and the upper tuning range. The value of the lumped tuning varactors and then the admittances of the filters are chosen. Finally the admittance of the input and output coupling elements are obtained. The admittance matric of the combline filter is then computed. The filter performance is calculated. Now the resulted filter could be build in different technologies as well as dimensions. Now, these capacitances depend on the geometrical structure as well as the substrate material properties. In this work, the filter will be build using the LTCC technology. Hence, the dimension will be adopted to that. 


\section{Technology}

This chapter treats the fabrication and assembly issues arising from the device structure which combines the two ceramics components, LTCC and piezoelectric actuator technologies.

The fabrication employs regular thick film LTCC processing of DuPont 951 tape with a permittivity of 7.8, a loss tangent of 0.002 and a sintered tape thickness of $100 \mu \mathrm{m}$. Filled vias of 150 $\mu \mathrm{m}$ diameter and $250 \mu \mathrm{m}$ patch, provide vertical through-contacts for second-level assembly. A $1 \times 1 \mathrm{~mm}^{2}$ cavity with ground metallization on its stepped side wall accepts conductive epoxy adhesive in the final assembly process of the piezoceramic part.

In [71] a PZT thick film has been cofired on LTCC substrate, the green LTCC 951 (DuPont) was used as a substrate. The LTCC/Silver/PZT has been cofired together, the electrical characteristics of this structure have deteriorated due to the interactions between the LTCC substrate and the PZT layer during the firing process. In order to guarantee optimum performance of both, the LTCC integrated coupled line filter elements and the piezoelectric element, at hybrid assembly process of two separately fabricated and optimized components is adopted here.

\subsection{Piezoelectric Bimorph Cantilever}

Piezoelectric bendable actuators are frequently used, when large displacements are required. They are used in a wide range of applications, particularly smart structures and systems, like shape-controlled radar and satellite antennas, and MEMS switches. In the present case, the bending actuator exhibits the additional favorable property that its fits into the filter structure without increasing its size excessively. As the central driver for the tuned capacitor, its basic performance principles will be summarized in the following from the data sheet of the manufacturer [72].

The model describing the deflection of a piezoelectric bimorph structure can be derived by applying basic mechanical principles of static equilibrium and strain continuity between successive layers in the device [73]. The deflection of the bimorph is found to be

$$
\delta(x, V)=3 d_{31}\left(\frac{x}{t}\right)^{2} V
$$

where $d_{31}$ is the piezoelectric charge constant, $V$ the voltage applied, $x$ the distance from the clamping point, and $t$ the thickness of a single piezoelectric layer. Accordingly, the tip of a cantilever of dimensions $L \times W \times T$ exhibits a displacement (according to Equation 3.1)

$$
d_{\text {air }}(V)=3 d_{31}\left(\frac{L}{T}\right)^{2} V
$$


which should ideally be just the air gap width.

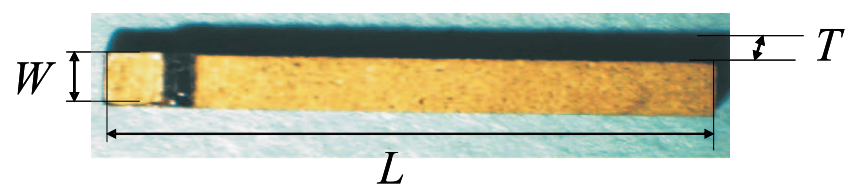

Figure 3.1.: Piezoelectric bimorph cantilever with dimension $7 \mathrm{~mm} \times 1 \mathrm{~mm} \times 0.48 \mathrm{~mm}$.

Fig. 3.1 shows the commercially available a piezoelectric bimorph cantilever employed for this work [74]. The material has a $d_{31}$ of $230 \mathrm{pm} / \mathrm{V}$, which results in a $50 \mu \mathrm{m}$ nominal deflection for $200 \mathrm{~V}$ bias.

The piezoelectric cantilever has a size of $7 \mathrm{~mm}$ in length, $1 \mathrm{~mm}$ in width, and $0.48 \mathrm{~mm}$ in thickness with composition of lead-zirconate-titanate (PZT) and has a bimorph structure. As shown in Fig. 3.2, the bimorph design comprises a gold electrode on top, a piezoelectric ceramic layer, an intermediate electrode, a second piezoelectric layer, and a gold electrode at the bottom. A fixed bias voltage of $200 \mathrm{~V}$ is applied between the top and bottom electrode parallel to the predefined polarization of the material. The drive or tuning voltage lies in between and is applied to the center electrode. By this, the lower and upper piezoelectric layers can be made to contract differently, resulting in a macroscopic bending of the bimorph structure. The bending is not always linear or uniform due to the characteristics of the material.

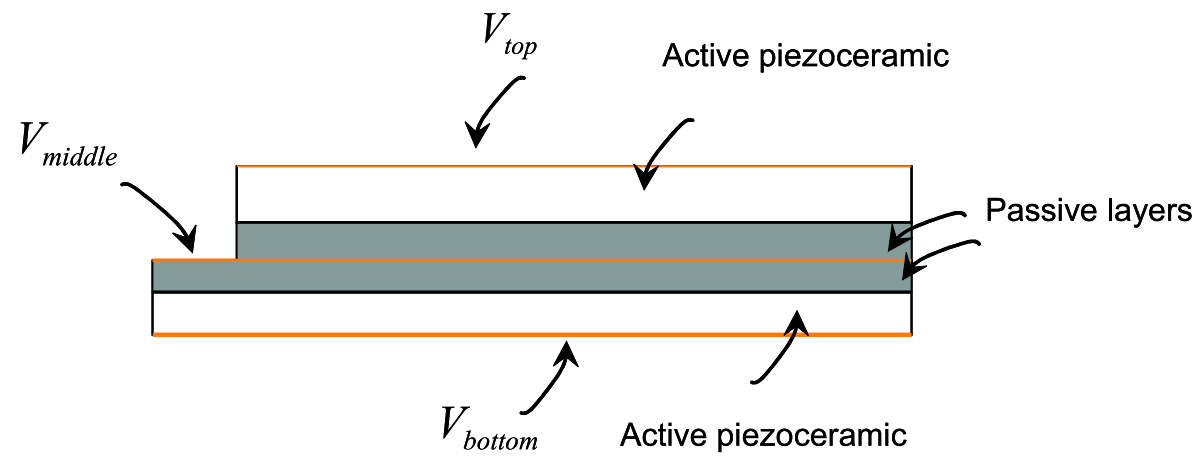

Figure 3.2.: Piezoelectric bimorph cantilever cross section.

Depolarization of the ceramics is avoided when operation is below $100^{\circ} \mathrm{C}$ i.e. with sufficient margin to the Curie temperature $200^{\circ} \mathrm{C}$ which describes the ferroelectric-to- paraelectric phase transition. The chemical composition of the piezoelectric material limits its operation for temperature, and voltage. Operating the PZT material outside of these limitations may cause partial or total depolarization of the material. Consequently, a diminishing or loss of the piezoelectric properties occurs. When the PZT is heated above the Curie temperature, all piezoelectric properties are lost. For a strong electric filed the PZT material can be depolarized with polarity opposite to the original poling voltage. Such a process will cause the ceramic to behave in unpredictable way which causes the relationship between the applied voltage and the displacement of the cantilever to change. Thus, the relationship between the applied voltage and the center frequency of the filter will change. For the present design care is also taken not 
to apply the bias in a direction opposed to the predefined polarization since the electric field (typically $>500 \mathrm{~V} / \mathrm{mm}$ ) would then destroy the performance even below Curie temperature.

\subsection{Standard LTCC Process}

LTCC is a multilayer ceramic process that can be used to fabricate low cost, high performance RF and microwave components. Its versatility is suited for a wide range of components from simple passive filter structures and packages to complex sub-system assemblies containing discrete SMT (Surface Mount Technology) components, bare die and printed passives. Excellent microwave properties of the dielectric glass ceramic tapes in conjunction with good conductor materials like silver, gold, palladium or platinum and thick-film resistors permit three-dimensionally embedded, hermetically sealed RF circuits of high integration density and small footprint. The ceramic layers are tape-cast in their pre-fired "green-state" and the tape is cut to the required size. Registration holes, via holes and cavities are then punched or drilled into the different tape layers. The via holes are normally filled, often with silver, and then

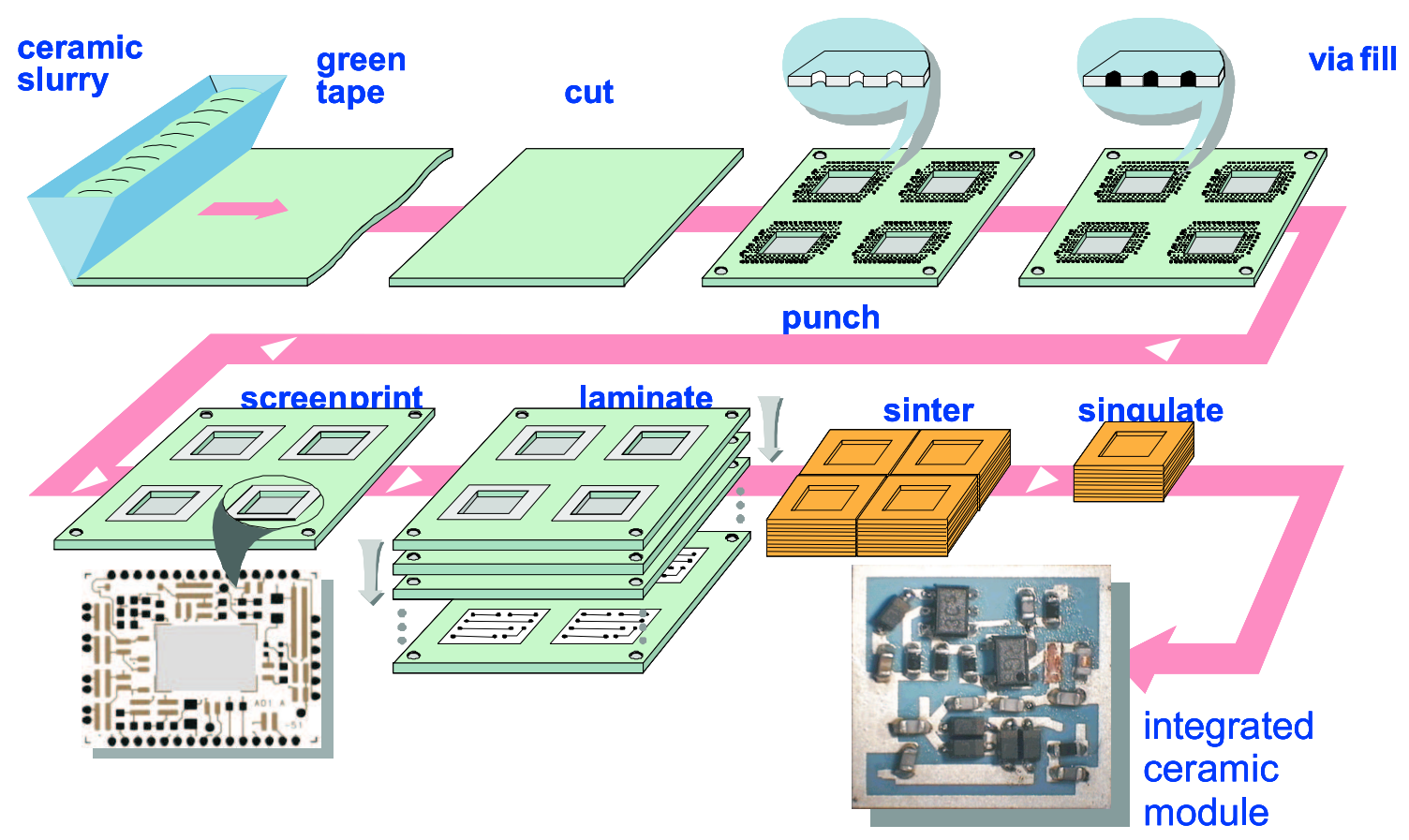

Figure 3.3.: LTCC process flow chart, Siemens CT MM2 Application Notes.

thick film processing is used to print metallization patterns on each, or selected tapes. When thick film processing is used, the minimum line width/gap is around $100 \mu \mathrm{m}$ [75]. The different layers are then inspected, registered and laminated and then co-fired at around $850^{\circ} \mathrm{C}$. Post fired processing of the top layer is also an option. A typical LTCC process flow chart is shown in Fig. 3.3. The assembly and interconnect technology between LTCC microstrip structures and piezoceramic element is important for the device performance. Control over the thin film 
air-gap capacitor on the thick film LTCC substrate requires the integration of a polishing step into the processing sequence.

\subsection{The Assembly Process}

Since the tunable capacitor is formed between the LTCC and the piezoelectric element, the assembly process of the two components is crucial. The following issues were particularly observed during the present development:

- A suitable two-component commercial epoxy [76] with sufficient processing time, low curing temperature, low shrinkage and good electrical conductivity is chosen.

- A special assembly tool was designed and used as shown in Fig. 3.4. The cantilever and the LTCC substrate are placed on a metal plate in correct relative position and fixed by a screw such, that they touch each other as close as possible. The plate is then turned upside down, now standing on its four legs.

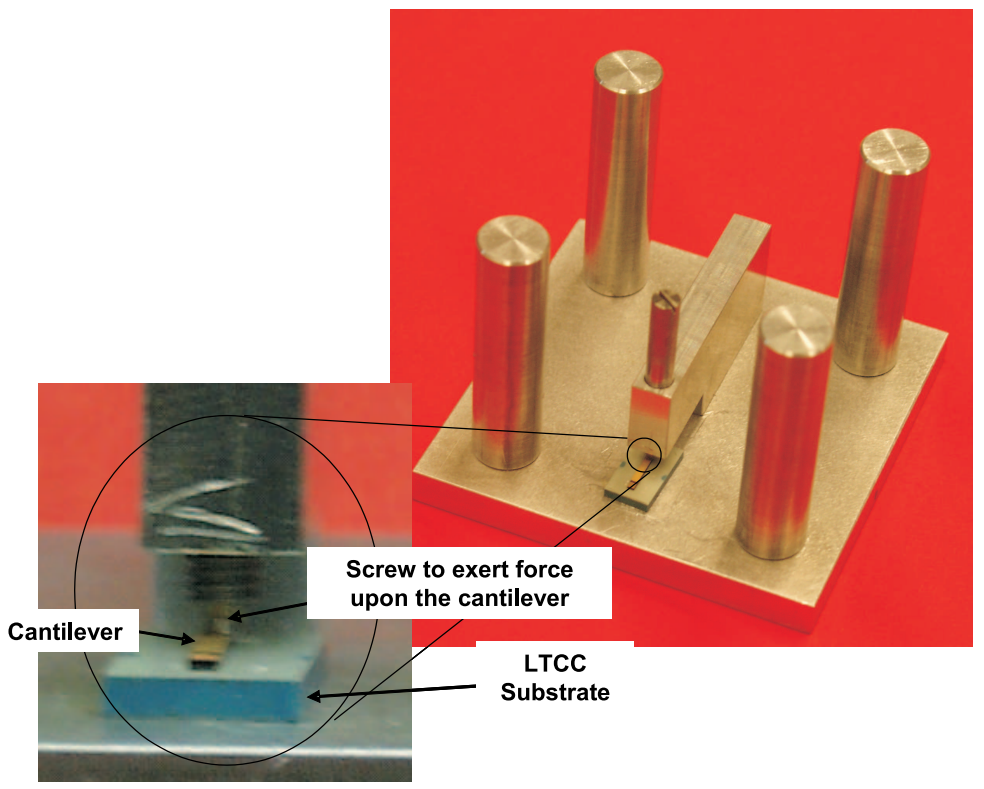

Figure 3.4.: Structure used to fix the cantilever to the LTCC substrate during the assembly process.

- The glue is subsequently injected through an opening into the LTCC cavity (Fig. 3.5). The amount is adjusted to just fill this cavity down to the electrode of the cantilever and assure a conductive connection of this end of the cantilever with the integrated LTCC electrode while the other end remains freely movable.

- Finally, curing the glue is done for $4 \mathrm{~h}$ at $85^{\circ} \mathrm{C}$. 


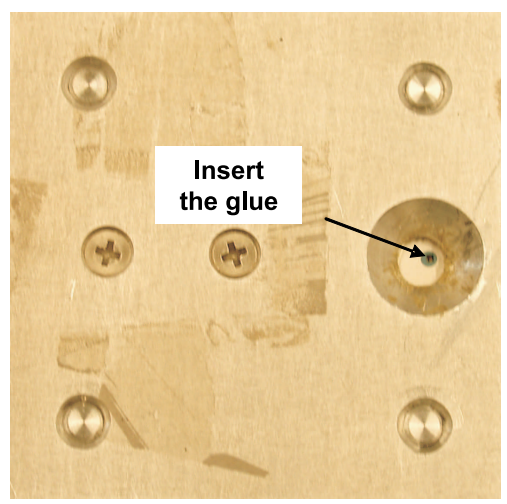

Figure 3.5.: Assembly process: glue.

\subsection{PZT Cantilever Characterization}

To measure the deflection, the cantilever and the LTCC substrate were glued together (see 4.6: "Device-Specific Fabrication Process"). The deflection was measured in an existing setup shown in Fig. 3.6 as a function of tuning voltage. It is based on Triangulation principle [77].

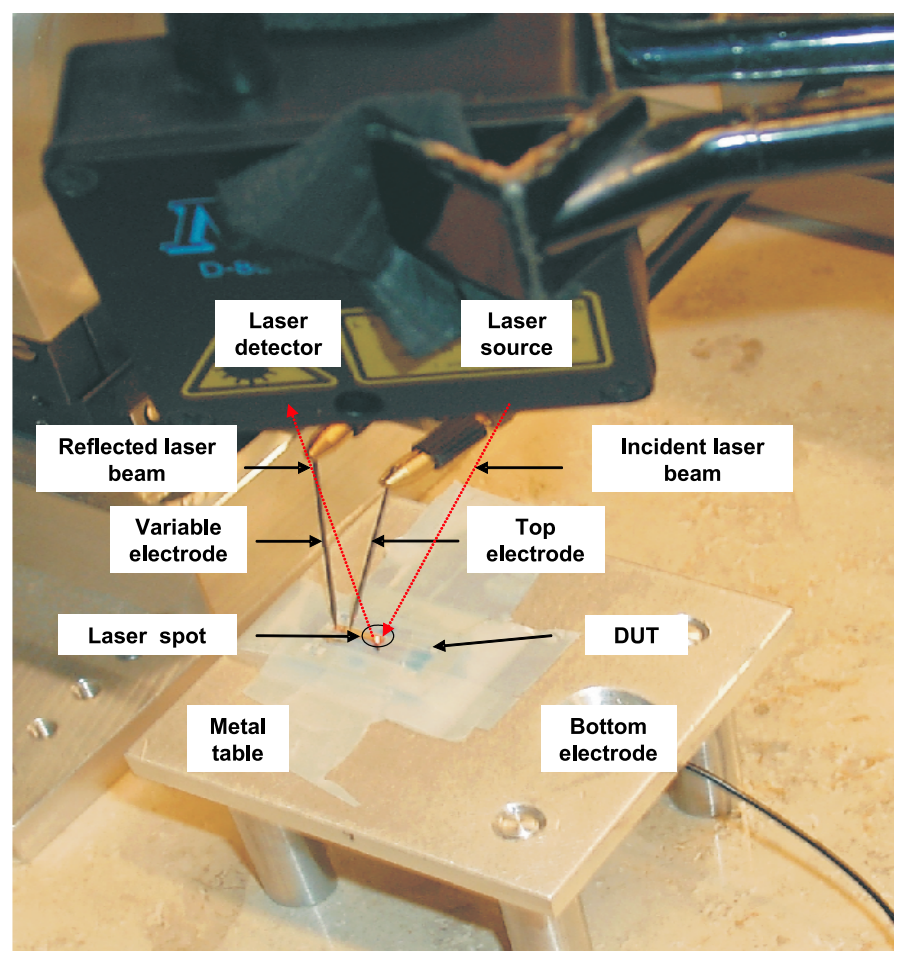

Figure 3.6.: Experiment Setup.

The device under test (DUT) has three electrodes: top, middle and bottom as described before. The top and the bottom electrodes were connected to a constant bias DC voltages of $200 \mathrm{~V}$ and $0 \mathrm{~V}$, respectively, while the middle one is connected to a variable voltage source, which can be 
varied from 0 to $200 \mathrm{~V}$. The voltages at both the top and the middle were applied by using of DC needles. The bottom electrode is formed by the bottom metallization of the LTCC substrate making contact to the chuck (table carrying the sample). A laser optical source creates a light

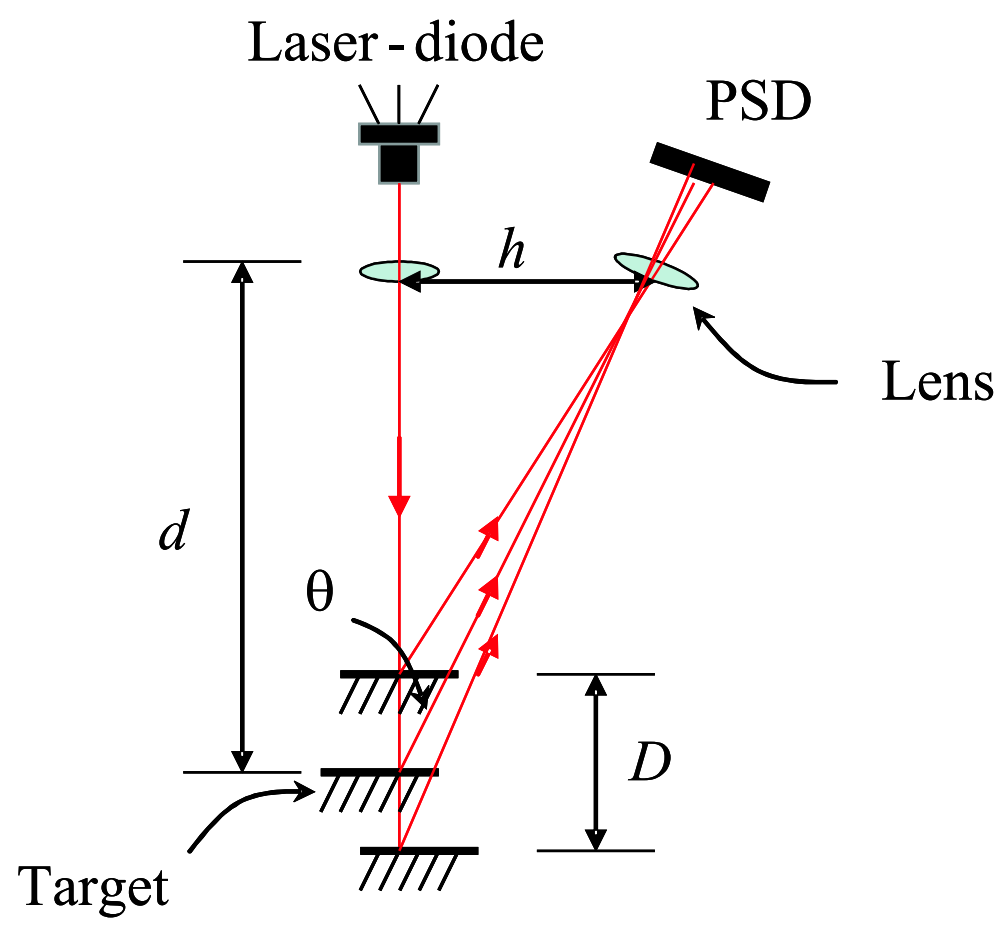

Figure 3.7.: Triangulation calculation.

spot on the cantilever tip which is observed by a position sensitive device (photodiode array, PSD) under an oblique angle (Fig. 3.7). The PSD element supplies a position dependent, analog output voltage proportional to the geometric shift. A diffuse reflection is necessary for the triangulation principle. A calibration is done first to see if there is a target in the measuring range and if the amount of light is sufficient for the PSD. The measurement accuracy can be affected by the angular relationship of the sensor to the target surface.

The distance between the laser source and the PSD detector is $h . d$ represents the reference distance that result from the calibration. From the self test, both $d_{0}$ and $\theta_{0}$ are known for the initial state. A deflection, $D$, of the cantilever causes $d_{0}$ and $\theta_{0}$ to change into $d$ and $\theta$ from which $D$ can be calculated according to

$$
D=\frac{h \sin \left(\theta-\theta_{0}\right)}{\sin (\theta) \sin \left(\theta_{0}\right)}
$$

positive values correspond to upward, negative to downward deflection 


\subsection{Measurements and Analysis}

The voltage pulse which is shown in Fig. 3.8(a) has been applied to the middle electrode of the cantilever. The applied voltage has four regimes, the first, increasing the voltage from 0 to 200 $\mathrm{V}$ over $3.5 \mathrm{~s}$, the voltage has been kept constant at $200 \mathrm{~V}$ for $2 \mathrm{~s}$ in the second regime. It has been decreased form $200 \mathrm{~V}$ back to $0 \mathrm{~V}$ over $3.5 \mathrm{~s}$ in the third one and finally it kept at $0 \mathrm{~V}$ over 6 s. At zero bias, a zero difference voltage exists between the middle electrode and the bottom

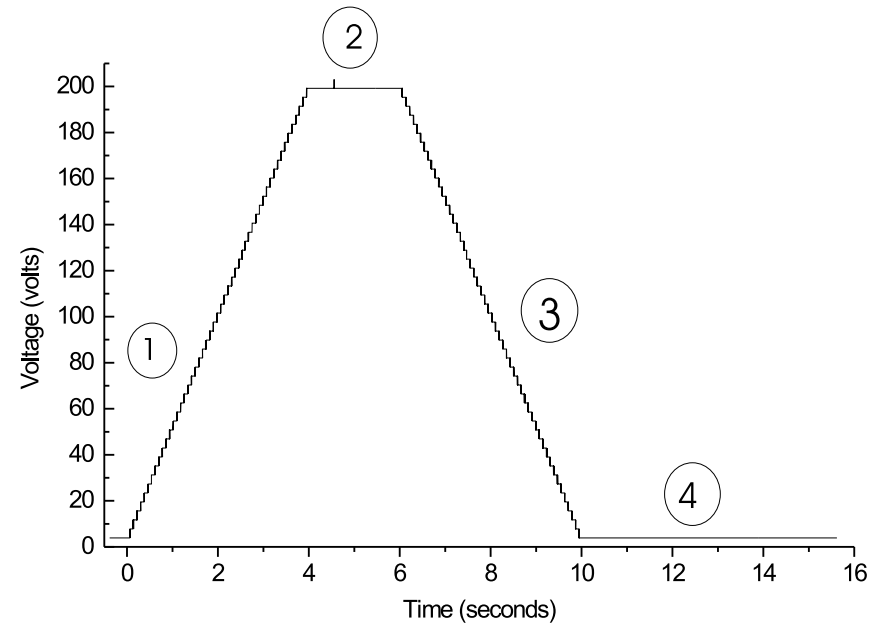

(a) The applied voltage pulse versus time.

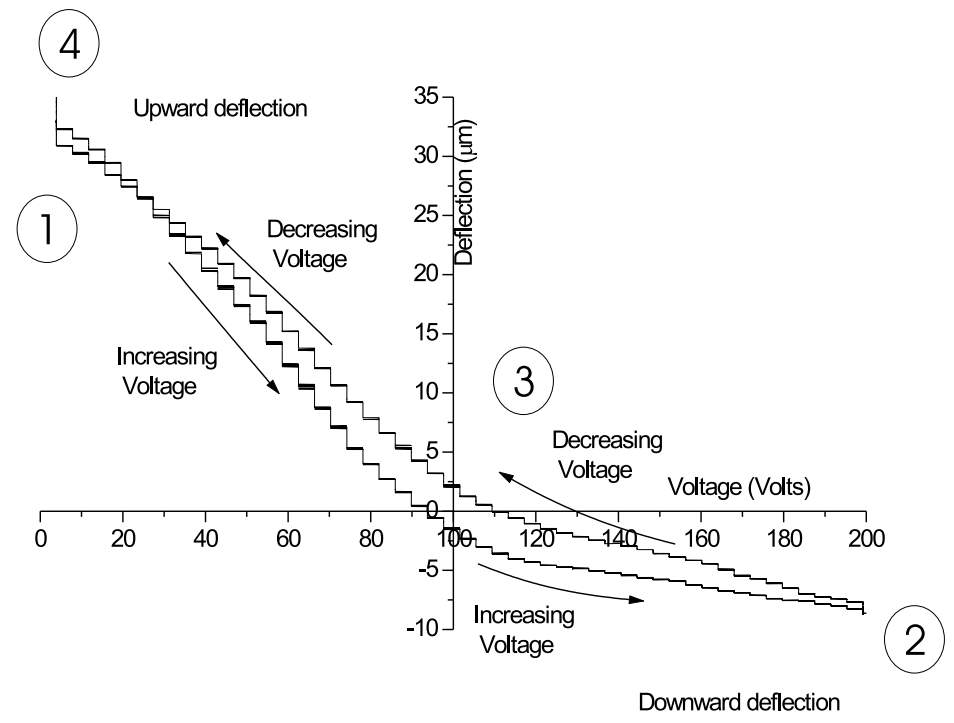

(b) Mechanical deflection measurements versus voltage.

Figure 3.8.: Characterization of piezoelectric actuator glued to LTCC substrate 
electrode and of $200 \mathrm{~V}$ between the middle electrode and the top electrode which causes the cantilever to deflect maximally upwards away from the LTCC substrate. When the voltage is increased and reaches $100 \mathrm{~V}$, the electric field is equal in both layers and no deflection results. Finally, towards 200V, the role of the two layers is reversed and the cantilever bends down. This behaviour is reflected by the measurement in Fig. 3.8(b). Since the electric field causes both, a polarization and a contraction of the material, the deflection is subject to hysteresis. Furthermore, there is asymmetry between up and down movement, which is attributed to the existence of the substrate and the glue on the bottom side. After bending $8 \mu \mathrm{m}$ down the cantilever is blocked on this sample by the substrate. The finite extension of the glue also limits the maximum upward deflection to $32 \mu \mathrm{m}$. 


\section{Design of Tunable LTCC Bandpass Filters}

The design for a piezoelectrically driven variable capacitor with wide tuning range is presented. The proposed capacitor is tuned by varying the gap width between the electrodes. In contrast to the conventional two parallel plate capacitors, the present approach employs a piezoceramic cantilever to move the top electrode. This varactor can be further integrated with passive components to yield specific frequency-tunable characteristics.

\subsection{Principle of Operation}

In this section I present the operating principle of the device using a specifically designed and fabricated low temperature cofired ceramics (LTCC) substrate to which a commercial piezoelectric actuator is mounted. A schematic cross-sectional view is shown in Fig. 4.1. Similar to the known micro-electromechanical (MEMS) approaches for tuning and switching, a modified parallel plate capacitor with a solid state high-permittivity dielectric layer and piezoelectrically movable top-electrode is proposed as a promising and versatile tuning element. Similar to MEMS devices, the capacitance variation is due to the movement of the electrodes and not due to the change in material parameters such as paraelectric material in BST varactor. This component therefore has the potential for a high quality factor. The vertical movement of the

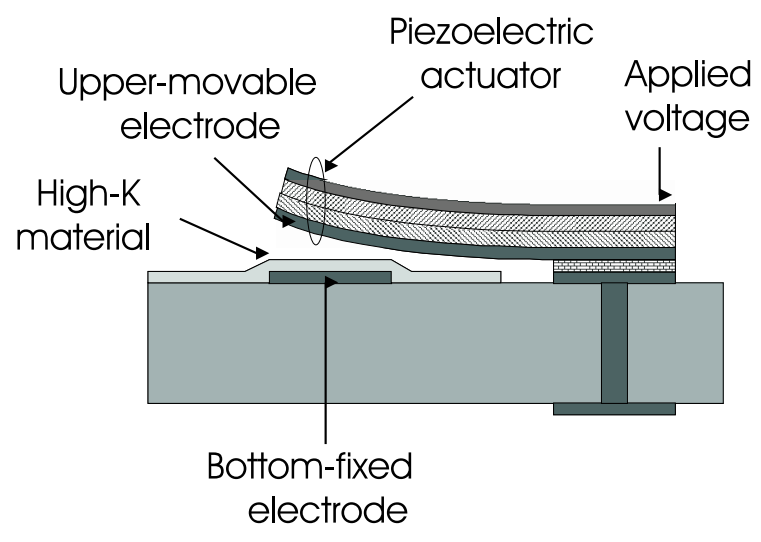

Figure 4.1.: Schematic of the piezoelectric LTCC varactor.

top electrode opens an air gap which together with the high-K (an insulating material with a high dielectric constant) film forms an effective dielectric material between the electrodes. 
For sufficiently different dielectric properties of the film and the air gap the capacitance of the device can be tuned in a wide range even for small electrode movements.

In MEMS capacitors the bottom electrode is often covered by a dielectric layer with a thickness of 100-200 nm and a relative low dielectric constant between 3 to 8 to prevent a short circuit between the top and the bottom plates. In this approach, the high-K layer is of thickness $10 \mu \mathrm{m}$ and a high dielectric constant of 65 achieve a sufficiently strong capacitance change upon opening of the air gap.

\subsection{Design}

The capacitor design must consider the following figures-of-merit: frequency of operation, self-resonance frequency, maximum-to-minimum capacitance ratio and quality factor. The specifications depend on the intended application of the variable capacitor. The motivating application of the research is the tunable capacitor for RF voltage-controlled oscillator, tunable bandpass filter, matching circuits. The self-resonance frequency (SRF) for these applications should be at least twice the desired operating frequency. The design approach of a high- $Q$ tunable capacitor must focus on the thickness of the high-K film and the control of the air gap. In zero-bias state, the air gap width becomes theoretically zero and the capacitance assumes its maximum value. In this case, the electrodes are separated by a high-K dielectric layer. Only with bias, the upper electrode is raised and the capacitance decreases due to the air gap. The approximate capacitance value is calculated by the parallel plate formula [78]

$$
\begin{aligned}
C & =\frac{\epsilon_{0} \epsilon_{r e} A}{d} \\
\frac{d}{\epsilon_{r e}} & =d_{\text {air }}+\frac{d_{K}}{\epsilon_{K}} \\
d & =d_{\text {air }}+d_{K} \\
d_{\text {air }} & =3 d_{31}\left(\frac{L}{T}\right)^{2} V .
\end{aligned}
$$

where

$\epsilon_{0}$ : the vacuum permittivity of $8.85 \times 10^{-12}(\mathrm{pF} / \mathrm{m})$.

$\epsilon_{r e}:$ the effective permittivity of the air plus the high-K dielectric layer.

$A$ : the area of the capacitor.

$d_{\text {air }}$ : the effective air gap inside the capacitor at the tip of the cantilever.

$d_{K}$ : the thickness of the high-K dielectric layer.

$\epsilon_{K}$ : the effective permittivity of the high-K dielectric layer.

$d_{31}$ : the piezoelectric constant of the material of $230 \mathrm{pm} / \mathrm{V}$.

$V$ : the actuation applied voltage.

$L$ : the length of the piezoelectric cantilever of $7 \mathrm{~mm}$.

$T$ : the thickness the piezoelectric cantilever of $0.13 \mathrm{~mm}$.

The tuned capacitor varies between its maximum value as determined by the electrode area as 
well as the thickness and dielectric constant of the high-k material, and its minimum value as determined by a larger air gap $\left(d_{\text {air }} \gg d_{K}\right)$. So we have

$$
\frac{\epsilon_{0} A}{\left(d_{K}+d_{\text {air }}\right)} \leq C \leq \frac{\epsilon_{0} \epsilon_{K} A}{d_{K}} \Rightarrow \frac{C_{\max }}{C_{\min }}=\frac{\left(d_{K}+d_{\text {air }}\right) \epsilon_{K}}{d_{K}}
$$

Fig. 4.2 demonstrates the wide tuning range of the capacitance for the case of a $10 \mu \mathrm{m}$ thick dielectric layer with a permittivity of 65 between 2 and $60 \mathrm{pF} / \mathrm{mm}^{2}$.

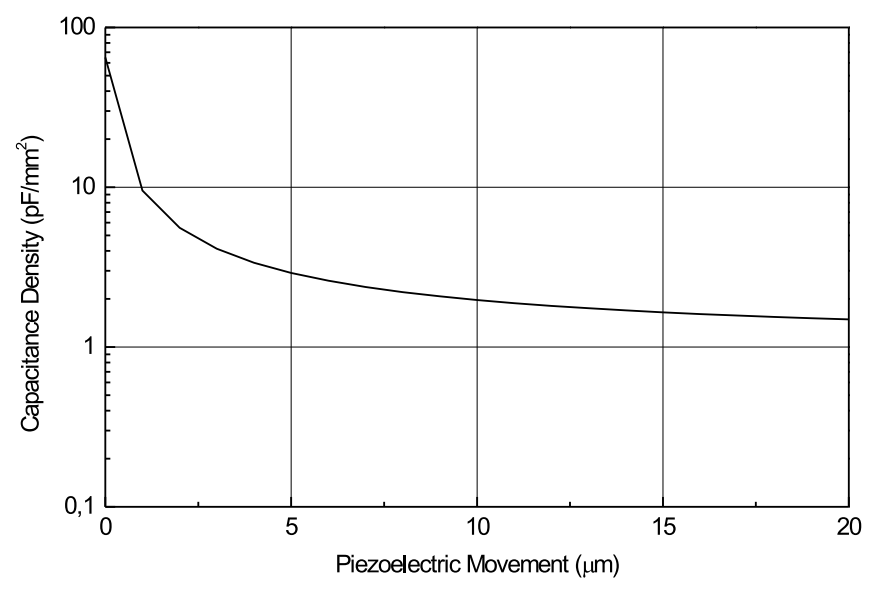

Figure 4.2.: Tuning of a multilayer capacitor by adjusting the width of the air gap.

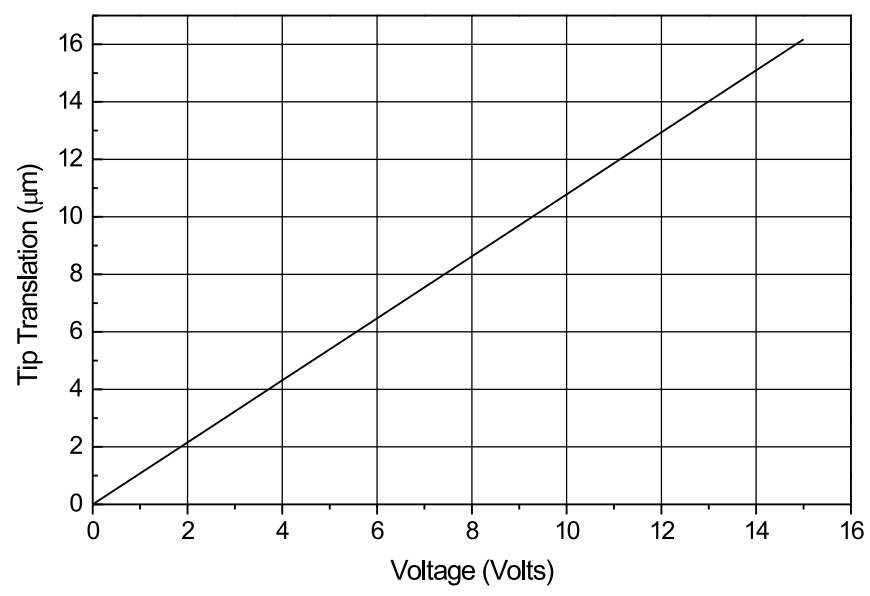

Figure 4.3.: Typical cantilever deflection of a commercially available piezoelectric actuator. 
As a part of this approach, it is suggested that the movement is realized by a piezoelectric actuator which is capable of moving the electrode accurately on a micrometer scale. A typical case $\left(d_{31}=0.23 \mathrm{~nm} / \mathrm{V}\right.$, free effective-length $L$ of $6 \mathrm{~mm}$ and PZT thickness of $T$ of $\left.0.28 \mathrm{~mm}\right)$ is shown in Fig. 4.3. Combining Figs. 4.2 and 4.3 it can be observed that a few volts are sufficient to tune the capacitance by a factor of 43 . This is about an order of magnitude better than in the case of traditional semiconducting or paraelectric varactors or other MEMS varactors.

\subsection{Tunable Bandpass Filter}

With a tunable circuit, the tuning can be accomplished by varying either the length, the inductive or capacitive loading of the transmission line. When the value of a capacitor in a circuit is changed, the impedance and phase relationships in this circuit affected in predictable ways. This change can be exploited to yield an analogue active or passive front end components with wide bandwidth or tuning range, respectively. A microstrip combline filter structure has been chosen as a demonstrator, Fig. 4.4. One of the two sections of the center line is identical

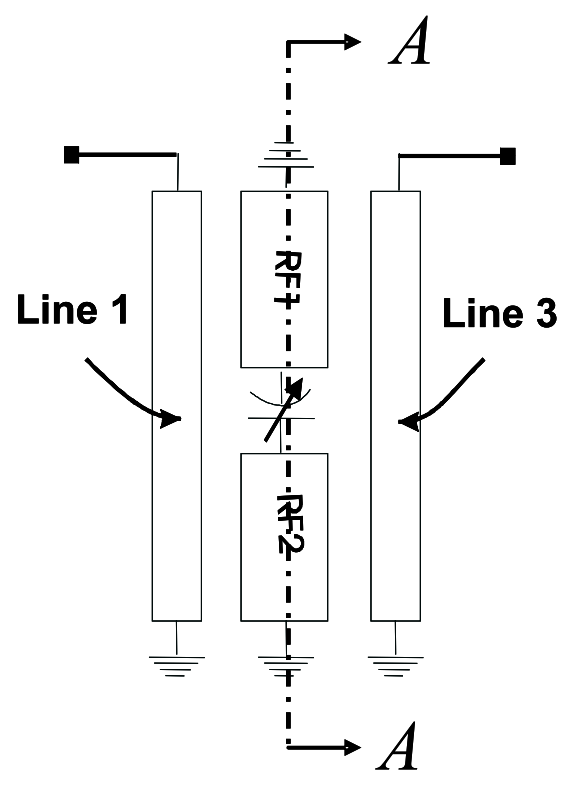

Figure 4.4.: One--pole microstrip combline bandpass filter.

with the bottom metallization of the piezoelectric cantilever ( $\mathrm{RF} 1$ ), which is shortened to ground at one end, and on the other end it forms the upper-movable electrode of the variable capacitor. The second section of the line (RF2) is on the LTCC substrate (see Fig. 4.5), thus forming the bottom-fixed electrode of the capacitor. The variable capacitor is formed by the overlapping area of the two lines (RF1 \& RF2).

By splitting the center line into two sections and inserting the capacitor at the middle of this line, the following advantages are gained:

1. Elimination of the cantilever inductance. 


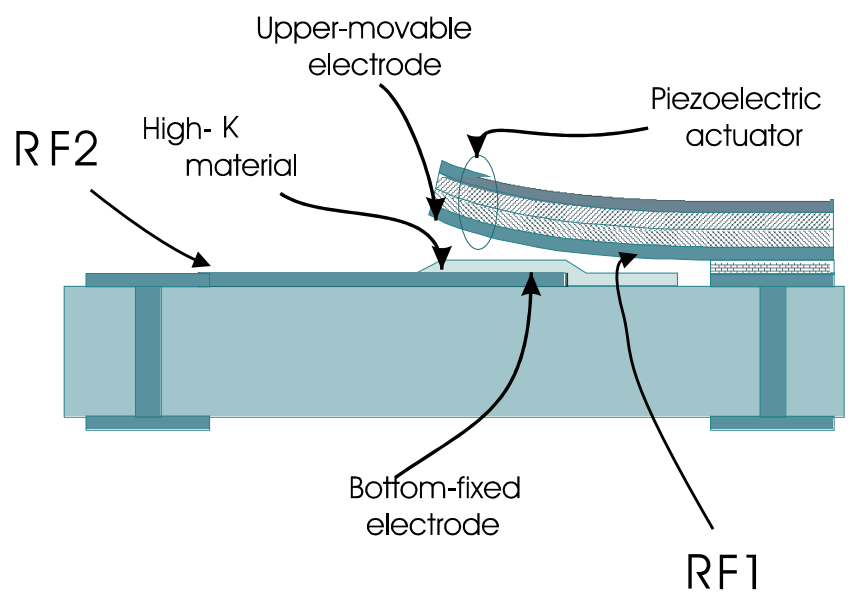

Figure 4.5.: Schematic of the tunable piezoelectric LTCC combline filter.

2. Infinite self-resonance frequency.

3. Expansion the frequency of operation.

4. Increasing of the quality factor.

5. Maintenance of a compact size, no extra space is required for the tuning function.

6. Reduction of the losses in the device that introduced by the metallization of the bottom side of the long piezo electric actuator.

7. Expansion of the tuning range.

\subsection{Filter Synthesis Design}

Since the width of the interior lines is predefined, because it is commercial available and not customized to this application, actually it is used for hearing-aid and not for tunable filter. The filter dimension will be adopted accordingly. The filter shall be realized on LTCC substrate of permittivity 7.8 , loss tangent 0.002 , and $1.2 \mathrm{~mm}$ thickness. The proposed filter schematic is shown in Fig. 4.4, and its cross-section is shown is Fig. 4.6. Since the predefined width is 1 $\mathrm{mm}$, which is equal to the width of the center coupled line, hence the shape ratio $W / H$ is equal to $1 / 1.2=0.8333$. Wheeler's [79] synthesis formula may be used to calculate the internal filter impedance $Z_{0}$.

$$
\begin{gathered}
\left(\frac{W}{H}\right)_{s}=(2 / \pi)(d-1)-(2 / \pi) \ln (2 d-1)+\frac{\left(\epsilon_{r}-1\right)}{\pi \epsilon_{r}}\left(\ln (d-1)+0.293-\frac{0.517}{\epsilon_{r}}\right) \\
d=\frac{60 \pi^{2}}{Z_{0} \sqrt{\epsilon}_{r}}
\end{gathered}
$$




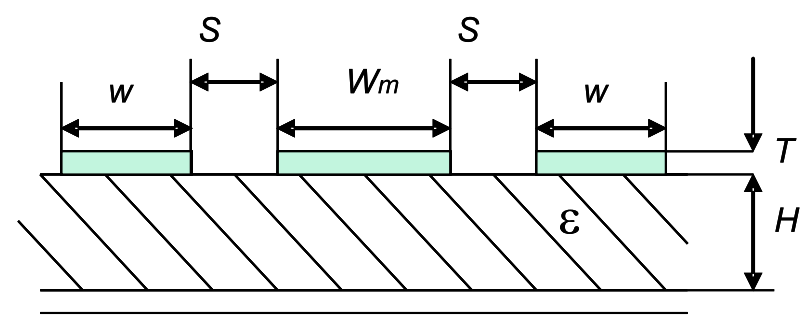

Figure 4.6.: Designed filter cross section, $w$ is the width of the input/out put lines, $T$ is the metallization thickness, $H$ is the thickness of the substrate, $\epsilon$ is the dielectric constant of the substrate, $S$ is the spacing between the lines, and $w_{m}$ is the width of the internal line, it is predefined by the width of the cantilever

Now, solving (4.6) for $Z_{0}$ give $70 \Omega$.

The self- and mutual capacitance per length have been previously computed, they are: $C_{0}$ $=4.2973, C_{1}=5.9110$, and $C_{01}=3.2427$, these values are normalized with the free space permittivity and scaled by $\sqrt{\epsilon_{r}}$. The normalized coupling coefficients $M_{i j}$, in terms of these coefficients could be found as follow [80]:

$$
M_{i j}=\frac{C_{i j}}{\sqrt{C_{i} C_{j}}}
$$

Therefore, $M_{01}$ is the coupling between the input/output line and the internal line, which is equal to 0.643. The determination of the spacing is done as follows: Figure 9.11 on [80] shows the measured coupling coefficients as a function of the $S / H$ for a substrate of 9.8 dielectric constant. This figure is used to find the corresponds value to the computed $M_{01}$ which is 0.19 . Another possibility is to used Milligan algorithm [81]. Hence, the spacing $S$ is $0.25 \mathrm{~mm}$. Now, The length of the resonator is $32^{\circ}$ at $1.69 \mathrm{GHz}$. The effect of the via hole ground on frequency shift has to be taken into account for determining the actual length. The actual length is 11.45 $\mathrm{mm}$ (calculate the theoretical length and subtract the via length). Finally, the width of input and output line has been chosen to be $0.5 \mathrm{~mm}$. Table 4.1 summarize all filter dimensions.

Table 4.1.: Filter physical dimensions

\begin{tabular}{|l||l|}
\hline Widths of lines the input \& output $(\mathrm{w})$ & $0.5 \mathrm{~mm}$ \\
\hline Width of the center coupled line $\left(W_{m}\right)$ & $1.0 \mathrm{~mm}$ \\
\hline Spacing between the lines $(\mathrm{S})$ & $0.25 \mathrm{~mm}$ \\
\hline Lengths of lines $(\mathrm{l})$ & $11.4 \mathrm{~mm}$ \\
\hline
\end{tabular}




\subsection{Device Simulation}

Like MEMS, the computational analysis of piezoelectrically tunable microwave filter (PTF) poses distinctive challenges, particularly for the modelling the thin air gap of only a few micrometers height but large lateral extension. An integrated variable air gap piezoelectric filter consists of both mechanical and electronic parts. Since the bottom metallization of the actuator has been utilized as a part of the device, its parabolic bending will create a nonuniform air gap that varies from zero at the clamping point to several microns at the tip which result in very high aspect ratios and inadequately large if not prohibitive number of cells for correct simulations. The time required to simulate these large simulation can be. As a solution, the bottom side of the cantilever is replaced by a straight line while the air gap inside the structure is replaced by an effective uniform layer.

\subsubsection{Simulation Tools}

The most popular techniques with a wide range of applications are Finite Difference Method [82], Finite Element Method [83], Transmission Line Matrix Method [84], and Method of Moments [85] Technique. The schematic representation of the structure is shown in Fig. 4.7(a). Sonnet em is a full-wave simulation tool. Since it was available for the present work, it has been used to simulate the PTF. Its method-of-moments based electromagnetic simulator solving the current distribution on 3D and multilayer structures of mostly planar structures like microstrip and striplines. It has been widely used in the design of LTCC circuits, microwave/millimeterwave circuits. Sonnet simulations of the PTF yield good agreement with the measurements when the model in Fig. 4.7(b) is used.

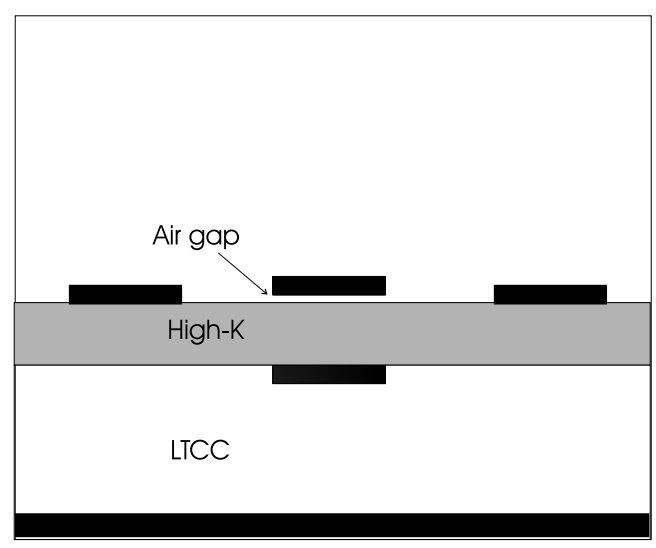

(a) Structure cross-section.

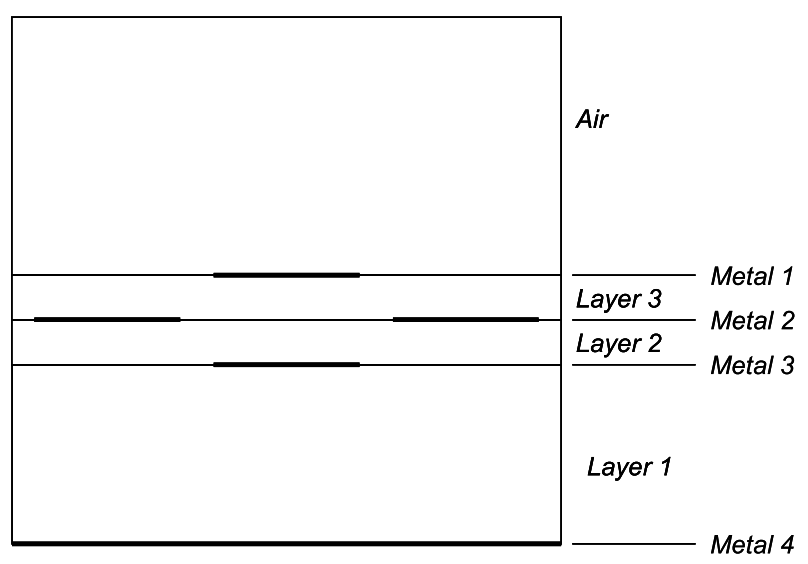

(b) Sonnet model.

Figure 4.7.: Simulation model

The specifications of the dielectric layers and of the metallization are shown in Table. 4.2 and Table. 4.3, respectively. Layer 3 represents the air gap. By changing the thickness of this layer, the effect of the tuned air gap is modelled. 
Table 4.2.: Dielectric layers.

\begin{tabular}{|l|l|l|l|}
\hline & Layer 1 & Layer 2 & Layer 3 \\
\hline Thickness $(\mu \mathrm{m})$ & variable & 10 & 1000 \\
\hline Material & Air & High-k & LTCC \\
\hline Permittivity & 1 & 65 & 7.8 \\
\hline Dielectric loss tangent & 0 & 0.002 & 0.002 \\
\hline
\end{tabular}

Table 4.3.: Metal types.

\begin{tabular}{|l|l|l|l|}
\hline & Thickness & conductivity $(S / \mathrm{m})$ & Material \\
\hline Metal 1 & $80(\mathrm{~nm})$ & $4 \times 10^{7}$ & gold \\
\hline Metal 2 & $10(\mu \mathrm{m})$ & $4 \times 10^{7}$ & silver \\
\hline Metal 3 & $10(\mu \mathrm{m})$ & $4 \times 10^{7}$ & silver \\
\hline Metal 4 & $10(\mu \mathrm{m})$ & $4 \times 10^{7}$ & silver \\
\hline
\end{tabular}

The first order combline filter designed in the pervious section has been simulated by sonnet em (Fig. 4.8).

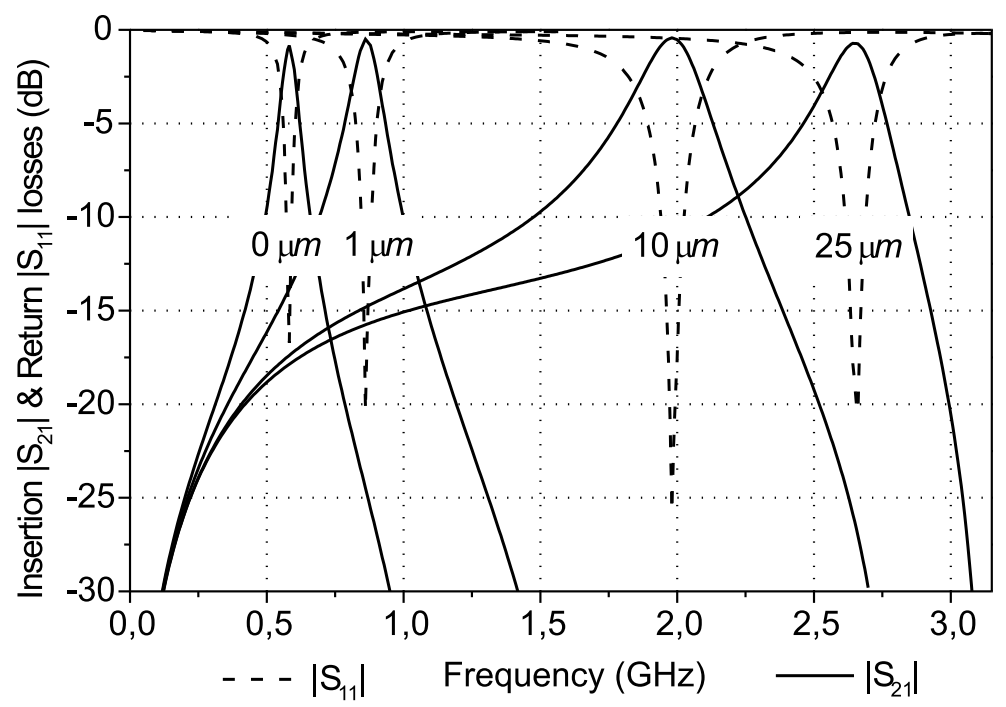

Figure 4.8.: Simulated tuning of the bandpass filter by the variable capacitor.

Small air gaps cause large frequency shift. Therefor a smooth surface is required. To achieve this, the fabrication has to fulfil requirements in substrate polishing and cantilever assembly. The minimum frequency at zero bias will otherwise be shifted up, thus reducing the tuning range. 


\subsection{Device-Specific Fabrication Process}

The fixed lower electrode of the tunable capacitor is printed on top of the $1.2 \mathrm{~mm}$ thick substrate (Fig. 4.9a). Proprietary high-K tape is used to provide the highly dielectric layer between the capacitor electrodes (Fig. 4.9b). To balance shrinkage and thermal stress during sintering, a symmetric stack of tapes is added on top, which also provides sacrificial material for the polishing step (Fig. 4.9c). Subsequently the bottom surface is slightly polished for the planarity of the top surface. The sacrificial material at the top is removed completely, and the polishing process is proceeded until the desired thickness of the high- $\mathrm{K}$ tape $(8 \mu \mathrm{m}$, permittivity 65 , loss tangent 0.002 ) and a smooth flat surface is reached (Fig. $4.9 \mathrm{~d}$ ). The microstrip coupled lines 1 $\& 3(\mathrm{w}=0.5 \mathrm{~mm}, \mathrm{~s}=0.25 \mathrm{~mm}, \mathrm{~L}=11 \mathrm{~mm})$ and the ground metallization are screen printed on both top and bottom surfaces. The movable part of the center line (RF1) is identical with the cantilever bottom metal ( $\mathrm{w}=1 \mathrm{~mm}, \mathrm{~L}=7 \mathrm{~mm}$ ). It overlaps with the thick-film section (RF2) on the LTCC substrate to form a capacitor area of $1 \times 1 \mathrm{~mm}^{2}$ (Fig. $4.9 \mathrm{e}$ ).

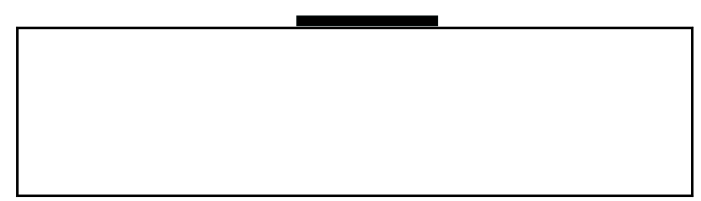

(a)

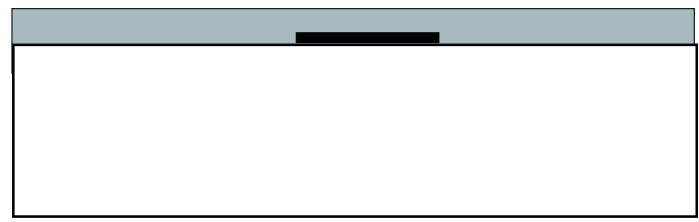

(b)

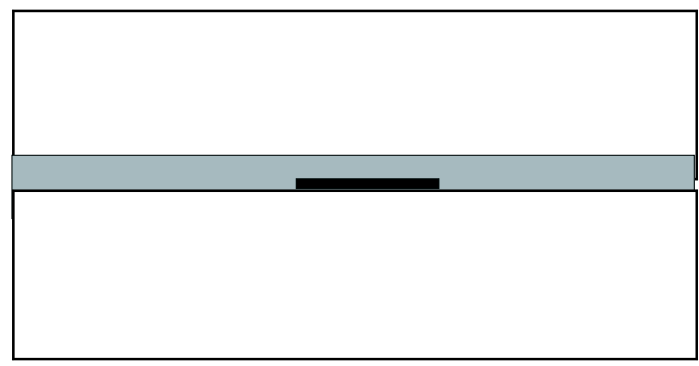

(c)

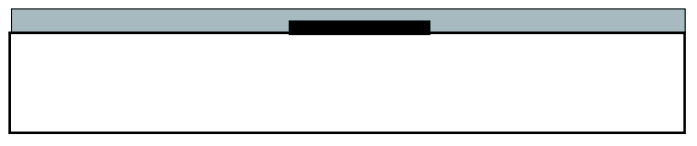

(d)

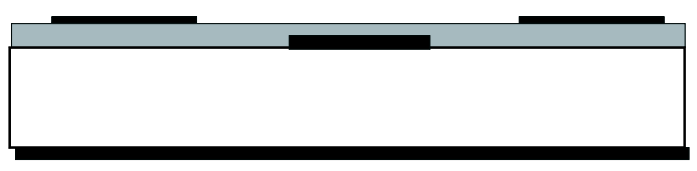

(e)

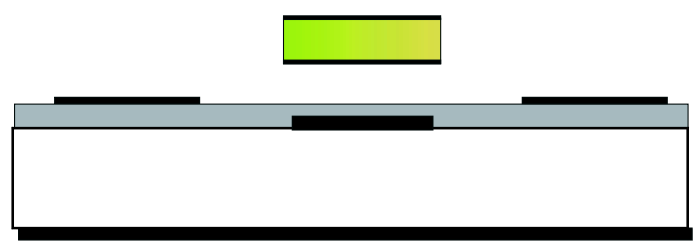

(f)

Figure 4.9.: Fabrication process. 


\subsubsection{The Fabricated Device}

Fig. 4.10 shows a photograph of the final device. Above the high-K tape, the input and the output lines $(1 \& 2)$ ended with via pads from one-end and on the other-end form the input/output GSG pads. The design uses $800 \mu \mathrm{m}$ GSG pads. The bottom electrode of the used piezoelectric actuator has been used as one of center coupled line sections (RF1). It is shown that one-end of the cantilever is fixed to the LTCC substrate by glue, and the other one left free. The other section of the center coupled line (RF2) is embedded between the high-K tape and the LTCC substrate, it is invisible. The variable capacitor is formed by the overlapping area between the sections of the center line. The angular misalignments between the fixed lines $(1 \& 2)$ and RF2 with respect to the cantilever electrode (RF1) could be noticed. The overall size is approximately $12 \times 7 \mathrm{~mm}^{2}$.

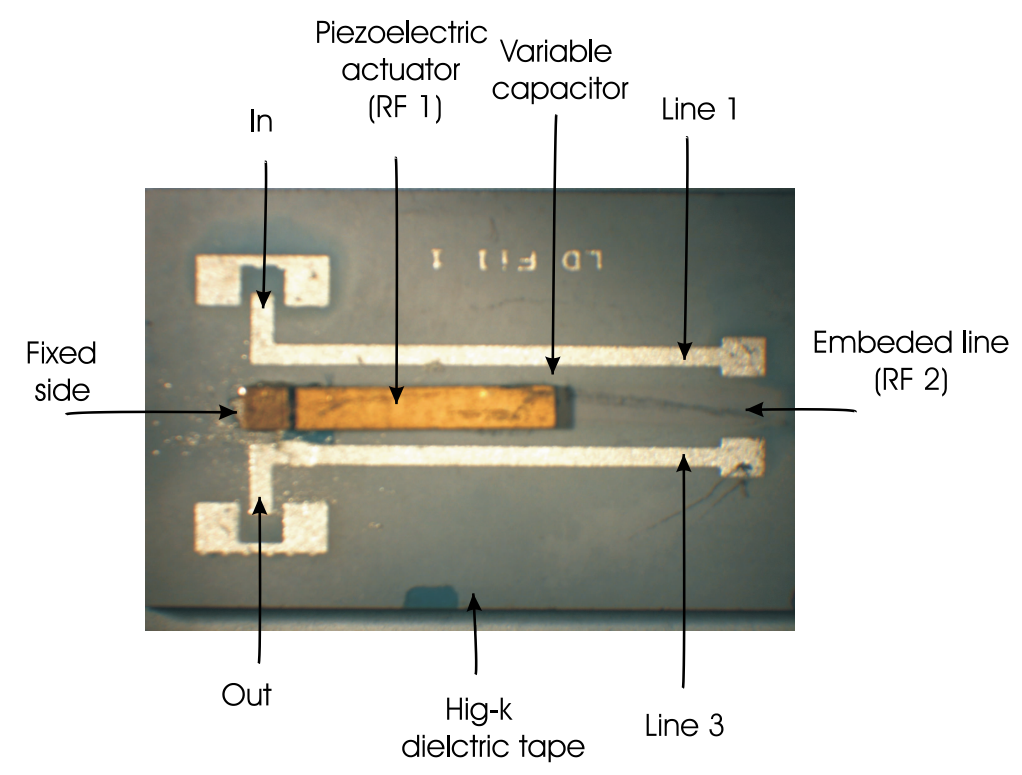

Figure 4.10.: The fabricated tunable bandpass filter.

\subsection{RF Measurements}

After the measurement set-up briefly explained, experimental results for the filter are presented. The center frequency of the filter is tuned from $1.1 \mathrm{GHz}$ to $2.6 \mathrm{GHz}$ with $200 \mathrm{~V}$ control voltage and low insertion loss of value $4 \mathrm{~dB}$ (at zero-bias) to $2 \mathrm{~dB}$ (at the maximum-bias). The resulting continuous decrease in capacitance causes a large shift of the center frequency by 1.5 GHz of the bandpass filter. The present filter design focuses on the demonstration of feasibility, tuning range, and device compactness. Although no optimization was done for power consumption, tuning speed, drive voltage, filter attenuation, and insertion loss, the approach already demonstrates low insertion loss due to the absence of lossy materials. This chapter also shows the effects of tuning mechanism on the overall quality factor, return loss, insertion loss, 
and the relative bandwidth at the mid of the band as a function of frequency across the entire tuning range.

\subsubsection{Measurements Setup}

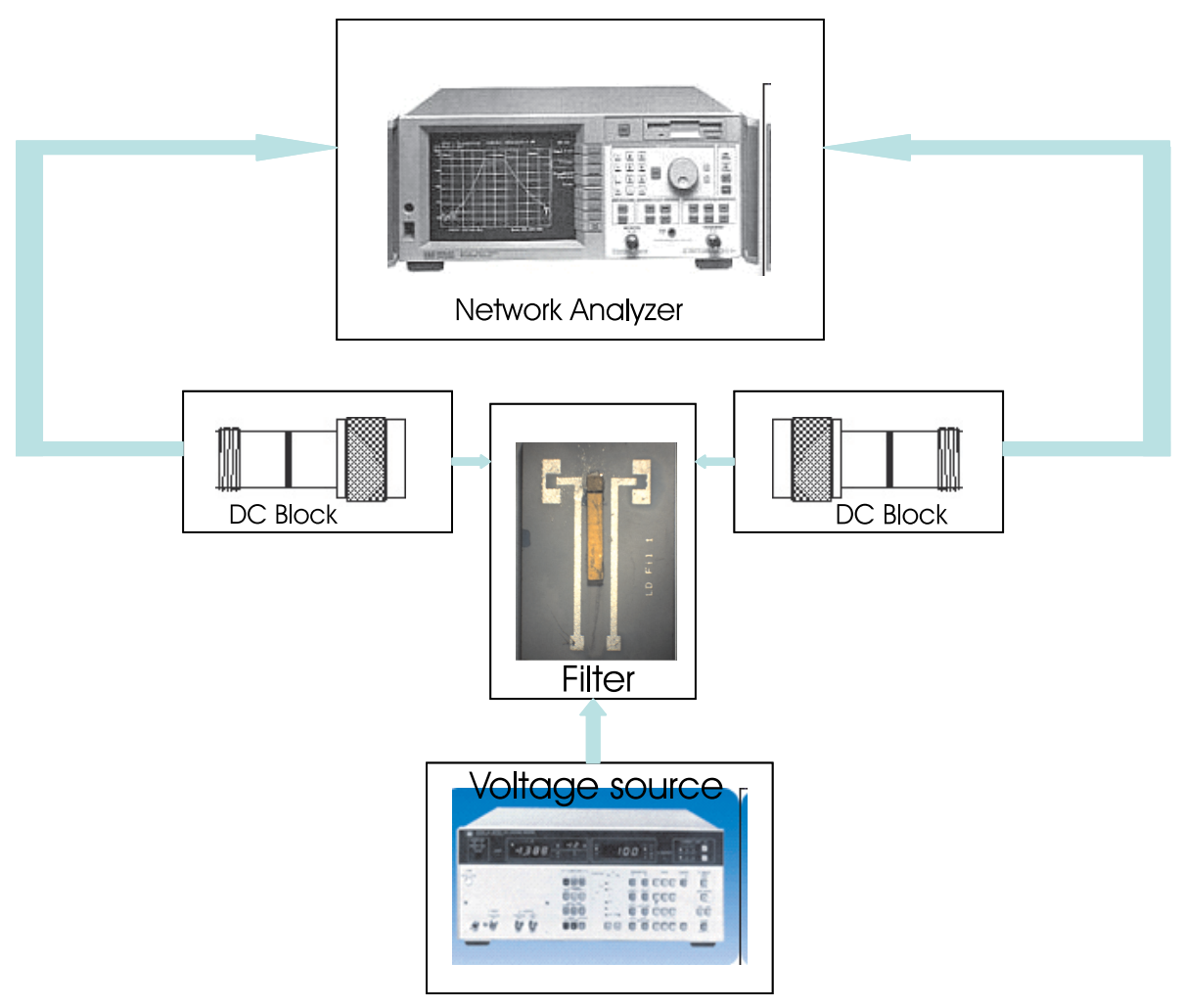

Figure 4.11.: RF measurements setup.

To measure the performance of the fabricated device a DC block capacitors are added at the input and the output port of the Network Analyzer. These capacitors will prevent the DC voltage $(0 \ldots 200 \mathrm{~V})$ from going inside the Network Analyzer and will just allow to the RF signal to pass through. The Network Analyzer that has been used for the measuring of the $\mathrm{S}$ parameters is Agilent 8753.

Where the setup uses external DC Bock capacitors (see Fig. 4.11):

1. Connect the DC Block capacitors to the Network Analyzer.

2. Calibrate the Network Analyzer together with the DC Block capacitors.

3. Connect the RF input and output ports to the filter.

4. Connect the DC voltage source to the cantilever electrodes.

5. Finally, change the applying bias, and measure the S-parameters. 


\subsubsection{Tunability}

The measured response of the filter showed a $135 \%$ tuning range bandwidth from $1.1 \mathrm{GHz}$ to $2.6 \mathrm{GHz}$ with 200 volts control voltage. The filter frequency response was measured under different bias conditions. The measured performance is depicted in Fig. 4.12.

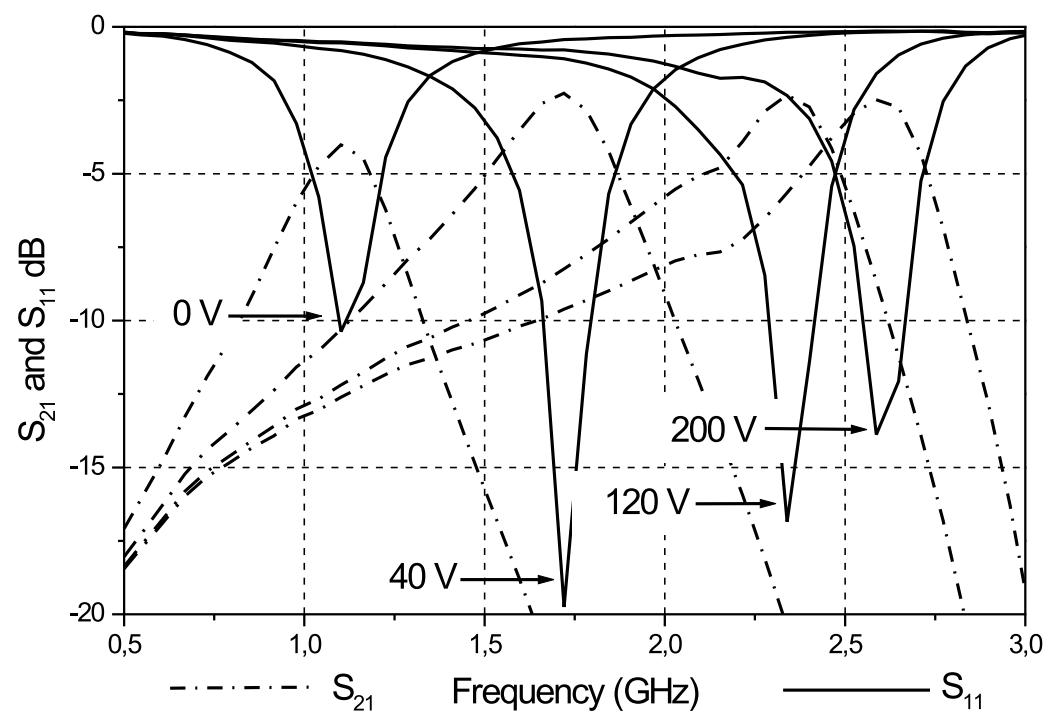

Figure 4.12.: Set of measured insertion loss and return loss responses for various control voltages.

The measured and calculated insertion loss $\left(\left|S_{21}\right|\right)$ of the filter is shown in Fig. 4.13. Simulations are fitted to the measurements by adjusting the effective air gap. The air gap value has been obtained from the sonnet simulation model by changing the thickness of the air layer until the simulation matches the measurements curve. Fig. 4.13 shows also that the absolute bandwidth increases over the tuning range in a manner consistent with constant relative bandwidth, as expected. With a bias voltage up to $200 \mathrm{~V}$ the air gap increases continuously from $2.2 \mu \mathrm{m}$ to $24 \mu \mathrm{m}$. These values were determined by fitting simulated frequency characteristics to measured ones as in Fig. 4.13.

Fig. 4.14 shows the variation of the measured center frequency and of the corresponding air gap as a function of applied DC bias.

\subsubsection{Tunable Filter Parameters}

Fig. 4.15 shows the return loss of the filter as a function of the tuned midband frequency. The filter input matching is good with a return loss of better than $-11 \mathrm{~dB}$ over the tuning range up to $2.6 \mathrm{GHz}$.

The insertion loss reduced over the entire tuning range. The insertion loss is relatively low due to the absence of lossy material. The unloaded quality factor can be found from [86] 


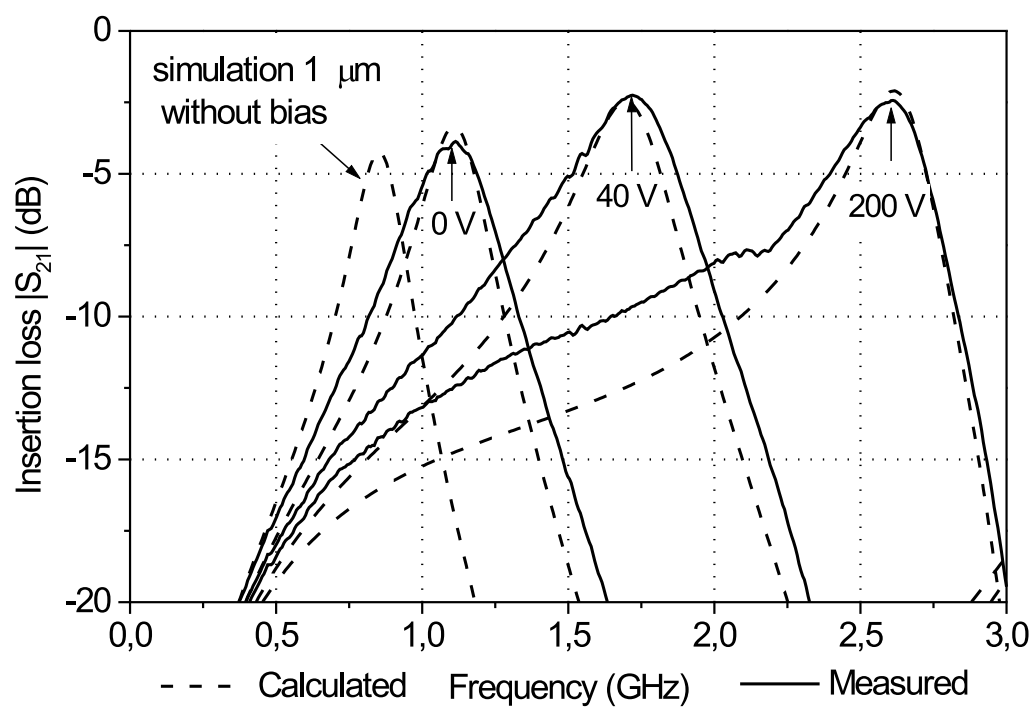

Figure 4.13.: Tuned bandpass filter with variable capacitor.

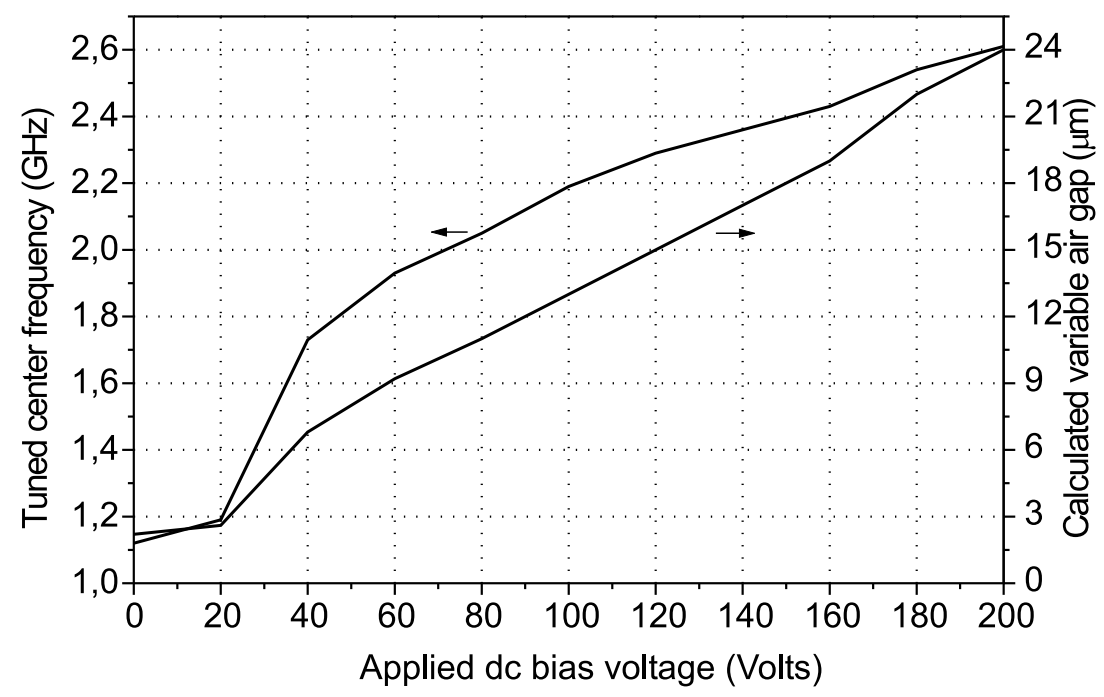

Figure 4.14.: Measured frequency and the calculated corresponding air gap versus applied DC bias. 


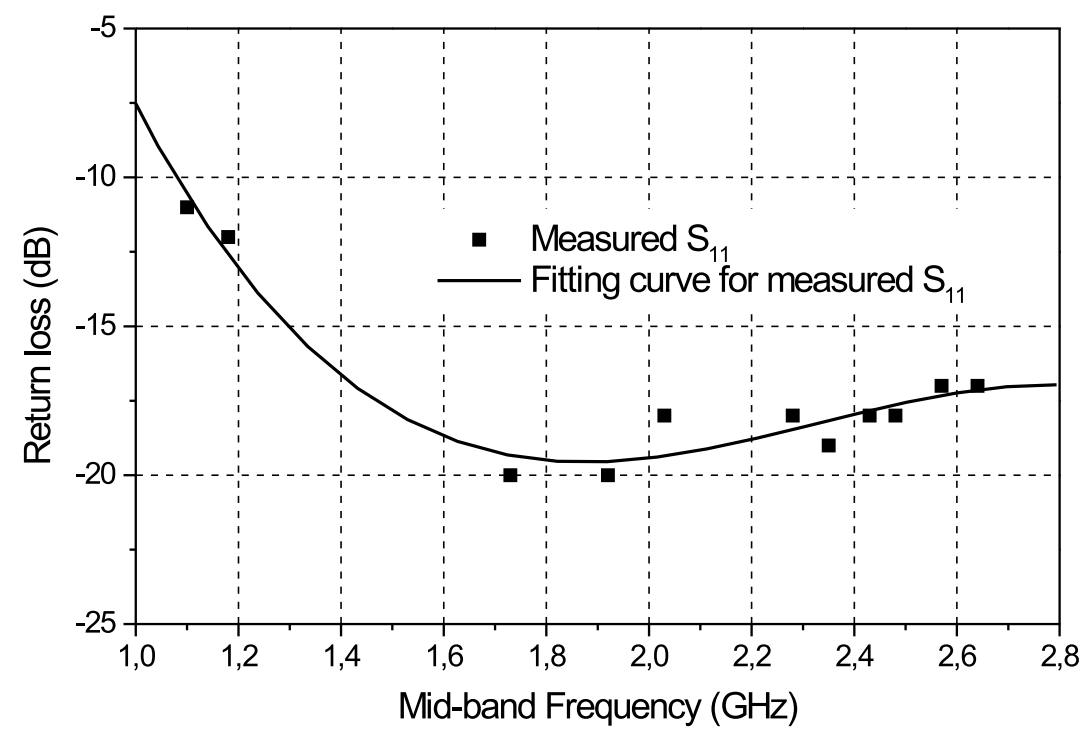

Figure 4.15.: The return loss as a function of the tuned mid-band frequency.

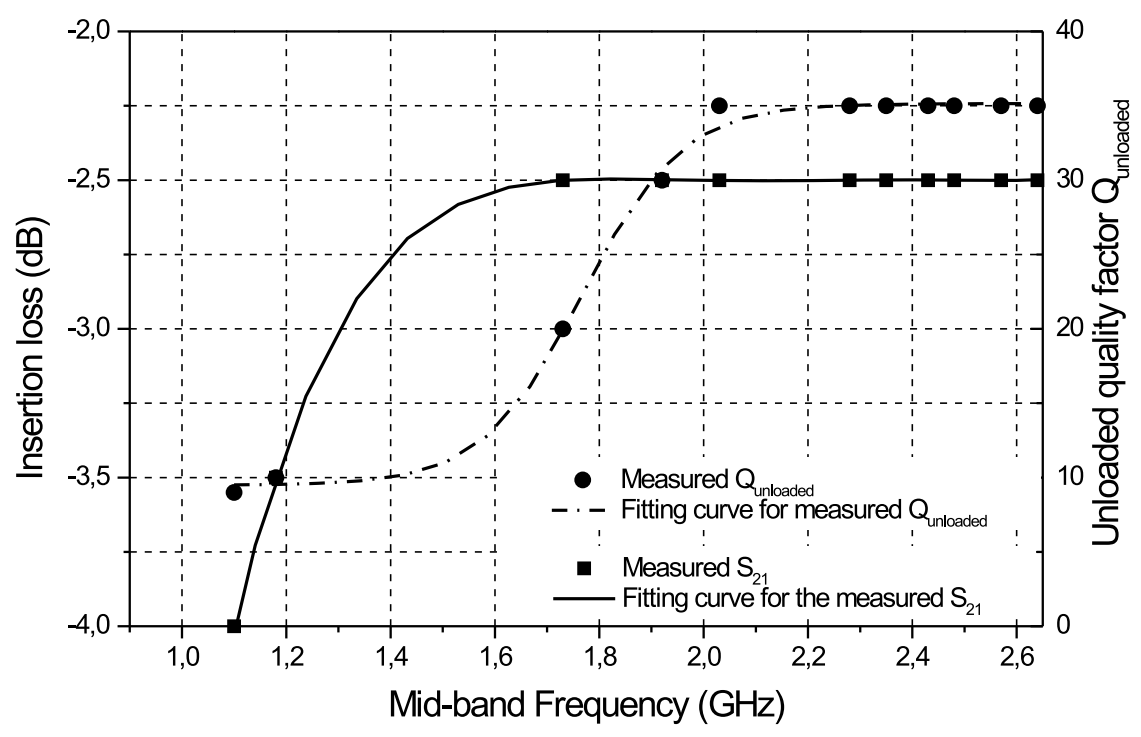

Figure 4.16.: The insertion loss and the unloaded quality factor as a function of the tuned mid-band frequency. 


$$
Q_{\text {unloaded }}=\frac{Q_{\text {loaded }}}{\left(1-S_{21}\left(f_{0}\right)\right)}
$$

where $Q_{\text {loaded }}$ is the loaded quality factor, which can be obtained from measuring the $3 \mathrm{~dB}$ bandwidth of the insertion loss. $S_{21}\left(f_{0}\right)$ is the transmission coefficient of the filter at the midband frequency $f_{0}$. The variation of the midband center frequency and the relative bandwidth are shown in Fig. 4.17

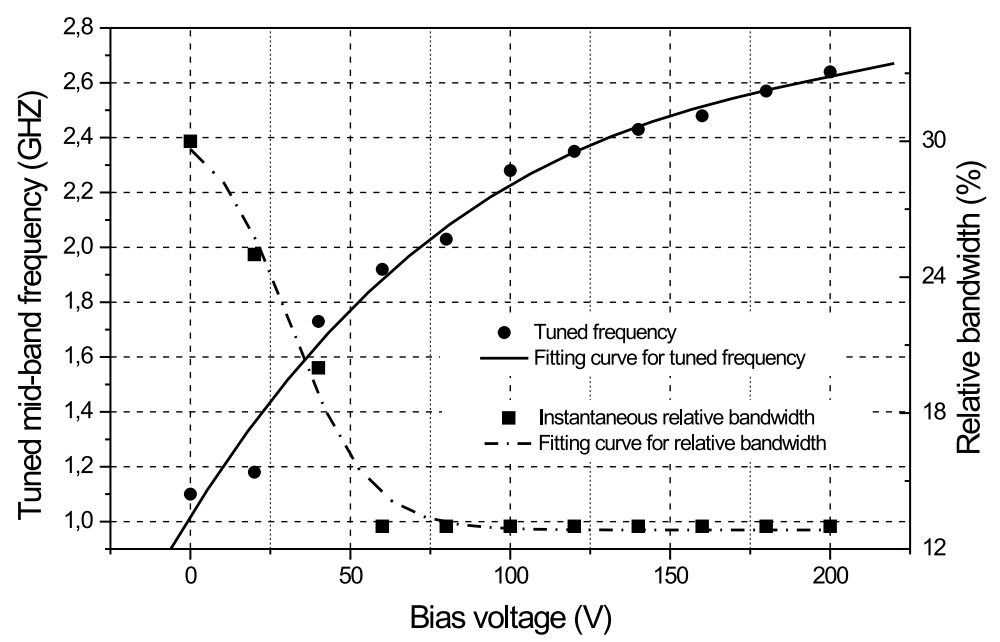

Figure 4.17.: Measured mid-band center frequency and the relative bandwidth as a function of the control bias voltage.

The mid-band frequency is determined by the lengths of the lines which is tuned by the piezoelectric variable capacitor. The bandwidth of the filter depend on the width and the separation of the lines, and not determined by the tuned frequency.

\subsubsection{Lumped Element Representations}

The equivalent circuit of the first-order filter is shown in Fig. 4.18. The filter lines are modeled by the inductors. Spacing between the lines has been modeled by a mutual coupling, $K_{12}, K_{23}$ and $K_{13}$ are the mutual-coupling between lines $0 \& 1,1 \& 2,0 \& 2$, respectively. The ohmic and the dielectric losses of the filter are represented by the resistance $R_{a}$. The capacitor is described by the lower two elements $R_{s}, C$. Table 4.4 shows the equivalent circuit parameters that are not subject to a change during the tuning process. $C, K_{12}, K_{23}$ are the tunable parameters. These parameters have been founded by fitting the equivalents circuit performance to the measurements. The length of the transmission lines have been converted into equivalent lumped inductor elements $L_{1}, L_{2}$, and $L_{3}$. Their values have been computed from the general relation between the distributed and the lumped elements. Since they are of the same length, 


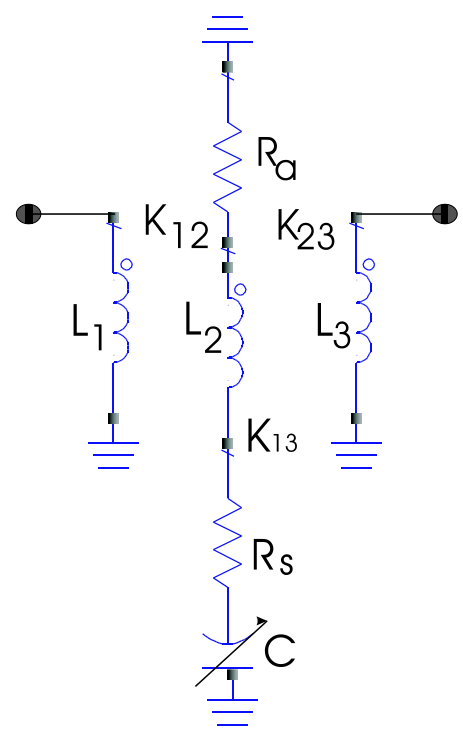

Figure 4.18.: Equivalent circuit of the first order.

their values are identical and are founded to be $3 \mathrm{nH}$ each. Now, the tuned capacitor value has been adjusted by matching the measured $\mathrm{S}$-parameters over the frequency range by varying the effective air gap. The matching was in the tuned center frequency. The coupling between the lines has been computed by adjusting the coupling coefficients $K_{12}$ and $K_{23}$ until the measured and the performance of the equivalent circuit is pretty matched. To account for the dielectric and ohmic losses, numbers of simulations have been run for the case that corresponds to the measured zero-bias voltage. First, the whole setup has been simulated including all the losses of

Table 4.4.: Equivalent circuit fixed parameters of the first order

\begin{tabular}{|l||l|}
\hline$L_{1}=L_{2}=L_{3}$ & $3 \mathrm{nH}$ \\
\hline$K_{13}$ & 0.36 \\
\hline$R_{a}$ for $80 \mathrm{~nm}$ & $0.35 \mathrm{ohm}$ \\
\hline$R_{s}$ for $500 \mathrm{~nm}$ & $0.05 \mathrm{ohm}$ \\
\hline$R_{s}$ or
\end{tabular}

dielectric and metallizations of the structure except for the metallization of the bottom electrode of the cantilever which was lossless. The losses in this case counts for the dielectric losses of the LTCC substrate and the high-K, in addition to the LTCC silver paste metallizations. On the second one, all losses were included, which include the metallization loss of the cantilever. Finally, apart of the metallization of the cantilever that forms the top-movable electrode of area $1 \times 1 \mathrm{~mm}^{2}$ of the capacitor has been simulated as a lossless metal while the rest of the line as a lossy metallization. The all three simulations have been compared with the measured zero-bias case:

- For the first run, the dielectrics losses as well as the LTCC silver paste have been com- 
puted. It is equal to 0.2 .

- For the second run, the losses produced by the cantilever metallization found to be 1.75.

- For the third run, the electrode loss of the capacitor has been computed and is found to be 0.35 .

Thus shows that the metallization of the cantilever is the major contributor to the filter losses. By fitting the model to the measured S-parameters under various biasing voltage the capacitance value changed till the center frequency of the model matched the measured center frequency. The coupling coefficients are varying with the applied voltage. The model shows a broadband fitting across the entire tuning range. The performance of the equivalent circuit of the first order compare to the measurements for at zero-bias is shown in Fig. 4.19.

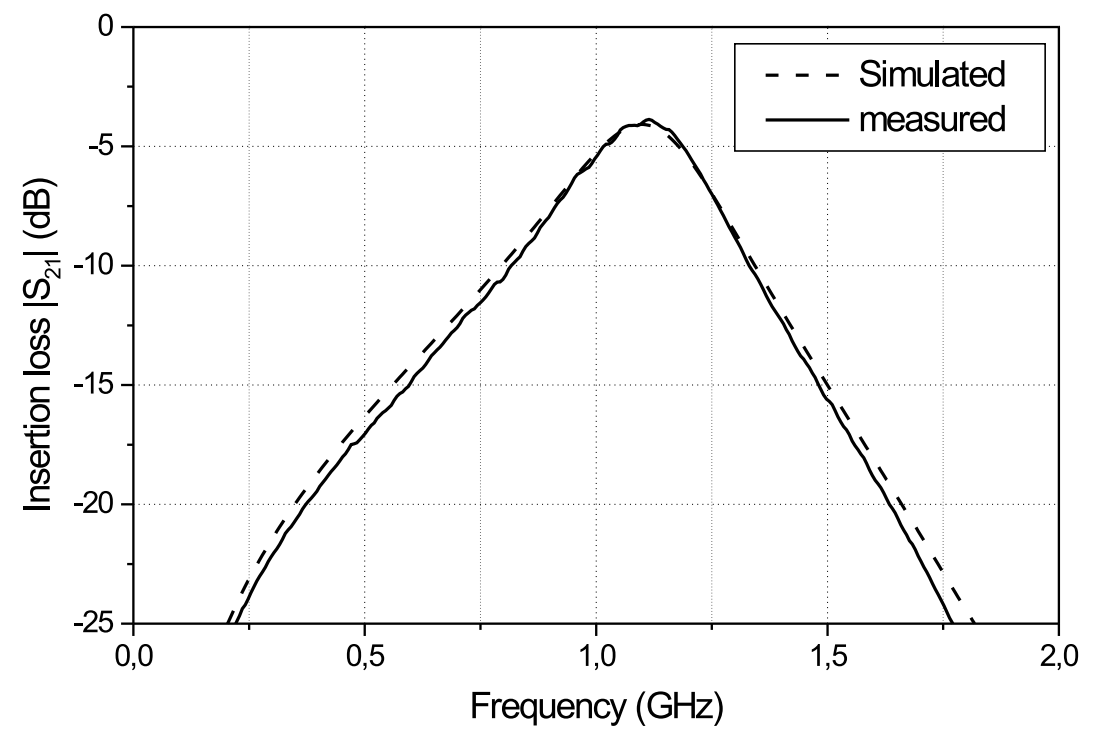

Figure 4.19.: The performance of the equivalent circuit of the first order.

The tuned capacitance and the tuned coupling are shown in Fig. 4.20, and Fig. 4.21, respectively.

\subsubsection{Piezoelectric LTCC Varactor Parameters}

The equivalent circuit of the fabricated filter has been used to explore the change of the capacitor parameters across the entire tuning range. The effective $\epsilon_{r}$ decreases rapidly due to the change in the air gap as shown in Fig. 4.22. The permittivity of the high-K has been dropped from its maximum value 65 at zero air gap to 4.4 at air gap of $2.2 \mu \mathrm{m}$ for zero bias. At the maximum applied voltage a capacitance density of $0.36 \mathrm{pF} / \mathrm{mm}^{2}$ has been measured correspond to an effective air gap of $24 \mu \mathrm{m}$ of $200 \mathrm{~V}$. The maximum capacitance density at zero bias is 3.8 $\mathrm{pF} / \mathrm{mm}^{2}$ resulting in a tuning ration of $11: 1$ (Fig. 4.23).

Effective air gap has been founded by using the simulation model that has been developed in 


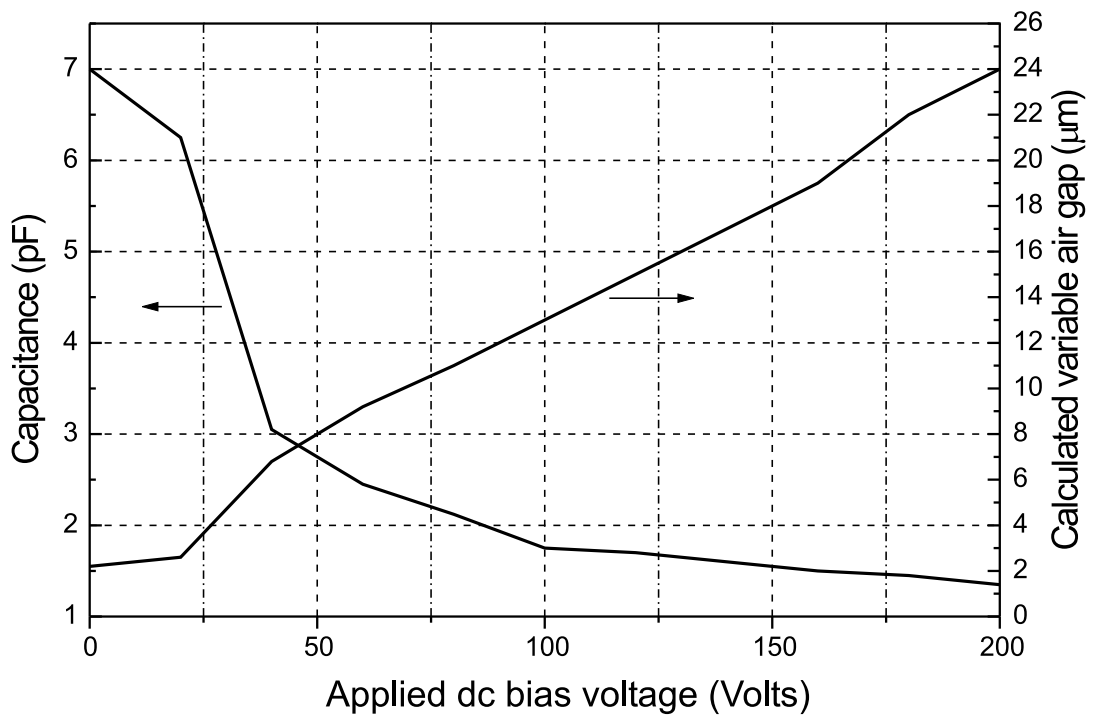

Figure 4.20.: Measured capacitance and the calculated corresponding air gap versus applied DC bias.

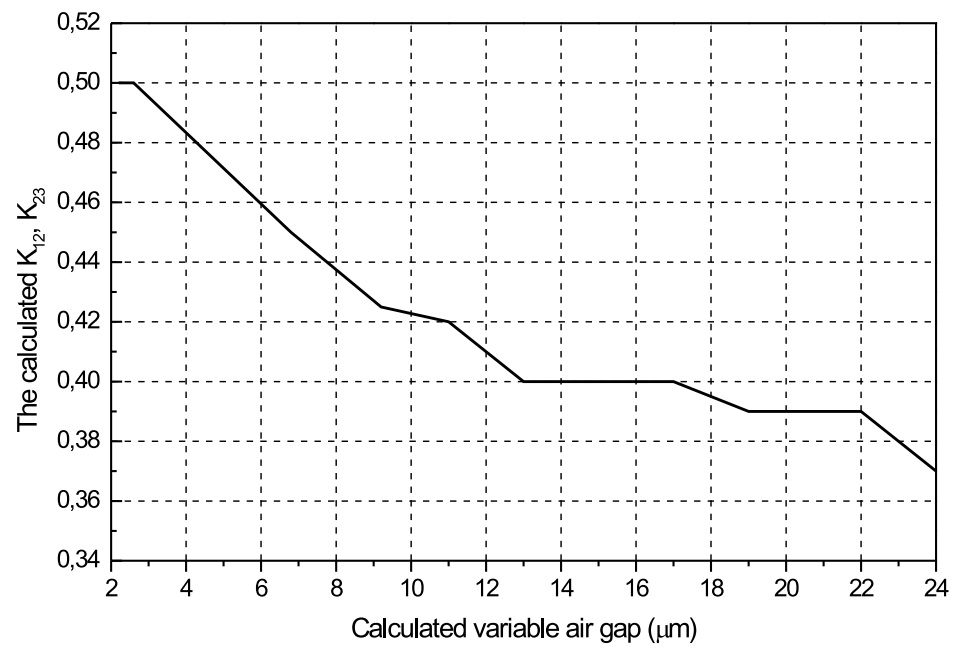

Figure 4.21.: Measured tunable coupling as a function of the deflection. 


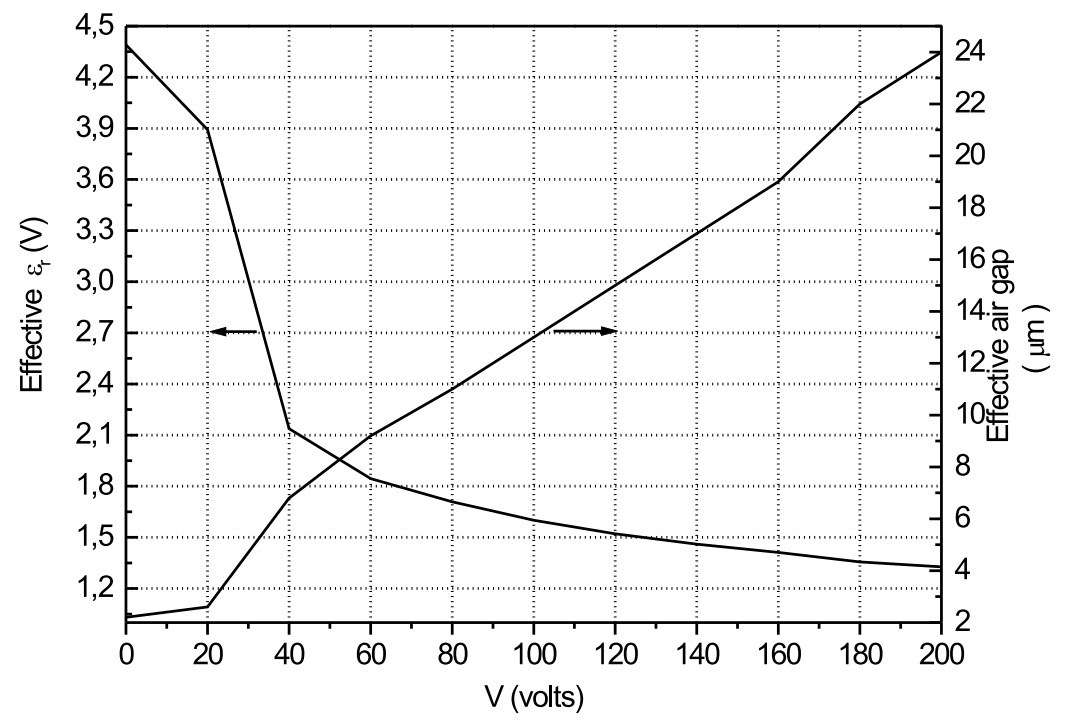

Figure 4.22.: Effective permittivity and the corresponding air gap versus actuation voltage.

section xx. From Fig. 4.23 it can be seen that as the air gap increases inside the capacitor the frequency goes up. This relationship is not linear. It also could be seen that when the air gap is small, the amount of frequency variation is large compare to the big air gap. The real air gap depends mainly on the assembly procedure which might reduce the effective free-length of the cantilever.

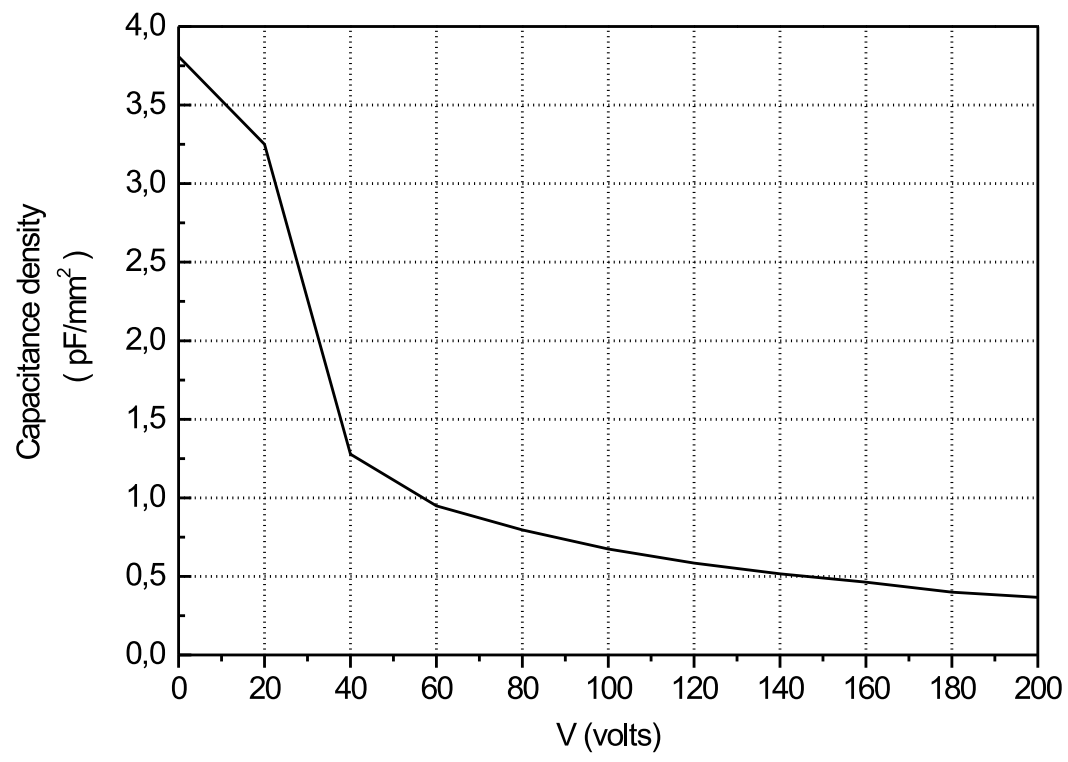

Figure 4.23.: Capacitance density versus actuation voltage.

The quality factor of the capacitor at mid band frequency has been determined from the equivalent circuit and the relation [80]: 


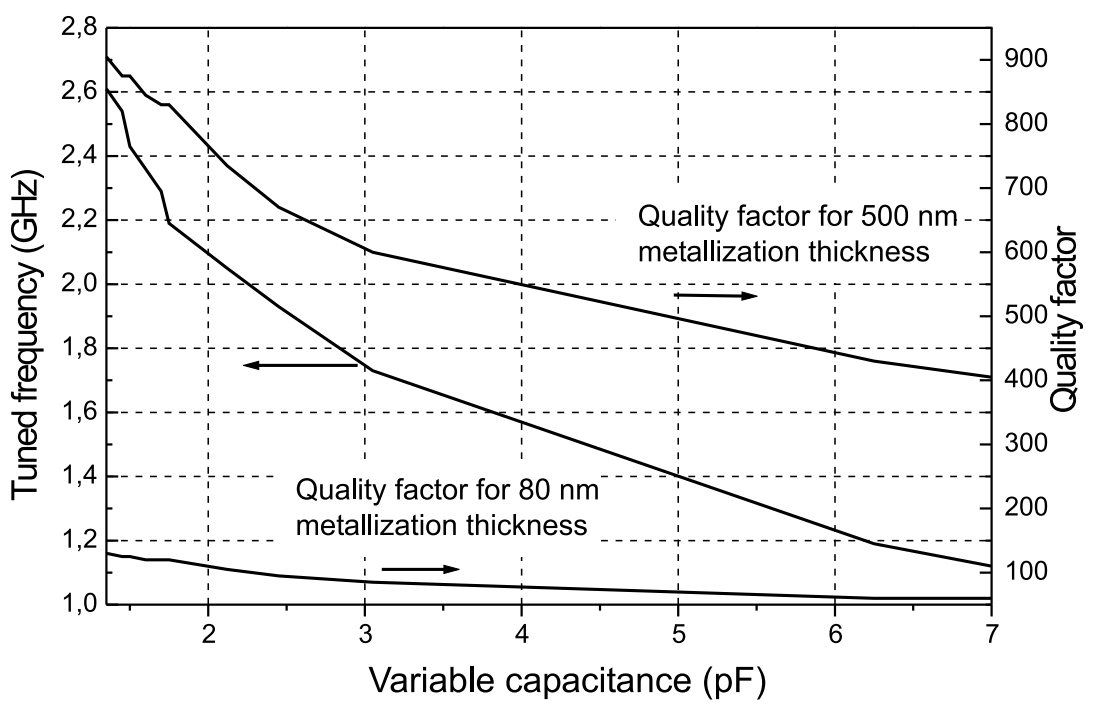

Figure 4.24.: Measured tunable frequency and the quality factor versus capacitance variation.

$$
Q=\frac{1}{2 \pi R_{s} f C}
$$

The DC resistance of the cantilever electrode with $80 \mathrm{~nm}$ gold is found to be $2.2 \mathrm{ohm}$, and 0.35 ohm for $500 \mathrm{~nm}$ gold. Fig. 4.24 shows the measured tuned filter center frequency, the measured quality factor at $80 \mathrm{~nm}$ gold, and the calculated quality factor for $500 \mathrm{~nm}$ gold.

\subsection{Higher Order Filters}

As with any other filter technology, quality factor and selectivity can be improved at the expense of insertion loss, e.g. by adding further circuit elements or resonators. The higher the order is, the sharper the attenuation of the filter. A two-poles "second-order" combline filter has been designed and fabricated. This chapter treats the measurements and characterizations of this filter. The filter has shown a better selectivity of 10 to $15 \mathrm{~dB}$ than the first order. The quality factor of the capacitor has been improved by a factor of 2 for a cantilever metallization of 150 nm gold thickness.

\subsubsection{Filter layout}

The layout of the two-poles filter consists of four coupled microstrip lines is shown in Fig. 4.25. The increasing in the order has been done to improve the selectivity, the quality factor, and the attenuation. As in the first order filter, the two interior lines have been divided into two sections each. One of the two sections of the interior lines (RF1A/RF1B) is identical to the bottom metallization of the piezoelectric cantilever (A \& B, respectively), which each is shortened to 


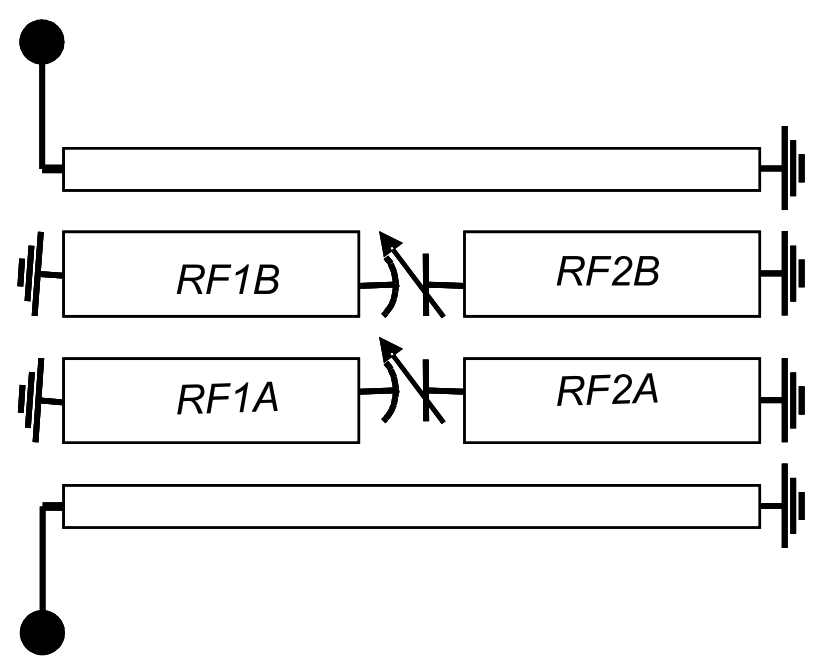

Figure 4.25.: Layout of the 2-pole tunable filter.

ground at one end, and on the other end it forms the upper-movable electrodes of the two variable capacitors. The second sections of the lines (RF2A/RF2B) are comprised on the top of the LTCC substrate, thus each forming the bottom-fixed electrodes of the capacitors. The filter has been designed using the developed theory of chapter four. Table 4.5 summarize all filter dimensions.

Table 4.5.: Filter physical dimensions of the second order

\begin{tabular}{|l||l|}
\hline Widths of lines the input \& output $(\mathrm{w})$ & $0.5 \mathrm{~mm}$ \\
\hline Width of the center coupled line $\left(W_{m}\right)$ & $1.0 \mathrm{~mm}$ \\
\hline Spacing between the lines $(\mathrm{S})$ & $0.25 \mathrm{~mm}$ \\
\hline Lengths of lines $(\mathrm{l})$ & $11.4 \mathrm{~mm}$ \\
\hline Spacing between the cantilevers $\left(S_{m}\right)$ & $1 \mathrm{~mm}$ \\
\hline Filter size & $12 \times 9 \mathrm{~mm}^{2}$ \\
\hline
\end{tabular}

\subsubsection{The Fabrication of the Filter}

The filter has been fabricated using the same fabrication process (see 4.6: "Device-Specific Fabrication Process") as in the first order. The simplified fabrication process is shown in Fig. 4.26. The metallization of the bottom side of the cantilevers have a $150 \mathrm{~nm}$ gold thickness.

1. The fixed two sections of the interior lines (RF2A \& RF2B), which forms the lower electrodes of the tunable capacitors at one of their ends are printed on top of the $1.2 \mathrm{~mm}$ thick substrate (Fig. 4.26a). 
(a)

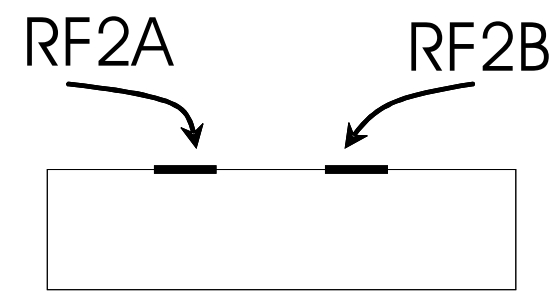

(b)

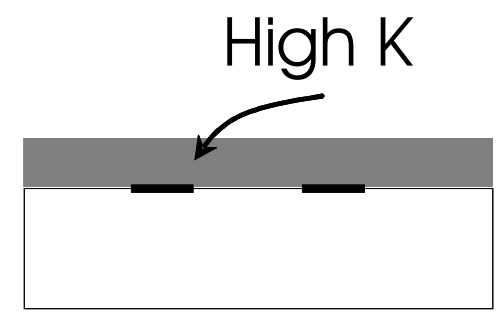

(c)

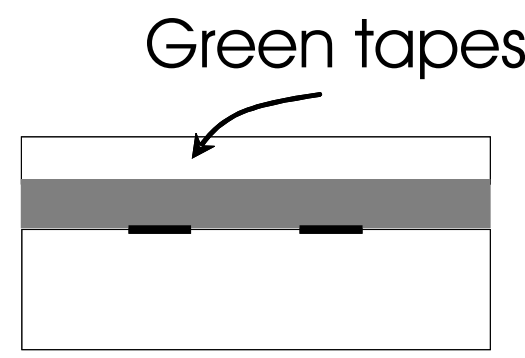

Polishing

(d)

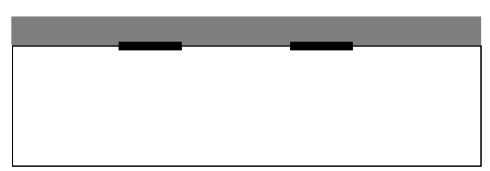

Line 1

(e)
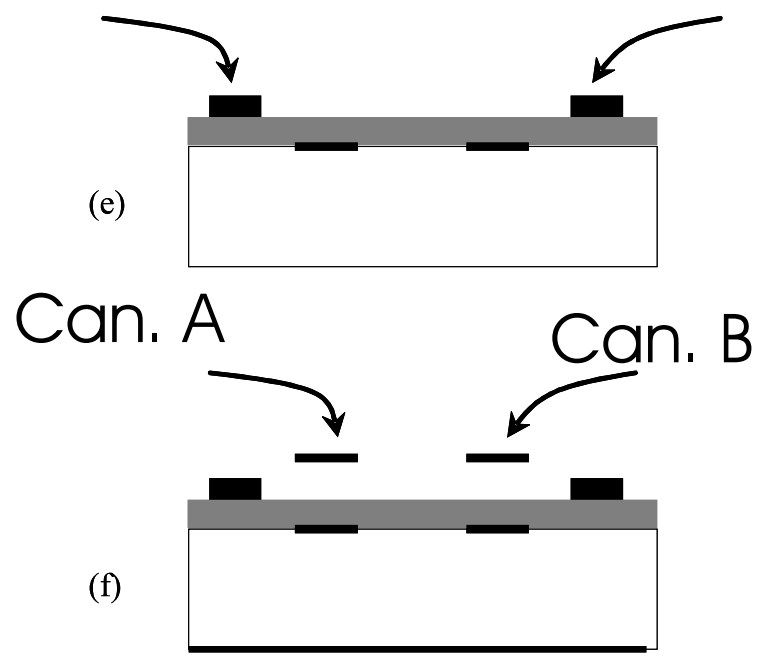

Figure 4.26.: Simplified fabrication process for the second order.

2. As in the first order fabrication process a proprietary high-K tape is used to provide the highly dielectric layer between the capacitors electrodes (Fig. 4.26b). To balance shrinkage and thermal stress during sintering, a symmetric stack of tapes is added on top, which also provides sacrificial material for the polishing step (Fig. 4.26c).

3. The whole layers are then laminated and then co-fired at around $850^{\circ} \mathrm{C}$.

4. After the sintering process, the bottom surface is slightly polished for the planarity of the top surface. The sacrificial material at the top is removed completely, and the polishing process is proceeded until the desired thickness of the high-K tape $(10 \mu \mathrm{m}$, permittivity 65, loss tangent 0.002) and a smooth flat surface is reached (Fig. 4.26d).

5. The microstrip coupled lines $1 \& 2(\mathrm{w}=0.5 \mathrm{~mm}, \mathrm{~s}=0.25 \mathrm{~mm}, \mathrm{~L}=11 \mathrm{~mm})$ and the ground metallization are screen printed on both top and bottom surfaces (Fig. 4.26e).

6. The movable part of the interior lines (RF1A \& RF2B) are identical with the cantilever bottom metal $(\mathrm{w}=1 \mathrm{~mm}, \mathrm{~L}=7 \mathrm{~mm}$ ). They overlap with the thick-film section (RF2A \& RF2B, respectively) on the high $-\mathrm{K}$ material to form a capacitors area of $1 \times 1 \mathrm{~mm}^{2}$ (Fig. 4.26f).

Finally, the same assembly procedure of the first order has been used for the second order. The cantilevers fixed one after one. 


\section{The Fabricated device}

Fig. 4.27 shows a photograph of the final device. Above the high-K tape, the input and the output lines $(1 \& 2)$ ended with via pads from one-end and on the other-end form the input/output GSG pads. The design uses $800 \mu \mathrm{m}$ GSG pads, the same in the first order. The bottom electrode of the used piezoelectric actuators have been used as one of interior coupled line sections (RF1A \& RF1B). It is shown that one-end of the cantilevers is fixed to the LTCC substrate by glue, and the other end left free. The other sections of the interior coupled lines (RF2A \& RF2B) are embedded in between the high-K tape and the LTCC substrate, they are invisible. The variable capacitors are formed by the overlapping area between the sections of the interior lines each. The angular misalignments between the fixed lines (1\& 2) and the movable lines (RF1A \& RF1B) could be noticed. The overall size is approximately $12 \times 9 \mathrm{~mm}^{2}$.

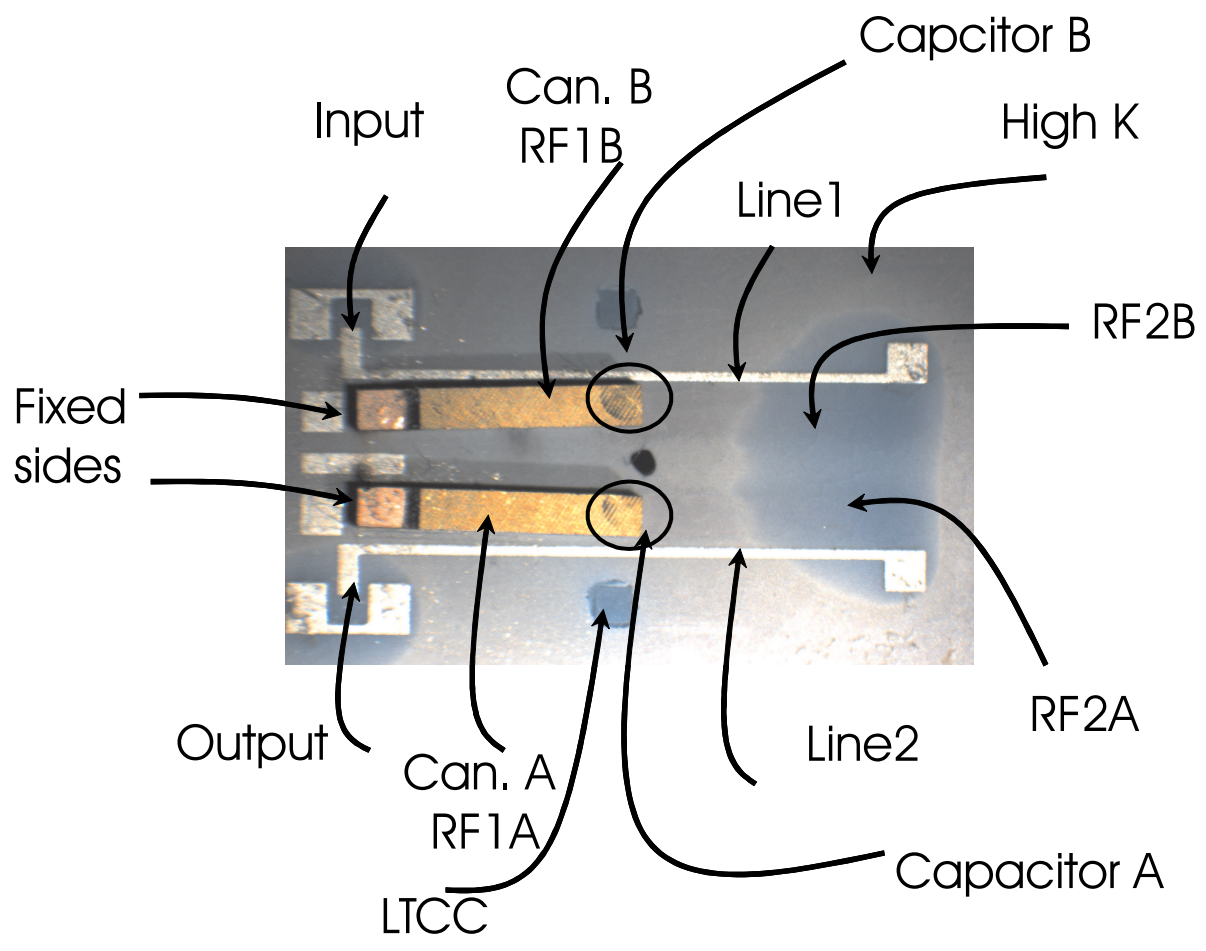

Figure 4.27.: The fabricated second order filter.

\subsubsection{RF Measurements}

The two cantilevers (A \& B) have been driven individually and simultaneously in order to make the two capacitances values identical to each other. The measurements of the performance of the filter are shown in Fig. 4.28. It has been possible to adjust the values of the two capacitors (A \& B ) identical to each other in the frequency window of width $0.57 \mathrm{GHz}$, between 1.23 $\mathrm{GHz}$ and $1.8 \mathrm{GHz}$. The filter shows a $46 \%$ tuning range. The filter has been simulated and compared successfully to the measurements as shown in Fig. 4.29. The simulations have been 


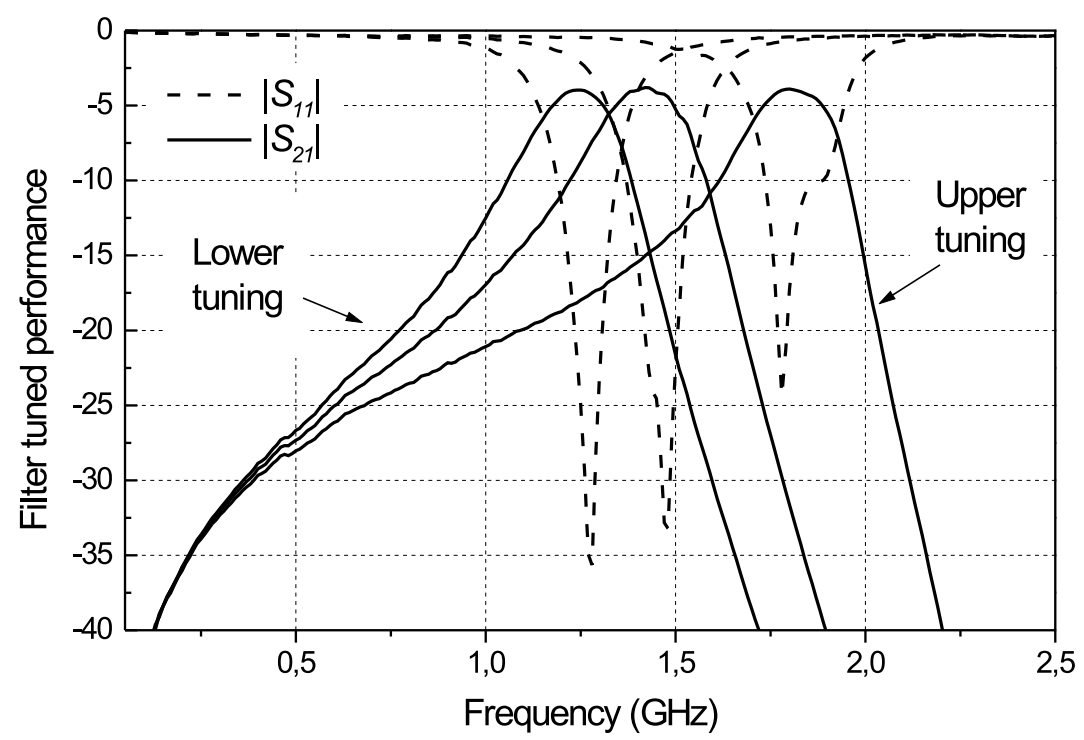

Figure 4.28.: Measured tuning curves for the second order filter.

carried by using sonnet tools by adjusting the effective air gap. The deviations is due to the angular misalignments of the two cantilevers in both the vertical and the horizontal planes.

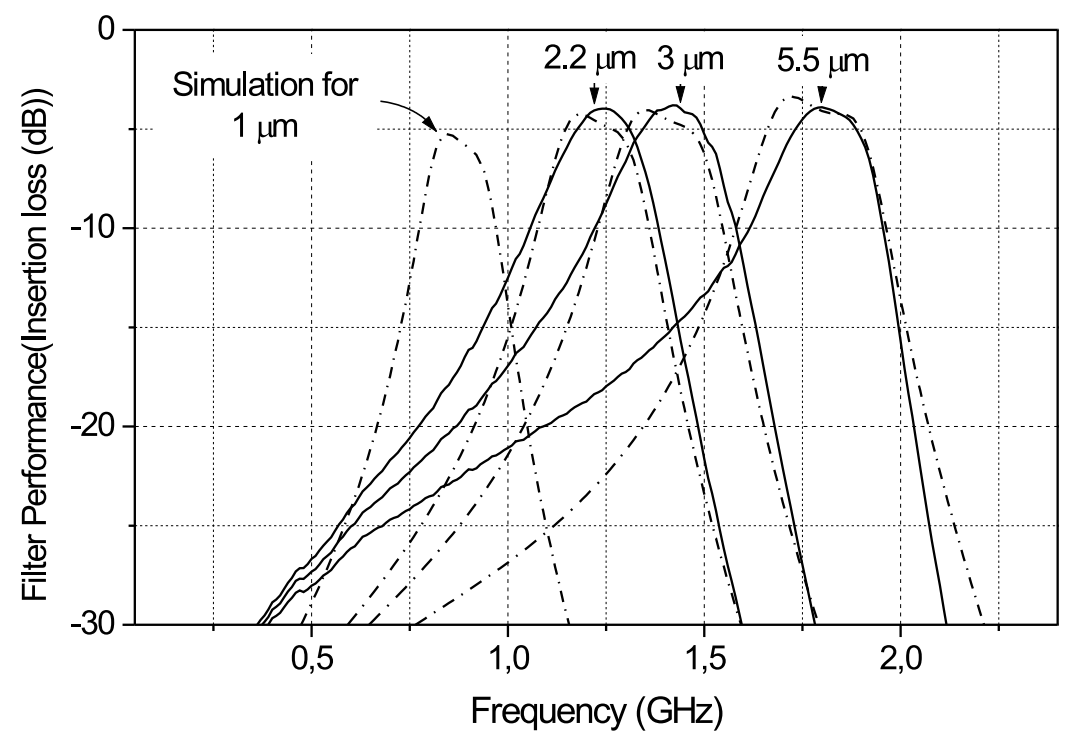

Figure 4.29.: Measurements and simulations of the second order.

\section{Tunability in the Second-Order}

After the connection of the voltage sources to the electrodes of the two cantilevers, the filter performance for zero bias is shown in Fig. 4.30. The bias on the cantilever B has been increased to shift the return loss $S_{22}$ down to match $S_{11}$, the matching case is the lower tuning curve of 


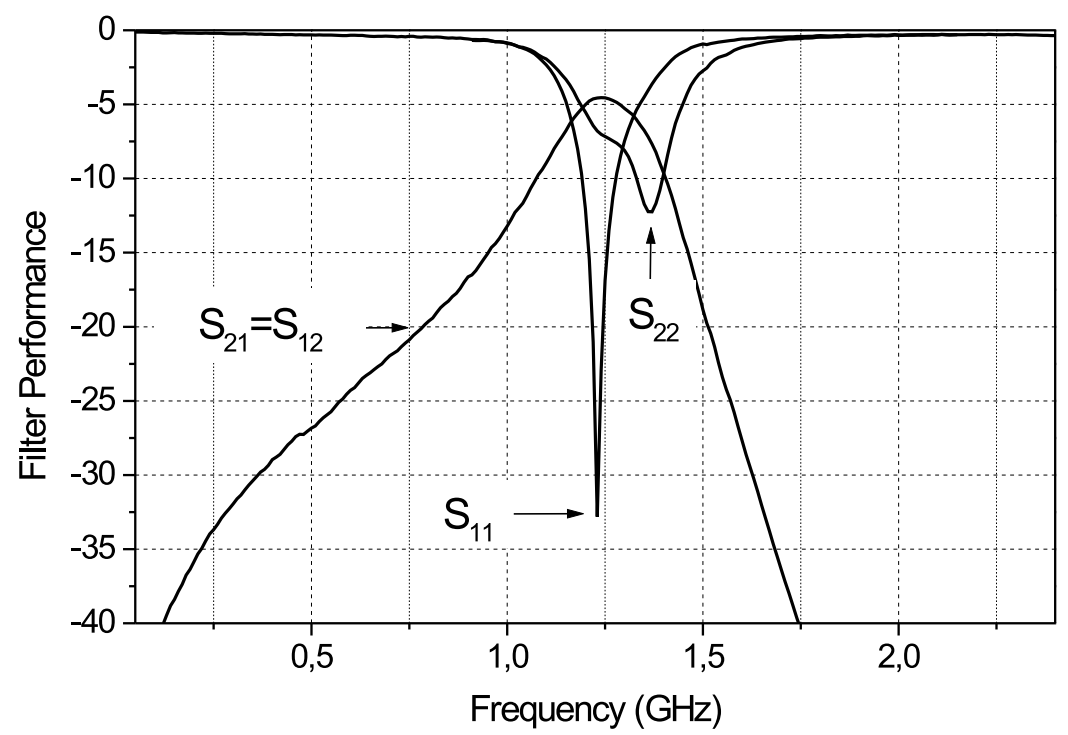

Figure 4.30:: The most lower tuning range could be achieved within this sample.

Fig. 4.28. At this case, cantilever B has been blocked to move further down because of the roughnesses of the LTCC surface, so the tuning has been blocked in the lower-range. The two cantilevers have been adjusted to reach the upper-tuning curve of Fig. 4.28. Then the cantilever A has been blocked by the assembly process, thus the upper-tuning range has been blocked, while cantilever B still going further up, the result of the maximum deflection is show in Fig. 4.31.

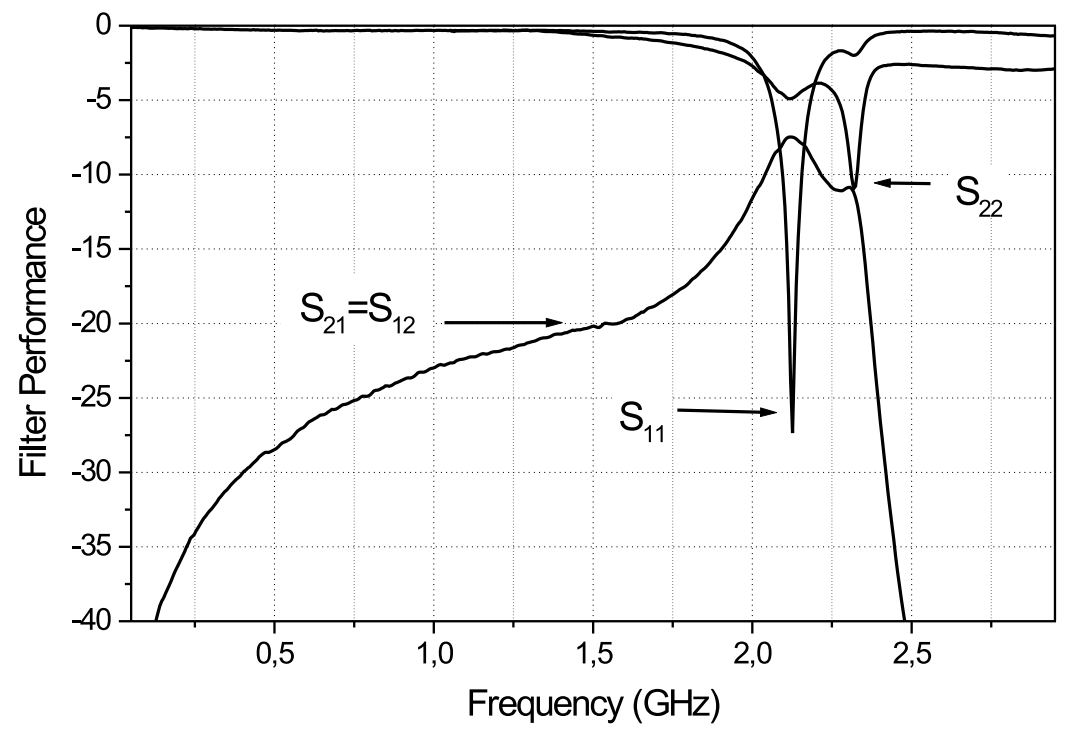

Figure 4.31.: The most upper tuning range could be achieved within this sample.

The device performance under different bias voltages has been measured. At first, the voltage has been applied to cantilever A from 0 to $200 \mathrm{~V}$, while B is kept at zero bias. The measured 
tuned frequency for the input $\left(S_{11}\right)$ has been taken and shown on the left axis of Fig. 4.32. The

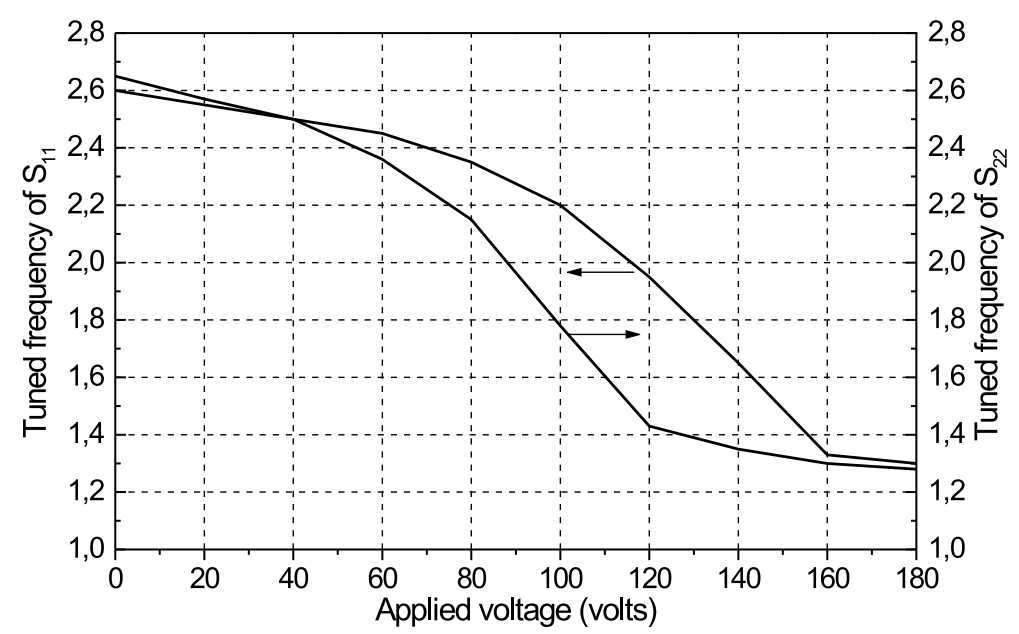

Figure 4.32.: The tuned frequency for the input $\left(S_{11}\right)$ and the output $\left(S_{22}\right)$ reflection loss versus the actuation voltage.

zero-voltage has been fixed to zero on cantilever A, and changed from 0 to $200 \mathrm{~V}$ at cantilever B. The tuned frequency of the output $\left(S_{22}\right)$ reflection loss has been taken and is shown at the left curve and right axis of Fig. 4.32 .

\subsubsection{Attenuation}

The attenuation of the filter has been improved by 10 to $15 \mathrm{~dB}$. Fig. 4.33 compared the insertion loss for both the first and the second order. A better selectivity has been achieved. From the measured filter, the performance of frequency skirt enhances the isolation.

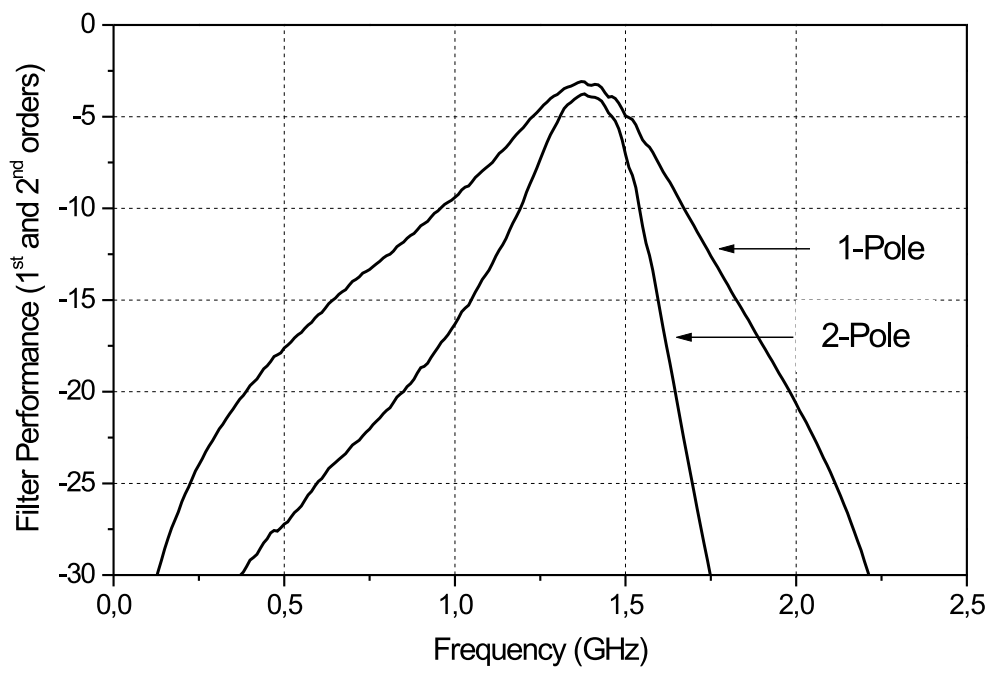

Figure 4.33.: Comparison: measured attenuation for the first and the second orders. 


\subsubsection{Equivalent circuit}

The equivalent circuit of the filter is shown in Fig. 4.34. The input and the output lines has been modelled by the inductors $L_{1}, L_{4}$, respectively. The two interior lines have been modelled by the inductors $L_{2} \& L_{3}$. The two capacitors which formed by the overlapping areas of the cantilevers $\mathrm{A} \& \mathrm{~B}$ have been modelled by $C_{1}, C_{2}$, respectively. The mutual couplings between the line which modelled as $L_{1}$ and the other lines $\left(L_{2}, L_{3} \& L_{4}\right)$ are modelled by $K_{12}, K_{13}, K_{14}$, respectively. $K_{23}, K_{24}$, the mutual couplings between lines $L_{2}, L_{3}$, respectively. $K_{34}$, the mutual coupling between lines $L_{3} \& L_{4}$. The ohmic and the dielectric losses of the filter are represented

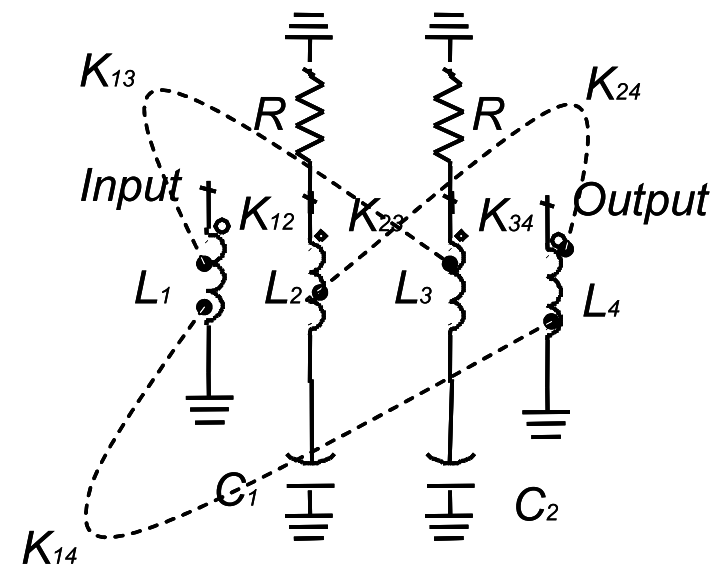

Figure 4.34.: Equivalent circuit of the second order filter.

by the resistance $R$. Table I shows the equivalent circuit parameters that are not subject to a change during the tuning process. $C, K_{12}, K_{23}$ are the tunable parameters that we are going to explore.

Table 4.6.: Equivalent circuit fixed parameters of the second order filter

\begin{tabular}{|l||l|}
\hline$L_{1}=L_{2}=L_{3}=L_{4}$ & $3 \mathrm{nH}$ \\
\hline$K_{14}$ & 0.08 \\
\hline$R$ & $1 \mathrm{ohm}$ \\
\hline$K_{24}=K_{13}$ & 0.1 \\
\hline$K_{23}$ & 0.11 \\
\hline
\end{tabular}

As in the first order, the length of the transmission lines have been converted into their corresponding lumped inductor elements $L_{1}, L_{2}, L_{3}$, and $L_{4}$. Their values have been computed from the general relation between the distributed and the lumped elements. Since they are of the same length, their values are identical and are founded to be $3 \mathrm{nH}$ each. Now, the tuned capacitors values have been adjusted by matching the measured S-parameters over the frequency range by varying the effective air gap. The matching was in the tuned center frequency. 
The coupling between the lines has been computed by adjusting the coupling coefficients $K_{14}$, $K_{13}, K_{24}$, and $K_{23}$ until the measured and the performance of the equivalent circuit is pretty matched. To account for the dielectric and ohmic losses, numbers of simulations have been run for the case that corresponds to the measured zero-bias voltage. First, the whole setup has been simulated including all the losses of dielectric and metallizations of the structure except for the metallization of the bottom electrode of the cantilever which was lossless. The losses in this case counts for the dielectric losses of the LTCC substrate and the high-K, in addition to the LTCC silver paste metallizations. On the second one, all losses were included, which include the metallization loss of the cantilever. Finally, apart of the metallization of the cantilever that forms the top-movable electrode of area $1 \times 1 \mathrm{~mm}^{2}$ of the capacitor has been simulated as a lossless metal while the rest of the line as a lossy metallization. The all three simulations have been compared with the measured zero-bias case:

- For the first run, the dielectrics losses as well as the LTCC silver paste have been computed. It is equal to 0.2 .

- For the second run, the losses produced by the cantilever metallization found to be 0.68.

- For the third run, the electrode loss of the capacitor has been computed and is found to be 0.11 .

The performance of the equivalent circuit is superimposed with the measurements in Fig. 4.35.

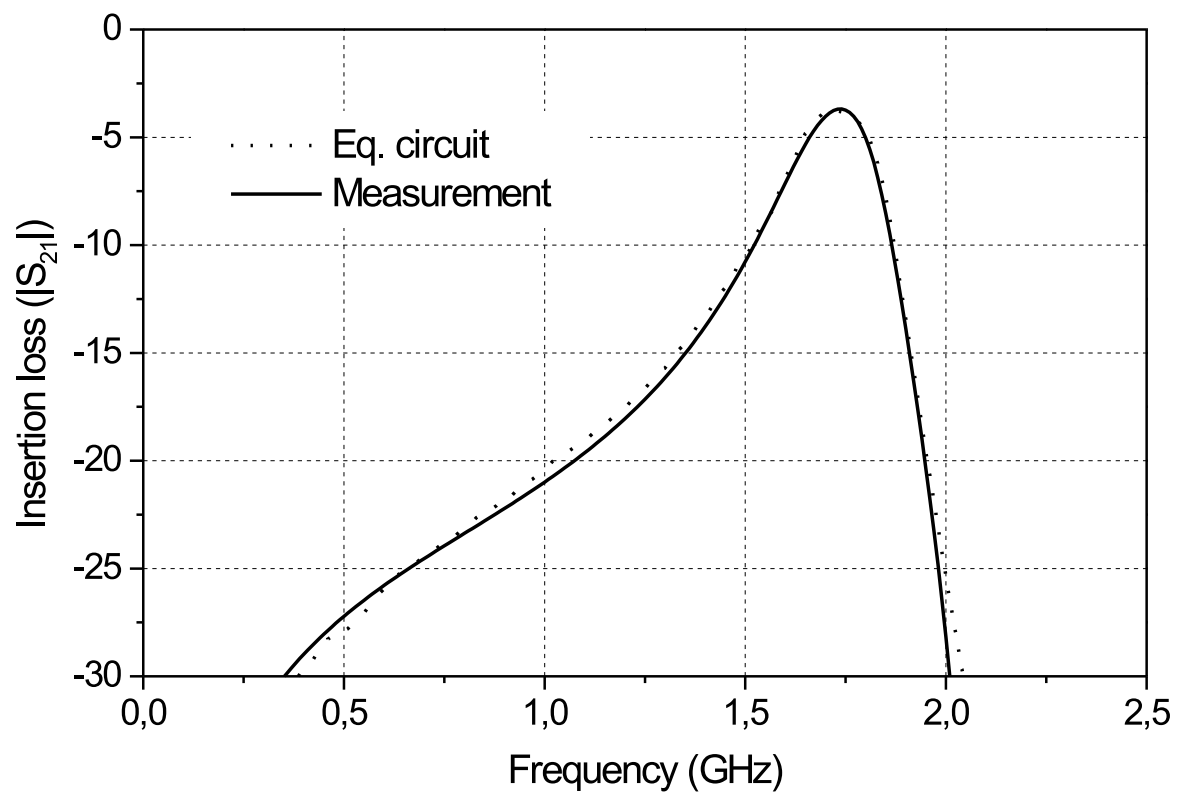

Figure 4.35.: Equivalent circuit and measurements.

Thus shows that the metallization of the cantilever is the major contributor to the filter losses. By fitting the model to the measured S-parameters under various biasing voltage the capacitance value changed till the center frequency of the model matched the measured center 
frequency. The coupling coefficients are varying with the applied voltage. The model shows a broadband fitting across the entire tuning range.

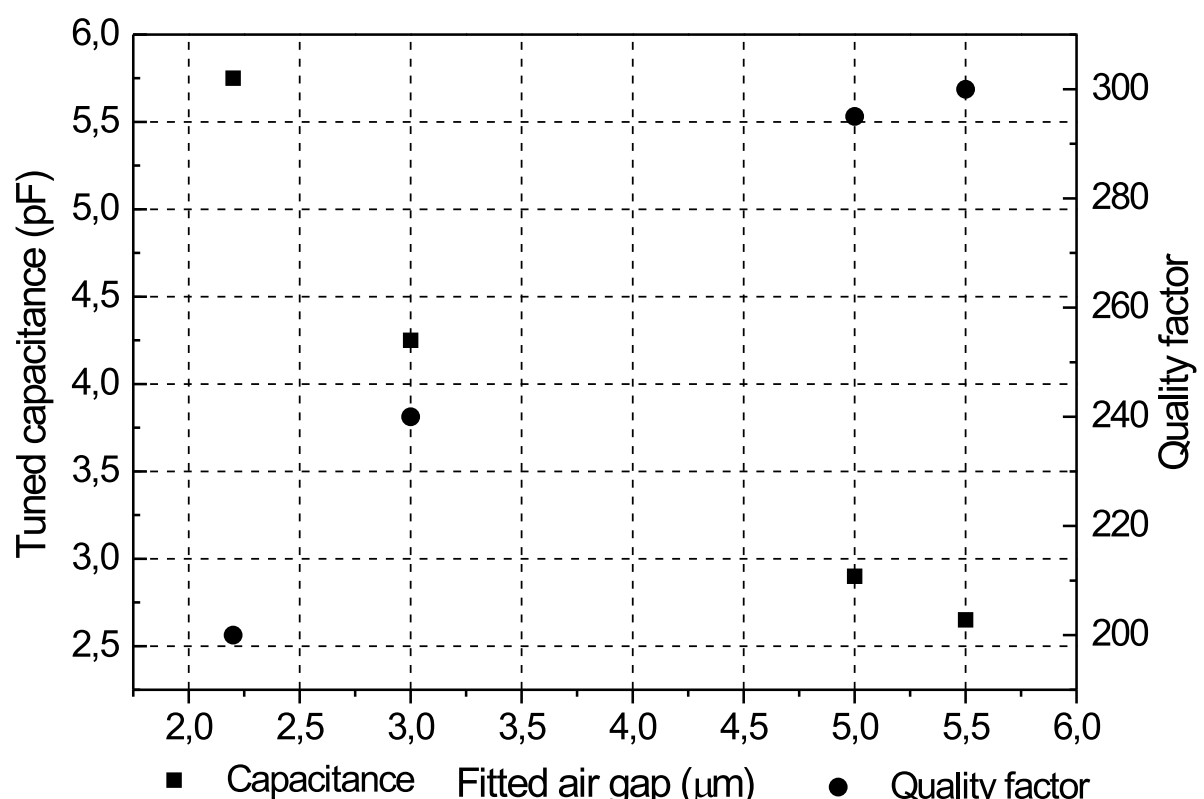

Figure 4.36.: Tuned capacitance and quality factor versus variable air gap of the second order filter.

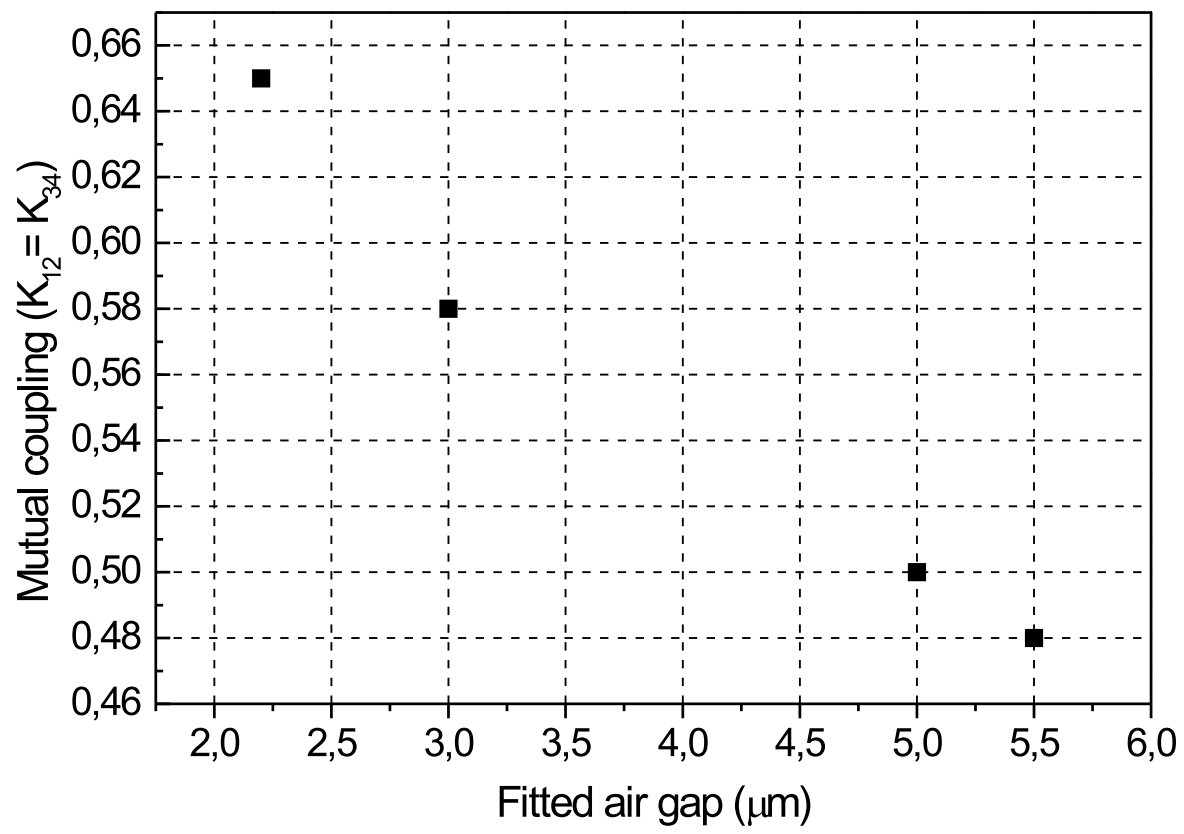

Figure 4.37.: Tuned mutual coupling versus variable air gap of the second order filter.

The tuned capacitance and the corresponding quality factor are shown in Fig. 4.36. The capacitance has been tuned from $5.75 \mathrm{pF}$ to $2.65 \mathrm{pF}$ with a high quality factor between 200 
and 300, in the tuning window. The tuned mutual couplings, $K_{12}$ and $K_{34}$ are shown in Fig. 4.37. 


\section{Interpretation}

The measurement results are analyzed and interpreted in this chapter. The device shows a word record tuning range and a relative low insertion loss with respect to the tunable filters were published. The analysis of the device by full-wave simulation reveals a potential tuning range from $0.8 \mathrm{GHz}$ to $2.8 \mathrm{GHz}$ when the thin-film processability of the LTCC surface is properly controlled. Switching speed, dynamic behaviors as well as power consumption are being addressed in this chapter.

\subsection{Performance Analysis}

In this section the measured device performance is going to be analyzed and to be compared with the state of the art. Since the complete filter is exhibits tunable capacitances, filter performance, such as return loss, will degrade when the passband frequency is tuned away from the center passband frequency for which the filter has been designed. Moreover, this design depends on the electromagnetic coupling between resonators to provide the required impedance inverting elements. Such coupling is frequency dependent and thus the designed impedance inverters only operate correctly over narrow bandwidths. Hence, tuning over a broad range produces deterioration in passband return loss.

\section{mechanical movement}

This is a novel tunable bandpass filter joining for the first time on the level of functional devices two ceramic technologies, LTCC piezoelectric actuator technology. In lieu of Hrovat et al. [71]: the LTCC and the PZT components has been cofired separately and then glued together. A mechanical movement occurred for the first time in this type of devices. The used cantilever has the ability to bend both upwards and downwards by proper biasing. In one of the fabricated samples, it shows a bending of $8 \mu \mathrm{m}$ towards the LTCC substrate, and maximum upward deflection of $32 \mu \mathrm{m}$. In the downward the cantilever is blocked on this sample by the substrate. The finite extension of the glue also limits the maximum upward deflection far away from the LTCC substrate, in the free space.

\section{tunable frequency range}

The center frequency of the filter has be tuned from $1.1 \mathrm{GHz}$ (zero-bias) to $2.6 \mathrm{GHz}$ (200 V-bias) resulting in $(135 \%)$ tunability, which is slightly larger than the demonstrated by current paraelectrically tuned filters [87]. The measurements show that the tuning range is center on the corner of the application range. This is resulted-in by the short and long surface non-planarities 
which is impact the capacity of the piezoelectric actuator significantly. Nevertheless, the represented tunable filter shows successfully a broad band application, i. e.: tunable frequency range of $1.5 \mathrm{GHz}$ with the application of $200 \mathrm{~V}$ control voltage.

\section{measurements \& simulations}

Deviations between measured and simulated characteristics are attributed to a slight angular misalignment of the cantilever in the plane of the LTCC surface. With values 4 to $2 \mathrm{~dB}$ the insertion loss at center frequency is relatively low due to the absence of lossy paraelectric material. The increase in the insertion loss at the lower end of tuning range, is due to the decrease in the overall resonator quality factor as the electrical length of the transmission line portion becomes shorter.

The simulations include resistive and dielectric losses; they particularly reveal resistive losses in the thin cantilever metallization as a major contribution. The predicted tuning range is from $0.8 \mathrm{GHz}$ to $2.5 \mathrm{GHz}$, tunability of $(250 \%)$. The different with the measured tunability range $(135 \%)$ can be attributed to the unintentional air gap (of $2.2 \mu \mathrm{m}$ ) in the capacitor present at zero bias where ideally the top electrode should touch the high-K dielectric layer. Due to that the effective dielectric constant between the capacitor electrodes has been dropped from its maximum value of 65 to 4.4 at zero-bias. Such an air gap likely arises due to the roughness and non-planarity of both LTCC and the piezoelectric actuator surfaces.

Like in MEMS devices, the capacitance variation is due to movement of the electrodes and not due to change in material parameters. Like in MEMS devices also, piezoelectrically tuned capacitors exhibit a high quality factor.

\section{roughnesses \& biasing capacitors}

The tuning range achievable with the present concept depends on several factors, particularly short and long range surface nonplanarities, i.e. roughness and warpage. As a practical design rule we found a minimum effective air gap of $1 \mu \mathrm{m}$ to account for residual surface roughness effects after polishing. The analysis of the device by electromagnetic simulation reveals a potential for $250 \%$ tuning range if roughness and warpage could be eliminated. The need for a large capacitors that allow biasing like what has been done in [88] which reduced the tuning range is not needed in this type of filter, since the applied bias is not affecting the RF behaviour of the device.

\section{attenuation}

However, lower side band attenuation deteriorates due to the lower pole of the filter remaining at fixed frequency since it is a zero frequency pole. This transmission zero is an inherent characteristic of this type of filter. It can be observed that the passband frequency response is flat and the frequency skirts are not sharp. This is attributed to the absence of a multipoles in the vicinity of the midband.

In the fabricated second-order filter, the attenuation has been improved by 10 to $15 \mathrm{~dB}$. A better selectivity has been achieved. From the measured filter, the performance of frequency 
skirt enhances the isolation.

For better attenuation, another pair of extra transmission zeros that can be easily tuned could be added by using other filter topology. The attenuation characteristics could be improved by inserting several poles in the lower/upper band of the center frequency of the filter by using a capacitive coupling between the resonators.

\section{stiction \& pull-in}

In [3] a piezoelectric-transducer (PET) controlled tunable bandpass filter has been demonstrated. A dielectric perturber has been attached to the PET. The movement of the PET perturbs the electromagnetic fields of the filter resonator line. This change the line capacitance and consequently the center frequency of the filter. A wide tuning range of $24 \%$ was achieved near $10 \mathrm{GHz}$ with little performance degradations. Contrasting that, the tuning ratio was not limited due to the so-called pull-in effect. Piezoelectric actuators exhibit proven reliability and life time. Sticking of the contact surfaces is not an issue due to the piezoelectric force.

\section{actuation voltage}

Although the current piezoceramic bimorph design with its capability to enforce downwards bending under reversed bias is already useful to compensate for both, the piezoceramic hysteresis and an unintentional air gap due to imperfect assembly, the response to bias voltage may be further improved by using more complex actuators like multilayer piezoceramic versions. Two multilayer bending actuators are available at [72] the first actuator has the following specifications: layer thickness of $48 \mu \mathrm{m}$ and operating voltage of 92 volts for the maximum deflection of $300 \mu \mathrm{m}$. The second has a layer thickness of $17 \mu \mathrm{m}$ and operating voltage of 30 volts for the maximum deflection of $0.7 \mathrm{~mm}$.

\section{future work}

Suggestions on how to further develop and improve the device are also given, concerning in particular:

1. the power handling capability for base station applications,

2. the linearity at higher signal amplitudes (IP3) and performance in an RF circuit,

3. the tuning speed and hysteresis,

4. the production feasibility and assembly process of the two ceramics,

5. yield, lifetime and fatigue. 


\subsection{Piezoelectric Tuning Properties}

After the successfully demonstrated a compact tunable filter using integrated piezoelectric LTCC varactor. These initial results show the potential of this new filter technology to produce low loss wide-tunable components. The mechanical properties of the piezoelectric actuator affects the performance of such type of tunable components. In the following sub-sections the tunable coupling, hysteresis behaviour, tuning sensitivity, power consumptions, and tuning speed are discussed.

\subsubsection{Tunable Coupling}

The movement of the piezoelectric actuator changes the spacing between the lines (Fig. 5.1). This change yields a tunable coupling. The spacing between the lines is $\sqrt{S^{2}+d^{2}}$, where $\mathrm{S}$ is the spacing between the lines at zero bias (in the ideal case, $d=0$ ). With increasing the air

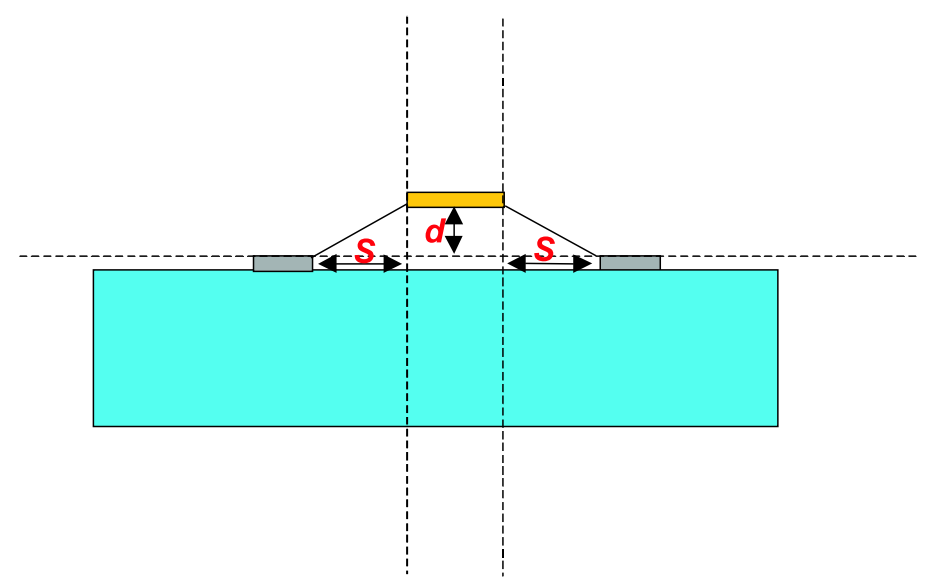

Figure 5.1.: Device cross-section.

gap the spacing between the lines increased, thus resulting in a more weaker coupling. Weak couplings between resonators are required for narrow-band filters while maintaining relatively small spacings between resonators. This is important because in the case of many microstrip resonator structures, in order to achieve narrow bandwidth, quite large spacings between resonators are required. By tunable coupling the isolation band has been improved at higher frequencies relative to that generated from the fixed, like in [2]. Also, it is one of the means for maintaining nearly constant bandwidth and passband shape since it is desired for the tunable filters over a very sizable range.

\subsubsection{Hysteresis Behaviour}

In general the dielectric hysteresis is an effect in a dielectric material similar to the hysteresis found in a magnetic material. It is the result of changes in orientation of electron orbits in the dielectric because of the rapid reversals of the polarity of the line voltage. The hysteresis in the 


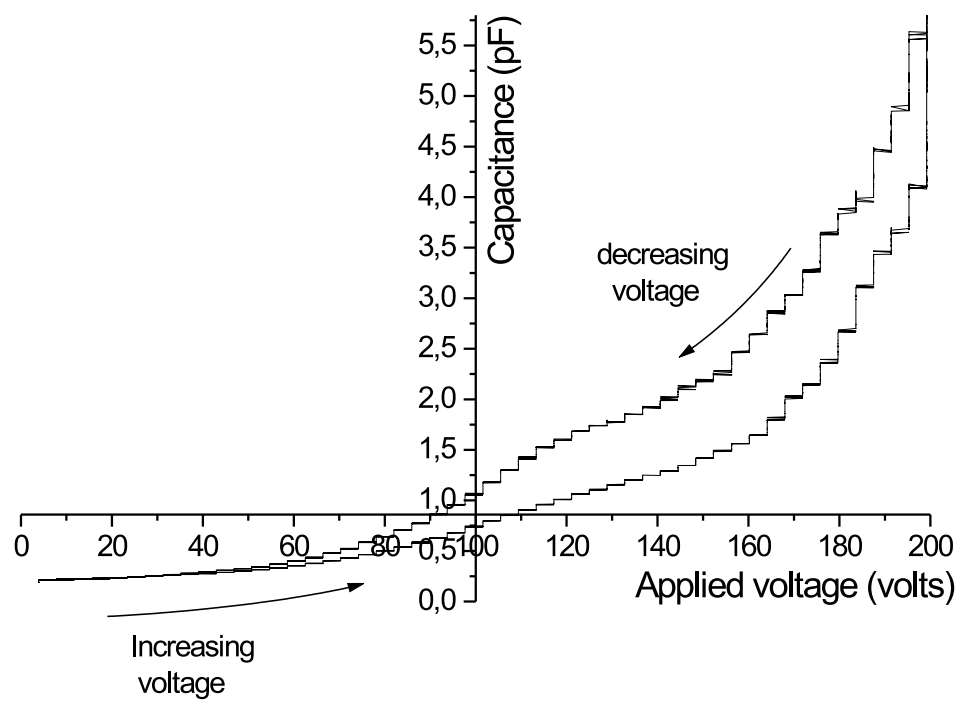

Figure 5.2.: Hysteresis in capacitance as a function of the applied voltage.

piezoelectric LTCC varactor is due to the dielectric-hysteresis of the PZT material from which the piezoelectric cantilever has been made. This could be controlled by driving the cantilever with a control circuit. Based on the mechanical measurements of the piezoelectric actuator glued to the LTCC, the hysteresis effect in the capacitance has been computed directly, and is shown in Fig. 5.2.

\subsubsection{Tuning Sensitivity}

To explore the tuning sensitivity of the fabricated device, we must analyze whether it is possible to pull the center frequency of the filter with the variable piezoelectric capacitor.

In order to achieve resonance, the reactance of the transmission line and the varactor must cancel. In the lumped equivalent circuit, the resonance occurs when the reactance of the inductor balances the reactance of the capacitor. The resonance frequency for the series $L C-$ tank is,

$$
f=\frac{1}{2 \pi \sqrt{L_{2} C}}
$$

To explore the change of frequency as a function capacitance, the partial differential equation is found to be:

$$
\frac{\partial f}{\partial C}=\frac{-f}{2 C}
$$

The tuning of this device is mainly limited by the range of the capacitance variation of the loaded varactor. From the equation (5) it is evident that the absolute tuning frequency range is proportional to the $\sqrt{C}$. 
For this topology, the tuning sensitivity versus the bias voltage is determined by

$$
\frac{\partial f}{\partial V}=\frac{\partial f}{\partial C} \frac{\partial C}{\partial V}
$$

and the change in the capacitance in terms of voltage change is:

$$
\frac{\partial C}{\partial V}=\frac{-C}{\left(d_{a i r}+\frac{d_{K}}{\epsilon_{r}}\right)} \frac{\partial d_{a i r}}{\partial V}
$$

where

$$
\frac{\partial d_{\text {air }}}{\partial V}=3 d_{31}\left(\frac{L}{t}\right)^{2}=\text { constant }
$$

Finally the tuning sensitivity is

$$
\frac{\partial f}{\partial V}=\frac{f}{2\left(d_{a i r}+\frac{d_{K}}{\epsilon_{r}}\right)} \frac{\partial d_{a i r}}{\partial V}
$$

since $d_{K} / \epsilon_{r}$ is small it could be ignored. Equation (10) become

$$
\frac{\partial f}{\partial V}=\frac{f}{2 V} \frac{G H z}{\text { Volts }}
$$

Fig. 5.3 shows the measured tuning sensitivity versus the applied DC voltage. The sensitivity decreases parabolically with increasing bias. A better sensitivity of tuning could be achieved by employing a multilayer actuator technology where a low bias voltages are needed verifying the fact that maximum sensitivity is achieved for minimum bias voltage. The sensitivity parameter is important for fine tuning the filter for the required center frequency with as minimal an applied electric filed/bias as possible. Since the PZT are nonlinear dielectric materials, the sensitivity factor will also be varying in a nonlinear fashion. Knowing the center frequency or the frequency shift versus the maximum applied electric filed would help in fine tuning the filter's operation.

\subsubsection{Power Consumptions}

Piezo-actuators operate as capacitive loads. Since the current leakage rate of the ceramic material is very low (resistance typically $10 \mathrm{M} \Omega$ ), piezo actuators consume almost no energy in a static application and therefore produce virtually no heat. As in electrostatically driven MEMS, an ideal piezoceramic element exchanges energy with the external circuit when mechanical movements occurs. This energy is not dissipated but an amount $\frac{1}{2} C V^{2}$ is stored in the electric field in the ceramic layer (capacitance $\mathrm{C}$, applied voltage $\mathrm{V}$ ). In a bimorph design there are 


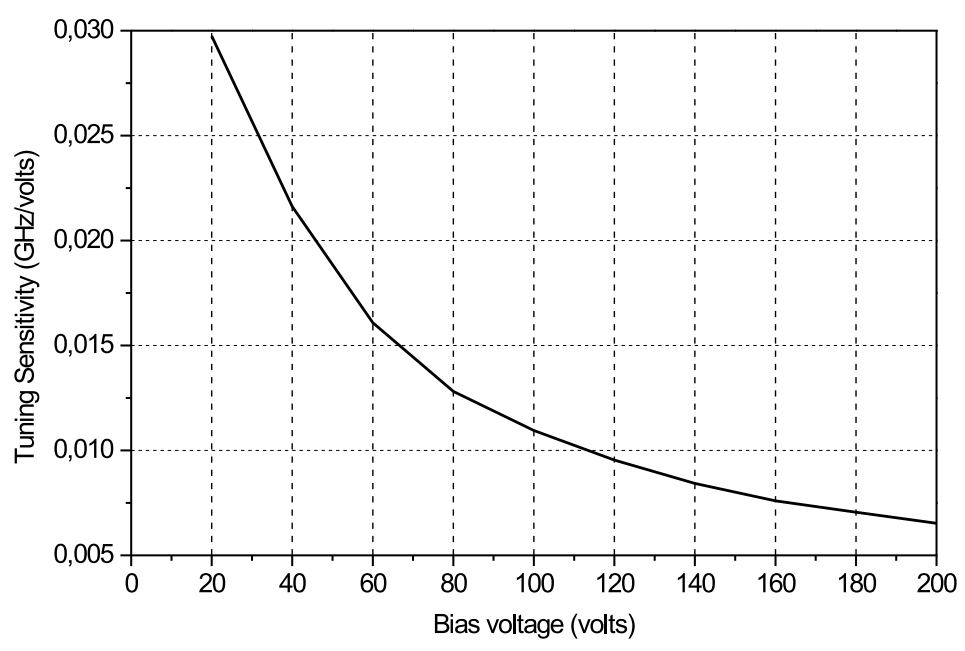

Figure 5.3.: Tuning sensitivity in frequency versus control voltage.

two piezoceramic layers with two outer electrodes and one inner electrode. The outer ones are kept on ground potential and maximum operation voltage $V_{\max }$, while the inner potential $V_{t}$ is tuned between these two values to control the bending. For $V_{t}=V_{\max } / 2$ the voltage drop across both piezoceramic layers is equal and no bending occurs. The maximum deflection in either direction is consequently induced for $V_{t}=0$ and $V_{t}=V_{\max }$, respectively.

The electrostatic energy can be expressed by

$$
E=\frac{1}{4} C V_{\max }^{2}+C\left(V_{t}-\frac{1}{2} V_{\max }\right)^{2}
$$

where $\mathrm{C}$ is the capacitance of each of the two layers. The maximal change per tuning step is given by

$$
\frac{-1}{4} C V_{\max }^{2}<\Delta E<\frac{1}{4} C V_{\max }^{2}
$$

where the sign denotes whether energy is stored or released. With an average permittivity of 3500 for the piezoelectric material VIBIRT 1334 [72], C amounts to $1.7 \mathrm{nF}$ and $\Delta E<3 \mu J$, which is comparable to MEMS switches [89]. Additional dissipative losses in conducting and ceramic materials are more difficult to assess and necessitate further experiments.

\subsubsection{Tuning Speed}

The size of the cantilever result in a small capacitance and relatively slow response time. The switching time depends on the sound velocity of the piezo material [90]. For the piezoelectric material VIBIRT 1334 the time response amounts to $2.43 \mathrm{~ms}$, to reach the fully open position, 
which is much slower than the switching speed of a semiconductor switch which is in the order of nanoseconds. The ferroelectric can be tuned rather fast 1 ps. Smaller size can be used to make more compact filter and improve the tuning time. Typical piezoelectric actuator can work up to about 1 billion cycles. Comparing this with the almost infinite working cycle for a semiconductor switch, piezo-actuators have shorter lifetimes.

\subsection{Competing Technologies}

Finally, to show the capabilities and the potential of tunable devices based on integrated piezoelectric LTCC varactor, it is important to compare the present approach with the competing technologies. Particularly the semiconductor varactor diodes, paraelectric capacitors, and MEMS capacitors. York et al. have compared the above mentioned continuously variable

Table 5.1.: Comparison of varactor technologies.

\begin{tabular}{|l|l|l|l|l|}
\hline & GaAs & BST & MEMS & $\begin{array}{l}\text { Piezoceramic } \\
\text { (This work) }\end{array}$ \\
\hline Tuning Range & $4: 1$ & $3: 1$ & $<1.5: 1$ & $5: 1$ \\
\hline Quality Factor & $<60$ & $<60$ & $<200$ & $<150$ \\
\hline Control Voltage & $<20 \mathrm{~V}$ & $<30 \mathrm{~V}$ & $<50-100 \mathrm{~V}$ & $<200 \mathrm{~V}$ \\
\hline Tuning Speed & Fast & Fast & Slow & Slow \\
\hline
\end{tabular}

varactor technologies in [7]. Including the present approach, four technologies for integrated microwave varactors exist. Some important features are summarized in Table 5.1. 


\section{Outlook-Future work}

This chapter presents an outlook for the PTF. The idea of the present approach that has been explained in the third chapter could be extended to tune multiple capacitors with a single cantilever. The filter topology plays the most important role in doing that. The major challenge would be where to place the capacitors with in the filter structure for optimum tunability. In higher-order version of the present structure (combline filter) a set of cantilevers will be driven simultaneously and individually to tune the filter. Thus, a complex control circuit design is required. The wish is to have a minimum number of cantilevers that can tune the maximum possible number of capacitors. Throughout this chapter a proposed solution which looks feasible will be demonstrated in simulations. Also, the angular misalignment, reproducibility, reliability, and fatigue are shortly addressed.

\subsection{Multiple tuned capacitances}

The present approach can be extended to tune more than only one capacitor simultaneously with one movable cantilever. As shown in Fig. 6.1, the configuration consists of a set of fixed

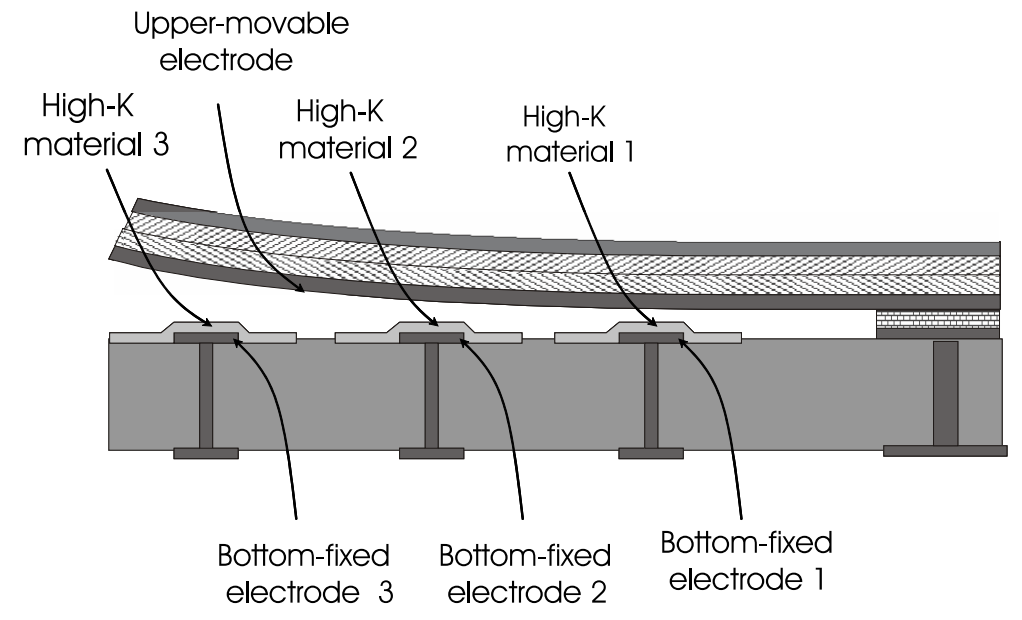

Figure 6.1.: Schematic of the piezoelectric tuned multi capacitors.

electrodes which are comprised in the top of a LTCC surface. Above each, a high-K material of different thicknesses with different dielectric constants may be used to form the intermediate layers. The whole set of the capacitors could be tuned by changing the distance between theses fixed electrodes by a single upper-movable electrode which could be raised by a piezoelectric actuator. Thus, the whole setup could be tuned by a single driving voltage. 


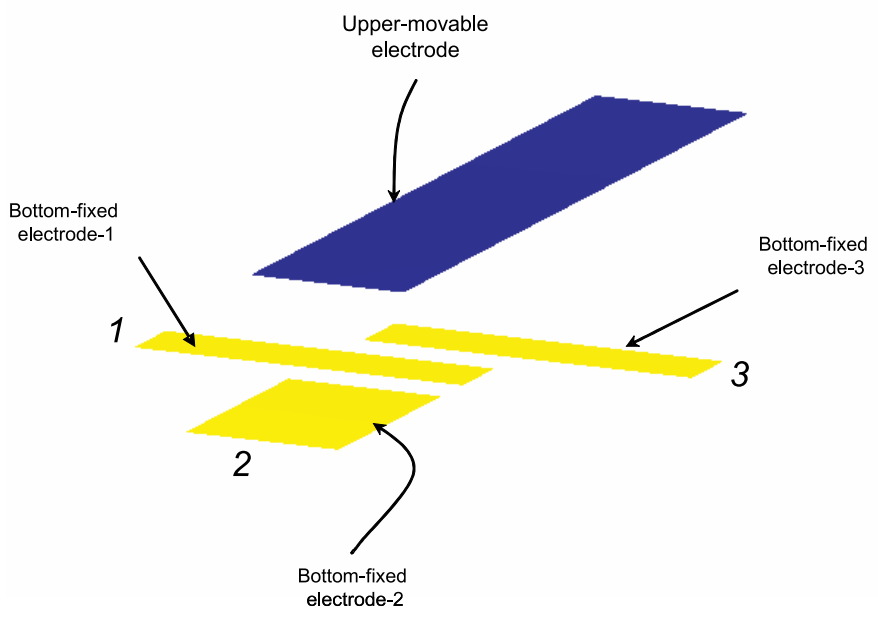

Figure 6.2.: 3D-view of the proposed-configuration.

The 3D-view of the proposed-configuration is shown in Fig. 6.2. The tuning device is composed of three capacitors which are simultaneously tuned by one movable electrode. The set of the fixed bottom electrodes 1,2 and 3 are located on the top of LTCC substrate. A high-K dielectric layer is sitting on the top of these electrodes. This three capacitor tuning method could be tuned with one applied voltage. The parasitics due to the coupling within this arrangement have to be taken in the design consideration. This setup has replaced multiple piezoelectric actuators with only one, as a result low insertion loss is to be expected.

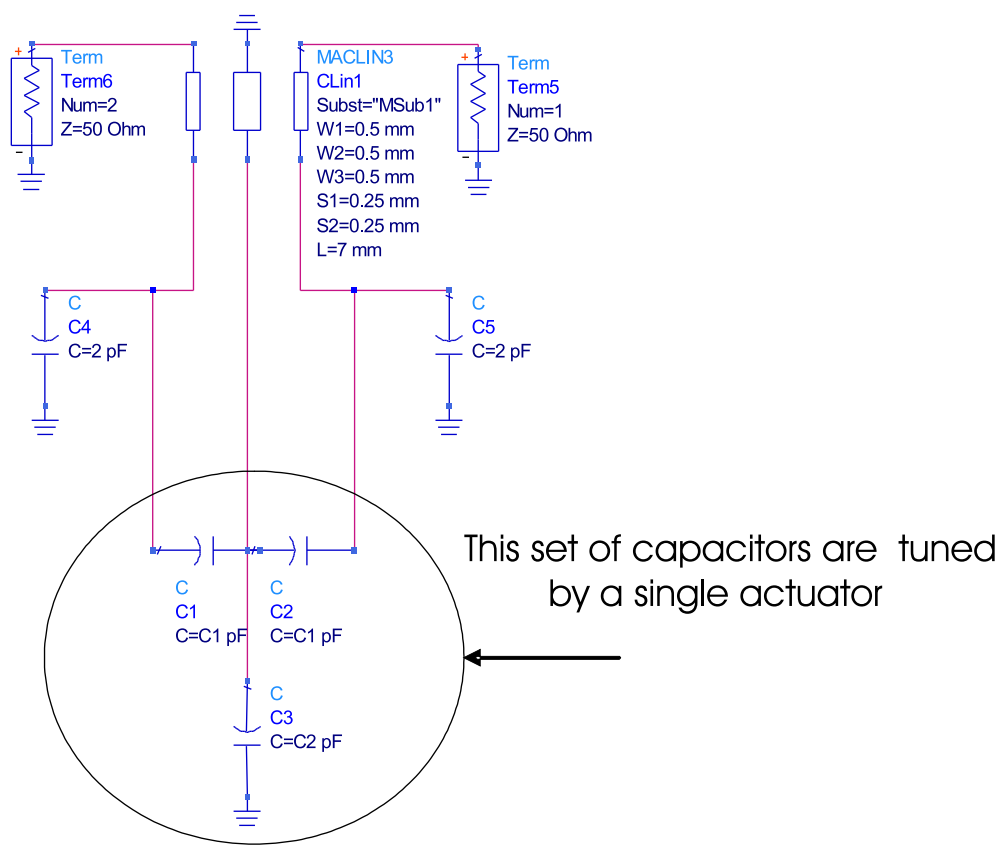

Figure 6.3.: Device outlook circuit. 
To illustrates the present concept, a proposed circuit is shown in Fig. 6.3 is chosen as demonstrator. The circuit employing three coupled lines. The two exterior lines used as input and output lines. The center coupled line is identical to the bottom metallization of the cantilever. The lower three capacitors $C_{1}, C_{2}, C_{3}$ are tunable. Fig. 6.4 shows the proposed cross-section for

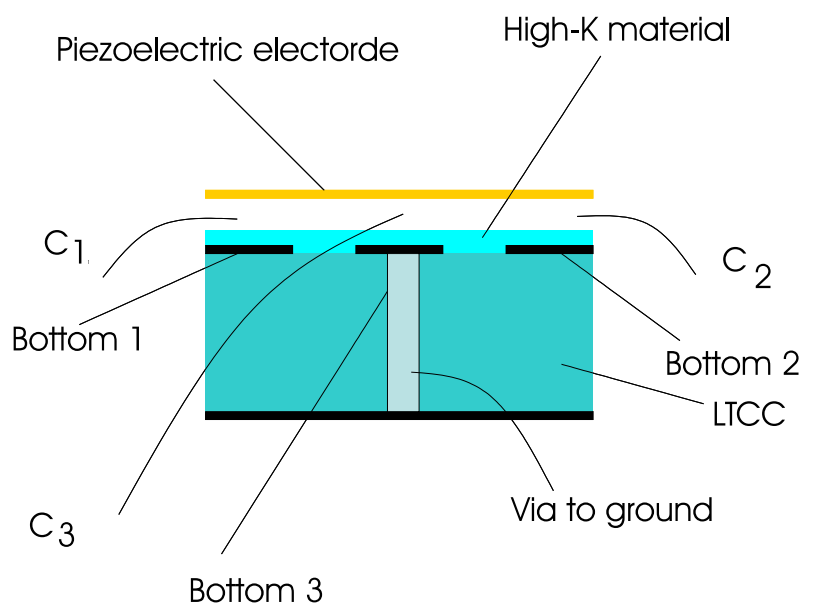

Figure 6.4.: Multi-tuned capacitors with single cantilever.

this structure where a single cantilever could be used to tune them all by single action (applied voltage). The bottom electrodes are set on the top of LTCC substrate. The distance between these electrodes could be adjusted as small as possible to make sure that the whole set will be covered by the width the cantilever. A high-K dielectric tape set on the top of these electrodes. The movement of the cantilever change the air gap inside the three capacitors simultaneously, consequently the capacitors varied and the filter characteristic is tuned. The circuit simulation using ADS is shown in Fig. 6.5. Where the three capacitors are identical to each other and were they tuned from $15 \mathrm{pF}$ to $1 \mathrm{pF}$. Fig. 6.5 shows that the attenuation on both the upper and the

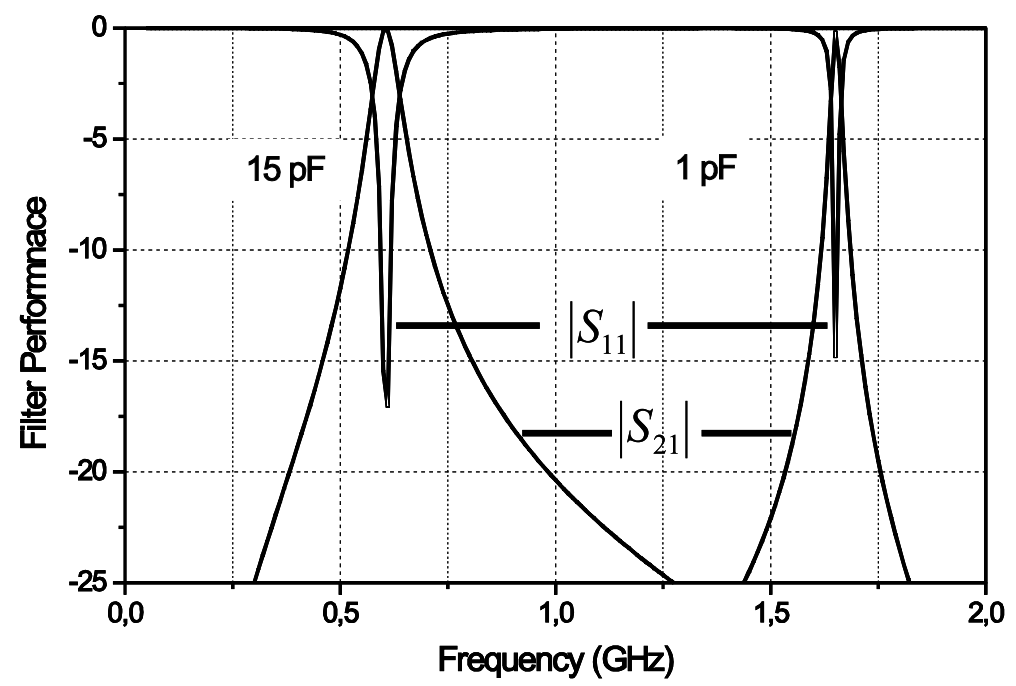

Figure 6.5.: Outlook: device performance. 
lower side has been improved. The two capacitors $C_{1}$ and $C_{2}$ which are terminating the input and the output resonators, respectively, introduce poles at the lower side of the transmission curve. These poles moved up in frequency as the two capacitors values decrease. These capacitors also change the cross-coupling between the coupled lines as consequent the bandwidth of the filter decreases with frequency goes up. There is no optimization has been done in terms of matching or losses.

\subsection{Angular Misalignment}

Since the misalignment of the cantilever horizontally and vertically is a critical issue, the cantilevers could be aligned parallel to the other lines by using a mask. Fig. 6.6 shows a schematic

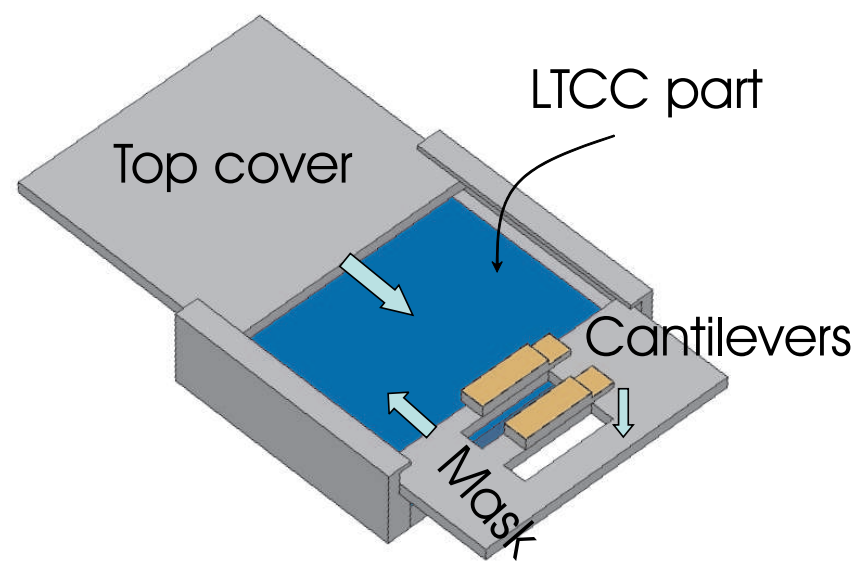

Figure 6.6.: Schematic of the fixing procedure using mask.

view for that, a U-shaped box is used to hold the LTCC-part of the device, an upper movable mask which might has a number of windows depending on the order of the filter used to hold the cantilevers inside. A top-cover is used to fix the cantilevers after flipping the whole chock for injecting the glue from the other side.

\subsection{Reproducibility, Reliability, and Fatigue}

This solution with mechanical tuning of a capacitor driven by piezoelectric effect looks feasible, however, the question of the reproducibility of the tuning characteristics and long-term stability of those characteristics has to be considered. The development and the fabrication may require considerable technological effort. 
Knowledge of mechanical properties is essential for the successful modeling and design of Electro Mechanical Systems. Fatigue behavior, for instance, may ultimately limit product lifetime in certain applications such as tunable components. Fatigue is of particular interest in tunable filters which operate under cyclic loading at high frequencies $(\mathrm{GHz})$. Failure under cyclic loading will depend on the magnitude of the applied strain. Like in MEMS structures which have been cycled billions of times without showing fatigue failure, I expect the same for this case.

The reliability, efficiency and improvement of such a device strongly depend on the characteristics of the thick film layer of the PZT material. The integration of piezoelectric actuator and LTCC technologies is emerging for the next wireless generation components, since the huge movement/displacement that could be achieved by a piezo-actuator is the key for a huge range of tunability. 



\section{Conclusion}

Integrated piezoelectrically tuned LTCC bandpass filters based on coupled microstrip lines have been investigated. Filter tuning is achieved by surface-mounted piezoceramic cantilevers. This work describes design, fabrication and experimental investigation of the tunable LTCC filters. The present approach offers innovative tunability performance that combines favorable properties with respect to tuning range, quality factor and power consumption.

- Similar to the known micro-electromechanical (MEMS) approaches for tuning and switching, in this work a modified parallel plate capacitor with high-permittivity dielectric and piezoelectrically movable top electrode is proposed as a promising and versatile tuning element. The vertical movement of the electrode controls the width of an air gap above the dielectric film. By this way the use of lossy dielectric material in the tuning element is avoided.

- This varactor has been integrated with the bandpass filter structure employing three coupled microstrip lines. One electrode of the piezoelectric element replaces one section of the center coupled resonator line of the conventional untuned filter so that the tuning function consumes no extra space. By splitting the center line into two sections and inserting the capacitor at the center of this line, the optimum tunability has been achieved.

- A systematic design procedure to design wideband tunable microstrip combline filters has been presented. The proposed method yields minimum change in the filter specification over the tuning range. This is achieved by considering the effects of tuning in the filter design.

- Since the bottom metallization of the actuator has been utilized as a part of the device, its parabolic bending will create a nonuniform air gap that varies from zero at the clamping point to several microns at the tip which result in very high aspect ratios and inadequately large if not prohibitive number of cells for correct simulations. To simplify the simulation, the bottom side of the cantilever is replaced by a straight line while the air gap inside the structure is replaced by an effective uniform layer.

- Small air gaps cause large frequency shift. Therefor a smooth surface is required. To achieve this, the fabrication has to fulfil requirements in substrate polishing and cantilever assembly. The minimum frequency at zero bias will otherwise be shifted up, thus reducing the tuning range. In order to guarantee optimum performance of both, the LTCC integrated coupled line filter elements and the piezoelectric element, at hybrid assembly process of two separately fabricated and optimized components is adopted here. A hybrid integration and assembly of the two components has been used, a conductive epoxy 
has been used to fix the cantilever in its position. The glue must have good mechanical properties and to provide a good electrical conductivity

- A preliminary demonstrator exhibits a wide tuning range from 1.1 to $2.6 \mathrm{GHz}$ with low insertion loss values of $3 \pm 1 \mathrm{~dB}$ throughout the tuning range. These promise further reduced insertion losses by thicker metallizations as well as even wider tuning range with tighter control of the surface roughness inside the capacitor. The tuning voltage of up to $200 \mathrm{~V}$ may be reduced in future versions by using multilayer actuators. The capacitor exhibits a wide tuning range from $7 \mathrm{pF}$ to $1.35 \mathrm{pF}$ with a quality factor between 60 and 160. A second-order filter has been fabricated to improve the selectivity and the attenuation. The filter has shown a better selectivity of 10 to $15 \mathrm{~dB}$ more than the first-order. The quality factor of the capacitor has been improved by a factor of 2 for a cantilever metallization of $150 \mathrm{~nm}$ gold.

- Like in MEMS devices, the capacitance variation is due to movement of the electrodes and not due to change in material parameters. It is useful for a broadband of operation from $0.1 \mathrm{GHz}$ to $100 \mathrm{GHz}$. Piezoelectric actuators exhibit proven reliability and life time. Sticking of the contact surfaces is not an issue due to the piezoelectric force. The tuning ratio was not limited due to the so-called pull-in effect.

- To reduce the size and weight of the filter, a high dielectric constant could be used as a substrate and more thinner PZT layer in the piezoelectric actuator. Another filter topology might be used to improve the selectivity and higher the quality factor of the filter, i.e., tapped input/output combline filter, where a two extra zeros can be added to over come the lower-side attenuation which is caused by the fixed zero-pole.

- The tuning range achievable with the present concept depends on several factors, particularly short and long range surface nonplanarities, i.e. roughness and warpage. As a practical design rule we found a minimum effective air gap of $1 \mu \mathrm{m}$ to account for residual surface roughness effects after polishing. The analysis of the device by electromagnetic simulation reveals a potential for $250 \%$ tuning range if roughness and warpage could be eliminated.

- The sensitivity parameter is important for fine tuning the filter for the required center frequency with as minimal an applied electric filed/bias as possible. Since the PZT are nonlinear dielectric materials, the sensitivity factor will also be varying in a nonlinear fashion. Knowing the center frequency or the frequency shift versus the maximum applied electric filed would help in fine tuning the filter's operation. The size of the cantilever result in a small capacitance and relatively slow response time. The switching time depends on the sound velocity of the piezo material. For the piezoelectric material VIBIRT 1334 the time response amounts to $2.43 \mathrm{~ms}$, to reach the fully open position, which is much slower than the switching speed of a semiconductor switch which is in the order of nanoseconds. The power consumption $\Delta E<3 \mu \mathrm{J}$, which is comparable to MEMS switches. Additional dissipative losses in conducting and ceramic materials are more difficult to assess and necessitate further experiments. 
- The present approach can be extended to tune more than only one capacitor simultaneously with single movable cantilever. The tuning device might be composed of a set of fixed bottom electrodes which are located on the top of LTCC substrate. A high-K dielectric layer is sitting on the top of these electrodes. The whole set could be tuned with one applied voltage. The parasitics due to the coupling within this arrangement have to be taken in the design consideration. This setup has replaced multiple piezoelectric actuators with only one, and a low loss is to be expected.

This tunable device can be successfully used in applications where the large tuning range and the very effective integration of the 3D LTCC packages are much more important than the very slow speed. 



\section{A. Two-Port Parameters}

Consider the 2-port shown in Fig. A.1. In the impedance matrix representation, the voltage at each port is related to the currents at the different ports as follow:

$$
\underline{\mathrm{V}}=\mathbf{z} \underline{\mathbf{I}}
$$

where $\mathbf{z}$ is the impedance matrix and $\underline{\mathbf{V}}$ and $\underline{\mathbf{I}}$ are the voltage and current vectors.

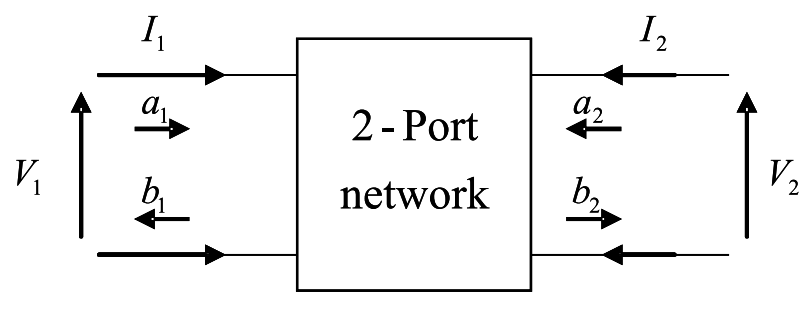

Figure A.1.: 2-port network parameters definition.

$$
\underline{\mathbf{V}}=\left[\frac{V_{1}}{V_{2}}\right] \quad \underline{\mathbf{I}}=\left[\frac{I_{1}}{I_{2}}\right]
$$

and

$$
\mathbf{z}=\frac{1}{Z_{0}}\left[\begin{array}{ll}
Z_{11} & Z_{12} \\
Z_{21} & Z_{22}
\end{array}\right]=\left[\begin{array}{ll}
z_{11} & z_{12} \\
z_{21} & z_{22}
\end{array}\right]
$$

where $Z_{0}$ is the characteristic impedance. A very popular method of representing microwave networks is by the scattering matrix. The scattering matrix is generally represented in a normalized form. In this representation, the normalized reflected voltage at each port of the network is related to the normalized incident voltages at the ports of the networks. The scattering matrix of the two port network relates the reflected waves $\underline{b_{1}}, \underline{b_{2}}$ and the incident waves $\underline{a_{1}}, \underline{a_{2}}$ at the input and the output ports. It is defined as follow:

$$
\underline{\mathbf{b}}=\mathbf{S} \underline{\mathbf{a}}
$$

where 


$$
\underline{\mathbf{b}}=\left[\begin{array}{l}
\frac{b_{1}}{b_{2}} \\
\underline{\mathbf{a}}
\end{array}\right] \quad\left[\begin{array}{l}
\frac{a_{1}}{a_{2}}
\end{array}\right]
$$

and

$$
\mathbf{S}=\left[\begin{array}{ll}
S_{11} & S_{12} \\
S_{21} & S_{22}
\end{array}\right]
$$

where $S_{11}$ and $S_{22}$ are called the input and the output reflection coefficients, respectively. $S_{12}$ is the reverse transmission coefficient and $S_{21}$ is the forward transmission coefficient. The relationships between the voltage and current with incident and reflected waves at the input output ports are defined as follow:

$$
\begin{aligned}
& \underline{\mathbf{V}}=\underline{\mathbf{a}}+\underline{\mathbf{b}} \\
& \underline{\mathbf{I}}=\underline{\mathbf{a}}-\underline{\mathbf{b}}
\end{aligned}
$$

Substituting the voltage and current in terms of incident and reflected waves in the impedance matrix yields

$$
\underline{\mathbf{a}}+\underline{\mathbf{b}}=\mathbf{z} \underline{\mathbf{a}}-\underline{\mathbf{b}}
$$

since $\underline{\mathbf{b}}=\mathbf{S} \underline{\mathbf{a}}$, this implies that

$$
\underline{\mathbf{a}}+\mathrm{S} \underline{\mathbf{a}}=\mathrm{z}[\underline{\mathbf{a}}-\mathrm{S} \underline{\mathbf{a}}]
$$

that results in

$$
\mathbf{z}=[\mathbf{1}-\mathbf{S}]^{-1}[\mathbf{1}+\mathbf{S}]
$$

where

$$
\mathbf{1}=\left[\begin{array}{ll}
1 & 0 \\
0 & 1
\end{array}\right]
$$

The input impedance at the input port can be computed as follow:

$$
Z_{\text {in }}=Z_{0} \frac{V_{1}}{\underline{I_{1}}}=Z_{0} \frac{\underline{a_{1}}+\underline{b_{1}}}{\underline{a_{1}}-\underline{b_{1}}}
$$


with

$$
\underline{b_{1}}=S_{11} \underline{a_{1}}
$$

SO

$$
Z_{\text {in }}=Z_{0} \frac{1+S_{11}}{1-S_{11}}
$$

Since the two-port network is passive one can obtain

$$
0 \leq\left|S_{12}(\mathrm{j} \omega)\right|^{2} \leq 1, \quad\left|S_{11}(\mathrm{j} \omega)\right| \leq 1
$$

this stating conservation of energy, that a passive device cannot reflect more energy than the incident upon it. If the network is lossless then all the incident energy must be reflected from the network, i.e.

$$
\left|S_{11}(\mathrm{j} \omega)\right|=1
$$

In general the elements of a normalized scattering matrix satisfy the following equation, which results from the law of conservation of power [91]

$$
\mathbf{S}^{\dagger} \mathbf{S}=\mathbf{1}
$$

where $^{\dagger}$ denotes the Hermitian conjugate. Thus, $[\mathbf{1}]$ is called a unitary matrix. For two-port networks this yields the following identities

$$
\begin{gathered}
\left|S_{11}(\mathrm{j} \omega)\right|^{2}+\left|S_{12}(\mathrm{j} \omega)\right|^{2}=1 \\
\left|S_{22}(\mathrm{j} \omega)\right|^{2}+\left|S_{12}(\mathrm{j} \omega)\right|^{2}=1 \\
S_{11}(\mathrm{j} \omega) S_{12}{ }^{*}(\mathrm{j} \omega)+S_{12}(\mathrm{j} \omega) S_{22}{ }^{*}(\mathrm{j} \omega)=0
\end{gathered}
$$

The solution of the above set of equations leads to the following conclusions:

$$
\begin{aligned}
& \left|S_{11}(\mathrm{j} \omega)\right|=\left|S_{22}(\mathrm{j} \omega)\right| \\
& \left|S_{12}(\mathrm{j} \omega)\right|=\left|S_{21}(\mathrm{j} \omega)\right|
\end{aligned}
$$


In general and for $N$-port network, the following two equations relate the scattering matrix in terms of the impedance matrix, and vice versa are

$$
\mathbf{S}=[\mathbf{z}+\mathbf{U}]^{-1}[\mathbf{z}-\mathbf{U}]
$$

and

$$
\mathbf{z}=[\mathbf{U}-\mathbf{S}]^{-1}[\mathbf{U}+\mathbf{S}]
$$

where $\mathbf{U}$ is the unit, or identity matrix defined as

$$
\mathbf{U}=\left[\begin{array}{llllll}
1 & 0 & & & 0 \\
0 & 1 & 0 & & 0 \\
0 & 0 & 1 & & \\
& & & & \\
& & & 1 & 0 \\
0 & 0 & 0 & & 1
\end{array}\right]
$$




\section{Bibliography}

[1] J. Uher and W. Hoefer, "Tunable microwave and millimeter-wave band-pass filters," IEEE Transactions on Microwave Theory and Techniques, vol. 39, no. 4, pp. 643-653, April 1991.

[2] A. Tombak, J. P. Maria, F. Ayguavives, J. Zhang, G. Stauf, A. Kingon, and A. Mortazawi, "Voltage-controlled rf filters employing thin-film barium-strontium-titanate tunable capacitors," IEEE Transactions on Microwave Theory and Techniques, vol. 51, no. 2, pp. $462-$ 467, Feb. 2003.

[3] Y. Tae-Yeoul and C. Kai, "Piezoelectric-transducer-controlled tunable microwave circuits," IEEE Transactions on Microwave Theory and Techniques, vol. 50, no. 5, pp. 1303 - 1310, May 2002.

[4] M. Sanchez-Renedo, R. Gomez-Garcia, J. Alonso, and C. Briso-Rodriguez, "Tunable combline filter with continuous control of center frequency and bandwidth," IEEE Transactions on Microwave Theory and Techniques, vol. 53, no. 1, pp. 191-199, Jan. 2005.

[5] B. S. Virdee, "Current techniques for tuning dielectric resonators," Microwave Journal, vol. 46, no. 10, pp. 130-138, Oct. 1998.

[6] K. Kageyama, K. Satio, H. Utaki, and T. Yamamoto, "Tunable active filters having multilayer structure using ltcc," IEEE Transactions on Microwave Theory and Techniques, vol. 49, no. 12, pp. 2421-2424, Dec. 2001.

[7] R. York, A. Nagra, E. Erker, T. Taylor, P. Periaswamy, J. Speck, S. Striffer, D. Kaufmann, and O. Auciello, "Microwave integrated circuits using thin-film bst," IEEE International Symp. on Applications of Ferroelectrics (ISAF), vol. 1, pp. 195-200, July 2000.

[8] H. Nieminen, V. Ermolov, K. Nybergh, S. Silanto, and T. Ry-hnen, "Design of a tempreture-stable rf mems," Journal of Microelectromechani Systems, vol. 13, no. 5, pp. 795-714, Oct. 2004.

[9] J. Jantree, P. Akkaraekthalin, and V. Vivek, "An electronically tunable bandpass filter using a coplanar structure," in Int. Symp. On Communications and Information Tech., Chiangmai, Thailand, Nov. 2001, pp. 437-440.

[10] I. Lockerbie and S. Kumar, "A broadband tunable combline filter using active devices," in IEEE Conference Proceedings in Communications, Computers and Power in the Modern Environment, Chiangmai, Thailand, May 1993, pp. $196-200$. 
[11] K. Kageyama, K. Saito, H. Murase, H. Utaki, and T. Yamamoto, "Tunable active filters having multilayer structure using ltcc," in Proceedings of the IEEE MTT-S International Microwave Symposium Digest, vol. 3, Phoenix, AZ, May 2001, pp. 1445 - 1448.

[12] A. Tombak, J.-P. Maria, F. Ayguavives, J. Zhang, G. Stauf, A. Kingon, and A. Mortazawi, "Tunable barium strontium titanate thin film capacitors for $\mathrm{rf}$ and microwave applications," IEEE Microwave and Wireless Components Letters, vol. 12, no. 1, pp. 3-5, Jan. 2002.

[13] M. Ouaddari, S. Delprat, F. Vidal, M. Chaker, and W. Ke, "Microwave characterization of ferroelectric thin-film materials," IEEE Transactions on Microwave Theory and Techniques, vol. 53, no. 4, pp. 1390 - 1397, April 2005.

[14] R. Kurchania, A. Bell, T. Chakraborty, and I. Hunter, "An investigation of bst:mgtio/sub 3/ and x7r:mgtio/sub 3/ based ceramics for microwave applications," in IEEE International Symposium on Applications of Ferroelectrics, vol. 1, Montreal, Canada, Aug. 2004, pp. $281-284$.

[15] R. Jakoby, P. Scheele, S. Muller, and C. Weil, "Nonlinear dielectrics for tunable microwave components," in 15th International Conference on Microwaves, Radar and Wireless Communications, vol. 2, Warszawa, Poland, May 2004, pp. 369 - 378.

[16] G. Subramanyam, A. Zaman, N. Mohsina, F. Van Keuls, F. Miranda, and R. Romanofsky, "A narrow-band ferroelectric tunable bandpass filter for k-band applications," in $M i$ crowave Conference, 2000 Asia-Pacific, vol. 2, Sydney, NSW, Dec. 2000, pp. 938 - 941.

[17] L. Yu, A. Nagra, E. Erker, P. Periaswamy, T. Taylor, J. Speck, and R. York, "Basrtio3 interdigitated capacitors for distributed phase shifter applications," IEEE Microwave and Guided Wave Letters, vol. 10, no. 11, pp. 448 - 450, Nov. 2000.

[18] K. Dongsu, C. Yoonsu, M. Allen, J. Kenney, and D. Kiesling, "A wide bandwidth monolithic bst reflection-type phase shifter using a coplanar waveguide lange coupler," in IEEE MTT-S International Microwave Symposium Digest, vol. 3, Seattle, WA, June 2002, pp. 1471 - 1474.

[19] Paratek-Microwave, "Thin film electronically tunable pre-selectors for software defined radios," Microwave Journal, vol. 47, no. 10, pp. 138-144, Oct. 2004.

[20] M. Bakri-Kassem and R. Mansour, "An improved design for parallel plate mems variable capacitors," in IEEE MTT-S International Microwave Symposium Digest, vol. 2, Fortworth, Texas, June 2004, pp. 865 - 868.

[21] H. Wu, K. Harsh, R. Irwin, Z. Wenge, A. Mickelson, Y. Lee, and J. Dobsa, "Mems designed for tunable capacitors," in IEEE MTT-S International Microwave Symposium Digest, vol. 1, Batirnore, MD, June 1998, pp. 127 - 129. 
[22] F. Yang, "Membrane modeling of pull-in instability in mems sensors and actuators," in IEEE Sensors, vol. 2, Orlando, FL, June 2002, pp. 1199 - 1203.

[23] L. C. Wei, A. Mohammad, and N. Kassim, "Analytical modeling for determination of pull-in voltage for an electrostatic actuated mems cantilever beam," in IEEE International Conference on Semiconductor Electronics, Penang, Malaysia, Dec. 2002, pp. 233 - 238.

[24] L. Mercado, S.-M. Kuo, T.-Y. Lee, and L. Lianjun, "Mechanics-based solutions to rf mems switch stiction problem," IEEE Transactions on Components and Packaging Technologies, vol. 27, no. 3, pp. 560 - 567, Sept. 2004.

[25] A. Dec and K. Suyama, "Microwave mems-based voltage-controlled oscillators," IEEE Transactions on Microwave Theory and Techniques, vol. 48, no. 11, pp. 1943 - 1949, Nov. 2000.

[26] P. Chuan, P. Sangtae, P. Chu, L. Shi-Sheng, T. Ming, D. Peale, N. Bonadeo, and I. Brener, "Electrostatic actuation of three-dimensional mems mirrors using sidewall electrodes," IEEE Journal of Selected Topics in Quantum Electronics, vol. 10, no. 3, pp. 472 - 477, May 2004.

[27] L. Hee-Chul, P. Jae-Yeong, and B. Jong-Uk, "Piezoelectrically actuated rf mems dc contact switches with low voltage operation," IEEE Microwave and Wireless Components Letters, vol. 15, no. 4, pp. 202 - 204, April 2005.

[28] P. Robert, D. Saias, C. Billard, S. Boret, N. Sillon, C. Maeder-Pachurka, P. Charvet, G. Bouche, P. Ancey, and P. Berruyer, "Integrated rf-mems switch based on a combination of thermal and electrostatic actuation," in International Conference on TRANSDUCERS, Solid-State Sensors, Actuators and Microsystems, vol. 2, Boston, USA, June 2003, pp. $1714-1717$.

[29] F. Zhiping, Z. Wenge, S. Bingzhi, K. Harsh, K. Gupta, V. Bright, and Y. Lee, "Design and modeling of rf mems tunable capacitors using electro-thermal actuators," in IEEE MTT-S International Microwave Symposium Digest, vol. 4, Anaheim, CA, June 1999, pp. 1507 1510 .

[30] Y. Jun-Bo and C.-C. Nguyen, "A high-q tunable micromechanical capacitor with movable dielectric for rf applications," in IEDM Technical Digest. International Electron Devices Meeting, San Francsisco, CA, Dec. 2000, pp. 489 - 492.

[31] W. Cong-Shun, Y. Zhen-Chuan, C. Yun-Jun, F. Jing, and Z. Da-Cheng, "Design and fabrication of a novel two-dimension mems-based tunable capacitor," in IEEE International Conference on Communications, Circuits and Systems and West Sino Expositions, vol. 2, Chengdu, China, June 2002, pp. 1766 - 1769.

[32] Z. Jun, L. Chang, J. Schutt-Aine, C. Jinghong, and K. Sung-Mo, "Development of a wide tuning range mems tunable capacitor for wireless communication systems," in IEDM 
Technical Digest. International Electron Devices Meeting, San Francsisco, CA, Dec. 2000, pp. $403-406$.

[33] T. Rijks, J. van Beek, P. Steeneken, M. Ulenaers, J. De Coster, and R. Puers, "Rf mems tunable capacitors with large tuning ratio," in IEEE International Conference on Micro Electro Mechanical Systems, Maastricht, The Netherland, Dec. 2004, pp. 777 - 780.

[34] J. Park, Y. Yee, H. Nam, and J. Bu, "Micromachined rf mems tunable capacitors using piezoelectric actuators," in IEEE MTT-S International Microwave Symposium Digest, vol. 3, Phoenix, AZ, May 2001, pp. $2111-2114$.

[35] W. Tuttlebee, "Software-defined radio: facets of a developing technology," IEEE Wireless Communications, vol. 6, no. 2, pp. 38-44, April 1999.

[36] — Software defined radio. John Wiley \& Sons, Inc., 2002.

[37] C.-J. Wang and W.-T. Tsai, "A slot antenna module for switchable radiation patterns," IEEE Antennas and Wireless Propagation Letters, vol. 4, pp. 202 - 204, April 2005.

[38] H. Shitang, L. Honglang, L. Shunzhou, and X. Shu, "Low loss and high stopband rejection switchable saw filter bank," in IEEE Ultrasonics Symposium, vol. 1, Munich, Germany, Oct. 2002, pp. $193-196$.

[39] M. Sadiku and C. Akujuobi, "Software-defined radio: a brief overview," IEEE Potentials, vol. 23, no. 4, pp. 14-15, Oct. 2004.

[40] M. Rahman and S. Khosro, "Electronically tunable ltcc based multilayer filter for mobile handset applications," in IEEE MTT-S Int. Microwave Symp. Dig., vol. 3, Philadelphia, PA, June 2003, pp. 1767-1770.

[41] R. Levy, R. Snyder, and G. Matthaei, "Design of microwave filters," IEEE Transactions on Microwave Theory and Techniques, vol. 50, no. 3, pp. 783 - 793, March 2002.

[42] B. Y. Kapilevich, "Microwave active filter in integrated waveguide technology," Electronics Letters, vol. 32, no. 19, pp. 1789 - 1790, Sept. 1996.

[43] W. Harrison, "A miniature high-q bandpass filter employing dielectric resonators," IEEE Transactions on Microwave Theory and Techniques, vol. 16, no. 4, pp. 210 - 218, Apr. 1968.

[44] H. Jia-Sheng and M. Lancaster, "Couplings of microstrip square open-loop resonators for cross-coupled planar microwave filters," IEEE Transactions on Microwave Theory and Techniques, vol. 44, no. 11, pp. 2099 - 2109, Nov. 1996.

[45] I. Hunter, L. Billonet, B. Jarry, and P. Guillon, "Microwave filters-applications and technology," IEEE Transactions on Microwave Theory and Techniques, vol. 50, no. 3, pp. 794 - 805, March 2002. 
[46] H. Orchard and D. Targoff, "Image-parameter approximations for designing linear-phase filters," IEEE Transactions on Circuits and Systems, vol. 25, no. 6, pp. 325 - 333, Jun. 1976.

[47] G. Matthaei, "Design of wide-band (and narrow-band) band-pass microwave filters on the insertion loss basis," IEEE Transactions on Microwave Theory and Techniques, vol. 8, no. 6, pp. 580 - 593, Nov 1960.

[48] H. Daae, "Computer-aided optimal synthesis of bandpass filters," IEEE Transactions on Circuits and Systems, vol. 22, no. 6, pp. 490 - 495, Jun. 1975.

[49] H. N. Dawirs, "Graphical filter analysis," IEEE Transactions on Microwave Theory and Techniques, vol. 3, no. 1, pp. 15 - 21, Jan. 1955.

[50] R. Cameron, "General coupling matrix synthesis methods for chebyshev filtering functions," IEEE Transactions on Microwave Theory and Techniques, vol. 47, no. 4, pp. 433 442, Apr. 1999.

[51] H. Baher, Synthesis of electrical network. Wiley, N. Y., 1984.

[52] F. F. Kuo, Network analysis snd synthesis. Wiley, N. Y., 1966.

[53] M. E. Van Valkenburg, Introduction to network analysis. Wiley, N. Y., 1960.

[54] R. Gudipati and W.-K. Chen, "Explicit formulas for element values of the lowpass, highpass and bandpass ladders with butterworth or chebyshev response," in Proceedings of the 36th Midwest Symposium on Circuits and Systems, vol. 1, Detroit, MI, Aug. 1993, pp. 645 648.

[55] D. Baez-Lopez and E. Jimenez-Lopez, "A modified inverse-chebyshev filter with an all positive elements ladder passive realization," in Proceedings of the IEEE International Symposium on Circuits and Systems, vol. 6, Orland, FL, Jan. 1999, pp. 49 - 52.

[56] J. Jin-guang, H. Yi-gang, and W. Jie, "Design of fully differential fourth-order bessel filter with accurate group delay," in Proceedings of the International Conference on ASIC, vol. 1, Shanghai, China, Oct. 2001, pp. $281-284$.

[57] G. C. Temes and S. K. Mitra, Modern Filter Theory and Design. Wiley, N. Y., 1973.

[58] G. Matthaei, L. Young, and E. M. T. Jones, Microwave Filters, Impedance Matching Networks, and Coupling Structures. Artech House.

[59] I. Hunter, Theory and Design of Microwave Filters. London: IEE, 2001.

[60] W.-K. Chen, The Circuits and Filters Handbook. IEEE Prsess, N. Y., 1995.

[61] I. Bahl, Lumped Elements for RF and Microwave Circuits. Atech House, London, 2003. 
[62] M. Gmitrovic and Z. Milosavljevic, "Designing of filters by using kuroda's identities," in Proceedings of the 2th International Conference on Microwaves and Radar, vol. 1, Krakow, May 1998, pp. 13-17.

[63] Y. Nemoto, K. Satake, M.and Kobayashi, and R. Sato, "Equivalent transformations for the mixed lumped richards section and distributed transmission line," IEEE Transactions on Microwave Theory and Techniques, vol. 36, no. 4, pp. 635 - 641, Apr. 1988.

[64] A. Grayzel, "A synthesis procedure for transmission line networks," IRE Transactions on Circuit Theory, vol. 5, no. 3, pp. 172 - 181, Sep. 1958.

[65] S. Cohn, "Parallel-coupled transmission-line-resonator filters," IEEE Transactions on Microwave Theory and Techniques, vol. 6, no. 2, pp. 223 - 231, Apr. 1958.

[66] J. O. Scanlan, "Theory of microwave coupled-line network," in Proceedings of the IEEE, vol. 68, Krakow, Feb. 1980, pp. 209-231.

[67] W. Chi, K. Zaki, A. Atia, and T. Dolan, "Dielectric combline resonators and filters," IEEE Transactions on Microwave Theory and Techniques, vol. 46, no. 12, pp. 2501 - 2506, Dec. 1998.

[68] R. Saal and W. Entenmann, Handbuch zum Filterentwurf - Handbook of Filter Design. Heidelberg: Hüthig, 1988.

[69] I. C. Hunter and R. J. D., "Electronically tunable microwave bandpass filters," IEEE Transactions on Microwave Theory and Techniques, vol. 30, no. 9, pp. 1354 -1360, Sep. 1982.

[70] G. Torregrosa-Penalva, G. Lopez-Risueno, and J. I. Alonso, "A simple method to design wide-band electronically tunable combline filters," IEEE Transactions on Microwave Theory and Techniques, vol. 50, no. 1, pp. 172 - 177, Jan. 2002.

[71] M. Hrovat, J. Holc, S. Drnovek, D. Belavic, J. Bernard, M. Kosec, L. Golonka, A. Dziedzic, and J. Kita, "Characterization of pzt thick films fired on ltcc substrates," Journal. of materials science letters, vol. 22, pp. 1193-1195, June 2003.

[72] Argillon, "Vibrit from argillon," www.piezo-power.com.

[73] W.-H. Chu, M. Mehregany, and R. L. Mullen, "Analysis of tip deflection and force of a bimetalic cantilever microactuator," Journal of Micromechanical Microelectronic Engineering, vol. 3, no. 1, pp. 4-7, 1993.

[74] Argillon, "Vibrit 1334."

[75] T. Prochazka, M. Fischer, B. Gruber, R. Manner, R. Matz, and S. Walter, "High quality ltcc resonator for voltage-controlled oscillator," IEEE Transactions on Components and Packaging Technologies, vol. 26, no. 3, pp. 591 - 597, Sept. 2003. 
[76] G. EPOXY, Produkte, "E-solder 3021."

[77] R. Pieper, A. Cooper, and G. Pelegris, "Dual baseline triangulation," vol. 26, no. 3, March 1995, pp. $424-428$.

[78] B. C. Wadell, Transmission Line Design Handbook. Artech House, 1991.

[79] H. A. Wheeler, "Transmission-line properties of parallel strips separated by dielectric sheet," IEEE Transactions on Microwave Theory and Techniques, vol. 13, no. 2, pp. 172 - 185, March 1965.

[80] R. Mongia, I. Bahl, and B. Prakash, RF and Microwave Coupled Line Circuits. Artech House, Inc., 1999.

[81] T. A. Milligan, "Dimensions of microstrip coupled lines and interdigital structures," IEEE Transactions on Microwave Theory and Techniques, vol. 25, no. 5, pp. 405 - 410, May 1977.

[82] M. Krumpholz, C. Huber, and P. Russer, "A field theoretical comparison of fdtd and tlm," IEEE Transactions on Microwave Theory and Techniques, vol. 43, no. 8, pp. 1935 - 1950, Aug. 1995.

[83] J. Brauer, B. MacNeal, L. Larkin, and V. Overbye, "New method of modeling electronic circuits coupled with 3d electromagnetic finite element models," IEEE Transactions on Magnetics, vol. 27, no. 5, pp. 4085 - 4088, Sep. 1991.

[84] M. Krumpholz and P. Russer, "A field theoretical derivation of tlm," IEEE Transactions on Microwave Theory and Techniques, vol. 42, no. 9, pp. 1660 - 1668, Sep. 1994.

[85] K. Chamberlin, K. Komisarek, and K. Sivaprasad, "A method-of-moments solution to the twisted-pair transmission line," IEEE Transactions on Electromagnetic Compatibility, vol. 37, no. 1, pp. 121 - 126, Feb. 1995.

[86] D. M. Pozar, Microwave Engineering, 2nd ed. Wiley , Inc., 1998.

[87] P. M. Inc., "Thin film electronically tunable pre-selectors for software defined radios," Microwave Journal, vol. 47, no. 10, pp. 138-144, Oct. 2004.

[88] A. Brown and G. Rebeiz, "A varactor-tuned rf filter," IEEE Transactions on Microwave Theory and Techniques, vol. 48, no. 7, pp. 1157 - 1160, July 2002.

[89] H. J. De los Santos, G. Fischer, H. A. C. Tilmans, and J. T. M. Van Beek, "Rf mems for ubiquitous wireless connectivity. part ii. applications," Microwave Magazine, vol. 5, no. 4, pp. 50-65, Dec. 2004.

[90] S. A. and, "Vibrit piezoceramics from siemens," Siemens Application Notes, 1981. 
[91] A. Morini and T. Rozzi, "On the generalized scattering matrix of a lossless multiport," in IEEE MTT-S International Microwave Symposium Digest, vol. 3, Denver, CO, June 1997, pp. $1607-1610$. 